\title{
Speckle Interferometry at the U.S. Naval Observatory. XXII.
}

\author{
Brian D. Mason and William I. Hartkopf \\ U.S. Naval Observatory \\ 3450 Massachusetts Avenue, NW, Washington, DC, 20392-5420 \\ Electronic mail: (brian.d.mason, william.hartkopf)@navy.mil
}

\begin{abstract}
The results of 4,747 intensified CCD observations of double stars, made with the 26-inch refractor of the U.S. Naval Observatory, are presented. Each observation of a system represents a combination of over two thousand short-exposure images. These observations are averaged into 2,667 mean relative positions and range in separation from 0.328 to $95^{\prime \prime} .9$, with a median separation of 8.673 . Four orbits are improved. This is the $22^{\text {nd }}$ in this series of papers and covers the period 4 January 2016 through 29 December 2016.
\end{abstract}

Subject headings: binaries : general — binaries : visual — stars : individual(BD+32 1731, HD 183063, HD 193651) - techniques : interferometric

\section{Introduction}

This is the $22^{\text {nd }}$ in a series of papers from the U.S. Naval Observatory's speckle interferometry program, presenting results of observations obtained at the USNO 26-inch telescope in Washington, DC (see, most recently, Mason et al. 2017).

From 4 January through 29 December 2016, the 26-inch telescope was used on 67 of $216(31 \%)$ scheduled nights. While most nights were lost due to weather conditions, time was also lost due to testing and upgrades of instrumentation and software, other mechanical or software issues, and to a lack of observing personnel.

Individual nightly totals varied substantially, from 7 to 200 observations per night (mean 71.3). The results yielded 4747 observations (pointings of the telescope) and 4795 resolutions (pairs measured). Not all observations result in a resolution and observations of multiple star systems in a single CCD field gives the somewhat non-intuitive result where the number of resolutions exceeds the number of observations. After removing marginal observations, calibration data, tests, and "questionable measures" a total of 4143 
measurements remained. These "questionable measures" are not all of inferior quality but may represent significant differences from the last measure, often made many decades ago. Before these measures are published they will need to be confirmed in a new observing season to account for any possible pointing or other identification problems. The tabulated list of these is retained internally and forms a "high priority observing list" for subsequent observing seasons. In this continuing process we here also present 38 measures obtained from 2002-2005. These 4181 measures were grouped into 2667 mean relative positions.

\section{Instrumentation \& Observing Technique}

In $§ 2.3$ of Mason et al. (2017) we described future planned improvements of the USNO visual double star observing system. Specifically, item \#1, which was illustrated in Figure 1 , allows us to observe closer pairs. This has several important consequences. Rather than using double stars for scale and rotation calibration, we can now return to the more traditional and fundamental technique of observing a single star through a slit-mask which produces interference fringes that can be used to determine spatial and angular calibration independently.

For all measurements produced in this program we have utilized traditional directed vector autocorrelation (hereafter DVA; Bagnuolo et al. 1992) reduction techniques, however, when observing pairs with the "backup" speckle camera we only observed wider pairs where there was no expectation that the observations were interferometric. While we called them "CCD observations" we had an expectation that the results would be better than conventional CCD astrometry due to the short-exposure time and the many thousand correlations obtained in a DVA reduction. Historically, those taken with the "backup" camera were characterized as "CCD astrometry" and those taken with the primary camera characterized as "speckle interferometry," even though there were probably speckle observations which may have been more properly CCD observations and vice-versa.

With the new camera capable of observing from the Rayleigh limit of the $26^{\prime \prime}\left(0{ }^{\prime \prime} 2\right)$ to beyond a minute of arc, all reduced with the same methodology, a new procedure to characterize the technique was needed. Speckle interferometry is most obvious when the measured separation is less than the diameter of the integrated seeing disk (e.g., McAlister et al. 1987, Figure 2). Observations of pairs with separations less than the atmospheric seeing $\left(r_{0}\right)$ would certainly be obtained by speckle interferometry. However, when observing separated pairs of similar brightness wider than the seeing disk, there can be very similar morphologies in their short-exposure magnified image structure indicative of isoplanicity, a characteristic of speckle images. Based on this and our evaluation of images taken over 
the course of the year those observations of systems with separations less than or equal to 3 3.5 are characterized as "speckle interferometry" while those greater than 3.5 are "CCD astrometry." While there is not a clear divider between these two, pairs with separations less than 3.5 usually demonstrate isoplanicity while those more than 3.5 usually do not. When the seeing monitor described in Mason et al. (2017) $§ 2.3$ comes online we can make a more rigorous characterization of observing conditions rather than this subjective divider. The pairs which we can now observe include those with shorter periods allowing us to improve the orbits of several systems, as described in $\S 3.3$ below.

\section{Results}

With the capability to observe closer pairs our observing list construction methodology has changed. The initial input list remains the Washington Double Star (hereafter, WDS, Mason et al. 2001) Catalog and the main focus of the observing list remains neglected pairs, that is, pairs not observed recently or unconfirmed. Pairs that are closer in separation tend to be both more difficult to observe and move faster, necessitating more frequent re-observation. Provided in Table 1 are our observing cadences and magnitude limits for different separation regimes. In that table, Column 1 provides the separation range, Column 2 the $\mathrm{M}_{\mathrm{v}}$ limit (of both components), Column 3 the $\Delta \mathrm{m}$ limit and Column 4 the time since the last observation for the pair to be considered neglected.

The separation range is based on the last measured value. It is likely that we could measure pairs wider than 60", especially if their position angle is along a diagonal in our field of view, but as these would likely be more slow moving not observing them as often is less impactful. While we can observe to the Rayleigh limit of 0.2 , in evaluating data for the orbit catalog (Hartkopf et al. 2001) we have found that those obtained close to the resolution limit of the telescope/filter combination are less valuable for high precision work. Consequently, we set a lower limit 50\% higher at $0^{\prime \prime} 3$. The magnitude and magnitude difference is based on the WDS values. While we strive to keep these as accurate as possible these could be incorrect. If they are, the star(s) tend to be fainter than advertised, possibly too faint to observe. These are removed from the observing list and their magnitudes updated so they should not appear on observing lists in subsequent years. Based on our experience in 2016 the magnitude limits seem appropriate while the magnitude difference limits for those under four arcseconds seem too conservative. These will be adjusted to 1.5 and 1.0, respectively, for the next observing list.

To this list of pairs which are temporally neglected $(N=4390)$ and unconfirmed $(N=59)$ additional targets are added based on other considerations: bright, and thus, 
potential objects for star-trackers $(N=254)$, having questionable observations in past years $(N=4)$, being examined for co-planarity $(N=5)$, fast-moving $(N=19)$, systems with multiple determinations in the Sixth Orbit Catalog (Hartkopf et al. 2001, $N=7$ ) or those under special analysis $(N=18)$.

On a given night a pair may be observed multiple times in different data collection modes and with different magnification as it is not always obvious which will produce the best result. Further, as object acquisition is the longest item in the duty cycle, adding additional observations is less consequential. For those intranightly observations $(n=1257)$ the rms values are quite low: $d \theta=0.08$ and $\frac{d \rho}{\rho}=0.0017$. A smaller number $(n=146)$ comprise those objects which appear to be slow moving ${ }^{1}$ and were observed on multiple nights. For those internightly observations the rms values are twice the intranightly values: $d \theta=0.15$ and $\frac{d \rho}{\rho}=0.0034$. We take these values as representative of the true error.

\subsection{New Pairs}

Table 2 presents coordinates and magnitude information from $\mathrm{CDS}^{2}$ for seven pairs which are presented here for the first time. These are closer components to known systems or pairs in the same field of view. Column one gives the coordinates of the primary of the pair. Column two is the WDS identifier while Column three is the discoverer designation (where WSI = Washington Stellar Interferometer) number. Most of these are additional components to already known pairs. For these, we retain the discovery designation of the known pair. Columns four and five give the visual magnitudes of the primary and secondary, and Column six notes the circumstance of the discovery. The mean double star positions of our $26^{\prime \prime}$ measures (T, $\theta$, and $\rho$ ) of these systems are given in Table 4.

As many of these are quite wide we are able to provide between two and seven additional measures of relative astrometry, in Table 3, from other catalogs using the same methodology as described in Wycoff et al. (2006) and Hartkopf et al. (2013). In that table, the first two columns identify the system by providing its epoch-2000 coordinates and discovery designation (as given in Table 1). Columns three through five give the epoch of observation (expressed as a fractional Julian year), the position angle (in degrees), and the separation (in seconds of arc). Note that in all tables the position angle, measured from

\footnotetext{
${ }^{1}$ We assume $\Delta \theta=\Delta \rho=0$ for these.

${ }^{2}$ magnitude information is from one of the catalogs queried in the Aladin sky atlas, operated at CDS, Strasbourg, France. See http://aladin.u-strasbg.fr/aladin.gml.
} 
North through East, has not been corrected for precession, and is thus based on the equinox for the epoch of observation. Column six and seven is the source of the measure and either a reference or note to the source.

\subsection{Measures of Known Pairs}

Tables 4 and 5 present the relative measurements of double stars made with the $26^{\prime \prime}$ telescope. Table 4 presents those with no calculation for motion, either orbital or linear. As in Table 1, the first two columns identify the system by providing its epoch-2000 coordinates and discovery designation. Columns three and four give the epoch of observation (expressed as a fractional Julian year) and the position angle (in degrees). Column five gives the position angle error. This is the internightly rms value if one is available or the mean value of 0.2 if it is not. Columns six and seven provide the separation (in seconds of arc) and its error. As above, the error is its internightly value or the mean error $(=0.0034 \rho)$. Column eight is the number of nights in the mean position. When this is " 1 " the errors in Columns five and seven are the mean results as described above. Finally, Column nine is reserved for notes.

The 2324 measures presented in Table 4 have a mean separation of $13^{\prime \prime} .897$ and a median value of $9{ }^{\prime} \cdot 055$. The mean number of years since the pair was last observed is 7.15. The seven pairs listed in Table 2 are included here, as are two objects confirmed here: $21473+4644=$ SRW 11AC, a pair first noted by Roger Sinnot (1999) and 22384+5223 $=$ BAR 61BC, a pair first measured by E.E. Barnard and whose only measure is in the catalog of Aitken \& Doolittle (1932), not measured since the discovery 101 years ago!

Table 5 presents measurements of doubles where some calculation of position (orbital or linear) is available. The first eight columns are the same as Table 4 above. Columns nine \& ten provide the $\mathrm{O}-\mathrm{C}$ residual to the determination referenced in Column eleven. The final column, like that of Table 4, provides notes. In some cases a measure has residuals to more than one calculation. In some of those cases the second calculation refers to a new orbit (Table 6) which is described below.

Not surprisingly, the objects in Table 5 are both closer and more frequently observed than those of Table 4. The 168 measures presented in Table 5 have a mean separation of $12^{\prime \prime} .777$ and a median value of 5.428 . The mean number of years since the pair was last observed is 2.59 .

In both Table $4 \& 5$ we include older measures from $2002(N=9), 2004$ (12) and 2005 (17) which were inappropriate to include in earlier entries in this measurement series. 
Two pairs, $05013+5015=$ STF 619 and $21200+5259=$ STF2789AB, each have multiple orbits in Kiselev et al. (2009). In those cases we are able to identify definitively which is the better solution. In addition to those two pairs systems are identified in the notes column whose orbital or linear solution may need to be improved in the future but where the quantity of data is insufficient to improve them at this time.

\subsection{Improved Orbits}

Eight systems with sufficient data to improve their orbits are presented in Tables 6 \& 7 and Figures $1 \& 2$. All of the individual measures were weighted by the procedures of Hartkopf et al. (2001) and calculated with the venerable "grid-search" method of Hartkopf et al. (1989).

Table 6 is broken into two groups. The first orbit we characterize as "improved but still provisional" and is given without errors. They fit the data better than the earlier orbit and should give reasonable ephemerides over the next several decades, but the elements will all require correction over the course of a complete orbit before they can be considered even approximately correct. As in earlier tables, the first two columns identify the system by providing its epoch-2000 coordinates and discovery designation. Columns three through nine provides the seven Campbell elements: the period ( $\mathrm{P}$ in years), the semimajor axis ( $\mathrm{a}^{\prime \prime}$ in arcseconds), the inclination (i) and longitude of the node $(\Omega)$, both in degrees, the epoch of the most recent periastron passage ( $\mathrm{T}_{o}$ in years), the eccentricity $(\mathrm{e})$ and the longitude of periastron ( $\omega$ in degrees). Column ten gives the reference to the previous "best" orbit and Column eleven the orbital "grade" following the procedures of Hartkopf et al. (2001).

In the second part of Table 6 are the three orbits we characterize as "reliable", all with much shorter periods than those in the first group. All eleven columns are the same as the first part of the table, however, here under each element is its formal error. The precision of the element is defined by the precision of its error. Relative visual orbits of all eight systems are plotted in Figure 1, with the $\mathrm{x}$ and y axes indicating the scale in arcseconds. Each solid curve represents the newly determined orbital elements presented in Table 6 and the dashed curve is the orbit of the earlier orbit referenced in Column ten.

Table 7 gives the ephemerides for the pairs in Table 6 over the years 2018 through 2026, in two-year increments. Columns 1 and 2 are the same identifiers as in Table 6, while Columns $3+4,5+6$, etc., through $11+12$ give predicted values of $\theta$ and $\rho$, respectively, for the years 2018.0, 2020.0, etc., through 2026.0.

Notes to individual systems follow: 


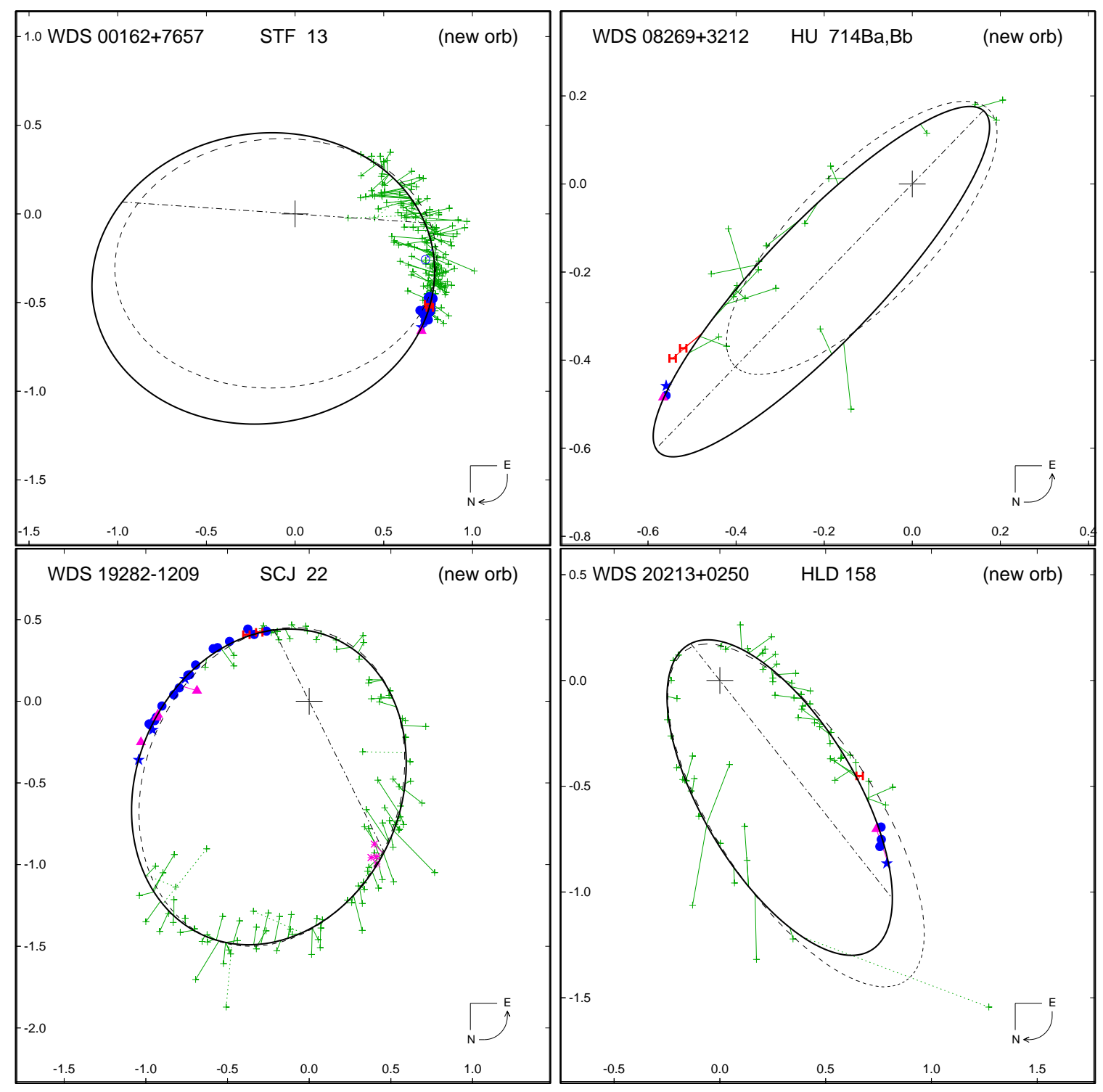

Fig. 1. - Figure 1 illustrates the new orbital solutions, plotted together with all published data in the WDS database as well as the new data in Table 5. In each of these figures, micrometric observations are indicated by plus signs, interferometric measures by filled circles, space-based measures are indicated by the letter ' $\mathrm{H}$ '. " $O-C$ " lines connect each measure to its predicted position along the new orbit (shown as a thick solid line). Dashed " $O-C$ " lines indicate measures given zero weight in the final solution. A dot-dash line indicates the line of nodes, and a curved arrow in the lower right corner of each figure indicates the direction of orbital motion. The earlier orbit referenced in Table 6 is shown as a dashed ellipse. 
$\mathbf{0 8 2 6 9}+\mathbf{3 2 1 2}=\mathbf{H U}$ 714Ba,Bb : Also known as BD +32 1731. Based on the period and semi-major axis of Table 6 and the parallax (17.30 \pm 1.77 mas; van Leeuwen 2007) the mass sum of this system is $0.775 \pm 0.409 \mathcal{M}_{\odot}$. This is not unreasonable for a $\mathrm{K} 2$ dwarf and its companion and the orbital elements and mass may see significant improvement following the pair reaching its widest separation in the apparent orbit ellipse, predicted for 2037.

19282-1209 = SCJ 22: Also known as HD 183063. Based on the period and semi-major axis of Table 6 and the parallax (27.31 \pm 0.93 mas; van Leeuwen 2007) the mass sum of this system is $1.711 \pm 0.231 \mathcal{M}_{\odot}$, which is the smallest error $(15 \%)$ of the three here. Completion of a full orbit, which is predicted for 2044, will allow the period to be very precisely determined in following years.

20213 $+\mathbf{0 2 5 0}=$ HLD 158 : Also known as HD 193651. Based on the period and semi-major axis of Table 6 and the parallax (2.27 \pm 2.28 mas; van Leeuwen 2007) the worthless mass sum of this system is $1000 \pm 3138 \mathcal{M}_{\odot}$. The small parallax and its large relative error is preventing a more precise mass determination, although like 08269+3212 above, this one has also yet to reach its largest separation in the apparent ellipse, predicted for 2067 .

\subsection{Double Stars Not Found}

Table 8 presents four systems which were observed but not detected. Possible reasons for nondetection include orbital or differential proper motion making the binary too close or too wide to resolve at the epoch of observation, a larger than expected $\Delta \mathrm{m}$, incorrect pointing of the telescope, and misprints and/or errors in the original reporting paper. It is hoped that reporting these will encourage other double star astronomers to either provide corrections to the USNO observations or to verify the lack of detection.

This research has also made use of the SIMBAD database, operated at CDS, Strasbourg, France and NASA's Astrophysics Data System. The continued instrument maintenance by the USNO instrument shop, Gary Wieder, Chris Kilian and Phillip Eakens, makes the operation of a telescope of this vintage a true delight.

\section{REFERENCES}

Aitken, R.G. \& Doolittle, E. 1932, New General Catalogue of Double Stars Within $120^{\circ}$ of the North Pole (Washington, DC: Carnegie Institution of Washington) 
Alzner, A. 1998, A\&AS 132, 253

Alzner, A. 2007, Inf. Circ. 163

Bagnuolo, W.G., Mason, B.D., Barry, D., Hartkopf, W. \& McAlister, H.A. 1992, AJ 103, 1399

Baize, P. 1978, Inf. Circ. 76

Baize, P. 1993, A\&AS 99, 205

Berko, E. 2009, JDSO 5, 168

Brendley, M. \& Hartkopf, W.I. 2007, Inf. Circ. 163

Chang, L. 1972, AJ 77, 759

Cutri, R.M., Skrutskie, M.F., van Dyk, S., et al. 2003, yCat, 2246, 0

Cutri, R.M., et al. 2012, yCat, 2311, 0

Cvetkovic, Z. \& Novakovic, B. 2006, Serbian AJ 173, 73

Cvetkovic, Z., Pavlovic, R. \& Boeva, S. 2016, AJ 151, 58

DENIS consortium 2005, yCat, 2263, 0

Docobo, J.A. \& Campo, P. 2016, Inf. Circ. 188

Docobo, J.A. \& Costa, J.M. 1984, Inf. Circ. 92

Docobo, J.A. \& Ling, J.F. 2009, AJ 138, 1159

Docobo, J.A., Tamazian, V.S., Andrade, M. \& Melikian, N.D. 2003, AJ 126, 1522

Drummond, J.D., Christou, J.C. \& Fugate, R.Q. 1995, ApJ 450, 380

Eggenberger, P., Miglio, A., Carrier, F., Fernandes, J. \& Santos, N.C. 2008, A\&A 482, 631

Friedman, E.A., Mason, B.D. \& Hartkopf, W.I. 2012, Inf. Circ. 176

Genet, R., Zirm, H., Rica, F., Richards, J., Rowe, D. \& Gray, D. 2015, JDSO 11, 200

Gorshanov, D.L., Shakht, N.A. \& Kisselev, A.A. 2006, SvA 49, 386

Grosheva, E.A. 2006, SvA 49, 397

Hale, A. 1994, AJ 107, 306

Hartkopf, W.I. \& Mason, B.D. 2011a, AJ 142, 56

Hartkopf, W.I. \& Mason, B.D. 2011b, Catalog of Rectilinear Elements, published in Second USNO Double Star CD 2006. $5^{3}$.

${ }^{3}$ See the current version at http://ad.usno.navy.mil/wds/lin1.html 
Hartkopf, W.I. \& Mason, B.D. 2011c, Inf. Circ. 175

Hartkopf, W.I. \& Mason, B.D. 2015, AJ 150, 136

Hartkopf, W.I., Mason, B.D. \& Worley, C.E. 2001, AJ 122, $3472^{4}$

Hartkopf, W.I., Mason, B.D., Finch, C.T., Zacharias, N., Wycoff, G.L. \& Hsu, D. 2013, AJ 146,76

Hartkopf, W.I., McAlister, H.A. \& Franz, O.G. 1989, AJ 98, 1014

Heintz, W.D. 1984, AJ 89, 1063

Heintz, W.D. 1996, AJ 111, 408

Heintz, W.D. 1998, ApJS 111, 335

Høg, E., Fabricius, C., Makarov, V.V., Urban, S., Corbin, T., Wycoff, G., Bastian, U., Schwekendiek, P., \& Wicenec, A. 2000, A\&A 357, 367

Hopmann, J. 1960, Mitt. Sternw. Wien 10, 155

Hopmann, J. 1964, Ann. Sternw. Wien 26, \#1

Hopmann, J. 1970, Astron. Mitt. Wien, \#5, 217

Hopmann, J. 1973, Astron. Mitt. Wien \#13, 301

Hurowitz, J.L., Hartkopf, W.I. \& Mason, B.D. 2013, Inf. Circ. 181

Hurowitz, J.L., Hartkopf, W.I. \& Mason, B.D. 2014, Inf. Circ. 182

Kiselev, A.A. \& Romanenko, L.G. 1996, Astr. Reports 40, 795

Kiselev, A.A., Romanenko, J.G. \& Kalinichenko, O.A. 2009, Astr. Reports 53, 126

Kiyaeva, O.V., Gorynya, N.A. \& Izmailov, I.S. 2010, SvAL 36, 204

Kiyaeva, O.V., Kisselev, A.A., Polyakov, E.V. \& Rafalśkii, V.B. 2001, SvAL 27, 391

Ling, J.F. 2004, Inf. Circ. 154

Mason, B.D. \& Hartkopf, W.I. 2012, Inf. Circ. 178

Mason, B.D. \& Hartkopf, W.I. 2014, Inf. Circ. 184

Mason, B.D., Douglass, G.G. \& Hartkopf, W.I. 1999, AJ 117, 1023

Mason, B.D., Hartkopf, W.I., Bredthauer, G., Ferguson, E.W., Finch, C.T., Killian, C.M., Rafferty, T.J., Ragan, T.J. \& Wieder, G.D. 2017, AJ 153, 20

\footnotetext{
${ }^{4}$ See the current version at http://ad.usno.navy.mil/wds/orb6.html.
} 
Mason, B.D., Hartkopf, W.I., Wycoff, G.L., Rafferty, T.J., Urban, S.E. \& Flagg, L. 2004, AJ 128, 3012

McAlister, H.A., Hartkopf, W.I., Hutter, D.J. \& Franz, O.G. 1987, AJ 93, 688

Mason, B.D., Wycoff, G.L., Hartkopf, W.I., Douglass, G.G. \& Worley, C.E. 2001, AJ 122, $3466^{5}$

Novakovic, B. \& Todorovic, N. 2006, Serbian AJ 172, 21

Olevic, D. \& Jovanovic, P. 2001, Serbian AJ 163, 5

Olevic, D., Popovic, G.M., Pavlovic, R. \& Cvetkovic, Z. 2003, Serbian AJ 166, 43

Olivier, C.P. 1939, Pub. Univ. Penn. 5, Pt. 2, Sec. 1

Pavlovic, R \& Todorovic, N. 2005, Serbian AJ 170, 73

Popovic, H.M. 1969, Bull. Obs. Astron. Belgrade 27, \#1, 33

Popovic, G.M. \& Pavlovic, R. 1996, Bull. Obs. Astron. Belgrade \#153, 57

Pourteau, M.A. 1933, Cat. des etoiles doubles de la zone +24deg de la carte photog. du ciel

Prieur, J.-L., Scardia, M., Pansecchi, L., Argyle, R.W. \& Sala, M. 2012, MNRAS 422, 1057

Raghavan, D., McAlister, H.A., Torres, G., Latham, D.W., Mason, B.D., Boyajian, T.S., Baines, E.K., Williams, S.J. ten Brummelaar, T.A., Farrington, C.D., Ridgway, S.T., Sturmann, L., Sturmann, J. \& Turner, N.H. 2008, ApJ 690, 394

Riddle, R.L., Tokovinin, A., Mason, B.D., Hartkopf, W.I., Roberts, L.C., Jr., Baranec, C., Law, N.M., Bui, K., Burse, M.P., Das, H.K., Dekany, R.G., Kulkarni, S., Punnadi, S., Ramaprakash, A.N. \& Tendulkar, S.P. 2015, ApJ 799, 4

Rica, F.M. 2012, JDSO 8, 127

Rica, F.M., Barrena, R., Vazquez, G., Henriquez, J.A. \& Hernandez, F. 2012, MNRAS 419, 197

Romanenko, L.G. \& Kiselev, A.A. 2014, Astron. Reports 58, 30

Scardia, M., Prieur, J.-L., Pansecchi, L. \& Argyle, R.W. 2012, Inf. Circ. 177

Scardia, M., Prieur, J.-L., Pansecchi, L., Argyle, R.W. \& Sala, M. 2011, AN 332, 508

Scardia, M., Prieur, J.-L., Pansecchi, L., Argyle, R.W. \& Zanutta, A. 2015a, Inf. Circ. 185

Scardia, M., Prieur, J.-L., Pansecchi, L., Argyle, R.W., Zanutta, A. \& Aristidi, E. 2015b, AN 336, 388

\footnotetext{
${ }^{5}$ see current version at http://ad.usno.navy.mil/wds/.
} 
Scardia, M., Prieur, J.-L., Pansecchi, L., Argyle, R.W. \& Zanutta, A. 2015c, Inf. Circ. 186

Scheiner, J. 1908, Pub. Obs. Potsdam 20, \#59

Seymour, D., Mason, B.D., Hartkopf, W.I. \& Wycoff, G.L. 2002, AJ 123, 1023

Sinnott, R.W. 1999, Sky \& Telescope 97, 100

Söderhjelm, S. 1999, A\&A 341, 121

Tokovinin, A. 2016, ApJ 831, 151

Tokovinin, A., Mason, B.D., Hartkopf, W.I., Mendez, R.A. \& Horch, E.P. 2015, AJ 150, 50

Urban, S.E., Corbin, T.E., Wycoff, G.L., Martin, J.C., Jackson, E.S., Zacharias, M.I., \& Hall, D.M. 1998, AJ 115, 1212

van Leeuwen, F. 2007, A\&A 474, 653

Wycoff, G.L., Mason, B.D. \& Urban, S.E. 2006, AJ 132, 50

Zacharias, N., Finch, C.T., Girard, T.M., Henden, A., Bartlett, J.L., Monet, D.G., \& Zacharias, M.I. 2013, AJ 145, 44

Zasche, P., Wolf, M., Hartkopf, W.I., Svoboda, P., Uhlar, R., Liakos, A. \& Gazeas, K. 2009, AJ 138, 664

Zeller, G. 1965, Ann. Sternw. Wien 26, 111

Zirm, H. 2007, Inf. Circ. 161

Zirm, H. 2008, Inf. Circ. 166

Zirm, H. 2011, JDSO 7, 24

Zirm, H. 2013, JDSO 9, 214

Zirm, H. 2014, Inf. Circ. 182

Zirm, H. 2015, Inf. Circ. 185

Zirm, H. \& Rica, F.M. 2014, Inf. Circ. 183

Zulevic, D.J. 1977, Inf. Circ. 72 
Table 1. Observing List Parameters

\begin{tabular}{rrrrr}
\hline \hline Separation Range & $\mathrm{M}_{\mathrm{v}}$ limit & $\Delta \mathrm{M}_{\mathrm{v}}$ limit & Cadence \\
\hline $60^{\prime \prime} 0>\rho \geq 10^{\prime \prime} 0$ & 12 & 3.0 & $10 \mathrm{yr}$ \\
$10^{\prime \prime} 0>\rho \geq$ & $4^{\prime \prime} 0$ & 11 & 2.0 & $5 \mathrm{yr}$ \\
$44^{\prime \prime} 0>\rho \geq$ & $1^{\prime \prime} 0$ & 10 & 1.0 & $2 \mathrm{yr}$ \\
$1^{\prime \prime} 0>\rho \geq$ & $>0$ & 0 & 0.2 & $1 \mathrm{yr}$ \\
\hline
\end{tabular}

Table 2. New Pairs

\begin{tabular}{|c|c|c|c|c|c|}
\hline \multirow{2}{*}{$\begin{array}{c}\text { Coordinates } \\
\alpha, \delta(2000)\end{array}$} & \multirow{2}{*}{$\begin{array}{c}\text { WDS } \\
\text { Designation }\end{array}$} & \multirow{2}{*}{$\begin{array}{c}\text { Discoverer } \\
\text { Designation }\end{array}$} & \multicolumn{2}{|c|}{ Magnitudes } & \multirow[t]{2}{*}{ Note } \\
\hline & & & Primary & Secondary & \\
\hline $051725.43+035313.4$ & $05175+0354$ & BAL 2142 AC & 11.67 & 13.6 & 1 \\
\hline $063152.51+045615.0$ & $06319+0457$ & SLE $293 \mathrm{GH}$ & 11.75 & 12.77 & 2 \\
\hline $071642.20+031225.0$ & $07167+0312$ & BAL 2261 AC & 11.4 & 13. & 1 \\
\hline $074418.79-004037.1$ & $07443-0040$ & BAL $825 \mathrm{BC}$ & 11.5 & 13.3 & 3 \\
\hline $081440.22+043821.8$ & $08147+0438$ & WSI 162 & 11.80 & 11.95 & \\
\hline $204113.16-131941.8$ & $20412-1320$ & WSI 163 & 10.6 & 12.5 & 4 \\
\hline $205101.51+391505.6$ & $20510+3915$ & SEI $1279 \mathrm{BC}$ & 11.9 & 12.5 & 1 \\
\hline
\end{tabular}

1: Physicality status unknown, but closer than the known pair.

2: AG \& AH both in WDS. This is a closer, smaller $\Delta \mathrm{m}$ pairing in this multiple system.

3 : This is a common proper motion pair. Based on proper motion the A component appears non-physical.

4: Magnitudes are red, not visual. 
Table 3. Data Mining Measures for New Pairs

\begin{tabular}{|c|c|c|c|c|c|c|}
\hline $\begin{array}{c}\text { WDS or } \\
\alpha, \delta(2000)\end{array}$ & $\begin{array}{c}\text { Discovery } \\
\text { Designation }\end{array}$ & JY & $\begin{array}{c}\theta \\
\left(^{\circ}\right)\end{array}$ & $\begin{array}{c}\rho \\
\left({ }^{\prime \prime}\right)\end{array}$ & Source & $\begin{array}{l}\text { Reference } \\
\text { or Note }\end{array}$ \\
\hline \multirow[t]{3}{*}{$05175+0354$} & BAL 2142 AC & 2000.07 & 289.8 & 9.10 & 2MASS & 1 \\
\hline & & 2000.100 & 289.9 & 9.086 & UCAC4 & Zacharias et al. 2013 \\
\hline & & 2010.5 & 290.4 & 9.36 & WISE & 2 \\
\hline \multirow[t]{5}{*}{$06319+0457$} & SLE $293 \mathrm{GH}$ & 1899.13 & 274.6 & 18.704 & $\mathrm{AC}$ & Urban et al. 1998 \\
\hline & & 1932.09 & 274.8 & 18.814 & $\mathrm{AC}$ & Urban et al. 1998 \\
\hline & & 1999.87 & 274.7 & 18.53 & 2MASS & 1 \\
\hline & & 2000.146 & 274.7 & 18.552 & UCAC4 & Zacharias et al. 2013 \\
\hline & & 2010.5 & 274.7 & 18.75 & WISE & 2 \\
\hline \multirow[t]{2}{*}{$07167+0312$} & BAL 2261 AC & 1999.95 & 49.3 & 4.47 & 2MASS & 1 \\
\hline & & 2000.152 & 49.1 & 4.437 & UCAC4 & Zacharias et al. 2013 \\
\hline \multirow[t]{4}{*}{$07443-0040$} & $\mathrm{BAL} \quad 825 \mathrm{BC}$ & 1998.87 & 287.3 & 17.30 & 2MASS & 1 \\
\hline & & 1999.2087 & 287.1 & 17.30 & DENIS & 3 \\
\hline & & 1999.9514 & 286.8 & 17.30 & DENIS & 3 \\
\hline & & 2000.126 & 287.2 & 17.292 & UCAC4 & Zacharias et al. 2013 \\
\hline \multirow[t]{7}{*}{$08147+0438$} & WSI 162 & 1899.15 & 248.1 & 20.018 & $\mathrm{AC}$ & Urban et al. 1998 \\
\hline & & 1910.20 & 248.4 & 20.155 & $\mathrm{AC}$ & Urban et al. 1998 \\
\hline & & 1918.17 & 248.4 & 20.180 & $\mathrm{AC}$ & Urban et al. 1998 \\
\hline & & 1991.53 & 249.9 & 20.413 & Tycho & Høg et al. 2000 \\
\hline & & 2000.07 & 249.4 & 20.54 & 2MASS & 1 \\
\hline & & 2000.183 & 249.5 & 20.594 & UCAC4 & Zacharias et al. 2013 \\
\hline & & 2010.5 & 249.5 & 20.59 & WISE & 2 \\
\hline \multirow[t]{3}{*}{$20412-1320$} & WSI 163 & 1996.6929 & 47.1 & 10.31 & DENIS & 3 \\
\hline & & 2000.37 & 46.8 & 10.259 & UCAC4 & Zacharias et al. 2013 \\
\hline & & 2000.67 & 46.6 & 10.26 & 2MASS & 1 \\
\hline \multirow[t]{2}{*}{$20510+3915$} & SEI $1279 \mathrm{BC}$ & 1998.46 & 191.0 & 7.72 & 2MASS & 1 \\
\hline & & 2002.671 & 190.8 & 7.690 & UCAC4 & Zacharias et al. 2013 \\
\hline
\end{tabular}

1 : Cutri et al. (2003), All-sky Release. See Vizier On-line Data Catalog: II/246.

2 : Cutri et al. (2012), All-Sky Release. See Vizier On-line Data Catalog: II/311.

3 : DENIS Consortium (2005), Third data release (20 Sep 2005). See Vizier On-line Data Catalog: II/263. 
Table 4. ICCD Measurements of Double Stars

\begin{tabular}{|c|c|c|c|c|c|c|c|c|c|}
\hline $\begin{array}{l}\text { WDS Desig. } \\
\alpha, \delta(2000)\end{array}$ & $\begin{array}{l}\text { Di } \\
\text { Des }\end{array}$ & $\begin{array}{l}\text { scoverer } \\
\text { signation }\end{array}$ & JY & $\begin{array}{c}\theta \\
(0)\end{array}$ & $\begin{array}{l}\sigma \theta \\
(0)\end{array}$ & $\begin{array}{c}\rho \\
\left({ }^{\prime \prime}\right)\end{array}$ & $\begin{array}{l}\sigma \rho \\
\left({ }^{\prime \prime}\right)\end{array}$ & $\mathrm{n}$ & Note \\
\hline $00012+1357$ & WNO & $12 \mathrm{AB}$ & 2016.848 & 204.3 & 0.2 & 11.692 & 0.016 & 2 & \\
\hline $00026+6606$ & STF & $3053 \mathrm{AB}$ & 2016.730 & 70.8 & 0.2 & 15.318 & 0.052 & 1 & \\
\hline $00027+5958$ & $\mathrm{ARG}$ & 47 & 2016.730 & 290.2 & 0.2 & 10.134 & 0.034 & 1 & \\
\hline $00029+4715$ & $\mathrm{~A}$ & 800 & 2016.853 & 115.1 & 0.2 & 1.626 & 0.006 & 1 & \\
\hline $00039+2759$ & HJ & 1929 AB,C & 2016.850 & 288.1 & 0.2 & 5.213 & 0.018 & 1 & \\
\hline $00043+4235$ & HJ & $1932 \mathrm{AB}$ & 2016.875 & 306.9 & 0.1 & 7.135 & 0.007 & 2 & \\
\hline $00043+4235$ & WAL & $4 \mathrm{AC}$ & 2016.875 & 188.5 & 0.2 & 46.933 & 0.160 & 1 & \\
\hline $00048+4358$ & $\mathrm{~A}$ & 203 & 2016.875 & 348.4 & 0.2 & 1.956 & 0.007 & 1 & \\
\hline 00049-1320 & GAL & 295 & 2016.891 & 20.0 & 0.2 & 39.090 & 0.133 & 1 & \\
\hline $00057+1750$ & STF & 3061 & 2016.888 & 149.2 & 0.0 & 7.820 & 0.003 & 2 & \\
\hline $00057+4239$ & A & 110 & 2016.875 & 124.2 & 0.2 & 2.093 & 0.007 & 1 & \\
\hline $00059-1426$ & HJ & 3238 & 2016.891 & 240.1 & 0.2 & 33.824 & 0.115 & 1 & \\
\hline $00059+1805$ & STF & $3060 \mathrm{AB}$ & 2016.934 & 135.8 & 0.1 & 3.407 & 0.008 & 2 & \\
\hline $00065+0155$ & HJ & 1000 & 2016.932 & 205.9 & 0.1 & 7.236 & 0.015 & 2 & \\
\hline $00066-1101$ & GAL & $3 \mathrm{AB}, \mathrm{C}$ & 2016.891 & 13.4 & 0.2 & 7.232 & 0.025 & 1 & \\
\hline $00068+5430$ & $\mathrm{ES}$ & 611 & 2016.853 & 292.3 & 0.2 & 9.918 & 0.034 & 1 & \\
\hline 00071+6309 & $\mathrm{ES}$ & $1934 \mathrm{AB}$ & 2016.730 & 69.9 & 0.2 & 7.250 & 0.025 & 1 & \\
\hline 00080-1414 & STF & 3065 & 2016.891 & 290.3 & 0.2 & 9.667 & 0.033 & 1 & \\
\hline $00087-0451$ & $\mathrm{AG}$ & 298 & 2016.913 & 12.1 & 0.0 & 15.972 & 0.007 & 2 & \\
\hline $00092+4443$ & HJ & 1001 & 2016.853 & 78.2 & 0.2 & 15.451 & 0.053 & 1 & \\
\hline $00099+0827$ & $\mathrm{STF}$ & 4 & 2016.932 & 276.5 & 0.2 & 5.243 & 0.013 & 2 & \\
\hline $00099+7329$ & WFC & 1 & 2016.730 & 36.7 & 0.2 & 6.481 & 0.022 & 1 & \\
\hline $00100+4623$ & STF & 3 & 2016.853 & 82.8 & 0.2 & 4.974 & 0.017 & 1 & \\
\hline $00116+5558$ & $\mathrm{STF}$ & 7 & 2016.785 & 210.7 & 0.2 & 1.318 & 0.004 & 1 & \\
\hline $00119+6621$ & HJL & 1 & 2016.730 & 350.7 & 0.2 & 31.030 & 0.106 & 1 & \\
\hline $00137+0635$ & $\mathrm{BU}$ & 998 & 2016.913 & 107.5 & 0.7 & 1.137 & 0.071 & 2 & \\
\hline $00137+4656$ & BRT & 63 & 2016.850 & 309.9 & 0.2 & 5.605 & 0.019 & 1 & \\
\hline $00150+0849$ & STF & 12 & 2016.848 & 147.6 & 0.1 & 11.481 & 0.020 & 2 & \\
\hline $00150+5542$ & $\mathrm{KR}$ & 3 & 2016.785 & 222.4 & 0.2 & 3.486 & 0.012 & 1 & \\
\hline $00152+7801$ & STF & 11 & 2016.785 & 193.0 & 0.2 & 8.191 & 0.028 & 1 & \\
\hline $00156+4426$ & SMA & 4 & 2016.850 & 159.4 & 0.2 & 9.539 & 0.032 & 1 & \\
\hline $00158-1159$ & STF & 14 & 2016.891 & 237.4 & 0.2 & 14.460 & 0.049 & 1 & \\
\hline
\end{tabular}


Table 4-Continued

\begin{tabular}{|c|c|c|c|c|c|c|c|c|c|}
\hline $\begin{array}{l}\text { WDS Desig. } \\
\alpha, \delta(2000)\end{array}$ & & $\begin{array}{l}\text { iscoverer } \\
\text { signation }\end{array}$ & JY & $\begin{array}{c}\theta \\
(0)\end{array}$ & $\begin{array}{l}\sigma \theta \\
(0)\end{array}$ & $\begin{array}{c}\rho \\
\left({ }^{\prime \prime}\right)\end{array}$ & $\begin{array}{l}\sigma \rho \\
\left({ }^{\prime \prime}\right)\end{array}$ & $\mathrm{n}$ & Note \\
\hline $00166+1554$ & $\mathrm{~J}$ & 1802 & 2016.918 & 126.6 & 0.2 & 4.806 & 0.016 & 1 & \\
\hline $00171-1406$ & GAL & 298 & 2016.891 & 337.4 & 0.2 & 23.078 & 0.078 & 1 & \\
\hline $00172+3508$ & ES & 312 & 2016.850 & 238.0 & 0.2 & 2.805 & 0.010 & 1 & \\
\hline $00173+3332$ & $\mathrm{ES}$ & 2210 & 2016.918 & 270.0 & 0.2 & 3.393 & 0.012 & 1 & \\
\hline $00174+0221$ & STF & 21 & 2016.932 & 52.2 & 0.1 & 7.665 & 0.008 & 2 & \\
\hline $00174+0853$ & STF & $22 \mathrm{AB}, \mathrm{C}$ & 2016.932 & 235.2 & 0.4 & 3.953 & 0.013 & 2 & \\
\hline $00174+3550$ & WEI & 1 & 2016.850 & 287.0 & 0.2 & 5.335 & 0.018 & 1 & \\
\hline $00181+5118$ & ES & 749 & 2016.853 & 146.7 & 0.2 & 9.210 & 0.031 & 1 & \\
\hline 00183-1409 & HJ & 1948 & 2016.891 & 169.1 & 0.2 & 16.542 & 0.056 & 1 & \\
\hline $00184+0344$ & HDO & $12 \mathrm{AB}$ & 2004.923 & 3.4 & 0.2 & 5.149 & 0.018 & 1 & A \\
\hline $00185+4024$ & MLB & 733 & 2016.850 & 50.5 & 0.2 & 10.341 & 0.035 & 1 & \\
\hline $00187+1559$ & STF & $25 \mathrm{AB}$ & 2016.795 & 195.7 & 0.1 & 1.049 & 0.003 & 2 & \\
\hline $00247+0013$ & $\mathrm{HDO}$ & $17 \mathrm{AC}$ & 2016.900 & 327.8 & 0.0 & 49.566 & 0.017 & 2 & \\
\hline $00270+6430$ & MLB & 277 & 2016.730 & 72.6 & 0.2 & 6.732 & 0.023 & 1 & \\
\hline $00278+5001$ & FOX & $109 \mathrm{AD}$ & 2016.850 & 270.8 & 0.2 & 22.911 & 0.078 & 1 & \\
\hline $00278+5001$ & $\mathrm{HU}$ & $507 \mathrm{AB}$ & 2016.850 & 129.1 & 0.2 & 1.668 & 0.006 & 1 & \\
\hline 00279-1914 & ARA & 516 & 2016.891 & 263.1 & 0.2 & 10.105 & 0.034 & 1 & \\
\hline $00289+4125$ & STF & 31 & 2016.779 & 56.6 & 0.2 & 5.876 & 0.020 & 1 & \\
\hline $00294+3036$ & SMA & 5 & 2016.815 & 194.2 & 0.2 & 6.907 & 0.023 & 1 & \\
\hline $00295+3137$ & $\mathrm{~J}$ & 871 & 2016.815 & 358.1 & 0.2 & 2.217 & 0.008 & 1 & \\
\hline $00300-1058$ & GAL & 301 & 2016.839 & 130.3 & 0.2 & 40.704 & 0.138 & 1 & \\
\hline $00303+5624$ & CVE & $5 \mathrm{AC}$ & 2016.875 & 153.9 & 0.0 & 16.915 & 0.000 & 2 & \\
\hline $00303+5624$ & STI & $1364 \mathrm{AB}$ & 2016.875 & 8.6 & 0.2 & 14.711 & 0.050 & 1 & \\
\hline $00310+2839$ & $\mathrm{HAU}$ & $2 \mathrm{AB}$ & 2016.839 & 279.4 & 0.2 & 12.360 & 0.042 & 1 & \\
\hline $00310+3406$ & STF & 33 & 2016.815 & 213.0 & 0.2 & 2.820 & 0.010 & 1 & \\
\hline 00316-0202 & $\mathrm{STF}$ & 35 & 2016.839 & 267.0 & 0.2 & 8.795 & 0.030 & 1 & \\
\hline $00318+0844$ & $\mathrm{~J}$ & 3280 & 2016.918 & 149.4 & 0.2 & 4.924 & 0.017 & 1 & \\
\hline $00325+5720$ & $\mathrm{~J}$ & 872 & 2016.853 & 82.3 & 0.2 & 4.169 & 0.014 & 1 & \\
\hline $00327+7807$ & STF & 34 & 2016.785 & 339.3 & 0.2 & 5.844 & 0.020 & 1 & \\
\hline $00333+3731$ & ALI & 249 & 2016.779 & 287.6 & 0.2 & 13.169 & 0.045 & 1 & \\
\hline $00337+5007$ & $\mathrm{HU}$ & 511 & 2016.875 & 177.5 & 0.2 & 4.451 & 0.015 & 1 & \\
\hline $00338+1336$ & BRT & 1166 & 2016.918 & 309.6 & 0.2 & 5.206 & 0.018 & 1 & \\
\hline
\end{tabular}


Table 4-Continued

\begin{tabular}{|c|c|c|c|c|c|c|c|c|c|}
\hline $\begin{array}{l}\text { WDS Desig. } \\
\alpha, \delta(2000)\end{array}$ & $\begin{array}{l}\text { Dis } \\
\text { Desi }\end{array}$ & $\begin{array}{l}\text { coverer } \\
\text { ignation }\end{array}$ & JY & $\begin{array}{c}\theta \\
(0)\end{array}$ & $\begin{array}{l}\sigma \theta \\
(0)\end{array}$ & $\begin{array}{c}\rho \\
\left({ }^{\prime \prime}\right)\end{array}$ & $\begin{array}{l}\sigma \rho \\
\left({ }^{\prime \prime}\right)\end{array}$ & $\mathrm{n}$ & Note \\
\hline $00352+3650$ & STF & $40 \mathrm{AB}$ & 2016.779 & 312.5 & 0.2 & 11.806 & 0.040 & 1 & \\
\hline $00352+4450$ & ROE & 62 & 2016.779 & 297.5 & 0.2 & 10.107 & 0.034 & 1 & \\
\hline $00360+2959$ & STF & $42 \mathrm{BC}$ & 2016.815 & 279.5 & 0.2 & 37.557 & 0.128 & 1 & \\
\hline $00363+1701$ & $\mathrm{~J}$ & $922 \mathrm{AC}$ & 2016.839 & 1.4 & 0.2 & 49.693 & 0.169 & 1 & \\
\hline $00369+3343$ & H 5 & $17 \mathrm{AB}$ & 2016.815 & 173.4 & 0.2 & 36.249 & 0.123 & 1 & \\
\hline 00389-1704 & ARA & 139 & 2016.818 & 123.5 & 0.2 & 15.899 & 0.054 & 1 & \\
\hline $00398-1418$ & GAL & 303 & 2016.839 & 182.7 & 0.2 & 41.370 & 0.141 & 1 & \\
\hline $00400+5549$ & ES & 936 & 2016.875 & 269.1 & 0.2 & 8.121 & 0.028 & 1 & \\
\hline $00403+4004$ & MLB & $970 \mathrm{AB}$ & 2016.918 & 347.2 & 0.2 & 4.733 & 0.016 & 1 & \\
\hline $00403+4343$ & HJ & 1044 & 2016.779 & 139.2 & 0.2 & 21.793 & 0.074 & 1 & \\
\hline $00406-1502$ & GAL & 305 & 2016.839 & 322.1 & 0.2 & 43.500 & 0.148 & 1 & \\
\hline $00412+1238$ & BRT & 1167 & 2016.029 & 89.2 & 0.2 & 4.820 & 0.016 & 1 & \\
\hline $00414+2526$ & $\mathrm{~J}$ & 1803 & 2016.918 & 99.6 & 0.2 & 2.601 & 0.009 & 1 & \\
\hline $00426+7122$ & $\mathrm{STF}$ & $48 \mathrm{AB}$ & 2016.785 & 333.7 & 0.2 & 5.482 & 0.019 & 1 & \\
\hline $00428+0453$ & BAL & 2584 & 2016.839 & 21.2 & 0.2 & 8.604 & 0.029 & 1 & \\
\hline $00429+4722$ & $\mathrm{ES}$ & 2581 & 2016.875 & 71.3 & 0.2 & 10.625 & 0.036 & 1 & \\
\hline $00430+4405$ & ES & 1408 & 2016.875 & 263.4 & 0.2 & 7.698 & 0.026 & 1 & \\
\hline $00444+3337$ & STF & 55 & 2016.815 & 330.5 & 0.2 & 2.273 & 0.008 & 1 & \\
\hline $00451+0533$ & PLQ & 8 & 2016.839 & 336.6 & 0.2 & 8.602 & 0.029 & 1 & \\
\hline $00452+5333$ & HLD & 3 & 2016.875 & 52.1 & 0.2 & 3.006 & 0.010 & 1 & \\
\hline $00454-1642$ & ARA & 141 & 2016.839 & 101.2 & 0.2 & 6.559 & 0.022 & 1 & \\
\hline $00455+4324$ & $\mathrm{BU}$ & $865 \mathrm{AB}$ & 2016.779 & 192.3 & 0.2 & 1.263 & 0.004 & 1 & \\
\hline $00470-0115$ & $\mathrm{BU}$ & 494 & 2016.839 & 159.9 & 0.2 & 1.264 & 0.004 & 1 & \\
\hline $00472+3901$ & MLB & 3 & 2016.779 & 21.4 & 0.2 & 8.087 & 0.027 & 1 & \\
\hline $00474+7239$ & STF & 57 & 2016.785 & 18.5 & 0.2 & 6.300 & 0.021 & 1 & \\
\hline $00475+4214$ & ES & 1488 & 2016.779 & 282.9 & 0.2 & 7.003 & 0.024 & 1 & \\
\hline $00480+5127$ & $\mathrm{STF}$ & $59 \mathrm{AB}$ & 2016.875 & 148.2 & 0.2 & 2.286 & 0.008 & 1 & \\
\hline $00481+2533$ & $\mathrm{HO}$ & 306 & 2016.839 & 158.9 & 0.2 & 1.464 & 0.005 & 1 & \\
\hline $00485+3924$ & MLB & $4 \mathrm{AB}$ & 2016.779 & 99.7 & 0.2 & 6.766 & 0.023 & 1 & \\
\hline $00485+3924$ & MLB & $4 \mathrm{AC}$ & 2016.779 & 174.6 & 0.2 & 13.724 & 0.047 & 1 & \\
\hline $00485+3924$ & MLB & $4 \mathrm{BC}$ & 2016.779 & 203.1 & 0.2 & 13.629 & 0.046 & 1 & \\
\hline $00486-0146$ & $\mathrm{HDO}$ & 35 & 2016.839 & 34.6 & 0.2 & 7.264 & 0.025 & 1 & \\
\hline
\end{tabular}


Table 4-Continued

\begin{tabular}{|c|c|c|c|c|c|c|c|c|c|}
\hline $\begin{array}{l}\text { WDS Desig. } \\
\alpha, \delta(2000)\end{array}$ & \multicolumn{2}{|c|}{$\begin{array}{c}\text { Discoverer } \\
\text { Designation }\end{array}$} & JY & $\begin{array}{c}\theta \\
(0)\end{array}$ & $\begin{array}{l}\sigma \theta \\
(0)\end{array}$ & $\begin{array}{c}\rho \\
\left({ }^{\prime \prime}\right)\end{array}$ & $\begin{array}{l}\sigma \rho \\
\left({ }^{\prime \prime}\right)\end{array}$ & $\mathrm{n}$ & Note \\
\hline $00493+6101$ & $\mathrm{~J}$ & 222 & 2016.853 & 181.0 & 0.2 & 2.905 & 0.010 & 1 & \\
\hline $00495+1640$ & $\mathrm{~J}$ & 924 & 2016.839 & 308.9 & 0.2 & 4.184 & 0.014 & 1 & \\
\hline $00499+2743$ & STF & 61 & 2016.839 & 116.1 & 0.2 & 4.295 & 0.015 & 1 & \\
\hline $00512+2211$ & $\mathrm{KU}$ & 70 & 2016.839 & 125.2 & 0.2 & 53.852 & 0.183 & 1 & \\
\hline $00516+3925$ & A & $1509 \mathrm{AD}$ & 2016.779 & 22.7 & 0.2 & 18.570 & 0.063 & 1 & \\
\hline $00516+6859$ & $\mathrm{BU}$ & 781 & 2016.853 & 22.8 & 0.2 & 1.034 & 0.004 & 1 & \\
\hline $00527+6852$ & STF & 65 & 2016.785 & 220.1 & 0.2 & 3.198 & 0.011 & 1 & \\
\hline $00528+2714$ & BVD & 14 & 2016.839 & 225.0 & 0.2 & 12.824 & 0.044 & 1 & \\
\hline $00528+5638$ & $\mathrm{BU}$ & $1 \mathrm{AB}$ & 2016.875 & 83.2 & 0.2 & 1.522 & 0.005 & 1 & \\
\hline $00528+5638$ & $\mathrm{BU}$ & $1 \mathrm{AC}$ & 2016.875 & 134.6 & 0.2 & 3.898 & 0.013 & 1 & \\
\hline $00528+5638$ & $\mathrm{BU}$ & $1 \mathrm{AD}$ & 2016.875 & 194.8 & 0.2 & 8.973 & 0.031 & 1 & \\
\hline $00528+5638$ & $\mathrm{BU}$ & $1 \mathrm{CD}$ & 2016.875 & 220.4 & 0.2 & 7.776 & 0.026 & 1 & \\
\hline $00533+0500$ & STF & 71 & 2016.839 & 339.4 & 0.2 & 8.882 & 0.030 & 1 & \\
\hline $00534+1159$ & HJ & 9 & 2016.839 & 100.7 & 0.2 & 12.499 & 0.042 & 1 & \\
\hline $00535+0318$ & $\mathrm{HDO}$ & 37 & 2016.849 & 230.5 & 0.4 & 1.649 & 0.004 & 2 & \\
\hline $00547+0834$ & WZ & 2 & 2016.776 & 269.2 & 0.2 & 8.600 & 0.006 & 2 & \\
\hline $00550+0834$ & $\mathrm{~J}$ & 638 & 2016.728 & 207.1 & 0.2 & 3.301 & 0.011 & 1 & \\
\hline $00551+2811$ & A & 437 & 2016.883 & 29.8 & 0.2 & 3.021 & 0.010 & 1 & \\
\hline $00551+6422$ & $\mathrm{ES}$ & 118 & 2016.853 & 64.5 & 0.2 & 2.456 & 0.008 & 1 & \\
\hline $00557+5748$ & $\mathrm{ES}$ & 405 & 2016.880 & 117.2 & 0.2 & 4.391 & 0.015 & 1 & \\
\hline $00574+2053$ & $\mathrm{~J}$ & 2587 & 2016.971 & 113.7 & 0.2 & 4.047 & 0.014 & 1 & \\
\hline $00580+0917$ & WNC & 1 & 2016.969 & 132.1 & 0.3 & 5.314 & 0.014 & 2 & \\
\hline $00580+4954$ & ES & 1053 & 2016.880 & 83.1 & 0.2 & 5.523 & 0.019 & 1 & \\
\hline $00581+2655$ & STF & 77 & 2016.883 & 119.7 & 0.2 & 10.282 & 0.035 & 1 & \\
\hline $00582-1541$ & S & 390 & 2016.818 & 216.3 & 0.2 & 6.473 & 0.022 & 1 & \\
\hline $00586+4709$ & BRT & 73 & 2016.880 & 70.2 & 0.2 & 4.730 & 0.016 & 1 & \\
\hline $00588+2441$ & $A G$ & 10 & 2016.818 & 112.6 & 0.2 & 4.431 & 0.015 & 1 & \\
\hline $00588+4455$ & HJ & 1060 & 2016.880 & 288.6 & 0.2 & 8.811 & 0.030 & 1 & \\
\hline $00591+0523$ & STF & 78 & 2016.964 & 245.0 & 0.3 & 5.038 & 0.005 & 2 & \\
\hline $00594+3746$ & ES & 155 & 2016.883 & 74.0 & 0.2 & 6.406 & 0.022 & 1 & \\
\hline $01001+4443$ & STF & 79 & 2016.880 & 194.5 & 0.2 & 7.885 & 0.027 & 1 & \\
\hline $01003+3057$ & HJ & 630 & 2016.883 & 197.9 & 0.2 & 6.270 & 0.021 & 1 & \\
\hline
\end{tabular}


Table 4-Continued

\begin{tabular}{|c|c|c|c|c|c|c|c|c|c|}
\hline $\begin{array}{l}\text { WDS Desig. } \\
\alpha, \delta(2000)\end{array}$ & $\begin{array}{l}\text { Dis } \\
\text { Desi }\end{array}$ & $\begin{array}{l}\text { coverer } \\
\text { ignation }\end{array}$ & JY & $\begin{array}{l}\theta \\
(0)\end{array}$ & $\begin{array}{l}\sigma \theta \\
(0)\end{array}$ & $\begin{array}{c}\rho \\
\left({ }^{\prime \prime}\right)\end{array}$ & $\begin{array}{l}\sigma \rho \\
\left({ }^{\prime \prime}\right)\end{array}$ & $\mathrm{n}$ & Note \\
\hline $01004+1803$ & BRT & 1927 & 2016.922 & 171.8 & 0.4 & 1.955 & 0.018 & 2 & \\
\hline $01004+3228$ & ES & 317 & 2016.883 & 194.4 & 0.2 & 7.025 & 0.024 & 1 & \\
\hline $01005-1705$ & $\mathrm{BU}$ & $234 \mathrm{AB}$ & 2016.818 & 333.1 & 0.2 & 4.748 & 0.016 & 1 & \\
\hline $01005-1705$ & $\mathrm{BU}$ & $234 \mathrm{AC}$ & 2016.818 & 128.9 & 0.2 & 62.679 & 0.213 & 1 & \\
\hline $01007+0929$ & STF & $82 \mathrm{AB}$ & 2016.922 & 304.7 & 0.0 & 1.845 & 0.007 & 2 & \\
\hline $01009-1857$ & $\mathrm{CBL}$ & 116 & 2016.818 & 220.7 & 0.2 & 25.462 & 0.087 & 1 & \\
\hline $01019+2347$ & $\mathrm{AG}$ & 12 & 2016.730 & 237.9 & 0.2 & 5.856 & 0.020 & 1 & \\
\hline $01026+6135$ & ES & 1869 & 2016.853 & 144.0 & 0.2 & 6.166 & 0.021 & 1 & \\
\hline $01027+4742$ & HJ & 2010 & 2016.880 & 271.3 & 0.2 & 9.934 & 0.034 & 1 & \\
\hline $01052+2614$ & HJ & 1067 & 2016.883 & 239.8 & 0.2 & 16.043 & 0.055 & 1 & \\
\hline $01055+1523$ & STF & 87 & 2016.964 & 204.0 & 0.4 & 6.078 & 0.037 & 2 & \\
\hline $01057+2128$ & $\mathrm{STF}$ & $88 \mathrm{AB}$ & 2016.850 & 159.4 & 0.2 & 29.797 & 0.031 & 2 & \\
\hline $01058+0455$ & STF & $90 \mathrm{AB}$ & 2016.776 & 83.8 & 0.2 & 33.112 & 0.059 & 2 & \\
\hline $01062+2509$ & $\mathrm{COU}$ & 77 & 2016.883 & 231.6 & 0.2 & 2.830 & 0.010 & 1 & \\
\hline $01092+4024$ & ALI & 1014 & 2016.918 & 338.6 & 0.2 & 12.759 & 0.043 & 1 & \\
\hline $01099+4011$ & $\mathrm{AG}$ & 15 & 2016.883 & 70.9 & 0.2 & 2.828 & 0.010 & 1 & \\
\hline $01102+2447$ & POU & 107 & 2016.818 & 13.4 & 0.2 & 7.724 & 0.026 & 1 & \\
\hline $01103+1636$ & STF & $94 \mathrm{AC}$ & 2016.850 & 281.6 & 0.1 & 20.484 & 0.022 & 2 & \\
\hline $01107+8021$ & STF & 89 & 2016.785 & 320.4 & 0.2 & 16.936 & 0.058 & 1 & \\
\hline $01119+4748$ & $\mathrm{BU}$ & 398 & 2016.880 & 44.4 & 0.2 & 1.832 & 0.006 & 1 & \\
\hline 01121-1338 & SKI & 1 & 2016.728 & 250.8 & 0.2 & 8.282 & 0.028 & 1 & \\
\hline $01121+4700$ & $\mathrm{BU}$ & 236 & 2016.880 & 114.5 & 0.2 & 5.315 & 0.018 & 1 & \\
\hline $01137+0735$ & STF & $100 \mathrm{AB}$ & 2016.849 & 63.3 & 0.1 & 22.883 & 0.045 & 2 & \\
\hline $01144-0755$ & STFA & $3 \mathrm{AB}$ & 2016.731 & 330.9 & 0.2 & 49.247 & 0.115 & 2 & \\
\hline $01146+4102$ & $\mathrm{AG}$ & 300 & 2016.883 & 45.4 & 0.2 & 6.605 & 0.022 & 1 & \\
\hline $01152-1318$ & GAL & 36 & 2016.818 & 265.1 & 0.2 & 12.535 & 0.043 & 1 & \\
\hline $01162+5004$ & $\mathrm{HU}$ & 1026 & 2016.880 & 224.9 & 0.2 & 4.086 & 0.014 & 1 & \\
\hline 01163-0709 & STF & 106 & 2016.849 & 307.2 & 0.1 & 4.636 & 0.010 & 2 & \\
\hline $01172+6810$ & HJ & 1075 & 2016.853 & 104.9 & 0.2 & 7.802 & 0.027 & 1 & \\
\hline $01178-1220$ & STF & 110 & 2016.010 & 353.8 & 0.2 & 7.449 & 0.025 & 1 & \\
\hline $01186+1316$ & $\mathrm{~J}$ & 2715 & 2016.922 & 75.2 & 0.2 & 5.529 & 0.047 & 2 & \\
\hline $01188+4248$ & $\mathrm{ROE}$ & 63 & 2016.883 & 98.8 & 0.2 & 8.423 & 0.029 & 1 & \\
\hline
\end{tabular}


Table 4-Continued

\begin{tabular}{|c|c|c|c|c|c|c|c|c|c|}
\hline \multirow{2}{*}{$\begin{array}{c}\text { WDS Desig. } \\
\alpha, \delta(2000) \\
01189+6610\end{array}$} & \multicolumn{2}{|c|}{$\begin{array}{l}\text { Discoverer } \\
\text { Designation }\end{array}$} & \multirow{2}{*}{$\begin{array}{c}\text { JY } \\
2016.853\end{array}$} & \multirow{2}{*}{$\begin{array}{c}\theta \\
(\circ) \\
184.9\end{array}$} & \multirow{2}{*}{$\begin{array}{l}\sigma \theta \\
(\mathrm{o}) \\
0.2\end{array}$} & \multirow{2}{*}{$\begin{array}{c}\rho \\
\left({ }^{\prime \prime}\right) \\
2.976\end{array}$} & \multirow{2}{*}{$\begin{array}{c}\sigma \rho \\
\left({ }^{\prime \prime}\right) \\
0.010\end{array}$} & \multirow{2}{*}{$\begin{array}{l}\mathrm{n} \\
\mathrm{P} \\
1\end{array}$} & \multirow{2}{*}{$\begin{array}{l}\text { Note } \\
\end{array}$} \\
\hline & STF & $105 \mathrm{AB}$ & & & & & & & \\
\hline $01197+6135$ & KR & 11 & 2016.853 & 59.8 & 0.2 & 1.951 & 0.007 & 1 & \\
\hline $01198+5029$ & ES & 449 & 2016.880 & 215.5 & 0.2 & 11.593 & 0.039 & 1 & \\
\hline $01199+0350$ & CHE & 29 & 2016.964 & 211.0 & 0.2 & 4.264 & 0.008 & 2 & \\
\hline $01201+3639$ & WEI & $3 \mathrm{AB}$ & 2016.779 & 187.4 & 0.2 & 4.868 & 0.017 & 1 & \\
\hline $01204+0937$ & GWP & $171 \mathrm{AB}$ & 2016.818 & 213.5 & 0.2 & 46.633 & 0.159 & 1 & \\
\hline $01204+5652$ & ES & 1809 & 2016.850 & 119.8 & 0.2 & 6.044 & 0.021 & 1 & \\
\hline $01207+4620$ & STF & $112 \mathrm{BC}$ & 2016.850 & 189.3 & 0.2 & 4.665 & 0.016 & 1 & \\
\hline 01223-0019 & BAL & 648 & 2016.818 & 258.8 & 0.2 & 9.389 & 0.032 & 1 & \\
\hline $01241-1244$ & GAL & 308 & 2016.826 & 16.7 & 0.2 & 24.863 & 0.085 & 1 & \\
\hline $01245-1615$ & $\mathrm{HU}$ & 418 & 2016.826 & 102.0 & 0.2 & 4.135 & 0.014 & 1 & \\
\hline $01251+0304$ & $\mathrm{HDO}$ & $50 \mathrm{~A}, \mathrm{BC}$ & 2016.818 & 261.4 & 0.2 & 33.945 & 0.115 & 1 & \\
\hline $01255+2832$ & $\mathrm{HO}$ & 310 & 2016.779 & 356.6 & 0.2 & 1.720 & 0.006 & 1 & \\
\hline $01263-1354$ & STF & 124 & 2016.010 & 233.9 & 0.2 & 7.182 & 0.024 & 1 & \\
\hline $01282-0817$ & BRT & 351 & 2016.010 & 302.7 & 0.2 & 3.909 & 0.013 & 1 & \\
\hline $01282+3441$ & $\mathrm{HO}$ & $8 \mathrm{AB}$ & 2016.779 & 252.8 & 0.2 & 4.334 & 0.015 & 1 & \\
\hline $01286+1440$ & ARN & 88 & 2016.818 & 253.7 & 0.2 & 23.750 & 0.081 & 1 & \\
\hline $01296+3025$ & $\mathrm{ROE}$ & 105 & 2016.779 & 0.1 & 0.2 & 5.266 & 0.018 & 1 & \\
\hline $01301+4131$ & HJ & 1081 & 2016.875 & 319.2 & 0.2 & 8.513 & 0.029 & 1 & \\
\hline $01303+1239$ & STF & 129 & 2016.818 & 282.6 & 0.2 & 8.907 & 0.030 & 1 & \\
\hline $01311+4337$ & $\mathrm{ES}$ & 4 & 2016.875 & 100.7 & 0.2 & 2.766 & 0.009 & 1 & \\
\hline $01320+1150$ & OCC & $391 \mathrm{AB}, \mathrm{C}$ & 2016.818 & 122.6 & 0.2 & 7.872 & 0.027 & 1 & \\
\hline $01320-1604$ & GAL & 310 & 2016.826 & 302.4 & 0.2 & 16.166 & 0.055 & 1 & \\
\hline $01321+1657$ & STF & $132 \mathrm{DF}$ & 2016.818 & 290.1 & 0.2 & 5.285 & 0.018 & 1 & \\
\hline $01325+8523$ & TDS & 52 & 2016.785 & 294.5 & 0.2 & 1.457 & 0.005 & 1 & \\
\hline $01327-0139$ & FIL & 2 & 2016.010 & 339.7 & 0.2 & 30.567 & 0.104 & 1 & \\
\hline $01331+5416$ & ES & 2585 & 2016.850 & 30.1 & 0.2 & 15.098 & 0.051 & 1 & \\
\hline $01335-0331$ & HJ & 640 & 2016.826 & 112.2 & 0.2 & 5.325 & 0.018 & 1 & \\
\hline $01340+4559$ & ARG & 5 & 2016.850 & 320.0 & 0.2 & 9.951 & 0.034 & 1 & \\
\hline $01344+5553$ & HJ & 2047 & 2016.850 & 57.4 & 0.2 & 13.329 & 0.045 & 1 & \\
\hline $01354+4123$ & ES & 1494 & 2016.875 & 7.7 & 0.2 & 5.627 & 0.019 & 1 & \\
\hline $01355+3118$ & STF & 137 & 2016.779 & 85.5 & 0.2 & 3.409 & 0.012 & 1 & \\
\hline
\end{tabular}


Table 4-Continued

\begin{tabular}{|c|c|c|c|c|c|c|c|c|c|}
\hline $\begin{array}{l}\text { WDS Desig. } \\
\alpha, \delta(2000)\end{array}$ & $\begin{array}{l}\text { Dis } \\
\text { Des }\end{array}$ & $\begin{array}{l}\text { scoverer } \\
\text { ignation }\end{array}$ & JY & $\begin{array}{c}\theta \\
(0)\end{array}$ & $\begin{array}{l}\sigma \theta \\
(0)\end{array}$ & $\begin{array}{c}\rho \\
\left({ }^{\prime \prime}\right)\end{array}$ & $\begin{array}{l}\sigma \rho \\
\left({ }^{\prime \prime}\right)\end{array}$ & $\mathrm{n}$ & Note \\
\hline $01368+6431$ & MLB & 187 & 2016.850 & 257.0 & 0.2 & 5.593 & 0.019 & 1 & \\
\hline $01376+0709$ & PLQ & 19 & 2016.818 & 76.5 & 0.2 & 40.275 & 0.137 & 1 & \\
\hline $01379+2554$ & A & 2007 & 2016.779 & 220.4 & 0.2 & 4.641 & 0.016 & 1 & \\
\hline $01389+7643$ & $\mathrm{HU}$ & 1030 & 2016.785 & 320.6 & 0.2 & 0.795 & 0.003 & 1 & \\
\hline $01391+1211$ & HJ & 17 & 2016.818 & 281.3 & 0.2 & 8.233 & 0.028 & 1 & \\
\hline $01396-0231$ & HJ & 641 & 2016.826 & 123.5 & 0.2 & 5.799 & 0.020 & 1 & \\
\hline $01396-1825$ & $\mathrm{UC}$ & 598 & 2016.826 & 348.7 & 0.2 & 15.122 & 0.051 & 1 & \\
\hline $01397+4602$ & EGB & 1 & 2016.850 & 147.0 & 0.2 & 5.750 & 0.020 & 1 & \\
\hline $01401+3858$ & STF & 141 & 2016.779 & 303.7 & 0.2 & 1.705 & 0.006 & 1 & \\
\hline 01404-0003 & STF & 144 & 2016.029 & 293.7 & 0.2 & 15.663 & 0.053 & 1 & \\
\hline $01409+4952$ & $\mathrm{HU}$ & $531 \mathrm{BC}$ & 2016.850 & 281.0 & 0.2 & 6.189 & 0.021 & 1 & \\
\hline $01413+4435$ & BJN & 9002 & 2016.850 & 114.6 & 0.2 & 17.652 & 0.060 & 1 & \\
\hline $01419+3022$ & MLB & 679 & 2016.918 & 238.3 & 0.2 & 6.612 & 0.022 & 1 & \\
\hline $01420+5547$ & HJ & 2066 & 2016.850 & 72.6 & 0.2 & 20.816 & 0.071 & 1 & \\
\hline $01425+4813$ & BJN & 27 & 2016.850 & 47.5 & 0.2 & 4.134 & 0.014 & 1 & \\
\hline $01428+4348$ & BJN & 1 & 2016.875 & 31.2 & 0.2 & 7.792 & 0.026 & 1 & \\
\hline $01430+4207$ & BJN & 8 & 2016.875 & 304.0 & 0.2 & 13.743 & 0.047 & 1 & \\
\hline $01430+6402$ & $\mathrm{PKO}$ & $24 \mathrm{AC}$ & 2016.785 & 62.3 & 0.2 & 34.626 & 0.118 & 1 & \\
\hline $01430+6402$ & PKO & $24 \mathrm{BC}$ & 2016.785 & 77.2 & 0.2 & 32.732 & 0.111 & 1 & \\
\hline $01430+6402$ & STI & $264 \mathrm{AB}$ & 2016.785 & 352.0 & 0.2 & 8.965 & 0.030 & 1 & \\
\hline $01431+3917$ & MLB & 8 & 2016.875 & 100.2 & 0.2 & 6.509 & 0.022 & 1 & \\
\hline $01431+4238$ & ROE & 65 & 2016.875 & 122.6 & 0.2 & 9.021 & 0.031 & 1 & \\
\hline $01433-1736$ & HJ & 3455 & 2016.826 & 73.9 & 0.2 & 23.785 & 0.081 & 1 & \\
\hline $01435-1038$ & GAL & 313 & 2016.826 & 193.1 & 0.2 & 33.531 & 0.114 & 1 & \\
\hline $01443+6652$ & $\mathrm{HAU}$ & 10 & 2016.785 & 100.9 & 0.2 & 9.217 & 0.031 & 1 & \\
\hline $01450+4342$ & STF & 154 & 2016.850 & 127.4 & 0.2 & 5.224 & 0.018 & 1 & \\
\hline $01451-2003$ & HJ & 3459 & 2016.891 & 90.1 & 0.2 & 19.689 & 0.067 & 1 & \\
\hline 01456-1409 & HJ & 2081 & 2016.826 & 145.9 & 0.2 & 11.583 & 0.039 & 1 & \\
\hline $01459+1500$ & $A G$ & 23 & 2016.891 & 46.8 & 0.2 & 29.500 & 0.100 & 1 & \\
\hline $01460+6113$ & STF & 151 & 2016.853 & 38.8 & 0.2 & 7.172 & 0.024 & 1 & \\
\hline $01479+4440$ & A & 949 & 2016.932 & 294.6 & 0.1 & 3.543 & 0.025 & 2 & \\
\hline $01490-0036$ & $\mathrm{HDO}$ & 53 & 2016.891 & 26.3 & 0.2 & 7.962 & 0.027 & 1 & \\
\hline
\end{tabular}


Table 4-Continued

\begin{tabular}{|c|c|c|c|c|c|c|c|c|c|}
\hline \multirow{2}{*}{$\begin{array}{c}\text { WDS Desig. } \\
\alpha, \delta(2000) \\
01492+3404\end{array}$} & \multicolumn{2}{|c|}{$\begin{array}{l}\text { Discoverer } \\
\text { Designation }\end{array}$} & JY & \multirow{2}{*}{$\begin{array}{c}\theta \\
(0) \\
96.2\end{array}$} & \multirow{2}{*}{$\begin{array}{l}\sigma \theta \\
(\mathrm{o}) \\
0.2\end{array}$} & \multirow{2}{*}{$\begin{array}{c}\rho \\
\left({ }^{\prime \prime}\right) \\
9.859\end{array}$} & \multirow{2}{*}{$\begin{array}{c}\sigma \rho \\
\left({ }^{\prime \prime}\right) \\
0.034\end{array}$} & \multirow{2}{*}{$\begin{array}{l}\mathrm{n} \\
\mathrm{P} \\
1\end{array}$} & \multirow{2}{*}{$\begin{array}{l}\text { Note } \\
\end{array}$} \\
\hline & STF & 164 & 2016.883 & & & & & & \\
\hline $01493+4058$ & ES & 158 & 2016.968 & 48.0 & 0.0 & 7.434 & 0.024 & 2 & \\
\hline $01493+4754$ & STF & $162 \mathrm{AC}$ & 2016.932 & 178.9 & 0.1 & 20.767 & 0.021 & 2 & \\
\hline $01493+4754$ & STF & $162 \mathrm{BC}$ & 2016.932 & 177.0 & 0.0 & 19.051 & 0.004 & 2 & \\
\hline $01495+2842$ & STF & 176 & 2016.891 & 331.8 & 0.2 & 23.825 & 0.081 & 1 & \\
\hline $01495+5645$ & HJ & $2082 \mathrm{AB}, \mathrm{C}$ & 2016.880 & 126.5 & 0.2 & 16.090 & 0.055 & 1 & \\
\hline $01496+5248$ & HJ & 2085 & 2016.875 & 60.1 & 0.2 & 9.467 & 0.032 & 1 & \\
\hline $01501+2217$ & STF & 174 & 2016.891 & 164.7 & 0.2 & 2.893 & 0.010 & 1 & \\
\hline $01501+4226$ & BJN & 2 & 2016.920 & 328.3 & 0.0 & 8.915 & 0.014 & 2 & \\
\hline $01513+6451$ & STF & $163 \mathrm{AB}$ & 2016.853 & 37.9 & 0.2 & 34.525 & 0.117 & 1 & \\
\hline $01516+5252$ & $\mathrm{BU}$ & $1366 \mathrm{AC}$ & 2016.897 & 337.0 & 0.0 & 50.829 & 0.031 & 2 & \\
\hline $01519+5838$ & HJ & 1093 & 2016.880 & 24.0 & 0.2 & 4.918 & 0.017 & 1 & \\
\hline $01520+0516$ & BAL & 2597 & 2016.891 & 150.9 & 0.2 & 9.420 & 0.032 & 1 & \\
\hline $01520+1049$ & STF & 178 & 2016.891 & 206.1 & 0.2 & 3.042 & 0.010 & 1 & \\
\hline 01523-0944 & $\mathrm{BU}$ & 259 & 2016.891 & 239.4 & 0.2 & 4.849 & 0.016 & 1 & \\
\hline $01524+4125$ & MLB & 9 & 2016.893 & 320.3 & 0.0 & 6.207 & 0.020 & 2 & \\
\hline $01526+3127$ & HJ & 645 & 2016.883 & 105.6 & 0.2 & 7.726 & 0.026 & 1 & \\
\hline $01527+5717$ & ARG & $6 \mathrm{AB}$ & 2016.880 & 136.6 & 0.2 & 14.927 & 0.051 & 1 & \\
\hline $01527+5717$ & WAL & $14 \mathrm{AC}$ & 2016.880 & 103.4 & 0.2 & 66.504 & 0.226 & 1 & \\
\hline $01532+3719$ & STF & 179 & 2016.883 & 161.0 & 0.2 & 3.518 & 0.012 & 1 & \\
\hline $01534+7003$ & STF & 169 & 2016.853 & 137.2 & 0.2 & 5.332 & 0.018 & 1 & \\
\hline $01535+1918$ & STF & $180 \mathrm{AB}$ & 2016.891 & 1.3 & 0.2 & 7.387 & 0.025 & 1 & \\
\hline $01537+1822$ & $\mathrm{~J}$ & 2718 & 2016.029 & 48.2 & 0.2 & 4.770 & 0.016 & 1 & \\
\hline $01551+2847$ & STF & $183 \mathrm{AB}, \mathrm{C}$ & 2016.891 & 162.1 & 0.2 & 5.592 & 0.019 & 1 & \\
\hline $01553+4647$ & BJN & 4 & 2016.932 & 169.4 & 0.1 & 7.219 & 0.008 & 2 & \\
\hline $01554+7613$ & STF & 170 & 2016.853 & 243.5 & 0.2 & 3.170 & 0.011 & 1 & \\
\hline $01556+2732$ & MLB & 635 & 2016.891 & 164.9 & 0.2 & 6.986 & 0.024 & 1 & \\
\hline $01561+6035$ & $\mathrm{AG}$ & 301 & 2016.853 & 262.9 & 0.2 & 8.636 & 0.029 & 1 & \\
\hline $01562+3602$ & $\mathrm{AG}$ & 25 & 2016.883 & 71.0 & 0.2 & 5.473 & 0.019 & 1 & \\
\hline $01563+3758$ & ARN & 87 & 2016.883 & 284.8 & 0.2 & 56.350 & 0.192 & 1 & \\
\hline $01564+6116$ & $\mathrm{STF}$ & $182 \mathrm{AB}$ & 2016.853 & 125.1 & 0.2 & 3.623 & 0.012 & 1 & \\
\hline $01567+3505$ & ES & 2144 & 2016.883 & 143.8 & 0.2 & 6.342 & 0.022 & 1 & \\
\hline
\end{tabular}


Table 4-Continued

\begin{tabular}{|c|c|c|c|c|c|c|c|c|c|}
\hline $\begin{array}{l}\text { WDS Desig. } \\
\alpha, \delta(2000)\end{array}$ & $\begin{array}{l}\text { Dis } \\
\text { Desi }\end{array}$ & $\begin{array}{l}\text { coverer } \\
\text { ignation }\end{array}$ & JY & $\begin{array}{c}\theta \\
(0)\end{array}$ & $\begin{array}{l}\sigma \theta \\
(0)\end{array}$ & $\begin{array}{c}\rho \\
\left({ }^{\prime \prime}\right)\end{array}$ & $\begin{array}{l}\sigma \rho \\
\left({ }^{\prime \prime}\right)\end{array}$ & $\mathrm{n}$ & Note \\
\hline $01577+3646$ & ES & 162 & 2016.883 & 209.0 & 0.2 & 12.111 & 0.041 & 1 & \\
\hline $01579+2336$ & H 5 & $12 \mathrm{AB}$ & 2016.891 & 48.1 & 0.2 & 37.352 & 0.127 & 1 & \\
\hline $01586+5545$ & $\mathrm{ES}$ & $949 \mathrm{AB}$ & 2016.875 & 264.7 & 0.2 & 5.712 & 0.019 & 1 & \\
\hline $01586+5545$ & $\mathrm{ES}$ & $949 \mathrm{AC}$ & 2016.875 & 20.3 & 0.2 & 26.099 & 0.089 & 1 & \\
\hline $01588+5517$ & SMA & 25 & 2016.875 & 103.8 & 0.2 & 26.468 & 0.090 & 1 & \\
\hline $01592+0330$ & LDS & 3331 & 2016.010 & 53.7 & 0.2 & 3.934 & 0.013 & 1 & \\
\hline $01594+5036$ & $\mathrm{AG}$ & 302 & 2016.990 & 3.5 & 0.2 & 14.708 & 0.050 & 1 & \\
\hline $01596+2100$ & STF & $196 \mathrm{BC}$ & 2016.891 & 154.0 & 0.2 & 20.935 & 0.071 & 1 & \\
\hline $01596+5036$ & ES & 454 & 2016.967 & 212.1 & 0.0 & 8.366 & 0.000 & 2 & \\
\hline $01596+5623$ & HJ & $2101 \mathrm{AB}$ & 2016.880 & 278.4 & 0.2 & 5.787 & 0.020 & 1 & \\
\hline $02002+4427$ & STF & 195 & 2016.962 & 195.6 & 0.3 & 3.034 & 0.007 & 2 & \\
\hline 02003-0138 & BAL & 9 & 2016.891 & 337.5 & 0.2 & 5.668 & 0.019 & 1 & \\
\hline $02009+5258$ & A & 1921 & 2016.932 & 68.5 & 0.1 & 2.903 & 0.026 & 2 & \\
\hline $02036+7949$ & MLR & 446 & 2016.853 & 37.3 & 0.2 & 1.995 & 0.007 & 1 & \\
\hline $02042+5257$ & HJ & 2104 & 2016.932 & 170.6 & 0.0 & 30.319 & 0.000 & 2 & \\
\hline $02045+4750$ & WFC & 7 & 2016.948 & 121.0 & 0.0 & 5.299 & 0.009 & 2 & \\
\hline $02048+5701$ & $\mathrm{KR}$ & 13 & 2016.880 & 345.9 & 0.2 & 3.989 & 0.014 & 1 & \\
\hline $02051+5823$ & TOB & 13 & 2016.919 & 5.2 & 0.2 & 20.824 & 0.071 & 1 & \\
\hline $02054-0947$ & $\mathrm{~J}$ & 1450 & 2016.891 & 2.6 & 0.2 & 6.146 & 0.021 & 1 & \\
\hline $02057+5448$ & STI & 1772 & 2016.932 & 163.1 & 0.2 & 13.728 & 0.045 & 2 & \\
\hline $02057+5457$ & SMA & 28 & 2016.932 & 85.5 & 0.1 & 15.115 & 0.009 & 2 & \\
\hline $02073+5245$ & $\mathrm{UC}$ & 27 & 2016.880 & 237.8 & 0.2 & 14.507 & 0.049 & 1 & \\
\hline $02073+5507$ & HJ & 2109 & 2016.037 & 217.1 & 0.2 & 8.970 & 0.030 & 1 & \\
\hline $02074+2810$ & $\mathrm{CHE}$ & 51 & 2016.900 & 255.3 & 0.0 & 9.568 & 0.025 & 2 & \\
\hline $02076+1535$ & STF & 214 & 2016.904 & 189.8 & 0.2 & 5.248 & 0.010 & 2 & \\
\hline $02078+5525$ & SMA & 30 & 2016.037 & 328.0 & 0.2 & 11.107 & 0.038 & 1 & \\
\hline $02084+2038$ & CHE & 57 & 2016.913 & 93.4 & 0.6 & 5.290 & 0.023 & 2 & \\
\hline $02084+5514$ & STF & $217 \mathrm{BC}$ & 2016.037 & 142.2 & 0.2 & 32.089 & 0.109 & 1 & \\
\hline $02091+5104$ & STF & $213 \mathrm{AB}$ & 2016.880 & 326.0 & 0.2 & 1.896 & 0.006 & 1 & \\
\hline $02091+5515$ & STI & 1789 & 2016.037 & 318.8 & 0.2 & 7.199 & 0.024 & 1 & \\
\hline $02109+1341$ & $\mathrm{STF}$ & 224 & 2016.913 & 244.1 & 0.1 & 6.029 & 0.015 & 2 & \\
\hline $02109+3902$ & STF & 222 & 2016.883 & 36.5 & 0.2 & 16.627 & 0.057 & 1 & \\
\hline
\end{tabular}


Table 4-Continued

\begin{tabular}{|c|c|c|c|c|c|c|c|c|c|}
\hline $\begin{array}{l}\text { WDS Desig. } \\
\alpha, \delta(2000)\end{array}$ & $\begin{array}{l}\text { Dis } \\
\text { Desi }\end{array}$ & $\begin{array}{l}\text { coverer } \\
\text { gnation }\end{array}$ & JY & $\begin{array}{c}\theta \\
(0)\end{array}$ & $\begin{array}{l}\sigma \theta \\
(0)\end{array}$ & $\begin{array}{c}\rho \\
\left({ }^{\prime \prime}\right)\end{array}$ & $\begin{array}{l}\sigma \rho \\
\left({ }^{\prime \prime}\right)\end{array}$ & $\mathrm{n}$ & Note \\
\hline $02122+4440$ & HJ & $2117 \mathrm{AB}$ & 2016.880 & 35.0 & 0.2 & 8.669 & 0.029 & 1 & \\
\hline $02122+4440$ & HJ & $2117 \mathrm{AC}$ & 2016.880 & 194.0 & 0.2 & 13.372 & 0.045 & 1 & \\
\hline $02122+4440$ & HJ & $2117 \mathrm{AD}$ & 2016.880 & 286.0 & 0.2 & 23.192 & 0.079 & 1 & \\
\hline $02122+4440$ & HJ & $2117 \mathrm{BC}$ & 2016.880 & 202.1 & 0.2 & 21.698 & 0.074 & 1 & \\
\hline $02122+4440$ & HJ & $2117 \mathrm{BD}$ & 2016.880 & 268.6 & 0.2 & 27.266 & 0.093 & 1 & \\
\hline $02123+5554$ & STI & 1801 & 2016.880 & 46.6 & 0.2 & 12.516 & 0.043 & 1 & \\
\hline $02128-0224$ & STF & $231 \mathrm{AB}$ & 2016.010 & 235.0 & 0.2 & 16.855 & 0.057 & 1 & \\
\hline $02128+7941$ & $\mathrm{~S}$ & $405 \mathrm{AB}$ & 2016.853 & 277.8 & 0.2 & 55.647 & 0.189 & 1 & \\
\hline $02144+3810$ & ES & 230 & 2016.883 & 303.3 & 0.2 & 2.776 & 0.009 & 1 & \\
\hline $02150+5634$ & STI & 1817 & 2016.880 & 159.8 & 0.2 & 14.883 & 0.051 & 1 & \\
\hline $02154+1205$ & HJ & 22 & 2016.010 & 253.9 & 0.2 & 8.883 & 0.030 & 1 & \\
\hline $02172+3729$ & STF & $238 \mathrm{AC}$ & 2016.029 & 355.6 & 0.2 & 10.899 & 0.037 & 1 & \\
\hline $02172+5555$ & STF & 235 & 2016.880 & 48.6 & 0.2 & 1.897 & 0.006 & 1 & \\
\hline $02173+0457$ & BAL & 2599 & 2016.919 & 44.7 & 0.2 & 4.272 & 0.015 & 1 & \\
\hline $02176+5546$ & $\mathrm{BU}$ & 786 & 2016.880 & 349.6 & 0.2 & 5.218 & 0.018 & 1 & \\
\hline $02180+5616$ & $\mathrm{BRF}$ & $1 \mathrm{AB}$ & 2016.880 & 132.2 & 0.2 & 4.605 & 0.016 & 1 & \\
\hline $02180+5616$ & $\mathrm{PKO}$ & $25 \mathrm{AC}$ & 2016.880 & 25.6 & 0.2 & 19.133 & 0.065 & 1 & \\
\hline $02180+5616$ & $\mathrm{PKO}$ & $25 \mathrm{AD}$ & 2016.880 & 132.2 & 0.2 & 47.456 & 0.161 & 1 & \\
\hline $02180+5616$ & PKO & $25 \mathrm{BC}$ & 2016.880 & 13.4 & 0.2 & 20.906 & 0.071 & 1 & \\
\hline $02180+5616$ & $\mathrm{PKO}$ & $25 \mathrm{BD}$ & 2016.880 & 132.3 & 0.2 & 42.929 & 0.146 & 1 & \\
\hline $02180+5616$ & $\mathrm{PKO}$ & $25 \mathrm{CD}$ & 2016.880 & 151.1 & 0.2 & 56.211 & 0.191 & 1 & \\
\hline $02195+4300$ & $\mathrm{ROE}$ & 66 & 2016.883 & 188.6 & 0.2 & 6.085 & 0.021 & 1 & \\
\hline $02205+3415$ & $\mathrm{UC}$ & 720 & 2016.029 & 349.5 & 0.2 & 5.303 & 0.018 & 1 & \\
\hline $02213+3726$ & STF & 250 & 2016.883 & 136.7 & 0.2 & 3.149 & 0.011 & 1 & \\
\hline $02217+3923$ & STF & 251 & 2016.883 & 266.0 & 0.2 & 2.234 & 0.008 & 1 & \\
\hline $02217+7623$ & STF & 233 & 2016.853 & 273.3 & 0.2 & 1.951 & 0.007 & 1 & \\
\hline $02218+3414$ & $\mathrm{AG}$ & 37 & 2016.909 & 290.2 & 0.1 & 4.814 & 0.004 & 2 & \\
\hline $02220+5708$ & WFC & 8 & 2016.880 & 55.3 & 0.2 & 18.385 & 0.063 & 1 & \\
\hline $02229+3529$ & ALI & 32 & 2016.029 & 247.8 & 0.2 & 12.628 & 0.043 & 1 & \\
\hline $02234+4441$ & ES & 1306 & 2016.880 & 276.0 & 0.2 & 9.422 & 0.032 & 1 & \\
\hline $02235+2623$ & $\mathrm{KU}$ & 76 & 2016.894 & 349.9 & 0.0 & 32.021 & 0.015 & 2 & \\
\hline $02235+4047$ & GRV & 128 & 2016.883 & 206.8 & 0.2 & 36.282 & 0.123 & 1 & \\
\hline
\end{tabular}


Table 4-Continued

\begin{tabular}{|c|c|c|c|c|c|c|c|c|c|}
\hline \multirow{2}{*}{$\begin{array}{c}\text { WDS Desig. } \\
\alpha, \delta(2000) \\
02239+3330\end{array}$} & \multicolumn{2}{|c|}{$\begin{array}{c}\text { Discoverer } \\
\text { Designation }\end{array}$} & JY & \multirow{2}{*}{$\begin{array}{c}\theta \\
(0) \\
151.5\end{array}$} & \multirow{2}{*}{$\begin{array}{l}\sigma \theta \\
(\circ) \\
0.2\end{array}$} & \multirow{2}{*}{$\begin{array}{c}\rho \\
\left({ }^{\prime \prime}\right) \\
70.856\end{array}$} & \multirow{2}{*}{$\begin{array}{c}\begin{array}{c}\sigma \rho \\
\left({ }^{\prime \prime}\right.\end{array} \\
0.241\end{array}$} & \multirow{2}{*}{$\begin{array}{l}\mathrm{n} \\
\mathrm{C} \\
1\end{array}$} & \multirow{2}{*}{$\begin{array}{l}\text { Note } \\
\end{array}$} \\
\hline & STF & $258 \mathrm{AC}$ & 2016.883 & & & & & & \\
\hline $02239+3330$ & STF & $258 \mathrm{CD}$ & 2016.909 & 30.2 & 0.0 & 6.383 & 0.004 & 2 & \\
\hline $02242+5531$ & STI & 1865 & 2016.880 & 308.4 & 0.2 & 14.662 & 0.050 & 1 & \\
\hline $02244+1130$ & STF & 261 & 2016.933 & 253.3 & 0.1 & 2.872 & 0.003 & 2 & \\
\hline $02252+1217$ & BRT & 1170 & 2016.932 & 232.4 & 0.2 & 4.715 & 0.012 & 2 & \\
\hline $02252+3707$ & ES & 2551 & 2016.029 & 16.4 & 0.2 & 5.528 & 0.019 & 1 & \\
\hline $02261+4914$ & $\mathrm{HU}$ & 537 & 2016.880 & 21.8 & 0.2 & 2.131 & 0.007 & 1 & \\
\hline $02264-0540$ & HJ & 2138 & 2016.010 & 157.6 & 0.2 & 12.379 & 0.042 & 1 & \\
\hline $02279+3406$ & ES & 323 & 2016.029 & 190.7 & 0.2 & 6.987 & 0.024 & 1 & \\
\hline $02281+3251$ & ES & 2407 & 2016.909 & 282.7 & 0.0 & 6.713 & 0.010 & 2 & \\
\hline $02282+5423$ & HJ & $5535 \mathrm{CD}$ & 2016.818 & 145.1 & 0.2 & 19.654 & 0.067 & 1 & \\
\hline $02282+5423$ & STF & $267 \mathrm{AB}$ & 2016.818 & 28.6 & 0.2 & 94.003 & 0.320 & 1 & \\
\hline $02282+5423$ & STF & $267 \mathrm{BC}$ & 2016.818 & 25.5 & 0.2 & 69.448 & 0.236 & 1 & \\
\hline $02284+1722$ & A & 2330 & 2016.922 & 211.3 & 0.8 & 1.201 & 0.005 & 2 & \\
\hline $02288+4659$ & WFC & 10 & 2016.880 & 8.9 & 0.2 & 8.609 & 0.029 & 1 & \\
\hline $02292+5352$ & HJ & 2136 & 2016.818 & 36.0 & 0.2 & 4.711 & 0.016 & 1 & \\
\hline $02295+1537$ & HAM & 1 & 2016.029 & 194.8 & 0.2 & 4.431 & 0.015 & 1 & \\
\hline $02299+1540$ & SLE & 266 & 2016.029 & 93.3 & 0.2 & 18.521 & 0.063 & 1 & \\
\hline $02308+5533$ & STF & $270 \mathrm{AB}$ & 2016.880 & 305.6 & 0.2 & 21.267 & 0.072 & 1 & \\
\hline $02309+5311$ & HJ & $2139 \mathrm{AB}$ & 2016.818 & 297.4 & 0.2 & 3.935 & 0.013 & 1 & \\
\hline $02309+5311$ & HJ & $2139 \mathrm{AC}$ & 2016.818 & 8.2 & 0.2 & 18.076 & 0.061 & 1 & \\
\hline $02309+5311$ & HJ & $2139 \mathrm{AD}$ & 2016.818 & 203.4 & 0.2 & 59.464 & 0.202 & 1 & \\
\hline $02309+5311$ & HJ & $2139 \mathrm{BC}$ & 2016.818 & 20.6 & 0.2 & 17.090 & 0.058 & 1 & \\
\hline $02313+4703$ & $\mathrm{~A}$ & 968 & 2016.880 & 27.7 & 0.2 & 1.722 & 0.006 & 1 & \\
\hline $02316+1550$ & SLE & 267 & 2016.029 & 225.3 & 0.2 & 19.808 & 0.067 & 1 & \\
\hline $02319-0151$ & BAL & 14 & 2016.010 & 76.6 & 0.2 & 8.703 & 0.030 & 1 & \\
\hline $02320+1822$ & STF & 273 & 2016.913 & 359.5 & 0.1 & 7.185 & 0.009 & 2 & \\
\hline $02320+3452$ & PTT & 6 & 2016.029 & 234.3 & 0.2 & 5.801 & 0.020 & 1 & \\
\hline $02322+5415$ & HJ & $2142 \mathrm{AB}$ & 2016.818 & 312.0 & 0.2 & 8.161 & 0.028 & 1 & \\
\hline $02322+5415$ & HJ & $2142 \mathrm{AC}$ & 2016.818 & 350.6 & 0.2 & 8.639 & 0.029 & 1 & \\
\hline $02322+5415$ & HJ & $2142 \mathrm{BC}$ & 2016.818 & 56.5 & 0.2 & 5.582 & 0.019 & 1 & \\
\hline $02326+0935$ & HJ & 652 & 2016.893 & 315.8 & 0.2 & 4.193 & 0.010 & 2 & \\
\hline
\end{tabular}


Table 4-Continued

\begin{tabular}{|c|c|c|c|c|c|c|c|c|c|}
\hline $\begin{array}{l}\text { WDS Desig. } \\
\alpha, \delta(2000)\end{array}$ & $\begin{array}{l}\text { Dis } \\
\text { Desi }\end{array}$ & $\begin{array}{l}\text { coverer } \\
\text { gnation }\end{array}$ & JY & $\begin{array}{c}\theta \\
(0)\end{array}$ & $\begin{array}{l}\sigma \theta \\
(\circ)\end{array}$ & $\begin{array}{c}\rho \\
\left({ }^{\prime \prime}\right)\end{array}$ & $\begin{array}{l}\sigma \rho \\
\left({ }^{\prime \prime}\right)\end{array}$ & $\mathrm{n}$ & Note \\
\hline $02327+0620$ & STF & 276 & 2016.919 & 276.4 & 0.2 & 1.819 & 0.006 & 1 & \\
\hline $02330+4852$ & HJ & 2144 & 2016.880 & 259.9 & 0.2 & 26.955 & 0.092 & 1 & \\
\hline $02331+5828$ & STF & 272 & 2016.880 & 217.1 & 0.2 & 1.938 & 0.007 & 1 & \\
\hline $02332-0652$ & A & 449 & 2016.010 & 347.4 & 0.2 & 4.850 & 0.016 & 1 & \\
\hline $02338+4252$ & ES & 554 & 2016.883 & 0.2 & 0.2 & 6.148 & 0.021 & 1 & \\
\hline $02343+4017$ & $\mathrm{AG}$ & 42 & 2016.883 & 144.4 & 0.2 & 6.308 & 0.021 & 1 & \\
\hline $02351-1046$ & GAL & 323 & 2016.010 & 293.2 & 0.2 & 8.951 & 0.030 & 1 & \\
\hline $02357+4411$ & A & 1528 & 2016.850 & 194.2 & 0.2 & 1.536 & 0.005 & 1 & \\
\hline $02370+2439$ & STFA & $5 \mathrm{AB}$ & 2016.029 & 274.7 & 0.2 & 37.799 & 0.129 & 1 & \\
\hline $02374+5653$ & MLB & 1062 & 2016.875 & 176.8 & 0.2 & 8.624 & 0.029 & 1 & \\
\hline $02375+6258$ & MLB & 389 & 2016.051 & 124.0 & 0.2 & 4.326 & 0.015 & 1 & \\
\hline $02384+4213$ & ES & 555 & 2016.850 & 326.9 & 0.2 & 10.512 & 0.036 & 1 & \\
\hline $02385+0058$ & BAL & 959 & 2016.010 & 33.0 & 0.2 & 19.233 & 0.065 & 1 & \\
\hline $02388+1729$ & HJ & 2153 & 2016.850 & 353.5 & 0.0 & 19.825 & 0.012 & 3 & \\
\hline $02388+3325$ & STF & 285 & 2016.853 & 162.9 & 0.0 & 1.703 & 0.007 & 2 & \\
\hline $02389-0823$ & SKF & 768 & 2016.010 & 255.3 & 0.2 & 11.387 & 0.039 & 1 & \\
\hline $02389+1526$ & $\mathrm{AG}$ & 43 & 2016.870 & 62.7 & 0.2 & 2.971 & 0.005 & 3 & \\
\hline $02391+6006$ & STI & 376 & 2016.051 & 1.2 & 0.2 & 13.855 & 0.047 & 1 & \\
\hline $02397+0055$ & GRV & 138 & 2016.010 & 53.1 & 0.2 & 32.648 & 0.111 & 1 & \\
\hline $02402+0753$ & $\mathrm{AG}$ & 45 & 2016.960 & 341.2 & 0.2 & 4.657 & 0.018 & 2 & \\
\hline $02405+4535$ & ES & 1308 & 2016.850 & 276.5 & 0.2 & 6.951 & 0.024 & 1 & \\
\hline $02405+6129$ & STF & $283 \mathrm{AB}$ & 2016.875 & 210.1 & 0.2 & 1.802 & 0.006 & 1 & \\
\hline $02407+6117$ & STF & $284 \mathrm{AC}$ & 2016.875 & 9.9 & 0.2 & 53.088 & 0.180 & 1 & \\
\hline $02408+1500$ & $\mathrm{AG}$ & 47 & 2016.029 & 312.0 & 0.2 & 22.473 & 0.076 & 1 & \\
\hline $02412+4241$ & HJ & 2154 & 2016.850 & 140.8 & 0.2 & 9.972 & 0.034 & 1 & \\
\hline $02422+4242$ & STT & $44 \mathrm{AB}$ & 2016.850 & 55.4 & 0.2 & 1.369 & 0.005 & 1 & \\
\hline $02424+4246$ & HJ & $1126 \mathrm{AC}$ & 2016.850 & 98.3 & 0.2 & 56.815 & 0.193 & 1 & \\
\hline $02444+2856$ & MLB & 454 & 2016.853 & 233.8 & 0.2 & 5.294 & 0.018 & 1 & \\
\hline $02446+2928$ & STF & 300 & 2016.852 & 316.4 & 0.1 & 3.163 & 0.010 & 2 & \\
\hline $02447-0158$ & STF & 303 & 2016.010 & 181.1 & 0.2 & 5.694 & 0.019 & 1 & \\
\hline $02451+0026$ & RST & 4750 & 2004.959 & 158.3 & 0.2 & 3.245 & 0.011 & 1 & A \\
\hline $02455+0137$ & $\mathrm{UC}$ & 797 & 2016.010 & 199.4 & 0.2 & 4.353 & 0.015 & 1 & \\
\hline
\end{tabular}


Table 4-Continued

\begin{tabular}{|c|c|c|c|c|c|c|c|c|c|}
\hline $\begin{array}{l}\text { WDS Desig. } \\
\alpha, \delta(2000)\end{array}$ & & $\begin{array}{l}\text { iscoverer } \\
\text { signation }\end{array}$ & JY & $\begin{array}{c}\theta \\
(0)\end{array}$ & $\begin{array}{l}\sigma \theta \\
(0)\end{array}$ & $\begin{array}{c}\rho \\
\left({ }^{\prime \prime}\right)\end{array}$ & $\begin{array}{l}\sigma \rho \\
\left({ }^{\prime \prime}\right)\end{array}$ & $\mathrm{n}$ & Note \\
\hline $02469+1757$ & CHE & 68 & 2016.029 & 148.4 & 0.2 & 20.517 & 0.070 & 1 & \\
\hline $02470+4705$ & $\mathrm{AG}$ & $50 \mathrm{AB}$ & 2016.850 & 4.5 & 0.2 & 11.576 & 0.039 & 1 & \\
\hline $02470+4906$ & ES & 461 & 2016.850 & 342.0 & 0.2 & 7.751 & 0.026 & 1 & \\
\hline $02470+5007$ & $\mathrm{ARG}$ & 9 & 2016.850 & 152.4 & 0.2 & 2.567 & 0.009 & 1 & \\
\hline $02476+5357$ & STF & 301 & 2016.875 & 17.6 & 0.2 & 8.237 & 0.028 & 1 & \\
\hline $02477+1933$ & LDS & 881 & 2016.029 & 40.6 & 0.2 & 55.000 & 0.187 & 1 & \\
\hline $02477+6108$ & ES & 1876 & 2016.051 & 299.3 & 0.2 & 3.548 & 0.012 & 1 & \\
\hline $02480-0940$ & GAL & 325 & 2016.010 & 152.4 & 0.2 & 3.138 & 0.011 & 1 & \\
\hline $02495+1805$ & CHE & 71 & 2016.029 & 176.9 & 0.2 & 12.202 & 0.041 & 1 & \\
\hline $02497+1209$ & AG & $54 \mathrm{AB}$ & 2016.912 & 2.6 & 0.0 & 29.917 & 0.003 & 3 & \\
\hline $02499+0856$ & STF & 313 & 2016.963 & 194.6 & 0.1 & 5.708 & 0.009 & 2 & \\
\hline $02501+0138$ & BAL & 1257 & 2016.010 & 269.5 & 0.2 & 8.775 & 0.030 & 1 & \\
\hline $02501-0616$ & $\mathrm{~J}$ & $1453 \mathrm{AB}$ & 2016.010 & 200.4 & 0.2 & 9.237 & 0.031 & 1 & \\
\hline $02505+4118$ & ES & 1613 & 2016.850 & 19.4 & 0.2 & 6.874 & 0.023 & 1 & \\
\hline $02507+5700$ & $\mathrm{SCA}$ & 5 & 2016.037 & 37.6 & 0.2 & 16.025 & 0.054 & 1 & \\
\hline $02511+6025$ & STI & $398 \mathrm{EF}$ & 2016.037 & 91.9 & 0.2 & 8.722 & 0.030 & 1 & \\
\hline $02511+6025$ & STI & $398 \mathrm{FH}$ & 2016.037 & 106.6 & 0.2 & 14.548 & 0.049 & 1 & \\
\hline $02514+1736$ & $\mathrm{KU}$ & 79 & 2016.029 & 297.0 & 0.2 & 20.926 & 0.071 & 1 & \\
\hline $02516+6033$ & $\mathrm{BU}$ & $1374 \mathrm{AB}$ & 2016.875 & 196.3 & 0.2 & 21.072 & 0.072 & 1 & \\
\hline $02516+6033$ & $\mathrm{BU}$ & $1374 \mathrm{BH}$ & 2016.875 & 271.5 & 0.2 & 9.380 & 0.032 & 1 & \\
\hline $02518+5703$ & $\mathrm{SCA}$ & 6 & 2016.875 & 262.1 & 0.2 & 32.143 & 0.109 & 1 & \\
\hline $02523+0419$ & BAL & 2109 & 2016.971 & 234.1 & 0.2 & 6.513 & 0.022 & 1 & \\
\hline $02527+0628$ & STF & 323 & 2016.907 & 278.7 & 0.2 & 2.651 & 0.052 & 2 & \\
\hline $02529+1040$ & $\mathrm{AG}$ & 56 & 2016.888 & 290.4 & 0.0 & 6.827 & 0.005 & 3 & \\
\hline $02529+5300$ & STF & $314 \mathrm{AB}, \mathrm{C}$ & 2016.850 & 316.0 & 0.2 & 1.579 & 0.005 & 1 & \\
\hline $02533+5709$ & STI & 1948 & 2016.037 & 138.6 & 0.2 & 18.692 & 0.064 & 1 & \\
\hline $02538+3814$ & $\mathrm{~J}$ & 885 & 2016.853 & 273.9 & 0.2 & 4.249 & 0.014 & 1 & \\
\hline $02541+3603$ & STF & 322 & 2016.851 & 321.3 & 0.0 & 5.714 & 0.015 & 2 & \\
\hline $02542+6039$ & $\mathrm{ES}$ & $1815 \mathrm{AC}$ & 2016.875 & 53.2 & 0.2 & 16.029 & 0.054 & 1 & \\
\hline $02563+5852$ & STF & 321 & 2016.037 & 25.2 & 0.2 & 18.521 & 0.063 & 1 & \\
\hline $02563+7253$ & STF & $312 \mathrm{AC}$ & 2016.875 & 132.3 & 0.2 & 43.014 & 0.146 & 1 & \\
\hline $02570+1848$ & GRV & 153 & 2016.029 & 276.4 & 0.2 & 7.501 & 0.026 & 1 & \\
\hline
\end{tabular}


Table 4-Continued

\begin{tabular}{|c|c|c|c|c|c|c|c|c|c|}
\hline $\begin{array}{l}\text { WDS Desig. } \\
\alpha, \delta(2000)\end{array}$ & $\begin{array}{l}\text { Dis } \\
\text { Desi }\end{array}$ & $\begin{array}{l}\text { coverer } \\
\text { ignation }\end{array}$ & JY & $\begin{array}{c}\theta \\
(0)\end{array}$ & $\begin{array}{l}\sigma \theta \\
\text { (०) }\end{array}$ & $\begin{array}{c}\rho \\
\left({ }^{\prime \prime}\right)\end{array}$ & $\begin{array}{l}\sigma \rho \\
\left({ }^{\prime \prime}\right)\end{array}$ & $\mathrm{n}$ & Note \\
\hline $02574+6408$ & MLB & 189 & 2016.051 & 122.3 & 0.2 & 5.478 & 0.019 & 1 & \\
\hline $02579+0025$ & STF & 332 & 2016.010 & 53.5 & 0.2 & 12.032 & 0.041 & 1 & \\
\hline $02588+4115$ & BRT & 336 & 2016.850 & 253.9 & 0.2 & 5.427 & 0.018 & 1 & \\
\hline $02594+0639$ & STF & 334 & 2016.971 & 307.0 & 0.2 & 1.114 & 0.004 & 1 & \\
\hline $02594+6034$ & SS & $5 \mathrm{AH}$ & 2016.875 & 50.4 & 0.2 & 25.027 & 0.085 & 1 & \\
\hline $02594+6034$ & SS & $5 \mathrm{AK}$ & 2016.875 & 122.5 & 0.2 & 36.569 & 0.124 & 1 & \\
\hline $02595+4110$ & ES & 1510 & 2016.850 & 145.2 & 0.2 & 4.876 & 0.017 & 1 & \\
\hline $02596+0508$ & BAL & 2603 & 2016.971 & 214.1 & 0.2 & 6.000 & 0.020 & 1 & \\
\hline $02597+2013$ & $\mathrm{~J}$ & 234 & 2016.886 & 20.9 & 0.0 & 5.352 & 0.008 & 3 & \\
\hline $03003+1432$ & $\mathrm{AG}$ & 60 & 2016.908 & 160.0 & 0.0 & 6.434 & 0.004 & 3 & \\
\hline $03006+6548$ & MLR & 122 & 2016.875 & 331.4 & 0.2 & 6.235 & 0.021 & 1 & \\
\hline $03009+5221$ & STF & 331 & 2016.850 & 85.5 & 0.2 & 12.029 & 0.041 & 1 & \\
\hline $03013+6635$ & MLB & 392 & 2016.902 & 26.5 & 0.1 & 7.450 & 0.006 & 2 & \\
\hline $03018+5851$ & MLB & 53 & 2016.918 & 105.9 & 0.0 & 6.252 & 0.016 & 3 & \\
\hline $03038+7039$ & HJ & 2164 & 2016.920 & 320.5 & 0.1 & 5.536 & 0.011 & 2 & \\
\hline $03049+6439$ & MLB & 254 & 2016.051 & 193.8 & 0.2 & 6.559 & 0.022 & 1 & \\
\hline $03051+2755$ & STF & 342 & 2016.935 & 303.6 & 0.2 & 3.282 & 0.011 & 1 & \\
\hline $03053-0857$ & A & 456 & 2016.891 & 45.6 & 0.2 & 4.693 & 0.016 & 1 & \\
\hline $03068+7548$ & HJ & 2165 & 2016.906 & 212.4 & 0.1 & 39.025 & 0.042 & 3 & \\
\hline $03070+6744$ & HJ & 1131 & 2016.051 & 119.1 & 0.2 & 18.254 & 0.062 & 1 & \\
\hline $03081+2435$ & STF & 354 & 2016.935 & 52.1 & 0.2 & 35.583 & 0.121 & 1 & \\
\hline $03083-1236$ & STF & 357 & 2016.891 & 296.0 & 0.2 & 8.626 & 0.029 & 1 & \\
\hline $03083+7126$ & HJ & 2168 & 2016.051 & 297.9 & 0.2 & 18.499 & 0.063 & 1 & \\
\hline $03085-0335$ & FOX & $9023 \mathrm{CD}$ & 2016.891 & 155.7 & 0.2 & 17.423 & 0.059 & 1 & \\
\hline $03093+6849$ & WFC & 13 & 2016.968 & 288.3 & 0.1 & 7.971 & 0.025 & 2 & \\
\hline $03101+2537$ & BRT & 132 & 2004.058 & 200.5 & 0.2 & 4.772 & 0.016 & 1 & $\mathrm{~A}$ \\
\hline $03107+0010$ & BAL & 654 & 2016.029 & 225.1 & 0.2 & 5.918 & 0.020 & 1 & \\
\hline $03108+1508$ & GLP & 1 & 2016.935 & 89.4 & 0.2 & 4.530 & 0.015 & 1 & \\
\hline $03108+6347$ & STF & 349 & 2016.920 & 323.1 & 0.0 & 5.929 & 0.006 & 2 & \\
\hline $03112-0059$ & BAL & 282 & 2016.029 & 321.7 & 0.2 & 9.075 & 0.031 & 1 & \\
\hline $03115+1527$ & HEI & 28 & 2016.935 & 44.1 & 0.2 & 5.628 & 0.019 & 1 & \\
\hline $03146+6702$ & HJ & 1132 & 2016.956 & 24.8 & 0.0 & 7.243 & 0.004 & 2 & \\
\hline
\end{tabular}


Table 4-Continued

\begin{tabular}{|c|c|c|c|c|c|c|c|c|c|}
\hline $\begin{array}{l}\text { WDS Desig. } \\
\alpha, \delta(2000)\end{array}$ & $\begin{array}{l}\text { Disc } \\
\text { Desi }\end{array}$ & $\begin{array}{l}\text { coverer } \\
\text { ignation }\end{array}$ & JY & $\begin{array}{c}\theta \\
(0)\end{array}$ & $\begin{array}{l}\sigma \theta \\
(0)\end{array}$ & $\begin{array}{c}\rho \\
(")\end{array}$ & $\begin{array}{l}\sigma \rho \\
\left({ }^{\prime \prime}\right)\end{array}$ & $\mathrm{n}$ & Note \\
\hline $03148+6434$ & MLB & $333 \mathrm{AB}$ & 2016.051 & 309.4 & 0.2 & 5.030 & 0.017 & 1 & \\
\hline 03149-1400 & HJ & 3558 & 2016.891 & 139.6 & 0.2 & 15.620 & 0.053 & 1 & \\
\hline $03153+7340$ & VKI & 11 & 2016.051 & 21.8 & 0.2 & 7.023 & 0.024 & 1 & \\
\hline $03163+6002$ & STF & $362 \mathrm{AB}$ & 2016.916 & 143.1 & 0.1 & 7.131 & 0.009 & 3 & \\
\hline $03163+6002$ & STF & $362 \mathrm{AC}$ & 2016.924 & 46.4 & 0.0 & 26.639 & 0.054 & 2 & \\
\hline $03163+6002$ & STF & $362 \mathrm{AE}$ & 2016.884 & 243.2 & 0.1 & 35.136 & 0.009 & 2 & \\
\hline $03163+6002$ & STF & $362 \mathrm{BE}$ & 2016.884 & 254.8 & 0.2 & 37.026 & 0.046 & 2 & \\
\hline $03168+7830$ & STF & 345 & 2016.932 & 86.9 & 0.1 & 6.692 & 0.012 & 2 & \\
\hline $03183+1637$ & SLE & 39 & 2016.029 & 99.3 & 0.2 & 9.451 & 0.032 & 1 & \\
\hline $03183+5157$ & HJ & 2180 & 2016.037 & 296.0 & 0.2 & 34.935 & 0.119 & 1 & \\
\hline $03187+0404$ & BAL & 2114 & 2016.935 & 238.8 & 0.2 & 6.733 & 0.023 & 1 & \\
\hline $03190+5524$ & $\mathrm{SCA}$ & 14 & 2016.925 & 293.3 & 0.0 & 46.981 & 0.003 & 2 & \\
\hline $03192-0005$ & BAL & 657 & 2016.913 & 3.3 & 0.2 & 10.259 & 0.036 & 2 & \\
\hline $03195+5816$ & STI & 1974 & 2016.037 & 202.0 & 0.2 & 9.120 & 0.031 & 1 & \\
\hline $03203+1944$ & STF & 376 & 2016.935 & 252.0 & 0.2 & 7.152 & 0.024 & 1 & \\
\hline $03212+5910$ & STI & 1977 & 2016.936 & 70.6 & 0.1 & 5.637 & 0.006 & 3 & \\
\hline $03213+4743$ & ES & 464 & 2016.990 & 67.0 & 0.2 & 7.007 & 0.024 & 1 & \\
\hline $03221-1316$ & HJ & 3569 & 2016.891 & 212.4 & 0.2 & 19.468 & 0.066 & 1 & \\
\hline $03226+5559$ & HJ & 2185 & 2016.037 & 250.3 & 0.2 & 9.543 & 0.032 & 1 & \\
\hline $03240-1220$ & BRT & 2629 & 2004.959 & 18.9 & 0.2 & 7.047 & 0.024 & 1 & A \\
\hline $03242+1733$ & STF & 383 & 2016.935 & 120.7 & 0.2 & 5.569 & 0.019 & 1 & \\
\hline $03256+4701$ & $\mathrm{AG}$ & 306 & 2016.990 & 317.4 & 0.2 & 14.958 & 0.051 & 1 & \\
\hline $03280+2028$ & STF & $394 \mathrm{AB}$ & 2016.935 & 164.0 & 0.2 & 6.909 & 0.023 & 1 & \\
\hline $03280+5511$ & STF & 386 & 2016.894 & 59.8 & 0.1 & 2.700 & 0.001 & 2 & \\
\hline $03285+5954$ & STF & $384 \mathrm{AB}$ & 2016.910 & 273.5 & 0.2 & 1.960 & 0.007 & 3 & \\
\hline $03287+5026$ & STF & 388 & 2016.884 & 214.7 & 0.2 & 2.761 & 0.005 & 2 & \\
\hline 03289-1959 & I & $720 \mathrm{AB}$ & 2016.892 & 45.0 & 0.2 & 26.092 & 0.089 & 1 & \\
\hline $03299+1704$ & HJ & 3247 & 2016.029 & 201.2 & 0.2 & 6.901 & 0.023 & 1 & \\
\hline $03305+5451$ & STI & 1989 & 2016.037 & 179.9 & 0.2 & 9.835 & 0.033 & 1 & \\
\hline $03309+5558$ & $\mathrm{KR}$ & 20 & 2016.883 & 297.8 & 0.2 & 7.549 & 0.026 & 1 & \\
\hline $03313-0542$ & HLD & 65 & 2016.892 & 28.7 & 0.2 & 5.768 & 0.020 & 1 & \\
\hline $03314+5842$ & STI & 1991 & 2016.851 & 113.8 & 0.2 & 13.758 & 0.047 & 1 & \\
\hline
\end{tabular}


Table 4-Continued

\begin{tabular}{|c|c|c|c|c|c|c|c|c|c|}
\hline $\begin{array}{l}\text { WDS Desig. } \\
\alpha, \delta(2000)\end{array}$ & $\begin{array}{l}\text { Dis } \\
\text { Des }\end{array}$ & $\begin{array}{l}\text { scoverer } \\
\text { signation }\end{array}$ & JY & $\begin{array}{c}\theta \\
(0)\end{array}$ & $\begin{array}{l}\sigma \theta \\
(0)\end{array}$ & $\begin{array}{c}\rho \\
\left({ }^{\prime \prime}\right)\end{array}$ & $\begin{array}{l}\sigma \rho \\
\left({ }^{\prime \prime}\right)\end{array}$ & $\mathrm{n}$ & Note \\
\hline $03319+3536$ & ES & 2559 & 2004.053 & 291.2 & 0.2 & 7.576 & 0.026 & 1 & $\mathrm{~A}$ \\
\hline $03323+1613$ & $\mathrm{UC}$ & 50 & 2016.029 & 229.0 & 0.2 & 19.679 & 0.067 & 1 & \\
\hline $03335+5846$ & STF & $396 \mathrm{AB}$ & 2016.851 & 245.1 & 0.2 & 20.516 & 0.070 & 1 & \\
\hline $03340+6024$ & STF & 397 & 2016.883 & 42.8 & 0.2 & 5.196 & 0.018 & 1 & \\
\hline $03343+5817$ & STF & 398 & 2016.850 & 332.6 & 0.2 & 9.639 & 0.033 & 1 & \\
\hline $03343+6348$ & MLB & 190 & 2016.883 & 97.7 & 0.2 & 5.890 & 0.020 & 1 & \\
\hline $03351+6918$ & LDS & 1574 & 2016.051 & 205.1 & 0.2 & 26.717 & 0.091 & 1 & \\
\hline $03365-2038$ & CLL & 3 & 2016.892 & 206.4 & 0.2 & 42.680 & 0.145 & 1 & \\
\hline $03373+1803$ & HJ & 3249 & 2016.010 & 99.9 & 0.2 & 7.096 & 0.024 & 1 & \\
\hline $03380-0405$ & $\mathrm{UC}$ & 1034 & 2016.010 & 321.6 & 0.2 & 25.264 & 0.086 & 1 & \\
\hline $03383+0146$ & GRV & 182 & 2016.010 & 14.0 & 0.2 & 25.041 & 0.085 & 1 & \\
\hline $03400+6352$ & STTA & $36 \mathrm{~A}, \mathrm{BC}$ & 2016.883 & 71.7 & 0.2 & 45.852 & 0.156 & 1 & \\
\hline $03405+0508$ & $\mathrm{STF}$ & $430 \mathrm{AB}$ & 2016.010 & 56.1 & 0.2 & 26.207 & 0.089 & 1 & \\
\hline $03405+0508$ & STF & $430 \mathrm{AC}$ & 2016.010 & 299.0 & 0.2 & 34.272 & 0.117 & 1 & \\
\hline $03411+6039$ & ARG & 57 & 2016.883 & 317.1 & 0.2 & 6.035 & 0.021 & 1 & \\
\hline $03412+7319$ & HJ & 2193 & 2016.051 & 254.6 & 0.2 & 13.090 & 0.045 & 1 & \\
\hline $03414+7123$ & HJ & 1137 & 2016.051 & 37.7 & 0.2 & 12.865 & 0.044 & 1 & \\
\hline $03427+5958$ & WEB & $2 \mathrm{AD}$ & 2016.883 & 36.8 & 0.2 & 54.853 & 0.187 & 1 & \\
\hline $03427+6950$ & STF & $419 \mathrm{AB}$ & 2016.853 & 73.8 & 0.2 & 2.921 & 0.010 & 1 & \\
\hline $03428+0015$ & HJ & 2202 & 2016.010 & 78.3 & 0.2 & 33.721 & 0.115 & 1 & \\
\hline $03430+6407$ & STI & 476 & 2016.883 & 137.7 & 0.2 & 12.589 & 0.043 & 1 & \\
\hline 03459-2059 & ALG & 1 & 2016.892 & 293.8 & 0.2 & 5.761 & 0.020 & 1 & \\
\hline $03462+0022$ & BAL & 967 & 2016.010 & 219.8 & 0.2 & 16.682 & 0.057 & 1 & \\
\hline $03463-1235$ & GAL & 355 & 2016.892 & 347.1 & 0.2 & 32.181 & 0.109 & 1 & \\
\hline $03464-0810$ & HDS & 478 & 2016.892 & 348.1 & 0.2 & 7.480 & 0.025 & 1 & \\
\hline $03467-1231$ & GAL & 356 & 2016.892 & 76.9 & 0.2 & 26.307 & 0.089 & 1 & \\
\hline $03478+1504$ & GRV & 192 & 2016.010 & 27.6 & 0.2 & 40.492 & 0.138 & 1 & \\
\hline $03480+6840$ & KUI & $13 \mathrm{~A}, \mathrm{BC}$ & 2016.051 & 12.7 & 0.2 & 17.157 & 0.058 & 1 & \\
\hline $03493+0044$ & GRV & 193 & 2016.029 & 25.1 & 0.2 & 24.211 & 0.082 & 1 & \\
\hline $03493+5707$ & $\mathrm{~S}$ & 436 & 2016.850 & 76.8 & 0.2 & 58.238 & 0.198 & 1 & \\
\hline 03494-0115 & STE & $5 \mathrm{AC}$ & 2016.029 & 187.9 & 0.2 & 58.462 & 0.199 & 1 & \\
\hline $03507-1206$ & GAL & 358 & 2016.892 & 333.2 & 0.2 & 50.271 & 0.171 & 1 & \\
\hline
\end{tabular}


Table 4-Continued

\begin{tabular}{|c|c|c|c|c|c|c|c|c|c|}
\hline \multirow{2}{*}{$\begin{array}{l}\text { WDS Desig. } \\
\alpha, \delta(2000) \\
03507+6007\end{array}$} & \multicolumn{2}{|c|}{$\begin{array}{l}\text { Discoverer } \\
\text { Designation }\end{array}$} & JY & \multirow{2}{*}{$\begin{array}{c}\theta \\
(0) \\
259.2\end{array}$} & \multirow{2}{*}{$\begin{array}{l}\sigma \theta \\
(0) \\
0.2\end{array}$} & \multirow{2}{*}{$\begin{array}{c}\rho \\
\left({ }^{\prime \prime}\right) \\
3.111\end{array}$} & \multirow{2}{*}{$\begin{array}{l}\sigma \rho \\
\left({ }^{\prime \prime}\right) \\
0.011\end{array}$} & \multirow{2}{*}{$\mathrm{n}$} & \multirow{2}{*}{$\begin{array}{l}\text { Note } \\
\end{array}$} \\
\hline & STF & 445 & 2016.883 & & & & & & \\
\hline $03516+0020$ & STF & 463 & 2016.029 & 185.9 & 0.2 & 9.988 & 0.034 & 1 & \\
\hline $03520+3947$ & GRV & 197 & 2016.073 & 207.5 & 0.2 & 53.855 & 0.183 & 1 & \\
\hline $03520+5601$ & $\mathrm{KR}$ & 21 & 2016.883 & 279.2 & 0.2 & 4.184 & 0.014 & 1 & \\
\hline $03530+4112$ & $\mathrm{~A}$ & 1542 & 2016.073 & 289.3 & 0.2 & 4.928 & 0.017 & 1 & \\
\hline $03532-0200$ & STF & 466 & 2016.892 & 58.5 & 0.2 & 8.537 & 0.029 & 1 & \\
\hline $03535-1229$ & GAL & 359 & 2016.892 & 172.9 & 0.2 & 43.275 & 0.147 & 1 & \\
\hline $03541+7310$ & PRZ & 5 & 2016.051 & 238.4 & 0.2 & 30.207 & 0.103 & 1 & \\
\hline $03543-0257$ & STF & $470 \mathrm{AB}$ & 2016.892 & 348.8 & 0.0 & 6.905 & 0.056 & 2 & \\
\hline $03547+5200$ & TOR & 5 & 2016.037 & 313.0 & 0.2 & 7.439 & 0.025 & 1 & \\
\hline $03549+6345$ & STI & 479 & 2016.037 & 73.5 & 0.2 & 12.444 & 0.042 & 1 & \\
\hline $03558+5224$ & STF & 462 & 2016.037 & 319.9 & 0.2 & 8.077 & 0.027 & 1 & \\
\hline $03567+6344$ & MLB & 256 & 2016.037 & 274.1 & 0.2 & 4.328 & 0.015 & 1 & \\
\hline $03568+3828$ & STF & 467 & 2016.073 & 298.1 & 0.2 & 37.804 & 0.129 & 1 & \\
\hline $03568+5220$ & HJ & 5537 & 2016.037 & 76.3 & 0.2 & 18.967 & 0.064 & 1 & \\
\hline $03576+7748$ & HJ & 2203 & 2016.051 & 75.0 & 0.2 & 36.170 & 0.123 & 1 & \\
\hline $03597-1301$ & GAL & 361 & 2016.892 & 330.6 & 0.2 & 33.058 & 0.112 & 1 & \\
\hline $04005+7238$ & WFC & 19 & 2016.051 & 5.7 & 0.2 & 10.756 & 0.037 & 1 & \\
\hline $04066+4316$ & ES & 1520 & 2016.073 & 5.0 & 0.2 & 5.735 & 0.019 & 1 & \\
\hline $04066+7202$ & STF & $472 \mathrm{AB}$ & 2016.853 & 282.0 & 0.2 & 63.162 & 0.215 & 1 & \\
\hline $04066+7202$ & STF & $472 \mathrm{BC}$ & 2016.853 & 16.7 & 0.2 & 6.864 & 0.023 & 1 & \\
\hline $04069+4251$ & ES & 565 & 2016.073 & 26.6 & 0.2 & 6.363 & 0.022 & 1 & \\
\hline $04078+6220$ & HLM & $3 \mathrm{LM}$ & 2016.853 & 216.8 & 0.2 & 5.946 & 0.020 & 1 & \\
\hline $04078+6220$ & $\mathrm{HZG}$ & $2 \mathrm{LO}$ & 2016.853 & 93.3 & 0.2 & 42.356 & 0.144 & 1 & \\
\hline $04078+6220$ & $\mathrm{HZG}$ & $2 \mathrm{OP}$ & 2016.853 & 229.6 & 0.2 & 17.205 & 0.058 & 1 & \\
\hline $04078+6220$ & $\mathrm{STF}$ & $484 \mathrm{GH}$ & 2016.853 & 133.0 & 0.2 & 5.694 & 0.019 & 1 & \\
\hline $04078+6220$ & STF & 484 GI & 2016.853 & 336.4 & 0.2 & 22.750 & 0.077 & 1 & \\
\hline $04078+6220$ & STF & $484 \mathrm{HI}$ & 2016.853 & 331.7 & 0.2 & 27.992 & 0.095 & 1 & \\
\hline $04078+6220$ & STF & $485 \mathrm{AE}$ & 2016.853 & 305.9 & 0.2 & 17.978 & 0.061 & 1 & \\
\hline $04092+0126$ & $\mathrm{~J}$ & 236 & 2016.029 & 222.6 & 0.2 & 4.055 & 0.014 & 1 & \\
\hline $04100+6354$ & STI & 498 & 2016.051 & 85.0 & 0.2 & 7.253 & 0.025 & 1 & \\
\hline $04105+6009$ & STF & 490 & 2016.051 & 57.6 & 0.2 & 4.405 & 0.015 & 1 & \\
\hline
\end{tabular}


Table 4-Continued

\begin{tabular}{|c|c|c|c|c|c|c|c|c|c|}
\hline \multirow{2}{*}{$\begin{array}{c}\text { WDS Desig. } \\
\alpha, \delta(2000) \\
04106+2052\end{array}$} & \multicolumn{2}{|c|}{$\begin{array}{l}\text { Discoverer } \\
\text { Designation }\end{array}$} & \multirow{2}{*}{$\begin{array}{c}\text { JY } \\
2016.114\end{array}$} & \multirow{2}{*}{$\begin{array}{c}\theta \\
(0) \\
238.3\end{array}$} & \multirow{2}{*}{$\begin{array}{l}\sigma \theta \\
(0) \\
0.2\end{array}$} & \multirow{2}{*}{$\begin{array}{c}\rho \\
\left({ }^{\prime \prime}\right) \\
5.739\end{array}$} & \multirow{2}{*}{$\begin{array}{c}\begin{array}{c}\sigma \rho \\
\left({ }^{\prime \prime}\right)\end{array} \\
0.020\end{array}$} & \multirow{2}{*}{$\begin{array}{l}\mathrm{n} \\
\\
1\end{array}$} & \multirow{2}{*}{ Note } \\
\hline & RED & 29 & & & & & & & \\
\hline $04127-0650$ & STF & 514 & 2016.892 & 75.5 & 0.2 & 8.151 & 0.028 & 1 & \\
\hline $04140-1222$ & GAL & 364 & 2016.892 & 46.5 & 0.2 & 20.941 & 0.071 & 1 & \\
\hline $04144+6610$ & MLB & 396 & 2016.853 & 105.9 & 0.2 & 5.899 & 0.020 & 1 & \\
\hline $04159+3142$ & STTA & $43 \mathrm{AB}, \mathrm{C}$ & 2016.881 & 43.1 & 0.2 & 56.107 & 0.191 & 1 & \\
\hline $04180-0700$ & HJ & 23 & 2016.114 & 275.7 & 0.2 & 46.186 & 0.157 & 1 & \\
\hline $04183+6734$ & STF & 504 & 2016.881 & 266.6 & 0.2 & 6.800 & 0.023 & 1 & \\
\hline $04194+0623$ & HJ & $675 \mathrm{AB}$ & 2016.029 & 354.7 & 0.2 & 6.856 & 0.023 & 1 & \\
\hline $04194+0623$ & HJ & $675 \mathrm{AC}$ & 2016.029 & 104.1 & 0.2 & 11.245 & 0.038 & 1 & \\
\hline $04206+6146$ & STI & 509 & 2016.051 & 83.9 & 0.2 & 4.443 & 0.015 & 1 & \\
\hline $04209+7023$ & $\mathrm{STF}$ & 506 & 2016.853 & 295.9 & 0.2 & 9.785 & 0.033 & 1 & \\
\hline $04222+1844$ & SLE & 270 & 2016.029 & 98.7 & 0.2 & 10.957 & 0.037 & 1 & \\
\hline $04224+5149$ & $\mathrm{AG}$ & $310 \mathrm{AB}$ & 2016.990 & 25.5 & 0.2 & 21.381 & 0.073 & 1 & \\
\hline $04224+5149$ & SIN & $10 \mathrm{AE}$ & 2016.990 & 329.7 & 0.2 & 69.465 & 0.236 & 1 & \\
\hline $04225+5136$ & STF & 522 & 2016.990 & 212.1 & 0.2 & 1.507 & 0.005 & 1 & \\
\hline $04231+4934$ & STF & 524 & 2016.990 & 58.7 & 0.2 & 6.701 & 0.023 & 1 & \\
\hline $04234+1448$ & LDS & 5549 & 2016.029 & 64.1 & 0.2 & 5.109 & 0.017 & 1 & \\
\hline $04235+5358$ & $\mathrm{ES}$ & $956 \mathrm{AB}$ & 2016.990 & 292.3 & 0.2 & 2.159 & 0.007 & 1 & \\
\hline $04235+5358$ & ES & $956 \mathrm{AC}$ & 2016.990 & 181.4 & 0.2 & 27.784 & 0.094 & 1 & \\
\hline $04240+2418$ & STF & $534 \mathrm{AB}$ & 2016.114 & 290.6 & 0.2 & 29.195 & 0.099 & 1 & \\
\hline $04242+6048$ & STI & 511 & 2016.051 & 10.5 & 0.2 & 7.796 & 0.027 & 1 & \\
\hline $04260+4515$ & $\mathrm{ES}$ & $567 \mathrm{AB}$ & 2016.990 & 354.0 & 0.2 & 36.982 & 0.126 & 1 & \\
\hline $04260+4515$ & ES & $567 \mathrm{AC}$ & 2016.990 & 128.6 & 0.2 & 8.116 & 0.028 & 1 & \\
\hline $04271+1812$ & STF & $545 \mathrm{AB}$ & 2016.114 & 57.8 & 0.2 & 18.546 & 0.063 & 1 & \\
\hline $04279+2406$ & $\mathrm{POU}$ & 456 & 2016.114 & 92.3 & 0.2 & 8.450 & 0.029 & 1 & \\
\hline $04285+0505$ & HJ & 2233 & 2016.097 & 322.9 & 0.0 & 11.938 & 0.017 & 2 & \\
\hline $04292+0149$ & BAL & 1272 & 2016.029 & 346.9 & 0.2 & 11.478 & 0.039 & 1 & \\
\hline $04296+0350$ & BAL & 2121 & 2016.029 & 320.1 & 0.2 & 6.080 & 0.021 & 1 & \\
\hline $04304+6414$ & STF & $538 \mathrm{AB}$ & 2016.881 & 220.4 & 0.2 & 7.469 & 0.025 & 1 & \\
\hline $04304+6414$ & STF & $538 \mathrm{AC}$ & 2016.881 & 358.8 & 0.2 & 11.499 & 0.039 & 1 & \\
\hline $04304+6414$ & WAL & $31 \mathrm{DA}$ & 2016.881 & 125.1 & 0.2 & 88.451 & 0.301 & 1 & \\
\hline $04314+4001$ & STF & 552 & 2016.990 & 116.5 & 0.2 & 9.002 & 0.031 & 1 & \\
\hline
\end{tabular}


Table 4-Continued

\begin{tabular}{|c|c|c|c|c|c|c|c|c|c|}
\hline $\begin{array}{l}\text { WDS Desig. } \\
\alpha, \delta(2000)\end{array}$ & $\begin{array}{l}\text { Dis } \\
\text { Desi }\end{array}$ & $\begin{array}{l}\text { scoverer } \\
\text { ignation }\end{array}$ & JY & $\begin{array}{c}\theta \\
(\circ)\end{array}$ & $\begin{array}{l}\sigma \theta \\
(\circ)\end{array}$ & $\begin{array}{c}\rho \\
\left({ }^{\prime \prime}\right)\end{array}$ & $\begin{array}{l}\sigma \rho \\
\left({ }^{\prime \prime}\right)\end{array}$ & $\mathrm{n}$ & Note \\
\hline $04319+0749$ & BRT & 2596 & 2016.114 & 325.3 & 0.2 & 6.640 & 0.023 & 1 & \\
\hline $04320+5355$ & $\mathrm{STF}$ & $550 \mathrm{AB}$ & 2016.990 & 309.0 & 0.2 & 10.367 & 0.035 & 1 & \\
\hline $04329+0007$ & LDS & $121 \mathrm{AB}$ & 2016.029 & 323.0 & 0.2 & 16.872 & 0.057 & 1 & \\
\hline $04344+1630$ & $\mathrm{OL}$ & 107 & 2016.114 & 331.1 & 0.2 & 4.432 & 0.015 & 1 & \\
\hline $04344+6928$ & HJ & 1145 & 2016.051 & 131.9 & 0.2 & 4.461 & 0.015 & 1 & \\
\hline $04347+3824$ & $\mathrm{ES}$ & 2149 & 2016.990 & 220.3 & 0.2 & 5.895 & 0.020 & 1 & \\
\hline $04374+1852$ & LDS & 3600 & 2016.114 & 185.2 & 0.2 & 4.387 & 0.015 & 1 & \\
\hline $04378+0224$ & BAL & 1648 & 2016.029 & 338.4 & 0.2 & 15.509 & 0.053 & 1 & \\
\hline $04387-0024$ & $\mathrm{STF}$ & 575 & 2016.971 & 158.8 & 0.2 & 4.703 & 0.016 & 1 & \\
\hline $04393+2221$ & $\mathrm{KSA}$ & $25 \mathrm{AB}$ & 2016.114 & 184.4 & 0.2 & 28.057 & 0.095 & 1 & \\
\hline $04401+0319$ & $\mathrm{STF}$ & 578 & 2016.071 & 26.3 & 0.0 & 11.190 & 0.003 & 2 & \\
\hline $04408+2052$ & WSI & 43 & 2016.114 & 357.1 & 0.2 & 18.535 & 0.063 & 1 & \\
\hline $04426+5603$ & STI & 2065 & 2016.037 & 338.9 & 0.2 & 9.274 & 0.032 & 1 & \\
\hline $04429+5309$ & $\mathrm{STF}$ & 574 & 2016.990 & 315.5 & 0.2 & 4.555 & 0.015 & 1 & \\
\hline $04440+4225$ & $\mathrm{STF}$ & $581 \mathrm{CD}$ & 2016.990 & 342.8 & 0.2 & 8.070 & 0.027 & 1 & \\
\hline $04440+4225$ & $\mathrm{STF}$ & $582 \mathrm{AB}$ & 2016.990 & 23.5 & 0.2 & 5.674 & 0.019 & 1 & \\
\hline $04440+4225$ & TOB & $15 \mathrm{EF}$ & 2016.990 & 98.0 & 0.2 & 18.668 & 0.063 & 1 & \\
\hline $04441+5922$ & STI & 535 & 2016.037 & 231.7 & 0.2 & 11.705 & 0.040 & 1 & \\
\hline $04442+7249$ & $\mathrm{~A}$ & 838 & 2016.854 & 5.0 & 0.2 & 1.095 & 0.004 & 1 & \\
\hline $04450+4739$ & HJ & 2237 & 2016.037 & 125.2 & 0.2 & 16.809 & 0.057 & 1 & \\
\hline $04508+4608$ & SMA & 43 & 2016.037 & 83.9 & 0.2 & 13.133 & 0.045 & 1 & \\
\hline $04513+4901$ & $\mathrm{~J}$ & 650 & 2016.037 & 37.7 & 0.2 & 4.145 & 0.014 & 1 & \\
\hline $04527+5416$ & STI & 2074 & 2016.037 & 105.4 & 0.2 & 5.544 & 0.019 & 1 & \\
\hline $04542+6035$ & $\mathrm{STF}$ & 600 & 2016.854 & 55.0 & 0.2 & 29.760 & 0.101 & 1 & \\
\hline $04556+5539$ & STI & 2076 & 2016.037 & 224.2 & 0.2 & 15.571 & 0.053 & 1 & \\
\hline $04561+0411$ & BAL & 2621 & 2016.130 & 115.4 & 0.2 & 8.762 & 0.030 & 1 & \\
\hline $04570+0835$ & $\mathrm{AOT}$ & 18 & 2016.130 & 327.3 & 0.2 & 5.078 & 0.017 & 1 & \\
\hline $04576+1059$ & GRV & 222 & 2016.130 & 270.6 & 0.2 & 6.382 & 0.022 & 1 & \\
\hline $04578+4801$ & HJ & 2241 & 2016.037 & 84.1 & 0.2 & 11.919 & 0.041 & 1 & \\
\hline $04580+4902$ & ES & 1228 & 2016.037 & 304.9 & 0.2 & 6.045 & 0.021 & 1 & \\
\hline $04582+1652$ & GUI & 6 & 2016.130 & 253.4 & 0.2 & 5.546 & 0.019 & 1 & \\
\hline $04583+0424$ & BAL & 2623 & 2016.130 & 32.5 & 0.2 & 17.523 & 0.060 & 1 & \\
\hline
\end{tabular}


Table 4-Continued

\begin{tabular}{|c|c|c|c|c|c|c|c|c|c|}
\hline $\begin{array}{l}\text { WDS Desig. } \\
\alpha, \delta(2000)\end{array}$ & $\begin{array}{l}\text { Dis } \\
\text { Desi }\end{array}$ & $\begin{array}{l}\text { coverer } \\
\text { ignation }\end{array}$ & JY & $\begin{array}{c}\theta \\
(\circ)\end{array}$ & $\begin{array}{l}\sigma \theta \\
(\mathrm{o})\end{array}$ & $\begin{array}{c}\rho \\
\left({ }^{\prime \prime}\right)\end{array}$ & $\begin{array}{l}\sigma \rho \\
\left({ }^{\prime \prime}\right)\end{array}$ & $\mathrm{n}$ & Note \\
\hline $04586+6518$ & MLB & 397 & 2016.037 & 289.6 & 0.2 & 4.641 & 0.016 & 1 & \\
\hline $04590+1433$ & SHJ & $49 \mathrm{AB}$ & 2016.130 & 305.6 & 0.2 & 39.244 & 0.133 & 1 & \\
\hline $04590+1433$ & SHJ & $49 \mathrm{AC}$ & 2016.130 & 89.0 & 0.2 & 54.030 & 0.184 & 1 & \\
\hline $04590+6919$ & $\mathrm{STF}$ & 602 & 2016.037 & 136.8 & 0.2 & 29.009 & 0.099 & 1 & \\
\hline $04599+2720$ & $\mathrm{STF}$ & 623 & 2016.130 & 205.7 & 0.2 & 20.433 & 0.069 & 1 & \\
\hline $04599+5523$ & STI & 2080 & 2016.037 & 147.4 & 0.2 & 11.029 & 0.037 & 1 & \\
\hline $05000+7003$ & $\mathrm{STF}$ & 604 & 2016.854 & 39.6 & 0.2 & 2.072 & 0.007 & 1 & \\
\hline $05004+6925$ & $\mathrm{STF}$ & 606 & 2016.854 & 301.9 & 0.2 & 37.609 & 0.128 & 1 & \\
\hline $05036+6305$ & $\mathrm{STF}$ & $617 \mathrm{DE}$ & 2016.854 & 122.1 & 0.2 & 12.600 & 0.043 & 1 & \\
\hline $05036+6305$ & $\mathrm{STF}$ & $618 \mathrm{AB}$ & 2016.854 & 212.1 & 0.2 & 32.828 & 0.112 & 1 & \\
\hline $05046+2349$ & $\mathrm{POU}$ & 526 & 2016.130 & 187.3 & 0.2 & 6.141 & 0.021 & 1 & \\
\hline $05062+2131$ & $\mathrm{BRT}$ & 2321 & 2016.130 & 147.7 & 0.2 & 4.678 & 0.016 & 1 & \\
\hline $05075+6112$ & STI & 552 & 2016.854 & 42.9 & 0.2 & 11.826 & 0.040 & 1 & \\
\hline $05078+0904$ & SKF & 336 & 2016.130 & 198.9 & 0.2 & 17.772 & 0.060 & 1 & \\
\hline $05079+6849$ & HJ & 1152 & 2016.854 & 47.5 & 0.2 & 14.095 & 0.048 & 1 & \\
\hline $05089+6749$ & $\mathrm{HU}$ & 1096 & 2016.854 & 269.3 & 0.2 & 1.230 & 0.004 & 1 & \\
\hline $05094+2350$ & $\mathrm{POU}$ & 566 & 2016.130 & 291.4 & 0.2 & 10.769 & 0.037 & 1 & \\
\hline $05098+2411$ & $\mathrm{POU}$ & 569 & 2016.130 & 166.9 & 0.2 & 15.089 & 0.051 & 1 & \\
\hline $05123+0317$ & SKF & 339 & 2016.073 & 208.5 & 0.2 & 39.316 & 0.134 & 1 & \\
\hline $05123+6127$ & $\mathrm{HU}$ & 1098 & 2016.854 & 120.6 & 0.2 & 0.943 & 0.003 & 1 & \\
\hline $05127+6750$ & $\mathrm{STF}$ & 637 & 2016.854 & 36.8 & 0.2 & 20.683 & 0.070 & 1 & \\
\hline $05129-0347$ & $\mathrm{BRT}$ & 534 & 2016.127 & 172.6 & 0.2 & 4.097 & 0.014 & 1 & \\
\hline $05137-0137$ & BAL & 301 & 2016.127 & 134.9 & 0.2 & 19.964 & 0.068 & 1 & \\
\hline $05137-0856$ & MLL & 2 & 2016.127 & 260.4 & 0.2 & 5.117 & 0.017 & 1 & \\
\hline $05138+7528$ & $\mathrm{~A}$ & $842 \mathrm{AB}$ & 2016.854 & 282.7 & 0.2 & 45.898 & 0.156 & 1 & \\
\hline $05141-0317$ & $\mathrm{~J}$ & 324 & 2016.127 & 23.9 & 0.2 & 6.190 & 0.021 & 1 & \\
\hline $05142-0639$ & HDS & 693 & 2016.127 & 353.5 & 0.2 & 6.627 & 0.023 & 1 & \\
\hline $05143+2016$ & $\mathrm{HAU}$ & 12 & 2016.037 & 257.6 & 0.2 & 45.660 & 0.155 & 1 & \\
\hline $05143+6949$ & $\mathrm{STF}$ & 638 & 2016.854 & 222.4 & 0.2 & 5.202 & 0.018 & 1 & \\
\hline $05154-0322$ & BVD & 51 & 2016.127 & 317.4 & 0.2 & 44.481 & 0.151 & 1 & \\
\hline $05173+0557$ & $\mathrm{~J}$ & 241 & 2016.073 & 165.4 & 0.2 & 4.789 & 0.016 & 1 & \\
\hline $05175+0354$ & BAL & $2142 \mathrm{AB}$ & 2016.073 & 296.8 & 0.2 & 21.388 & 0.073 & 1 & \\
\hline
\end{tabular}


Table 4-Continued

\begin{tabular}{|c|c|c|c|c|c|c|c|c|c|}
\hline $\begin{array}{l}\text { WDS Desig. } \\
\alpha, \delta(2000)\end{array}$ & $\begin{array}{l}\text { Dis } \\
\text { Desi }\end{array}$ & $\begin{array}{l}\text { coverer } \\
\text { gnation }\end{array}$ & JY & $\begin{array}{c}\theta \\
(0)\end{array}$ & $\begin{array}{l}\sigma \theta \\
(0)\end{array}$ & $\begin{array}{c}\rho \\
\left({ }^{\prime \prime}\right)\end{array}$ & $\begin{array}{l}\sigma \rho \\
\left({ }^{\prime \prime}\right)\end{array}$ & $\mathrm{n}$ & Note \\
\hline $05175+0354$ & BAL & $2142 \mathrm{AC}$ & 2016.073 & 289.5 & 0.2 & 9.222 & 0.031 & 1 & B \\
\hline $05187-0115$ & BAL & 302 & 2016.127 & 348.4 & 0.2 & 14.132 & 0.048 & 1 & \\
\hline $05189+1757$ & $\mathrm{~J}$ & 2729 & 2016.073 & 358.8 & 0.2 & 6.538 & 0.022 & 1 & \\
\hline $05214-0653$ & MAD & $2 \mathrm{AB}$ & 2016.127 & 161.9 & 0.2 & 2.893 & 0.010 & 1 & \\
\hline $05214-0653$ & MAD & $2 \mathrm{AC}$ & 2016.127 & 136.9 & 0.2 & 35.635 & 0.121 & 1 & \\
\hline $05218+0418$ & BAL & 2632 & 2016.073 & 194.6 & 0.2 & 4.793 & 0.016 & 1 & \\
\hline $05233-0825$ & STF & 701 & 2016.127 & 138.5 & 0.2 & 6.287 & 0.021 & 1 & \\
\hline $05236+7038$ & HJ & 1155 & 2016.854 & 63.9 & 0.2 & 19.275 & 0.066 & 1 & \\
\hline 05240-0940 & GAL & 381 & 2016.127 & 296.9 & 0.2 & 29.549 & 0.100 & 1 & \\
\hline $05252-1119$ & STF & $710 \mathrm{AB}$ & 2016.127 & 195.9 & 0.2 & 10.709 & 0.036 & 1 & \\
\hline $05258+0207$ & BAL & 1668 & 2016.073 & 290.0 & 0.2 & 11.655 & 0.040 & 1 & \\
\hline $05267+0045$ & LEO & 57 & 2016.073 & 87.6 & 0.2 & 12.746 & 0.043 & 1 & \\
\hline $05268+0437$ & $\mathrm{BKO}$ & $15 \mathrm{AC}$ & 2016.073 & 177.9 & 0.2 & 12.864 & 0.044 & 1 & \\
\hline $05268+0437$ & BAL & $2634 \mathrm{AB}$ & 2016.073 & 203.1 & 0.2 & 17.004 & 0.058 & 1 & \\
\hline $05271+0044$ & DAM & $322 \mathrm{AE}$ & 2016.073 & 188.7 & 0.2 & 27.187 & 0.092 & 1 & \\
\hline $05284+8605$ & STF & $573 \mathrm{AB}$ & 2016.854 & 209.9 & 0.2 & 22.235 & 0.076 & 1 & \\
\hline $05293+2509$ & STF & $716 \mathrm{AB}$ & 2016.037 & 209.0 & 0.2 & 4.664 & 0.016 & 1 & \\
\hline $05303+1101$ & STF & 724 & 2016.990 & 253.4 & 0.2 & 6.819 & 0.023 & 1 & \\
\hline $05305+2223$ & $\mathrm{~J}$ & 590 & 2016.185 & 166.7 & 0.2 & 4.823 & 0.016 & 1 & \\
\hline $05309+1015$ & STF & 726 & 2016.990 & 263.8 & 0.2 & 1.150 & 0.004 & 1 & \\
\hline $05322+1703$ & STF & $730 \mathrm{AB}$ & 2016.990 & 141.5 & 0.2 & 9.681 & 0.033 & 1 & \\
\hline $05335+1601$ & STF & $733 \mathrm{AB}$ & 2016.990 & 41.1 & 0.2 & 12.416 & 0.042 & 1 & \\
\hline $05346+2137$ & GRV & 228 & 2016.990 & 352.5 & 0.2 & 11.999 & 0.041 & 1 & \\
\hline $05364+2111$ & STF & 740 & 2016.990 & 121.6 & 0.2 & 21.712 & 0.074 & 1 & \\
\hline $05365+2316$ & $\mathrm{POU}$ & 748 & 2016.185 & 46.4 & 0.2 & 10.359 & 0.035 & 1 & \\
\hline $05371+2655$ & BOW & $4 \mathrm{CD}$ & 2016.037 & 297.8 & 0.2 & 4.168 & 0.014 & 1 & \\
\hline $05386+2002$ & BRT & $2327 \mathrm{AB}$ & 2016.990 & 24.7 & 0.2 & 4.529 & 0.015 & 1 & \\
\hline $05387+1746$ & STF & $759 \mathrm{AB}$ & 2016.990 & 326.1 & 0.2 & 29.547 & 0.100 & 1 & \\
\hline $05387+1746$ & STF & $759 \mathrm{AC}$ & 2016.990 & 283.3 & 0.2 & 18.181 & 0.062 & 1 & \\
\hline $05388+1050$ & AHD & 24 & 2016.990 & 8.6 & 0.2 & 8.620 & 0.029 & 1 & \\
\hline $05391-0629$ & $\mathrm{UC}$ & 1339 & 2016.051 & 325.6 & 0.2 & 12.688 & 0.043 & 1 & \\
\hline $05393+1016$ & STF & $763 \mathrm{AB}$ & 2016.990 & 318.5 & 0.2 & 6.256 & 0.021 & 1 & \\
\hline
\end{tabular}


Table 4-Continued

\begin{tabular}{|c|c|c|c|c|c|c|c|c|c|}
\hline $\begin{array}{l}\text { WDS Desig. } \\
\alpha, \delta(2000)\end{array}$ & $\begin{array}{l}\text { Dis } \\
\text { Des }\end{array}$ & $\begin{array}{l}\text { coverer } \\
\text { ignation }\end{array}$ & JY & $\begin{array}{c}\theta \\
(0)\end{array}$ & $\begin{array}{l}\sigma \theta \\
(0)\end{array}$ & $\begin{array}{c}\rho \\
\left({ }^{\prime \prime}\right)\end{array}$ & $\begin{array}{l}\sigma \rho \\
\left({ }^{\prime \prime}\right)\end{array}$ & $\mathrm{n}$ & Note \\
\hline $05400+0315$ & BAL & 2155 & 2016.051 & 333.0 & 0.2 & 4.819 & 0.016 & 1 & \\
\hline $05400+2232$ & $\mathrm{AG}$ & 99 & 2016.990 & 144.0 & 0.2 & 7.609 & 0.026 & 1 & \\
\hline $05403+7401$ & STF & 714 & 2016.854 & 329.6 & 0.2 & 10.028 & 0.034 & 1 & \\
\hline $05413+2929$ & STF & 764 & 2016.037 & 14.2 & 0.2 & 25.956 & 0.088 & 1 & \\
\hline $05420+0951$ & GRV & 230 & 2016.051 & 340.7 & 0.2 & 15.294 & 0.052 & 1 & \\
\hline $05420+7139$ & STT & 109 & 2016.854 & 131.4 & 0.2 & 10.767 & 0.037 & 1 & \\
\hline $05424+7920$ & STF & $695 \mathrm{AB}$ & 2016.854 & 161.5 & 0.2 & 10.597 & 0.036 & 1 & \\
\hline $05440+2310$ & $\mathrm{POU}$ & 769 & 2016.051 & 88.9 & 0.2 & 14.719 & 0.050 & 1 & \\
\hline $05456-0128$ & BAL & 311 & 2016.051 & 28.6 & 0.2 & 12.985 & 0.044 & 1 & \\
\hline $05456-0644$ & $\mathrm{~J}$ & 332 & 2016.051 & 119.8 & 0.2 & 4.023 & 0.014 & 1 & \\
\hline $05462+2324$ & $\mathrm{POU}$ & 770 & 2016.051 & 74.4 & 0.2 & 13.851 & 0.047 & 1 & \\
\hline $05467+0005$ & $\mathrm{BU}$ & $559 \mathrm{AC}$ & 2016.114 & 202.4 & 0.2 & 50.444 & 0.172 & 1 & \\
\hline $05467+2350$ & $\mathrm{POU}$ & 774 & 2016.051 & 167.5 & 0.2 & 15.346 & 0.052 & 1 & \\
\hline $05479+1954$ & BRT & 2331 & 2016.114 & 204.7 & 0.2 & 4.918 & 0.017 & 1 & \\
\hline $05485+2342$ & HJ & 372 & 2016.051 & 204.6 & 0.2 & 15.978 & 0.054 & 1 & \\
\hline $05491+1758$ & $\mathrm{~J}$ & 1909 & 2016.051 & 29.4 & 0.2 & 5.297 & 0.018 & 1 & \\
\hline $05491+1904$ & BAR & $28 \mathrm{AB}$ & 2016.051 & 354.3 & 0.2 & 7.059 & 0.024 & 1 & \\
\hline $05503+0417$ & BAL & $2643 \mathrm{AB}$ & 2016.051 & 357.1 & 0.1 & 16.628 & 0.006 & 2 & \\
\hline $05508+1800$ & $\mathrm{~J}$ & 1910 & 2016.051 & 322.3 & 0.2 & 7.109 & 0.024 & 1 & \\
\hline $05509+1754$ & $\mathrm{~J}$ & 1911 & 2016.051 & 305.1 & 0.2 & 6.096 & 0.021 & 1 & \\
\hline $05511+1754$ & STF & $806 \mathrm{AC}$ & 2016.051 & 200.8 & 0.2 & 11.107 & 0.038 & 1 & \\
\hline $05512+0429$ & BAL & 2644 & 2016.051 & 356.0 & 0.2 & 18.145 & 0.062 & 1 & \\
\hline $05513+1917$ & $\mathrm{~J}$ & $1819 \mathrm{AB}$ & 2016.114 & 298.2 & 0.2 & 7.337 & 0.025 & 1 & \\
\hline $05518+1026$ & $\mathrm{AG}$ & 319 AB & 2016.051 & 354.5 & 0.2 & 36.076 & 0.123 & 1 & \\
\hline $05518+1026$ & $A G$ & $319 \mathrm{AC}$ & 2016.051 & 165.9 & 0.2 & 34.535 & 0.117 & 1 & \\
\hline $05541+0219$ & BAL & 1679 & 2016.073 & 229.8 & 0.2 & 5.153 & 0.018 & 1 & \\
\hline $05558+2302$ & $\mathrm{POU}$ & 818 & 2016.114 & 308.8 & 0.2 & 7.645 & 0.026 & 1 & \\
\hline $05563+1850$ & $\mathrm{~J}$ & $2394 \mathrm{AB}$ & 2016.106 & 166.2 & 0.0 & 30.937 & 0.034 & 2 & \\
\hline $05563+1850$ & $\mathrm{~J}$ & $2394 \mathrm{AC}$ & 2016.106 & 213.9 & 0.0 & 21.995 & 0.009 & 2 & \\
\hline $05573+0434$ & BAL & 2646 & 2016.073 & 117.6 & 0.2 & 11.236 & 0.038 & 1 & \\
\hline 05574-0201 & BAL & 48 & 2016.114 & 21.0 & 0.2 & 17.310 & 0.059 & 1 & \\
\hline $05588+2351$ & $\mathrm{~J}$ & 957 & 2016.073 & 185.0 & 0.2 & 4.136 & 0.014 & 1 & \\
\hline
\end{tabular}


Table 4-Continued

\begin{tabular}{|c|c|c|c|c|c|c|c|c|c|}
\hline $\begin{array}{l}\text { WDS Desig. } \\
\alpha, \delta(2000)\end{array}$ & $\begin{array}{l}\text { Dis } \\
\text { Desi }\end{array}$ & $\begin{array}{l}\text { coverer } \\
\text { gnation }\end{array}$ & JY & $\begin{array}{l}\theta \\
(0)\end{array}$ & $\begin{array}{l}\sigma \theta \\
(0)\end{array}$ & $\begin{array}{c}\rho \\
\left({ }^{\prime \prime}\right)\end{array}$ & $\begin{array}{l}\sigma \rho \\
\left({ }^{\prime \prime}\right)\end{array}$ & $\mathrm{n}$ & Note \\
\hline $05592+1306$ & BRT & 1187 & 2016.990 & 157.0 & 0.2 & 4.822 & 0.016 & 1 & \\
\hline $05596+1312$ & BRT & 1188 & 2016.990 & 355.4 & 0.2 & 5.405 & 0.018 & 1 & \\
\hline 06008-1209 & GWP & 728 & 2016.114 & 269.6 & 0.2 & 13.964 & 0.047 & 1 & \\
\hline $06015+2120$ & GRV & 706 & 2016.073 & 274.1 & 0.2 & 52.007 & 0.177 & 1 & \\
\hline $06022+0059$ & HJ & 2290 & 2016.073 & 118.2 & 0.2 & 14.502 & 0.049 & 1 & \\
\hline $06023+2240$ & BRT & 2339 & 2016.073 & 308.8 & 0.2 & 4.546 & 0.015 & 1 & \\
\hline $06026-0028$ & BAL & 678 & 2016.073 & 324.8 & 0.2 & 4.763 & 0.016 & 1 & \\
\hline $06043+2210$ & $\mathrm{COU}$ & $161 \mathrm{AC}$ & 2016.114 & 10.5 & 0.2 & 14.266 & 0.049 & 1 & \\
\hline 06049-0224 & BAL & 49 & 2016.114 & 93.1 & 0.2 & 16.143 & 0.055 & 1 & \\
\hline $06053+0527$ & $\mathrm{~J}$ & 2014 & 2016.073 & 306.0 & 0.2 & 9.954 & 0.034 & 1 & \\
\hline $06054-0238$ & BAL & 51 & 2016.114 & 255.2 & 0.2 & 6.837 & 0.023 & 1 & \\
\hline $06055-0233$ & XMI & 10 & 2016.114 & 122.7 & 0.2 & 19.657 & 0.067 & 1 & \\
\hline $06057+0053$ & BAL & 989 & 2016.073 & 174.2 & 0.2 & 4.479 & 0.015 & 1 & \\
\hline $06058-0724$ & HJ & 2293 & 2016.114 & 221.4 & 0.2 & 8.526 & 0.029 & 1 & \\
\hline $06058+1326$ & SLE & $833 \mathrm{AB}$ & 2016.990 & 344.5 & 0.2 & 38.677 & 0.132 & 1 & \\
\hline $06058+1326$ & SLE & $833 \mathrm{AC}$ & 2016.990 & 346.8 & 0.2 & 31.510 & 0.107 & 1 & \\
\hline $06061+0516$ & $\mathrm{~J}$ & 1368 & 2016.073 & 73.3 & 0.2 & 7.036 & 0.024 & 1 & \\
\hline $06063+1441$ & SLE & 834 & 2016.073 & 323.7 & 0.2 & 13.377 & 0.045 & 1 & \\
\hline $06067+0459$ & $\mathrm{HDO}$ & 81 & 2016.073 & 305.1 & 0.2 & 14.561 & 0.050 & 1 & \\
\hline $06071-0233$ & BAL & 52 & 2016.114 & 203.0 & 0.2 & 11.599 & 0.039 & 1 & \\
\hline $06077+1352$ & CLU & 1 & 2016.990 & 4.1 & 0.2 & 7.100 & 0.024 & 1 & \\
\hline 06078-0214 & BAL & 54 & 2016.114 & 18.8 & 0.2 & 7.659 & 0.026 & 1 & \\
\hline $06078+1436$ & SLE & 836 & 2016.073 & 192.0 & 0.2 & 8.370 & 0.028 & 1 & \\
\hline $06082+0927$ & $\mathrm{~J}$ & 2736 & 2016.037 & 206.0 & 0.2 & 5.906 & 0.020 & 1 & \\
\hline $06083+1400$ & ARN & $64 \mathrm{AC}$ & 2016.990 & 171.7 & 0.2 & 31.959 & 0.109 & 1 & \\
\hline $06083+1400$ & STF & $844 \mathrm{AB}$ & 2016.990 & 10.0 & 0.2 & 23.481 & 0.080 & 1 & \\
\hline $06085+1358$ & JRN & $23 \mathrm{EF}$ & 2016.163 & 171.3 & 0.2 & 21.411 & 0.073 & 1 & \\
\hline $06085+1358$ & JRN & $23 \mathrm{EH}$ & 2016.163 & 82.9 & 0.2 & 78.051 & 0.265 & 1 & \\
\hline $06085+1358$ & JRN & $23 \mathrm{JI}$ & 2016.163 & 90.1 & 0.2 & 43.472 & 0.148 & 1 & \\
\hline $06085+1358$ & JRN & $23 \mathrm{NI}$ & 2016.163 & 356.4 & 0.2 & 56.975 & 0.194 & 1 & \\
\hline $06085+1358$ & STF & $848 \mathrm{AB}$ & 2016.990 & 111.3 & 0.2 & 2.379 & 0.008 & 1 & \\
\hline $06085+1358$ & STF & $848 \mathrm{AD}$ & 2016.825 & 122.3 & 0.1 & 27.997 & 0.271 & 2 & \\
\hline
\end{tabular}


Table 4-Continued

\begin{tabular}{|c|c|c|c|c|c|c|c|c|c|}
\hline $\begin{array}{l}\text { WDS Desig. } \\
\alpha, \delta(2000)\end{array}$ & & $\begin{array}{l}\text { iscoverer } \\
\text { signation }\end{array}$ & JY & $\begin{array}{c}\theta \\
(\circ)\end{array}$ & $\begin{array}{l}\sigma \theta \\
(\circ)\end{array}$ & $\begin{array}{c}\rho \\
\left({ }^{\prime \prime}\right)\end{array}$ & $\begin{array}{l}\sigma \rho \\
\left({ }^{\prime \prime}\right)\end{array}$ & $\mathrm{n}$ & Note \\
\hline $06085+1358$ & $\mathrm{STF}$ & $848 \mathrm{AE}$ & 2016.577 & 184.7 & 0.2 & 43.108 & 0.116 & 2 & \\
\hline $06085+1358$ & $\mathrm{STF}$ & $848 \mathrm{BD}$ & 2016.990 & 123.4 & 0.2 & 25.942 & 0.088 & 1 & \\
\hline $06085+1358$ & $\mathrm{STF}$ & $848 \mathrm{DE}$ & 2016.163 & 223.3 & 0.2 & 38.852 & 0.132 & 1 & \\
\hline $06085+1358$ & $\mathrm{STF}$ & $848 \mathrm{DH}$ & 2016.163 & 110.3 & 0.2 & 54.007 & 0.184 & 1 & \\
\hline $06091+1350$ & SLE & 840 & 2016.163 & 199.7 & 0.2 & 26.817 & 0.091 & 1 & \\
\hline $06093+0258$ & BAL & 1683 & 2016.037 & 16.0 & 0.2 & 8.912 & 0.030 & 1 & \\
\hline $06095-0038$ & BAL & 680 & 2016.185 & 179.8 & 0.2 & 9.411 & 0.032 & 1 & \\
\hline $06095-0620$ & HJ & 2298 AB,C & 2016.185 & 92.6 & 0.2 & 44.327 & 0.151 & 1 & \\
\hline $06096+2153$ & BRT & 2345 & 2016.163 & 167.3 & 0.2 & 5.126 & 0.017 & 1 & \\
\hline $06098+0423$ & BAL & $2652 \mathrm{AB}$ & 2016.037 & 344.6 & 0.2 & 13.786 & 0.047 & 1 & \\
\hline $06103+1304$ & SLE & 843 & 2016.163 & 128.5 & 0.2 & 7.682 & 0.026 & 1 & \\
\hline $06106-0421$ & $\mathrm{~J}$ & 1923 & 2016.163 & 36.5 & 0.2 & 5.238 & 0.018 & 1 & \\
\hline $06109-0722$ & GCB & 15 & 2016.163 & 85.5 & 0.2 & 5.657 & 0.019 & 1 & \\
\hline 06110-0331 & HJ & 2299 AB & 2016.163 & 41.5 & 0.2 & 17.717 & 0.060 & 1 & \\
\hline $06110-0331$ & HJ & $2299 \mathrm{AC}$ & 2016.163 & 299.7 & 0.2 & 22.311 & 0.076 & 1 & \\
\hline $06114+0424$ & BAL & 2655 & 2016.037 & 229.5 & 0.2 & 16.397 & 0.056 & 1 & \\
\hline $06116+1350$ & SLE & 844 & 2016.163 & 66.9 & 0.2 & 7.238 & 0.025 & 1 & \\
\hline 06118-0033 & HJ & 722 & 2016.037 & 152.2 & 0.2 & 11.616 & 0.039 & 1 & \\
\hline $06118-0138$ & BAL & 314 & 2016.163 & 156.9 & 0.2 & 14.154 & 0.048 & 1 & \\
\hline $06120+1048$ & $\mathrm{BRT}$ & 1196 & 2016.163 & 79.6 & 0.2 & 4.156 & 0.014 & 1 & \\
\hline $06121+0527$ & HJ & 2301 & 2016.037 & 353.9 & 0.2 & 10.001 & 0.034 & 1 & \\
\hline $06122+0640$ & $\mathrm{~J}$ & 1925 & 2016.037 & 177.8 & 0.2 & 5.581 & 0.019 & 1 & \\
\hline $06126+1710$ & $\mathrm{~J}$ & 968 & 2016.163 & 173.9 & 0.2 & 4.597 & 0.016 & 1 & \\
\hline $06127+0925$ & SLE & 846 & 2016.037 & 15.7 & 0.2 & 22.162 & 0.075 & 1 & \\
\hline $06128+0906$ & SLE & 847 & 2016.037 & 6.4 & 0.2 & 12.658 & 0.043 & 1 & \\
\hline 06129-0609 & HJ & $36 \mathrm{AC}$ & 2016.185 & 210.6 & 0.2 & 22.747 & 0.077 & 1 & \\
\hline 06129-0609 & $\mathrm{J}$ & $717 \mathrm{AB}$ & 2016.185 & 286.0 & 0.2 & 2.607 & 0.009 & 1 & \\
\hline 06129-0609 & $\mathrm{J}$ & $717 \mathrm{BC}$ & 2016.185 & 203.7 & 0.2 & 22.184 & 0.075 & 1 & \\
\hline $06130+1330$ & $\mathrm{~J}$ & 1928 & 2016.185 & 108.1 & 0.2 & 4.764 & 0.016 & 1 & \\
\hline $06131+2132$ & $\mathrm{~J}$ & 3307 & 2016.163 & 322.4 & 0.2 & 5.639 & 0.019 & 1 & \\
\hline $06131+8537$ & $\mathrm{STF}$ & 703 & 2016.854 & 57.8 & 0.2 & 51.055 & 0.174 & 1 & \\
\hline $06132-0119$ & $\mathrm{~J}$ & 340 & 2016.037 & 342.4 & 0.2 & 4.485 & 0.015 & 1 & \\
\hline
\end{tabular}


Table 4-Continued

\begin{tabular}{|c|c|c|c|c|c|c|c|c|c|}
\hline $\begin{array}{l}\text { WDS Desig. } \\
\alpha, \delta(2000)\end{array}$ & & $\begin{array}{l}\text { coverer } \\
\text { ignation }\end{array}$ & JY & $\begin{array}{c}\theta \\
(0)\end{array}$ & $\begin{array}{l}\sigma \theta \\
(0)\end{array}$ & $\begin{array}{c}\rho \\
\left({ }^{\prime \prime}\right)\end{array}$ & $\begin{array}{l}\sigma \rho \\
\left({ }^{\prime \prime}\right)\end{array}$ & $\mathrm{n}$ & Note \\
\hline $06132+0456$ & BAL & 2658 & 2016.037 & 80.0 & 0.2 & 19.139 & 0.065 & 1 & \\
\hline $06134+0556$ & $\mathrm{~J}$ & 969 & 2016.163 & 301.0 & 0.2 & 4.458 & 0.015 & 1 & \\
\hline $06137+0257$ & BAL & 1685 & 2016.163 & 35.5 & 0.2 & 5.016 & 0.017 & 1 & \\
\hline $06138+0846$ & XMI & 12 & 2016.037 & 204.7 & 0.2 & 14.996 & 0.051 & 1 & \\
\hline $06141+1058$ & $\mathrm{~J}$ & 1930 & 2016.037 & 277.2 & 0.2 & 7.282 & 0.025 & 1 & \\
\hline $06141+2129$ & BRT & 2347 & 2016.163 & 319.6 & 0.2 & 4.082 & 0.014 & 1 & \\
\hline $06142+1124$ & BPM & 257 & 2016.037 & 230.1 & 0.2 & 22.994 & 0.078 & 1 & \\
\hline $06146-0248$ & $\mathrm{BU}$ & 1407 & 2016.037 & 31.8 & 0.2 & 8.459 & 0.029 & 1 & \\
\hline $06150+0248$ & BAL & 1686 & 2016.037 & 209.7 & 0.2 & 16.495 & 0.056 & 1 & \\
\hline 06154-0152 & BAL & 317 & 2016.163 & 287.8 & 0.2 & 14.845 & 0.050 & 1 & \\
\hline $06155+1157$ & $\mathrm{~J}$ & 1363 & 2016.163 & 193.6 & 0.2 & 5.381 & 0.018 & 1 & \\
\hline 06156-0011 & BAL & 689 & 2016.037 & 311.8 & 0.2 & 13.381 & 0.045 & 1 & \\
\hline $06159+0000$ & BAL & $996 \mathrm{AC}$ & 2016.168 & 244.7 & 0.2 & 14.640 & 0.050 & 1 & \\
\hline $06165+0042$ & HJ & 724 & 2016.168 & 346.6 & 0.2 & 13.155 & 0.045 & 1 & \\
\hline $06166+1013$ & SLE & $857 \mathrm{AB}$ & 2016.168 & 314.3 & 0.2 & 14.001 & 0.048 & 1 & \\
\hline $06166+1013$ & SLE & $857 \mathrm{AC}$ & 2016.168 & 246.3 & 0.2 & 10.712 & 0.036 & 1 & \\
\hline $06166+1548$ & XMI & 13 & 2016.168 & 204.3 & 0.2 & 18.712 & 0.064 & 1 & \\
\hline $06168+0017$ & BAL & 999 & 2016.168 & 96.5 & 0.2 & 6.094 & 0.021 & 1 & \\
\hline $06169+1005$ & SLE & 858 & 2016.168 & 309.3 & 0.2 & 14.746 & 0.050 & 1 & \\
\hline $06176-0620$ & HJ & 37 & 2016.051 & 280.1 & 0.2 & 20.386 & 0.069 & 1 & \\
\hline $06176+2016$ & HJ & 2306 & 2016.169 & 34.0 & 0.2 & 4.089 & 0.014 & 1 & \\
\hline $06178-0414$ & HJ & 2310 & 2016.185 & 248.3 & 0.2 & 24.036 & 0.082 & 1 & \\
\hline $06178+1006$ & SLE & 864 & 2016.130 & 205.9 & 0.2 & 19.438 & 0.066 & 1 & \\
\hline $06181+0037$ & BAL & 1000 & 2016.168 & 268.0 & 0.2 & 11.003 & 0.037 & 1 & \\
\hline $06185-0042$ & BAL & 692 & 2016.168 & 298.7 & 0.2 & 13.189 & 0.045 & 1 & \\
\hline $06193-0121$ & BAL & 318 & 2016.051 & 357.7 & 0.2 & 13.151 & 0.045 & 1 & \\
\hline 06193-1232 & $\mathrm{HO}$ & 231 & 2016.185 & 48.0 & 0.2 & 6.800 & 0.023 & 1 & \\
\hline 06194-0820 & BRT & 377 & 2016.051 & 86.3 & 0.2 & 3.474 & 0.012 & 1 & \\
\hline $06195+1952$ & SCA & 47 & 2016.168 & 193.7 & 0.2 & 18.406 & 0.063 & 1 & \\
\hline $06198-0431$ & FAL & 15 & 2016.185 & 98.5 & 0.2 & 15.428 & 0.052 & 1 & \\
\hline $06198+2021$ & SCA & 48 & 2016.169 & 134.6 & 0.2 & 26.764 & 0.091 & 1 & \\
\hline $06199+1026$ & SLE & 871 & 2016.130 & 233.3 & 0.2 & 15.885 & 0.054 & 1 & \\
\hline
\end{tabular}


Table 4-Continued

\begin{tabular}{|c|c|c|c|c|c|c|c|c|c|}
\hline $\begin{array}{l}\text { WDS Desig. } \\
\alpha, \delta(2000)\end{array}$ & $\begin{array}{l}\text { Dis } \\
\text { Desi }\end{array}$ & $\begin{array}{l}\text { coverer } \\
\text { ignation }\end{array}$ & JY & $\begin{array}{c}\theta \\
(0)\end{array}$ & $\begin{array}{l}\sigma \theta \\
(0)\end{array}$ & $\begin{array}{c}\rho \\
\left({ }^{\prime \prime}\right)\end{array}$ & $\begin{array}{l}\sigma \rho \\
\left({ }^{\prime \prime}\right)\end{array}$ & $\mathrm{n}$ & Note \\
\hline $06200+0958$ & SLE & 872 & 2016.130 & 106.9 & 0.2 & 12.426 & 0.042 & 1 & \\
\hline $06201+1037$ & $\mathrm{~J}$ & 344 & 2016.168 & 95.0 & 0.2 & 3.729 & 0.013 & 1 & \\
\hline $06201+1143$ & $\mathrm{AG}$ & 324 & 2016.168 & 268.5 & 0.2 & 9.919 & 0.034 & 1 & \\
\hline $06202-0026$ & BAL & 694 & 2016.168 & 77.0 & 0.2 & 15.774 & 0.054 & 1 & \\
\hline $06205+1037$ & SLE & 873 & 2016.130 & 170.0 & 0.2 & 17.846 & 0.061 & 1 & \\
\hline $06208+0223$ & BAL & 1689 & 2016.130 & 46.5 & 0.2 & 9.951 & 0.034 & 1 & \\
\hline $06208+0849$ & $\mathrm{~J}$ & $409 \mathrm{AB}$ & 2016.130 & 134.9 & 0.2 & 4.990 & 0.017 & 1 & \\
\hline $06208+8411$ & STF & 784 & 2016.854 & 209.3 & 0.2 & 1.197 & 0.004 & 1 & \\
\hline 06209-0718 & HJ & 2315 & 2016.051 & 1.2 & 0.2 & 6.067 & 0.021 & 1 & \\
\hline $06210+0944$ & HJ & $725 \mathrm{AB}$ & 2016.130 & 70.4 & 0.2 & 23.990 & 0.082 & 1 & \\
\hline $06212+0259$ & BAL & 2163 & 2016.130 & 247.8 & 0.2 & 5.901 & 0.020 & 1 & \\
\hline $06212+0652$ & $\mathrm{~J}$ & 2003 & 2016.130 & 209.6 & 0.2 & 7.645 & 0.026 & 1 & \\
\hline $06214+0945$ & XMI & 14 & 2016.130 & 244.9 & 0.2 & 20.122 & 0.068 & 1 & \\
\hline $06214+1005$ & SLE & 876 & 2016.130 & 75.2 & 0.2 & 11.195 & 0.038 & 1 & \\
\hline $06220+0106$ & BAL & 1311 & 2016.168 & 6.1 & 0.2 & 17.509 & 0.060 & 1 & \\
\hline $06220+1058$ & STF & $898 \mathrm{AB}$ & 2016.130 & 122.2 & 0.1 & 6.027 & 0.037 & 2 & \\
\hline $06220+1058$ & WAL & $44 \mathrm{AC}$ & 2016.130 & 325.7 & 0.2 & 26.481 & 0.090 & 1 & \\
\hline $06221+0341$ & BAL & 2164 & 2016.168 & 204.2 & 0.2 & 4.599 & 0.016 & 1 & \\
\hline $06221+2203$ & $\mathrm{~L}$ & 59 & 2016.169 & 132.6 & 0.2 & 5.689 & 0.019 & 1 & \\
\hline $06226+0751$ & $\mathrm{~J}$ & 2020 & 2016.168 & 266.3 & 0.2 & 3.784 & 0.013 & 1 & \\
\hline $06232+0854$ & $\mathrm{ABH}$ & $44 \mathrm{AD}$ & 2016.130 & 158.1 & 0.2 & 14.133 & 0.048 & 1 & \\
\hline $06232+0854$ & HJ & $726 \mathrm{AB}$ & 2016.130 & 154.5 & 0.2 & 22.274 & 0.076 & 1 & \\
\hline $06232+0854$ & HJ & $726 \mathrm{AC}$ & 2016.130 & 87.2 & 0.2 & 23.092 & 0.079 & 1 & \\
\hline $06233+0353$ & BAL & 2165 & 2016.130 & 60.7 & 0.2 & 14.375 & 0.049 & 1 & \\
\hline $06235+0045$ & BAL & 1001 & 2016.051 & 138.2 & 0.2 & 15.519 & 0.053 & 1 & \\
\hline $06238+0436$ & $\mathrm{STF}$ & $900 \mathrm{AB}$ & 2016.130 & 27.9 & 0.2 & 11.952 & 0.041 & 1 & \\
\hline $06244+0519$ & HDS & 874 & 2016.158 & 192.1 & 0.2 & 9.365 & 0.032 & 1 & \\
\hline $06258+0950$ & $\mathrm{~J}$ & 1364 & 2016.158 & 236.4 & 0.2 & 5.807 & 0.020 & 1 & \\
\hline $06259+0655$ & GRV & 716 & 2016.158 & 176.4 & 0.2 & 32.831 & 0.112 & 1 & \\
\hline $06264+1128$ & $\mathrm{~J}$ & $595 \mathrm{AB}$ & 2016.157 & 41.9 & 0.2 & 4.754 & 0.016 & 1 & \\
\hline $06264+1128$ & $\mathrm{~J}$ & $595 \mathrm{AC}$ & 2016.157 & 244.7 & 0.2 & 43.736 & 0.149 & 1 & \\
\hline $06272+1118$ & $\mathrm{~J}$ & 1945 & 2016.157 & 93.3 & 0.2 & 7.591 & 0.026 & 1 & \\
\hline
\end{tabular}


Table 4-Continued

\begin{tabular}{|c|c|c|c|c|c|c|c|c|c|}
\hline $\begin{array}{l}\text { WDS Desig. } \\
\alpha, \delta(2000)\end{array}$ & $\begin{array}{l}\text { Dis } \\
\text { Desi }\end{array}$ & $\begin{array}{l}\text { coverer } \\
\text { ignation }\end{array}$ & JY & $\begin{array}{c}\theta \\
(\circ)\end{array}$ & $\begin{array}{l}\sigma \theta \\
\text { (०) }\end{array}$ & $\begin{array}{c}\rho \\
\left({ }^{\prime \prime}\right)\end{array}$ & $\begin{array}{l}\sigma \rho \\
(")\end{array}$ & $\mathrm{n}$ & Note \\
\hline $06273+0916$ & $\mathrm{~J}$ & 2744 & 2016.157 & 197.3 & 0.2 & 6.278 & 0.021 & 1 & \\
\hline $06296+0529$ & SLE & 286 & 2016.158 & 54.2 & 0.2 & 17.285 & 0.059 & 1 & \\
\hline $06296+0542$ & SLE & 287 & 2016.158 & 209.4 & 0.2 & 8.212 & 0.028 & 1 & \\
\hline $06296+7258$ & HJ & $2309 \mathrm{AC}$ & 2016.854 & 233.2 & 0.2 & 54.388 & 0.185 & 1 & \\
\hline $06299+1242$ & XMI & 16 & 2016.157 & 301.7 & 0.2 & 12.588 & 0.043 & 1 & \\
\hline $06302+1236$ & HJ & 3283 & 2016.157 & 355.2 & 0.2 & 11.739 & 0.040 & 1 & \\
\hline $06305+0420$ & STF & $920 \mathrm{AB}$ & 2016.158 & 211.0 & 0.2 & 9.465 & 0.032 & 1 & \\
\hline $06308+0518$ & SLE & 289 & 2016.158 & 286.7 & 0.2 & 9.931 & 0.034 & 1 & \\
\hline $06308+1329$ & $\mathrm{~J}$ & 1950 & 2016.157 & 168.1 & 0.2 & 7.050 & 0.024 & 1 & \\
\hline $06315+1535$ & BRT & 1204 & 2016.157 & 77.9 & 0.2 & 5.247 & 0.018 & 1 & \\
\hline $06319+0457$ & SLE & $293 \mathrm{GH}$ & 2016.158 & 274.8 & 0.2 & 18.318 & 0.062 & 1 & B \\
\hline $06319+0457$ & SLE & 293 KJ & 2016.158 & 359.7 & 0.2 & 36.332 & 0.124 & 1 & \\
\hline $06322+0600$ & SLE & 294 & 2016.158 & 250.4 & 0.2 & 16.324 & 0.056 & 1 & \\
\hline $06439+2512$ & BRT & 3277 & 2004.154 & 310.6 & 0.2 & 3.603 & 0.012 & 1 & $\mathrm{~A}$ \\
\hline $06539+0015$ & BAL & $1054 \mathrm{AB}$ & 2016.204 & 17.0 & 0.2 & 19.857 & 0.068 & 1 & \\
\hline $06539+0015$ & BAL & $1054 \mathrm{AC}$ & 2016.204 & 35.0 & 0.2 & 27.260 & 0.093 & 1 & \\
\hline $06539+0015$ & BAL & $1054 \mathrm{BC}$ & 2016.204 & 71.0 & 0.2 & 10.244 & 0.035 & 1 & \\
\hline $06541+0041$ & BAL & 1056 & 2016.204 & 105.1 & 0.2 & 16.531 & 0.056 & 1 & \\
\hline $06542+0128$ & BAL & 1357 & 2016.204 & 189.5 & 0.2 & 11.349 & 0.039 & 1 & \\
\hline $06543+1238$ & $\mathrm{BPM}$ & 336 & 2016.212 & 180.6 & 0.2 & 54.640 & 0.186 & 1 & \\
\hline 06544-0008 & $\mathrm{J}$ & $2616 \mathrm{AB}$ & 2016.204 & 3.3 & 0.2 & 5.089 & 0.017 & 1 & \\
\hline $06544+0051$ & BAL & 1058 & 2016.204 & 159.1 & 0.2 & 18.666 & 0.063 & 1 & \\
\hline 06544-0139 & FIL & 16 & 2016.204 & 167.1 & 0.2 & 10.861 & 0.037 & 1 & \\
\hline $06544+0146$ & BAL & 1358 & 2016.204 & 6.5 & 0.2 & 15.549 & 0.053 & 1 & \\
\hline $06544+1817$ & DOO & 38 & 2016.212 & 211.9 & 0.2 & 19.617 & 0.067 & 1 & \\
\hline $06546+0237$ & $\mathrm{AG}$ & 124 & 2016.204 & 204.5 & 0.2 & 6.448 & 0.022 & 1 & \\
\hline $06548+0334$ & STF & $989 \mathrm{AB}$ & 2016.204 & 215.8 & 0.2 & 8.566 & 0.029 & 1 & \\
\hline $06548+0334$ & STF & $989 \mathrm{AC}$ & 2016.204 & 85.0 & 0.2 & 14.880 & 0.051 & 1 & \\
\hline $06548+0334$ & STF & $989 \mathrm{BC}$ & 2016.204 & 67.5 & 0.2 & 21.415 & 0.073 & 1 & \\
\hline $06550-0142$ & BAL & 360 & 2016.204 & 39.1 & 0.2 & 7.433 & 0.025 & 1 & \\
\hline $06550+0246$ & BAL & 1741 & 2016.204 & 91.7 & 0.2 & 9.472 & 0.032 & 1 & \\
\hline $06552-0030$ & BAL & 737 & 2016.204 & 312.1 & 0.2 & 7.425 & 0.025 & 1 & \\
\hline
\end{tabular}


Table 4-Continued

\begin{tabular}{|c|c|c|c|c|c|c|c|c|c|}
\hline $\begin{array}{l}\text { WDS Desig. } \\
\alpha, \delta(2000)\end{array}$ & $\begin{array}{l}\text { Di } \\
\text { Des }\end{array}$ & $\begin{array}{l}\text { coverer } \\
\text { ignation }\end{array}$ & JY & $\begin{array}{c}\theta \\
(0)\end{array}$ & $\begin{array}{l}\sigma \theta \\
(0)\end{array}$ & $\begin{array}{c}\rho \\
\left({ }^{\prime \prime}\right)\end{array}$ & $\begin{array}{l}\sigma \rho \\
\left({ }^{\prime \prime}\right)\end{array}$ & $\mathrm{n}$ & Note \\
\hline $06552+0213$ & BAL & 1742 & 2016.204 & 245.0 & 0.2 & 4.455 & 0.015 & 1 & \\
\hline $06554-0149$ & BAL & 361 & 2016.204 & 291.0 & 0.2 & 12.652 & 0.043 & 1 & \\
\hline $06555-0150$ & BAL & 362 & 2016.204 & 340.8 & 0.2 & 17.774 & 0.060 & 1 & \\
\hline $06555+0234$ & $\mathrm{AG}$ & 125 & 2016.204 & 251.9 & 0.2 & 15.005 & 0.051 & 1 & \\
\hline $06556-0058$ & BAL & 741 & 2016.204 & 149.1 & 0.2 & 17.247 & 0.059 & 1 & \\
\hline $06558+0246$ & XMI & 30 & 2016.204 & 98.9 & 0.2 & 24.501 & 0.083 & 1 & \\
\hline $06563-0115$ & HJ & 745 & 2016.204 & 274.4 & 0.2 & 10.495 & 0.036 & 1 & \\
\hline $06563+1020$ & BRT & 1218 & 2016.212 & 346.8 & 0.2 & 4.530 & 0.015 & 1 & \\
\hline $06563+1250$ & BRT & 1217 & 2016.212 & 357.9 & 0.2 & 4.261 & 0.014 & 1 & \\
\hline $06567-0147$ & BAL & 364 & 2016.204 & 226.0 & 0.2 & 7.680 & 0.026 & 1 & \\
\hline 06571-0108 & BAL & 743 & 2016.204 & 348.6 & 0.2 & 6.337 & 0.022 & 1 & \\
\hline $06582+0736$ & BRT & 2128 & 2016.212 & 36.7 & 0.2 & 4.425 & 0.015 & 1 & \\
\hline $06584+0649$ & DAN & $269 \mathrm{AB}$ & 2016.212 & 163.9 & 0.2 & 8.413 & 0.029 & 1 & \\
\hline $06587+1159$ & GRV & 726 & 2016.212 & 197.0 & 0.2 & 10.960 & 0.037 & 1 & \\
\hline $06599+0701$ & DOO & 41 & 2016.212 & 323.1 & 0.2 & 5.972 & 0.020 & 1 & \\
\hline $07006+1243$ & STF & $1007 \mathrm{AD}$ & 2016.160 & 28.4 & 0.2 & 67.706 & 0.230 & 1 & \\
\hline $07017+1511$ & $\mathrm{COU}$ & $2706 \mathrm{AD}$ & 2016.212 & 195.5 & 0.2 & 26.174 & 0.089 & 1 & \\
\hline $07017+1511$ & $\mathrm{~J}$ & $1059 \mathrm{AC}$ & 2016.212 & 125.0 & 0.2 & 12.792 & 0.043 & 1 & \\
\hline $07082-0952$ & DAN & 263 & 2016.163 & 31.5 & 0.2 & 10.285 & 0.035 & 1 & \\
\hline $07085+0312$ & BAL & 2248 & 2016.127 & 84.2 & 0.2 & 18.550 & 0.063 & 1 & \\
\hline 07089-0308 & BAL & 134 & 2016.163 & 38.9 & 0.2 & 15.399 & 0.052 & 1 & \\
\hline $07097+0029$ & $\mathrm{~J}$ & 2454 & 2016.163 & 341.9 & 0.2 & 4.811 & 0.016 & 1 & \\
\hline $07098+0526$ & $\mathrm{~J}$ & $702 \mathrm{CE}$ & 2016.128 & 277.9 & 0.2 & 32.003 & 0.109 & 1 & \\
\hline $07100-0044$ & VAS & $11 \mathrm{AB}$ & 2016.163 & 159.0 & 0.2 & 20.814 & 0.071 & 1 & \\
\hline $07100-0044$ & VAS & $11 \mathrm{AC}$ & 2016.163 & 201.3 & 0.2 & 49.201 & 0.167 & 1 & \\
\hline $07113-0918$ & FAL & 20 & 2016.163 & 184.4 & 0.2 & 12.064 & 0.041 & 1 & \\
\hline $07114-0355$ & BRT & 398 & 2016.163 & 142.4 & 0.2 & 4.604 & 0.016 & 1 & \\
\hline $07119+0907$ & XMI & 48 & 2016.128 & 52.7 & 0.2 & 13.034 & 0.044 & 1 & \\
\hline $07127+0645$ & XMI & 49 & 2016.128 & 1.9 & 0.2 & 20.775 & 0.071 & 1 & \\
\hline $07130+0213$ & $\mathrm{~J}$ & 23 & 2016.127 & 282.8 & 0.2 & 5.618 & 0.019 & 1 & \\
\hline $07130+0324$ & FOX & $153 \mathrm{AC}$ & 2016.127 & 49.6 & 0.2 & 22.135 & 0.075 & 1 & \\
\hline $07130+0324$ & HJ & $2365 \mathrm{AB}$ & 2016.127 & 139.3 & 0.2 & 18.383 & 0.063 & 1 & \\
\hline
\end{tabular}


Table 4-Continued

\begin{tabular}{|c|c|c|c|c|c|c|c|c|}
\hline $\begin{array}{l}\text { WDS Desig. } \\
\alpha, \delta(2000)\end{array}$ & $\begin{array}{c}\text { Discoverer } \\
\text { Designation }\end{array}$ & JY & $\begin{array}{c}\theta \\
(\circ)\end{array}$ & $\begin{array}{l}\sigma \theta \\
(\circ)\end{array}$ & $\begin{array}{c}\rho \\
\left({ }^{\prime \prime}\right)\end{array}$ & $\begin{array}{l}\sigma \rho \\
\left({ }^{\prime \prime}\right)\end{array}$ & $\mathrm{n}$ & Note \\
\hline $07134-0008$ & BAL 1088 & 2016.163 & 87.6 & 0.2 & 19.511 & 0.066 & 1 & \\
\hline $07135+1040$ & BRT 1226 & 2016.128 & 26.4 & 0.2 & 4.759 & 0.016 & 1 & \\
\hline $07138+0536$ & XMI & 2016.128 & 172.6 & 0.2 & 20.969 & 0.071 & 1 & \\
\hline $07139+0809$ & XMI 52 & 2016.128 & 336.0 & 0.2 & 20.861 & 0.071 & 1 & \\
\hline $07141-0007$ & BAL 1089 & 2016.163 & 247.1 & 0.2 & 17.248 & 0.059 & 1 & \\
\hline $07143-0224$ & BAL 162 & 2016.163 & 7.6 & 0.2 & 11.479 & 0.039 & 1 & \\
\hline $07143+0923$ & 2456 & 2016.128 & 56.6 & 0.2 & 6.151 & 0.021 & 1 & \\
\hline $07145+0418$ & BAL 2747 & 2016.127 & 56.6 & 0.2 & 16.121 & 0.055 & 1 & \\
\hline $07147+0632$ & BRT 2133 & 2016.128 & 48.1 & 0.2 & 4.538 & 0.015 & 1 & \\
\hline $07150+0956$ & 2457 & 2016.128 & 177.9 & 0.2 & 5.799 & 0.020 & 1 & \\
\hline $07156+0024$ & BAL 1090 & 2016.163 & 221.6 & 0.2 & 15.578 & 0.053 & 1 & \\
\hline $07158+0311$ & BAL 2260 & 2016.114 & 47.4 & 0.2 & 10.345 & 0.035 & 1 & \\
\hline $07161+0126$ & BAL 1396 & 2016.114 & 74.1 & 0.2 & 16.405 & 0.056 & 1 & \\
\hline $07167+0312$ & BAL $2261 \mathrm{AB}$ & 2016.114 & 141.0 & 0.2 & 12.813 & 0.044 & 1 & \\
\hline $07167+0312$ & BAL $2261 \mathrm{AC}$ & 2016.114 & 51.3 & 0.2 & 4.951 & 0.017 & 1 & $\mathrm{~B}$ \\
\hline $07176-0221$ & BAL 167 & 2016.185 & 356.0 & 0.2 & 5.284 & 0.018 & 1 & \\
\hline $07178+0141$ & HJ 2369 & 2016.114 & 57.6 & 0.2 & 18.941 & 0.064 & 1 & \\
\hline $07180+0208$ & BAL 1784 & 2016.114 & 273.3 & 0.2 & 18.649 & 0.063 & 1 & \\
\hline $07182-0020$ & SLE $303 \mathrm{AB}$ & 2016.185 & 81.3 & 0.2 & 22.181 & 0.075 & 1 & \\
\hline $07182-0020$ & SLE $303 \mathrm{AC}$ & 2016.185 & 152.0 & 0.2 & 18.613 & 0.063 & 1 & \\
\hline $07182-0020$ & SLE $303 \mathrm{BC}$ & 2016.185 & 213.6 & 0.2 & 23.697 & 0.081 & 1 & \\
\hline $07189+0233$ & BAL 1786 & 2016.114 & 103.2 & 0.2 & 12.463 & 0.042 & 1 & \\
\hline $07190-0047$ & BAL 792 & 2016.185 & 70.6 & 0.2 & 10.290 & 0.035 & 1 & \\
\hline $07192-0200$ & BAL 469 & 2016.185 & 238.3 & 0.2 & 7.913 & 0.027 & 1 & \\
\hline $07193+0233$ & BAL 1787 & 2016.114 & 85.2 & 0.2 & 8.079 & 0.027 & 1 & \\
\hline $07195-0033$ & BAL 793 & 2016.185 & 71.9 & 0.2 & 15.743 & 0.054 & 1 & \\
\hline $07195+0248$ & BAL 1788 & 2016.114 & 198.1 & 0.2 & 12.459 & 0.042 & 1 & \\
\hline $07196-0303$ & 2810 & 2016.185 & 165.4 & 0.2 & 5.591 & 0.019 & 1 & \\
\hline $07198+0017$ & BAL 1091 & 2016.185 & 36.0 & 0.2 & 16.265 & 0.055 & 1 & \\
\hline $07202+0159$ & BAL 1789 & 2016.114 & 313.8 & 0.2 & 6.323 & 0.021 & 1 & \\
\hline $07203-0132$ & BAL 472 & 2016.185 & 196.8 & 0.2 & 12.872 & 0.044 & 1 & \\
\hline $07204+0117$ & SLE 308 & 2016.114 & 22.6 & 0.2 & 8.049 & 0.027 & 1 & \\
\hline
\end{tabular}


Table 4-Continued

\begin{tabular}{|c|c|c|c|c|c|c|c|c|}
\hline $\begin{array}{l}\text { WDS Desig. } \\
\alpha, \delta(2000)\end{array}$ & $\begin{array}{l}\text { Discoverer } \\
\text { Designation }\end{array}$ & JY & $\begin{array}{c}\theta \\
(0)\end{array}$ & $\begin{array}{l}\sigma \theta \\
(0)\end{array}$ & $\begin{array}{c}\rho \\
\left({ }^{\prime \prime}\right)\end{array}$ & $\begin{array}{l}\sigma \rho \\
\left({ }^{\prime \prime}\right)\end{array}$ & $\mathrm{n}$ & Note \\
\hline $07207+0238$ & BAL 1790 & 2016.114 & 88.8 & 0.2 & 15.759 & 0.054 & 1 & \\
\hline $07208-0129$ & 2811 & 2016.185 & 163.0 & 0.2 & 7.603 & 0.026 & 1 & \\
\hline $07212-0017$ & SLE 309 & 2016.185 & 21.9 & 0.2 & 8.397 & 0.029 & 1 & \\
\hline $07212+0217$ & BAL 1791 & 2016.114 & 178.5 & 0.2 & 9.604 & 0.033 & 1 & \\
\hline $07212+0338$ & BAL 2268 & 2016.114 & 298.3 & 0.2 & 17.803 & 0.061 & 1 & \\
\hline $07214+0027$ & SLE 310 & 2016.185 & 58.5 & 0.2 & 10.501 & 0.036 & 1 & \\
\hline $07222-0056$ & BAL 797 & 2016.185 & 132.1 & 0.2 & 19.507 & 0.066 & 1 & \\
\hline $07225-0215$ & ROE 29 & 2016.185 & 215.3 & 0.2 & 10.689 & 0.036 & 1 & \\
\hline $07234-0358$ & BRT 404 & 2016.185 & 322.4 & 0.2 & 5.113 & 0.017 & 1 & \\
\hline $07236+0023$ & HJ 2378 & 2016.128 & 123.8 & 0.2 & 22.167 & 0.037 & 2 & \\
\hline $07239+0109$ & BAL 1400 & 2016.114 & 321.0 & 0.2 & 16.306 & 0.055 & 1 & \\
\hline $07240+0009$ & SLE 315 & 2016.114 & 210.6 & 0.2 & 9.323 & 0.032 & 1 & \\
\hline $07319-0031$ & STF $1109 \mathrm{AB}$ & 2016.204 & 17.3 & 0.2 & 3.052 & 0.010 & 1 & \\
\hline 07319-0031 & STF 1109 BC & 2016.204 & 299.8 & 0.2 & 25.925 & 0.088 & 1 & \\
\hline $07330-0117$ & BAL 480 & 2016.204 & 323.6 & 0.2 & 19.576 & 0.067 & 1 & \\
\hline $07340-0142$ & $\begin{array}{ll}\mathrm{J} & 1496\end{array}$ & 2016.204 & 9.9 & 0.2 & 11.248 & 0.038 & 1 & \\
\hline $07343-0135$ & 2482 & 2016.204 & 203.5 & 0.2 & 4.442 & 0.015 & 1 & \\
\hline $07351-0108$ & BAL 819 & 2016.204 & 254.8 & 0.2 & 6.077 & 0.021 & 1 & \\
\hline $07360-0147$ & BAL 484 & 2016.204 & 121.4 & 0.2 & 7.272 & 0.025 & 1 & \\
\hline $07367-0101$ & BAL 822 & 2016.204 & 343.4 & 0.2 & 12.553 & 0.043 & 1 & \\
\hline $07376-0140$ & SLE 804 & 2016.204 & 75.1 & 0.2 & 8.693 & 0.030 & 1 & \\
\hline $07385-0312$ & HJ & 2016.038 & 304.7 & 0.2 & 7.027 & 0.024 & 1 & \\
\hline $07388-0126$ & $44 \mathrm{AB}, \mathrm{C}$ & 2016.073 & 264.4 & 0.2 & 17.043 & 0.058 & 1 & \\
\hline $07388-0126$ & $44 \mathrm{AB}, \mathrm{D}$ & 2016.073 & 150.3 & 0.2 & 42.951 & 0.146 & 1 & \\
\hline $07392-0838$ & 1500 & 2016.185 & 180.2 & 0.2 & 6.770 & 0.023 & 1 & \\
\hline 07393-0219 & BAL 184 & 2016.185 & 177.3 & 0.2 & 4.329 & 0.015 & 1 & \\
\hline $07396+0525$ & SLE $\quad 441 \mathrm{AB}$ & 2016.073 & 151.4 & 0.2 & 11.279 & 0.038 & 1 & \\
\hline $07403-0221$ & BAL 187 & 2016.185 & 115.7 & 0.2 & 4.175 & 0.014 & 1 & \\
\hline $07406-0218$ & BAL 186 & 2016.185 & 296.7 & 0.2 & 5.790 & 0.020 & 1 & \\
\hline $07419+0524$ & SLE 447 & 2016.038 & 325.6 & 0.2 & 20.637 & 0.070 & 1 & \\
\hline $07421-0058$ & BAL 824 & 2016.073 & 145.1 & 0.2 & 17.617 & 0.060 & 1 & \\
\hline $07423-0001$ & HJ 2410 & 2016.073 & 350.7 & 0.2 & 17.329 & 0.059 & 1 & \\
\hline
\end{tabular}


Table 4-Continued

\begin{tabular}{|c|c|c|c|c|c|c|c|c|c|}
\hline $\begin{array}{l}\text { WDS Desig. } \\
\alpha, \delta(2000)\end{array}$ & $\begin{array}{l}\text { Dis } \\
\text { Desi }\end{array}$ & $\begin{array}{l}\text { coverer } \\
\text { ignation }\end{array}$ & JY & $\begin{array}{c}\theta \\
(\circ)\end{array}$ & $\begin{array}{l}\sigma \theta \\
(0)\end{array}$ & $\begin{array}{c}\rho \\
\left({ }^{\prime \prime}\right)\end{array}$ & $\begin{array}{l}\sigma \rho \\
\left({ }^{\prime \prime}\right)\end{array}$ & $\mathrm{n}$ & Note \\
\hline $07427+0533$ & SLE & 451 & 2016.073 & 157.4 & 0.2 & 15.642 & 0.053 & 1 & \\
\hline 07430-0319 & $\mathrm{J}$ & 2848 & 2016.185 & 23.9 & 0.2 & 6.244 & 0.021 & 1 & \\
\hline $07437-0004$ & HJ & 2413 & 2016.073 & 8.4 & 0.2 & 11.733 & 0.040 & 1 & \\
\hline $07438-0227$ & J & 2850 & 2016.185 & 238.6 & 0.2 & 6.742 & 0.023 & 1 & \\
\hline $07443-0040$ & BAL & $825 \mathrm{AB}$ & 2016.073 & 157.1 & 0.2 & 6.242 & 0.021 & 1 & \\
\hline $07443-0040$ & BAL & $825 \mathrm{BC}$ & 2016.073 & 287.2 & 0.2 & 17.171 & 0.058 & 1 & B \\
\hline $07448-0123$ & BAL & 487 & 2016.073 & 69.5 & 0.2 & 16.684 & 0.057 & 1 & \\
\hline $07453-0026$ & HJ & $767 \mathrm{AB}$ & 2016.073 & 163.1 & 0.2 & 21.184 & 0.072 & 1 & \\
\hline $07459-0121$ & BAL & 488 & 2016.185 & 69.7 & 0.2 & 16.653 & 0.057 & 1 & \\
\hline $07464+0838$ & TDS & 5186 & 2016.038 & 187.1 & 0.2 & 9.279 & 0.032 & 1 & \\
\hline $07464+1713$ & HJ & 3299 & 2016.185 & 239.7 & 0.2 & 19.341 & 0.066 & 1 & \\
\hline $07478-0125$ & BAL & 489 & 2016.073 & 44.7 & 0.2 & 5.745 & 0.020 & 1 & \\
\hline $07478+1240$ & $\mathrm{~J}$ & $2488 \mathrm{AB}$ & 2016.185 & 116.1 & 0.2 & 56.726 & 0.193 & 1 & \\
\hline $07478+1240$ & $\mathrm{~J}$ & $2488 \mathrm{BC}$ & 2016.185 & 13.4 & 0.2 & 5.237 & 0.018 & 1 & \\
\hline $07487+1436$ & HJ & $3300 \mathrm{AC}$ & 2016.038 & 70.6 & 0.2 & 36.729 & 0.125 & 1 & \\
\hline $07487+1436$ & WSI & $25 \mathrm{AB}$ & 2016.038 & 67.6 & 0.2 & 13.105 & 0.045 & 1 & \\
\hline $07490+1249$ & $\mathrm{~J}$ & 2853 & 2016.185 & 109.3 & 0.2 & 4.738 & 0.016 & 1 & \\
\hline $07491+0946$ & $\mathrm{~J}$ & 2042 & 2016.185 & 39.1 & 0.2 & 4.010 & 0.014 & 1 & \\
\hline 07493-0034 & HJ & 64 & 2016.038 & 325.6 & 0.2 & 13.555 & 0.046 & 1 & \\
\hline 07498-0100 & BAL & 830 & 2016.038 & 180.0 & 0.2 & 9.058 & 0.031 & 1 & \\
\hline $07502-0214$ & GCB & 21 & 2016.038 & 312.7 & 0.2 & 7.381 & 0.025 & 1 & \\
\hline $07504-0336$ & DAM & $346 \mathrm{BC}$ & 2016.169 & 125.6 & 0.2 & 14.957 & 0.051 & 1 & \\
\hline $07506-0205$ & $\mathrm{~J}$ & 2854 & 2016.169 & 69.8 & 0.2 & 6.947 & 0.024 & 1 & \\
\hline $07521+1038$ & $\mathrm{~J}$ & 374 & 2016.169 & 254.4 & 0.2 & 5.534 & 0.019 & 1 & \\
\hline $07522-0659$ & BRT & 414 & 2016.169 & 359.4 & 0.2 & 4.208 & 0.014 & 1 & \\
\hline $07524+0359$ & BAL & 2792 & 2016.169 & 251.1 & 0.2 & 14.998 & 0.051 & 1 & \\
\hline $07524-0707$ & DAM & $347 \mathrm{AC}$ & 2016.169 & 287.5 & 0.2 & 14.971 & 0.051 & 1 & \\
\hline $07527-0703$ & HJ & 2420 & 2016.169 & 341.1 & 0.2 & 11.832 & 0.040 & 1 & \\
\hline $07527+2003$ & $\mathrm{COU}$ & 928 & 2016.832 & 99.0 & 0.2 & 5.505 & 0.019 & 1 & \\
\hline $07528-0355$ & $\mathrm{ROE}$ & 31 & 2016.169 & 86.8 & 0.2 & 8.883 & 0.030 & 1 & \\
\hline $07529-0758$ & $\mathrm{~J}$ & 1504 & 2016.169 & 250.9 & 0.1 & 9.135 & 0.016 & 2 & \\
\hline $07530+0423$ & BAL & 2793 & 2016.169 & 204.6 & 0.2 & 15.174 & 0.052 & 1 & \\
\hline
\end{tabular}


Table 4-Continued

\begin{tabular}{|c|c|c|c|c|c|c|c|c|}
\hline $\begin{array}{l}\text { WDS Desig. } \\
\alpha, \delta(2000)\end{array}$ & $\begin{array}{l}\text { Discoverer } \\
\text { Designation }\end{array}$ & JY & $\begin{array}{c}\theta \\
(\circ)\end{array}$ & $\begin{array}{l}\sigma \theta \\
(\circ)\end{array}$ & $\begin{array}{c}\rho \\
\left({ }^{\prime \prime}\right)\end{array}$ & $\begin{array}{l}\sigma \rho \\
\left({ }^{\prime \prime}\right)\end{array}$ & $\mathrm{n}$ & Note \\
\hline $07530-0805$ & 419 & 2016.169 & 319.0 & 0.2 & 4.243 & 0.014 & 1 & \\
\hline $07532+1130$ & BRT 1242 & 2016.169 & 239.4 & 0.2 & 3.834 & 0.013 & 1 & \\
\hline $07535+0348$ & BAL 2795 & 2016.169 & 134.8 & 0.2 & 10.990 & 0.037 & 1 & \\
\hline $07536+0435$ & BAL 2796 & 2016.169 & 328.3 & 0.2 & 16.985 & 0.058 & 1 & \\
\hline $07548+1118$ & HJ & 2016.169 & 231.1 & 0.2 & 26.580 & 0.090 & 1 & \\
\hline $07558+0202$ & BAL 1827 & 2016.169 & 340.2 & 0.2 & 7.597 & 0.026 & 1 & \\
\hline $07563+0150$ & BAL 1828 & 2016.169 & 332.8 & 0.2 & 15.428 & 0.052 & 1 & \\
\hline $07565+2152$ & STF $1158 \mathrm{AB}$ & 2016.832 & 334.0 & 0.2 & 7.554 & 0.026 & 1 & \\
\hline $07565+2152$ & STF $1158 \mathrm{AC}$ & 2016.832 & 246.1 & 0.2 & 21.037 & 0.072 & 1 & \\
\hline $07572+2342$ & 433 & 2016.832 & 8.5 & 0.2 & 14.736 & 0.050 & 1 & \\
\hline $07580+2104$ & 434 & 2016.832 & 103.5 & 0.2 & 22.972 & 0.078 & 1 & \\
\hline $07592+0413$ & HJ & 2016.169 & 357.0 & 0.2 & 22.855 & 0.078 & 1 & \\
\hline $08009+0334$ & BAL 2318 & 2016.210 & 284.1 & 0.2 & 14.966 & 0.051 & 1 & \\
\hline $08010+3653$ & 3305 & 2016.210 & 226.4 & 0.2 & 4.617 & 0.016 & 1 & \\
\hline $08018+1154$ & SLE & 2016.169 & 308.5 & 0.2 & 19.971 & 0.068 & 1 & \\
\hline $08025+0305$ & $23 \mathrm{CD}$ & 2016.169 & 235.0 & 0.2 & 11.145 & 0.038 & 1 & \\
\hline $08025-0947$ & BRT 416 & 2016.169 & 135.3 & 0.2 & 3.688 & 0.013 & 1 & \\
\hline $08025+6305$ & $86 \mathrm{AB}$ & 2016.210 & 80.4 & 0.2 & 51.265 & 0.174 & 1 & \\
\hline $08028+1203$ & SLE 457 & 2016.210 & 182.4 & 0.2 & 27.617 & 0.094 & 1 & \\
\hline $08037+0356$ & BAL 2802 & 2016.158 & 274.3 & 0.2 & 11.190 & 0.038 & 1 & \\
\hline $08056+0410$ & BAL 2805 & 2016.158 & 85.8 & 0.2 & 17.307 & 0.059 & 1 & \\
\hline $08056+2732$ & STF 1177 & 2016.832 & 349.7 & 0.2 & 3.539 & 0.012 & 1 & \\
\hline $08060-0614$ & 2868 & 2016.158 & 6.9 & 0.2 & 6.473 & 0.022 & 1 & \\
\hline $08074+0012$ & BAL 1132 & 2016.158 & 264.2 & 0.2 & 6.971 & 0.024 & 1 & \\
\hline $08077-0259$ & HJ & 2016.158 & 296.6 & 0.2 & 18.715 & 0.064 & 1 & \\
\hline $08078+0222$ & BAL 1836 & 2016.158 & 162.1 & 0.2 & 5.950 & 0.020 & 1 & \\
\hline $08084-0102$ & BAL 846 & 2016.158 & 78.2 & 0.2 & 19.510 & 0.066 & 1 & \\
\hline $08086-0205$ & XMI & 2016.158 & 191.0 & 0.2 & 27.319 & 0.093 & 1 & \\
\hline $08100+2329$ & 440 & 2016.832 & 123.1 & 0.2 & 15.777 & 0.054 & 1 & \\
\hline $08102+0240$ & BAL 1837 & 2005.159 & 230.9 & 0.2 & 4.100 & 0.014 & 1 & A \\
\hline $08104+0020$ & BAL 1133 & 2016.158 & 315.7 & 0.2 & 6.605 & 0.022 & 1 & \\
\hline $08112+3527$ & POP 133 & 2016.212 & 33.7 & 0.2 & 9.627 & 0.033 & 1 & \\
\hline
\end{tabular}


Table 4-Continued

\begin{tabular}{|c|c|c|c|c|c|c|c|c|c|}
\hline $\begin{array}{l}\text { WDS Desig. } \\
\alpha, \delta(2000)\end{array}$ & & $\begin{array}{l}\text { scoverer } \\
\text { signation }\end{array}$ & JY & $\begin{array}{c}\theta \\
(0)\end{array}$ & $\begin{array}{l}\sigma \theta \\
(0)\end{array}$ & $\begin{array}{c}\rho \\
\left({ }^{\prime \prime}\right)\end{array}$ & $\begin{array}{l}\sigma \rho \\
\left({ }^{\prime \prime}\right)\end{array}$ & $\mathrm{n}$ & Note \\
\hline $08117-0636$ & $\mathrm{~J}$ & 2054 & 2016.158 & 132.4 & 0.2 & 7.214 & 0.025 & 1 & \\
\hline $08122+1739$ & STF & $1196 \mathrm{AC}$ & 2016.832 & 61.5 & 0.2 & 6.247 & 0.021 & 1 & \\
\hline $08122+1739$ & STF & $1196 \mathrm{BC}$ & 2016.832 & 69.3 & 0.2 & 5.582 & 0.019 & 1 & \\
\hline $08123+0157$ & BAL & 1839 & 2016.158 & 120.3 & 0.2 & 13.991 & 0.048 & 1 & \\
\hline $08135-0123$ & HJ & 85 & 2016.158 & 84.5 & 0.2 & 16.553 & 0.056 & 1 & \\
\hline 08136-0159 & HJ & 778 & 2016.158 & 139.1 & 0.2 & 4.774 & 0.016 & 1 & \\
\hline $08143-0149$ & BAL & 499 & 2016.158 & 126.7 & 0.2 & 16.103 & 0.055 & 1 & \\
\hline $08147+0432$ & BAL & 2808 & 2016.158 & 117.3 & 0.2 & 13.983 & 0.048 & 1 & \\
\hline $08147+0438$ & WSI & 162 & 2016.158 & 249.6 & 0.2 & 20.563 & 0.070 & 1 & B \\
\hline $08163+3423$ & GRV & 755 & 2016.212 & 155.0 & 0.2 & 40.673 & 0.138 & 1 & \\
\hline 08169-0016 & BAL & 1135 & 2016.158 & 92.7 & 0.2 & 10.650 & 0.036 & 1 & \\
\hline $08172+2623$ & HJ & 781 & 2016.832 & 323.8 & 0.2 & 6.068 & 0.021 & 1 & \\
\hline $08173-0058$ & $\mathrm{~J}$ & 422 & 2016.158 & 71.9 & 0.2 & 4.898 & 0.017 & 1 & \\
\hline $08174+0312$ & BAL & 2327 & 2016.163 & 337.0 & 0.2 & 7.341 & 0.025 & 1 & \\
\hline $08177-0015$ & BAL & 1136 & 2016.163 & 4.6 & 0.2 & 18.230 & 0.062 & 1 & \\
\hline $08181+0436$ & BAL & 2811 & 2016.163 & 272.1 & 0.2 & 14.802 & 0.050 & 1 & \\
\hline $08184-2017$ & HJ & $2441 \mathrm{AB}$ & 2016.996 & 141.5 & 0.2 & 13.818 & 0.047 & 1 & \\
\hline $08184-2017$ & HJ & $2441 \mathrm{AC}$ & 2016.996 & 152.7 & 0.2 & 54.222 & 0.184 & 1 & \\
\hline $08208+0318$ & BAL & 2329 & 2016.163 & 302.2 & 0.2 & 19.606 & 0.067 & 1 & \\
\hline $08222+0144$ & BAL & 1843 & 2016.163 & 329.6 & 0.2 & 17.200 & 0.058 & 1 & \\
\hline $08236-1920$ & GWP & 1011 & 2016.996 & 315.7 & 0.2 & 35.227 & 0.120 & 1 & \\
\hline $08253+2421$ & STF & 1220 & 2016.832 & 215.2 & 0.2 & 29.242 & 0.099 & 1 & \\
\hline $08258+0557$ & SCJ & 9 & 2016.832 & 332.1 & 0.2 & 3.794 & 0.013 & 1 & \\
\hline 08267-1910 & FEN & 14 & 2016.996 & 309.8 & 0.2 & 4.375 & 0.015 & 1 & \\
\hline $08267+2432$ & STF & $1224 \mathrm{~A}, \mathrm{BC}$ & 2016.832 & 51.7 & 0.2 & 5.717 & 0.019 & 1 & \\
\hline $08268+2656$ & $\mathrm{STF}$ & 1223 & 2016.832 & 218.3 & 0.2 & 5.229 & 0.018 & 1 & \\
\hline $08275+0426$ & HJ & 92 & 2016.163 & 57.7 & 0.2 & 24.701 & 0.084 & 1 & \\
\hline $08306+0007$ & BAL & 1138 & 2016.163 & 7.0 & 0.2 & 5.610 & 0.019 & 1 & \\
\hline $08358+0637$ & STF & $1245 \mathrm{AB}$ & 2016.832 & 24.8 & 0.2 & 10.044 & 0.034 & 1 & \\
\hline $08360-0007$ & BAL & 1139 & 2016.158 & 268.8 & 0.2 & 18.820 & 0.064 & 1 & \\
\hline $08380-0141$ & BAL & 509 & 2016.158 & 115.7 & 0.2 & 18.829 & 0.064 & 1 & \\
\hline $08399+1933$ & $\mathrm{~S}$ & $571 \mathrm{AC}$ & 2016.832 & 156.5 & 0.2 & 45.398 & 0.154 & 1 & \\
\hline
\end{tabular}


Table 4-Continued

\begin{tabular}{|c|c|c|c|c|c|c|c|c|c|}
\hline $\begin{array}{l}\text { WDS Desig. } \\
\alpha, \delta(2000)\end{array}$ & \multicolumn{2}{|c|}{$\begin{array}{c}\text { Discoverer } \\
\text { Designation }\end{array}$} & JY & $\begin{array}{c}\theta \\
(\circ)\end{array}$ & $\begin{array}{l}\sigma \theta \\
(\circ)\end{array}$ & $\begin{array}{c}\rho \\
\left({ }^{\prime \prime}\right)\end{array}$ & $\begin{array}{l}\sigma \rho \\
\left({ }^{\prime \prime}\right)\end{array}$ & $\mathrm{n}$ & Note \\
\hline $08401-0115$ & $\mathrm{~J}$ & 423 & 2016.158 & 283.9 & 0.2 & 6.899 & 0.023 & 1 & \\
\hline $08405-0239$ & $\mathrm{~J}$ & 3289 & 2016.158 & 94.9 & 0.2 & 4.236 & 0.014 & 1 & \\
\hline $08405-2049$ & ARA & 1059 & 2016.996 & 90.2 & 0.2 & 9.930 & 0.034 & 1 & \\
\hline $08407+3508$ & HJ & 793 & 2016.210 & 252.0 & 0.2 & 11.047 & 0.038 & 1 & \\
\hline $08423-0824$ & STF & 1264 & 2016.996 & 269.8 & 0.2 & 6.433 & 0.022 & 1 & \\
\hline $08436-0237$ & $\mathrm{~J}$ & 1530 & 2016.158 & 302.6 & 0.2 & 12.487 & 0.042 & 1 & \\
\hline $08444+3029$ & HJ & 455 & 2016.212 & 344.8 & 0.2 & 10.899 & 0.037 & 1 & \\
\hline $08453-0109$ & BAL & 856 & 2016.158 & 22.8 & 0.2 & 18.524 & 0.063 & 1 & \\
\hline $08453-0236$ & STF & 1270 & 2016.832 & 264.8 & 0.2 & 4.669 & 0.016 & 1 & \\
\hline $08484-1641$ & $\mathrm{POC}$ & 2 & 2016.996 & 75.2 & 0.2 & 6.138 & 0.021 & 1 & \\
\hline $08494-1711$ & $\mathrm{POC}$ & 3 & 2016.996 & 228.0 & 0.2 & 7.752 & 0.026 & 1 & \\
\hline $08512-1231$ & XMI & 84 & 2016.996 & 268.3 & 0.2 & 20.984 & 0.071 & 1 & \\
\hline $08513-0958$ & $\mathrm{~J}$ & 2648 & 2016.996 & 354.6 & 0.2 & 8.441 & 0.029 & 1 & \\
\hline $08532+4625$ & TOB & 49 & 2016.212 & 254.3 & 0.2 & 20.421 & 0.069 & 1 & \\
\hline $08542+3035$ & STF & $1291 \mathrm{AB}$ & 2016.832 & 309.5 & 0.2 & 1.563 & 0.005 & 1 & \\
\hline $08555-0758$ & STF & 1295 & 2016.832 & 4.0 & 0.2 & 4.051 & 0.014 & 1 & \\
\hline $08562-0359$ & $\mathrm{~J}$ & 1536 & 2016.114 & 73.6 & 0.2 & 7.801 & 0.027 & 1 & \\
\hline $08568-2009$ & ARA & 648 & 2016.996 & 351.5 & 0.2 & 10.743 & 0.037 & 1 & \\
\hline $08584-0549$ & HDS & 1299 & 2016.163 & 346.6 & 0.2 & 12.003 & 0.041 & 1 & \\
\hline $09003+5332$ & $\mathrm{CBL}$ & 33 & 2016.212 & 113.9 & 0.2 & 29.467 & 0.100 & 1 & \\
\hline $09074+2259$ & STF & $1311 \mathrm{AB}$ & 2016.163 & 198.8 & 0.2 & 7.628 & 0.026 & 1 & \\
\hline 09079-0708 & STF & $1316 \mathrm{AB}$ & 2016.172 & 136.1 & 0.2 & 7.294 & 0.037 & 2 & \\
\hline $09125+4912$ & GRF & $29 \mathrm{AC}$ & 2016.158 & 53.9 & 0.2 & 22.446 & 0.076 & 1 & \\
\hline $09134+5133$ & ES & 716 & 2016.158 & 295.6 & 0.2 & 7.351 & 0.025 & 1 & \\
\hline $09185-0733$ & $\mathrm{~J}$ & 2651 & 2016.163 & 209.5 & 0.2 & 3.899 & 0.013 & 1 & \\
\hline $09234+5136$ & ES & 718 & 2016.158 & 28.1 & 0.2 & 7.839 & 0.027 & 1 & \\
\hline $09256+5401$ & STF & $1346 \mathrm{AB}$ & 2016.213 & 314.1 & 0.2 & 5.740 & 0.020 & 1 & \\
\hline $09265-0255$ & $\mathrm{~J}$ & $425 \mathrm{AC}$ & 2016.114 & 267.3 & 0.2 & 6.291 & 0.021 & 1 & \\
\hline $09354+3958$ & STF & $1369 \mathrm{AB}$ & 2016.208 & 149.6 & 0.0 & 24.887 & 0.016 & 2 & \\
\hline $09355+4006$ & $\mathrm{CBL}$ & 36 & 2016.213 & 132.0 & 0.2 & 13.248 & 0.045 & 1 & \\
\hline $09419+3434$ & STF & 1375 & 2016.213 & 305.7 & 0.2 & 7.221 & 0.025 & 1 & \\
\hline $09508+3138$ & STF & $1383 \mathrm{AB}$ & 2016.213 & 227.6 & 0.2 & 22.484 & 0.076 & 1 & \\
\hline
\end{tabular}


Table 4-Continued

\begin{tabular}{|c|c|c|c|c|c|c|c|c|c|}
\hline \multirow{2}{*}{$\begin{array}{c}\text { WDS Desig. } \\
\alpha, \delta(2000) \\
09541+0457\end{array}$} & \multicolumn{2}{|c|}{$\begin{array}{l}\text { Discoverer } \\
\text { Designation }\end{array}$} & JY & $\begin{array}{c}\theta \\
(0)\end{array}$ & $\begin{array}{l}\sigma \theta \\
(\circ)\end{array}$ & $\begin{array}{c}\rho \\
\left({ }^{\prime \prime}\right)\end{array}$ & $\begin{array}{l}\sigma \rho \\
\left({ }^{\prime \prime}\right)\end{array}$ & $\mathrm{n}$ & Note \\
\hline & $\mathrm{S}$ & 605 & 2016.193 & 286.7 & 0.0 & 53.451 & 0.027 & 3 & \\
\hline $10040-1806$ & SHJ & $110 \mathrm{AC}$ & 2016.210 & 273.7 & 0.0 & 21.151 & 0.019 & 2 & \\
\hline $10092+3222$ & STF & 1411 & 2016.213 & 309.9 & 0.2 & 31.491 & 0.107 & 1 & \\
\hline $10167+5737$ & HJ & $1176 \mathrm{AB}$ & 2016.213 & 317.7 & 0.2 & 8.942 & 0.030 & 1 & \\
\hline $10178+7104$ & STF & $1415 \mathrm{AB}$ & 2016.213 & 167.6 & 0.2 & 16.613 & 0.056 & 1 & \\
\hline $10304+5621$ & STF & 1436 & 2016.213 & 254.2 & 0.2 & 10.489 & 0.036 & 1 & \\
\hline $10385+0235$ & GRV & 819 & 2016.114 & 82.5 & 0.2 & 25.544 & 0.087 & 1 & \\
\hline $10385+0523$ & HJ & 835 & 2016.114 & 9.3 & 0.2 & 15.742 & 0.054 & 1 & \\
\hline $10410+0336$ & $\mathrm{~J}$ & 1369 & 2016.114 & 120.0 & 0.2 & 7.341 & 0.025 & 1 & \\
\hline $10427+4812$ & ES & 603 & 2016.158 & 101.6 & 0.2 & 13.400 & 0.046 & 1 & \\
\hline 10429-0006 & BAL & 1155 & 2016.114 & 202.7 & 0.2 & 17.640 & 0.060 & 1 & \\
\hline $10433+0445$ & STF & $1466 \mathrm{AB}$ & 2016.163 & 239.7 & 0.2 & 6.795 & 0.023 & 1 & \\
\hline $10440+0525$ & SLE & 590 & 2016.114 & 164.2 & 0.2 & 12.862 & 0.044 & 1 & \\
\hline $10459+0426$ & BAL & 2844 & 2016.114 & 329.0 & 0.2 & 4.951 & 0.017 & 1 & \\
\hline $10473+0445$ & SLE & 593 & 2016.114 & 334.5 & 0.2 & 6.379 & 0.022 & 1 & \\
\hline $10483+5500$ & STI & 2255 & 2016.158 & 59.9 & 0.2 & 11.849 & 0.040 & 1 & \\
\hline $10497+0343$ & BAL & 2845 & 2016.114 & 158.9 & 0.2 & 5.717 & 0.019 & 1 & \\
\hline $10521+4804$ & HJ & 2546 & 2016.158 & 56.2 & 0.2 & 6.243 & 0.021 & 1 & \\
\hline $10561+0418$ & GRV & 825 & 2016.114 & 308.2 & 0.2 & 30.613 & 0.104 & 1 & \\
\hline $10598-0200$ & BAL & 530 & 2016.158 & 252.7 & 0.2 & 10.767 & 0.037 & 1 & \\
\hline $11010+2842$ & BRT & 25 & 2016.158 & 3.8 & 0.2 & 4.090 & 0.014 & 1 & \\
\hline $11022-0335$ & BRT & 548 & 2016.114 & 89.3 & 0.2 & 4.173 & 0.014 & 1 & \\
\hline $11041-0031$ & BAL & 1156 & 2016.158 & 94.8 & 0.2 & 9.263 & 0.031 & 1 & \\
\hline 11070-0046 & BAL & 864 & 2016.158 & 324.8 & 0.2 & 4.111 & 0.014 & 1 & \\
\hline $11073+2832$ & HJ & 1185 & 2016.158 & 13.8 & 0.2 & 16.186 & 0.055 & 1 & \\
\hline $11074-0114$ & BAL & 865 & 2016.114 & 217.6 & 0.2 & 9.554 & 0.032 & 1 & \\
\hline $11079+3604$ & KZA & 1 & 2016.185 & 178.6 & 0.2 & 8.019 & 0.027 & 1 & \\
\hline $11095-0325$ & HJ & 177 & 2016.114 & 131.3 & 0.2 & 4.807 & 0.016 & 1 & \\
\hline $11185+0025$ & BAL & 1158 & 2016.158 & 45.2 & 0.2 & 5.559 & 0.019 & 1 & \\
\hline $11254+2428$ & $\mathrm{POU}$ & $3102 \mathrm{AB}$ & 2016.158 & 240.0 & 0.2 & 5.966 & 0.020 & 1 & \\
\hline $11291+3920$ & STF & $1543 \mathrm{EF}$ & 2016.169 & 129.2 & 0.2 & 43.735 & 0.149 & 1 & \\
\hline 11304-0316 & GWP & 1595 & 2016.185 & 212.0 & 0.2 & 53.649 & 0.182 & 1 & \\
\hline
\end{tabular}


Table 4-Continued

\begin{tabular}{|c|c|c|c|c|c|c|c|c|c|}
\hline $\begin{array}{l}\text { WDS Desig. } \\
\alpha, \delta(2000)\end{array}$ & & $\begin{array}{l}\text { iscoverer } \\
\text { signation }\end{array}$ & JY & $\begin{array}{l}\theta \\
\text { (o) }\end{array}$ & $\begin{array}{l}\sigma \theta \\
(0)\end{array}$ & $\begin{array}{c}\rho \\
\left({ }^{\prime \prime}\right)\end{array}$ & $\begin{array}{l}\sigma \rho \\
\left({ }^{\prime \prime}\right)\end{array}$ & $\mathrm{n}$ & Note \\
\hline $11324+2710$ & SLE & 606 & 2016.169 & 84.8 & 0.2 & 10.568 & 0.036 & 1 & \\
\hline $11346+4052$ & $\mathrm{ES}$ & 1401 & 2016.169 & 327.1 & 0.2 & 6.332 & 0.022 & 1 & \\
\hline $11350+5557$ & STI & 2273 & 2016.169 & 298.0 & 0.2 & 5.163 & 0.018 & 1 & \\
\hline $11440+0721$ & $\mathrm{~J}$ & 1016 & 2016.169 & 185.0 & 0.2 & 3.793 & 0.013 & 1 & \\
\hline $11466+5710$ & STI & 2276 & 2016.169 & 8.8 & 0.2 & 11.358 & 0.039 & 1 & \\
\hline $11503+3741$ & HJ & $510 \mathrm{AB}, \mathrm{C}$ & 2016.169 & 252.9 & 0.2 & 24.998 & 0.085 & 1 & \\
\hline $11512+4405$ & STF & 1574 & 2016.185 & 6.2 & 0.2 & 9.403 & 0.032 & 1 & \\
\hline $11525-0245$ & $\mathrm{~J}$ & 1582 & 2016.185 & 166.1 & 0.2 & 10.678 & 0.036 & 1 & \\
\hline $12032+0044$ & BAL & 1450 & 2016.185 & 207.4 & 0.2 & 22.666 & 0.077 & 1 & \\
\hline $12033-0123$ & BAL & 866 & 2016.185 & 353.3 & 0.2 & 9.054 & 0.031 & 1 & \\
\hline $12036+3304$ & $\mathrm{KU}$ & $101 \mathrm{AB}$ & 2016.185 & 51.6 & 0.2 & 53.703 & 0.183 & 1 & \\
\hline $12056+5659$ & $\mathrm{~A}$ & $1358 \mathrm{AB}, \mathrm{C}$ & 2016.185 & 174.0 & 0.2 & 34.775 & 0.118 & 1 & \\
\hline $12083-0037$ & $\mathrm{UC}$ & 2279 & 2016.185 & 245.0 & 0.2 & 48.875 & 0.166 & 1 & \\
\hline $12109+6724$ & HJ & 3336 & 2016.185 & 293.8 & 0.2 & 9.061 & 0.031 & 1 & \\
\hline $12113+3316$ & HJ & 2600 & 2016.185 & 342.1 & 0.2 & 11.745 & 0.040 & 1 & \\
\hline $12151+8349$ & WFC & 124 & 2016.204 & 133.5 & 0.2 & 4.542 & 0.015 & 1 & \\
\hline $12153+6359$ & MLB & 410 & 2016.204 & 34.2 & 0.2 & 6.626 & 0.023 & 1 & \\
\hline $12281+5949$ & $\mathrm{UC}$ & $172 \mathrm{AB}$ & 2016.204 & 120.8 & 0.2 & 5.936 & 0.020 & 1 & \\
\hline $12281+5949$ & $\mathrm{UC}$ & $172 \mathrm{AC}$ & 2016.204 & 221.1 & 0.2 & 45.517 & 0.155 & 1 & \\
\hline $12300+7324$ & HJ & 2613 & 2016.204 & 307.0 & 0.2 & 24.879 & 0.085 & 1 & \\
\hline $12307+7518$ & DOO & 55 & 2016.204 & 54.2 & 0.2 & 4.335 & 0.015 & 1 & \\
\hline $12351+1823$ & STF & 1657 & 2016.431 & 271.5 & 0.0 & 20.373 & 0.005 & 2 & \\
\hline $13256+2116$ & HJ & 2651 & 2016.451 & 339.9 & 0.2 & 13.829 & 0.047 & 1 & \\
\hline $13264+0854$ & WS & 9002 & 2016.451 & 139.9 & 0.2 & 18.973 & 0.065 & 1 & \\
\hline $13311+1216$ & $\mathrm{UC}$ & 2550 & 2016.451 & 292.9 & 0.2 & 10.603 & 0.036 & 1 & \\
\hline $13333+2301$ & STF & 1756 & 2016.451 & 178.2 & 0.2 & 14.584 & 0.050 & 1 & \\
\hline $13552+0128$ & GRV & 875 & 2016.451 & 93.7 & 0.2 & 27.569 & 0.094 & 1 & \\
\hline $14009+0912$ & HEI & 777 & 2005.354 & 214.0 & 0.2 & 1.803 & 0.006 & 1 & A \\
\hline $14150+3545$ & $\mathrm{UC}$ & 2713 & 2016.434 & 206.5 & 0.2 & 42.568 & 0.145 & 1 & \\
\hline $14185+3837$ & HJ & 545 & 2016.434 & 243.7 & 0.2 & 9.168 & 0.031 & 1 & \\
\hline $14201+5402$ & HJ & 2712 & 2016.412 & 297.7 & 0.2 & 28.315 & 0.096 & 1 & \\
\hline $14215+5354$ & STF & 1839 & 2016.412 & 83.0 & 0.2 & 14.544 & 0.049 & 1 & \\
\hline
\end{tabular}


Table 4-Continued

\begin{tabular}{|c|c|c|c|c|c|c|c|c|c|}
\hline $\begin{array}{l}\text { WDS Desig. } \\
\alpha, \delta(2000)\end{array}$ & $\begin{array}{l}\text { Dis } \\
\text { Des }\end{array}$ & $\begin{array}{l}\text { scoverer } \\
\text { signation }\end{array}$ & JY & $\begin{array}{c}\theta \\
(0)\end{array}$ & $\begin{array}{l}\sigma \theta \\
(0)\end{array}$ & $\begin{array}{l}\rho \\
\left({ }^{\prime \prime}\right)\end{array}$ & $\begin{array}{l}\sigma \rho \\
\left({ }^{\prime \prime}\right)\end{array}$ & $\mathrm{n}$ & Note \\
\hline $14222+4832$ & $\mathrm{BU}$ & 615 & 2016.412 & 236.2 & 0.2 & 2.969 & 0.010 & 1 & \\
\hline $14270+3517$ & HJ & $550 \mathrm{AB}$ & 2016.434 & 111.2 & 0.2 & 31.928 & 0.109 & 1 & \\
\hline $14286+4932$ & BEM & 11 & 2016.412 & 17.4 & 0.2 & 14.791 & 0.050 & 1 & \\
\hline $14305+5013$ & BEM & 12 & 2016.412 & 187.1 & 0.2 & 8.858 & 0.030 & 1 & \\
\hline $14312+4807$ & $\mathrm{ES}$ & 961 & 2016.412 & 320.2 & 0.2 & 6.609 & 0.022 & 1 & \\
\hline $14316+5016$ & BEM & 13 & 2016.412 & 131.6 & 0.2 & 29.342 & 0.100 & 1 & \\
\hline $14326+3522$ & ALI & 130 & 2016.434 & 183.4 & 0.2 & 8.206 & 0.028 & 1 & \\
\hline $14338+4707$ & BEM & 9004 & 2016.412 & 317.7 & 0.2 & 23.136 & 0.079 & 1 & \\
\hline $14375+4743$ & $\mathrm{ES}$ & $609 \mathrm{AB}$ & 2016.412 & 15.7 & 0.2 & 4.833 & 0.016 & 1 & \\
\hline $14375+4743$ & $\mathrm{ES}$ & $609 \mathrm{AC}$ & 2016.412 & 118.8 & 0.2 & 79.225 & 0.269 & 1 & \\
\hline $14384+3632$ & ALI & 367 & 2016.434 & 273.3 & 0.2 & 14.548 & 0.049 & 1 & \\
\hline $14407+4928$ & BEM & 9013 & 2016.412 & 332.5 & 0.2 & 5.287 & 0.018 & 1 & \\
\hline $14431+2847$ & HJ & 5487 & 2016.451 & 240.1 & 0.2 & 19.575 & 0.067 & 1 & \\
\hline $14471+0058$ & STF & 1881 & 2016.412 & 2.5 & 0.2 & 3.502 & 0.012 & 1 & \\
\hline $14484+2422$ & STF & 1884 & 2016.467 & 56.5 & 0.2 & 2.196 & 0.007 & 1 & \\
\hline $14501-0710$ & $\mathrm{HO}$ & 546 & 2016.412 & 90.9 & 0.2 & 3.644 & 0.012 & 1 & \\
\hline $14559+2919$ & GRV & 895 & 2016.451 & 40.1 & 0.2 & 11.070 & 0.038 & 1 & \\
\hline $15092+5257$ & BEM & 9020 & 2016.434 & 5.8 & 0.2 & 13.451 & 0.046 & 1 & \\
\hline $15097+4942$ & BEM & 16 & 2016.434 & 67.4 & 0.2 & 25.389 & 0.086 & 1 & \\
\hline $15128+5617$ & STI & 2318 & 2016.451 & 138.0 & 0.2 & 8.989 & 0.031 & 1 & \\
\hline $15149+5048$ & $\mathrm{ES}$ & 775 & 2016.434 & 220.7 & 0.2 & 6.655 & 0.023 & 1 & \\
\hline $15156+5527$ & WZ & 13 & 2016.451 & 16.5 & 0.2 & 9.111 & 0.031 & 1 & \\
\hline $15198+5245$ & $\mathrm{ES}$ & $741 \mathrm{AB}$ & 2016.434 & 235.1 & 0.2 & 8.912 & 0.030 & 1 & \\
\hline $15200+4603$ & $\mathrm{ES}$ & $75 \mathrm{AB}, \mathrm{C}$ & 2016.434 & 36.0 & 0.2 & 4.426 & 0.015 & 1 & \\
\hline $15266+5454$ & STF & 1948 & 2016.451 & 48.1 & 0.2 & 12.855 & 0.044 & 1 & \\
\hline $15269+4610$ & $\mathrm{KU}$ & 50 & 2016.434 & 337.3 & 0.2 & 2.939 & 0.010 & 1 & \\
\hline $15271+5127$ & TDS & 9521 & 2016.434 & 138.3 & 0.2 & 3.306 & 0.011 & 1 & \\
\hline $15292+8027$ & STF & $1972 \mathrm{AB}$ & 2016.451 & 80.5 & 0.2 & 31.848 & 0.108 & 1 & \\
\hline $15370+5824$ & MLB & 71 & 2016.434 & 290.1 & 0.2 & 4.069 & 0.014 & 1 & \\
\hline $15372+6156$ & STI & 786 & 2016.434 & 268.3 & 0.2 & 5.844 & 0.020 & 1 & \\
\hline $15382+3615$ & STF & $1964 \mathrm{AC}$ & 2016.451 & 86.8 & 0.2 & 15.074 & 0.051 & 1 & \\
\hline $15385+4112$ & KZA & 107 & 2016.451 & 327.1 & 0.2 & 29.003 & 0.099 & 1 & \\
\hline
\end{tabular}


Table 4-Continued

\begin{tabular}{|c|c|c|c|c|c|c|c|c|c|}
\hline $\begin{array}{l}\text { WDS Desig. } \\
\alpha, \delta(2000)\end{array}$ & & $\begin{array}{l}\text { iscoverer } \\
\text { signation }\end{array}$ & JY & $\begin{array}{l}\theta \\
(0)\end{array}$ & $\begin{array}{l}\sigma \theta \\
(0)\end{array}$ & $\begin{array}{c}\rho \\
\left({ }^{\prime \prime}\right)\end{array}$ & $\begin{array}{l}\sigma \rho \\
\left({ }^{\prime \prime}\right)\end{array}$ & $\mathrm{n}$ & Note \\
\hline $15392+2330$ & $\mathrm{POU}$ & 3197 & 2016.434 & 352.6 & 0.2 & 4.036 & 0.014 & 1 & \\
\hline $15394+3638$ & STF & 1965 & 2016.451 & 307.0 & 0.2 & 6.428 & 0.022 & 1 & \\
\hline $15416+5351$ & MMA & 35 & 2016.467 & 8.9 & 0.2 & 31.149 & 0.106 & 1 & \\
\hline $15449+5926$ & STF & $1976 \mathrm{AB}$ & 2016.467 & 71.7 & 0.2 & 19.293 & 0.066 & 1 & \\
\hline $15456+2412$ & $\mathrm{POU}$ & 3203 & 2016.434 & 107.4 & 0.2 & 16.713 & 0.057 & 1 & \\
\hline $15456+5342$ & FOX & 191 & 2016.467 & 127.5 & 0.2 & 5.952 & 0.020 & 1 & \\
\hline $15469+4736$ & ES & 1087 & 2016.467 & 174.6 & 0.2 & 6.832 & 0.023 & 1 & \\
\hline $15470+5159$ & $\mathrm{HU}$ & 152 & 2016.467 & 244.0 & 0.2 & 3.863 & 0.013 & 1 & \\
\hline $15485+4037$ & HJ & 573 & 2016.451 & 51.1 & 0.2 & 14.699 & 0.050 & 1 & \\
\hline $15496+2244$ & $\mathrm{POU}$ & 3205 & 2016.434 & 334.6 & 0.2 & 8.274 & 0.028 & 1 & \\
\hline $15498+4349$ & ES & 1554 & 2016.467 & 3.6 & 0.2 & 4.927 & 0.017 & 1 & \\
\hline $15509+2322$ & $\mathrm{POU}$ & 3209 & 2016.434 & 48.4 & 0.2 & 13.982 & 0.048 & 1 & \\
\hline $15511+5254$ & STF & $1984 \mathrm{AB}$ & 2016.451 & 280.4 & 0.2 & 6.392 & 0.022 & 1 & \\
\hline $15517+4614$ & BEM & 9006 & 2016.467 & 258.8 & 0.2 & 5.143 & 0.017 & 1 & \\
\hline $15518+7319$ & $\mathrm{UC}$ & 3072 & 2016.467 & 40.1 & 0.2 & 42.123 & 0.143 & 1 & \\
\hline $15520+5234$ & $\mathrm{AG}$ & 199 & 2016.451 & 253.5 & 0.2 & 9.588 & 0.033 & 1 & \\
\hline $15529+6205$ & STI & 788 & 2016.467 & 50.1 & 0.2 & 11.618 & 0.039 & 1 & \\
\hline $15574+6833$ & DAM & 87 & 2016.467 & 133.9 & 0.2 & 10.592 & 0.036 & 1 & \\
\hline $15597+6609$ & LDS & 1432 & 2016.467 & 48.3 & 0.2 & 5.609 & 0.019 & 1 & \\
\hline $16030+1359$ & STF & 2000 & 2016.544 & 228.7 & 0.2 & 2.628 & 0.009 & 1 & \\
\hline $16034+1025$ & $\mathrm{~J}$ & 446 & 2016.544 & 183.1 & 0.2 & 4.120 & 0.014 & 1 & \\
\hline $16062+1116$ & HEI & 540 & 2005.479 & 55.9 & 0.2 & 3.808 & 0.013 & 1 & $\mathrm{~A}$ \\
\hline $16070+6814$ & $\mathrm{UC}$ & 3117 & 2016.451 & 274.3 & 0.2 & 23.086 & 0.078 & 1 & \\
\hline $16071+5159$ & $\mathrm{UC}$ & 3119 & 2016.467 & 219.5 & 0.2 & 9.372 & 0.032 & 1 & \\
\hline $16091+4634$ & BEM & 9007 & 2016.467 & 133.3 & 0.2 & 13.574 & 0.046 & 1 & \\
\hline $16098+4624$ & BEM & 9008 & 2016.467 & 246.3 & 0.2 & 19.679 & 0.067 & 1 & \\
\hline $16116-2050$ & $\mathrm{UC}$ & 3130 & 2016.527 & 31.7 & 0.2 & 20.362 & 0.069 & 1 & \\
\hline $16120-1928$ & H 5 & $6 \mathrm{AB}, \mathrm{C}$ & 2016.437 & 337.6 & 0.2 & 41.645 & 0.142 & 1 & \\
\hline $16120-1928$ & MTL & $2 \mathrm{CD}$ & 2016.437 & 57.1 & 0.2 & 2.338 & 0.008 & 1 & \\
\hline $16121+4647$ & BEM & 9009 & 2016.494 & 294.3 & 0.2 & 5.643 & 0.019 & 1 & \\
\hline $16138+2844$ & STF & 2029 & 2016.544 & 189.2 & 0.2 & 6.156 & 0.021 & 1 & \\
\hline $16139+6420$ & MLR & 138 & 2016.451 & 319.1 & 0.2 & 2.554 & 0.009 & 1 & \\
\hline
\end{tabular}


Table 4-Continued

\begin{tabular}{|c|c|c|c|c|c|c|c|c|c|}
\hline $\begin{array}{l}\text { WDS Desig. } \\
\alpha, \delta(2000)\end{array}$ & $\begin{array}{l}\text { Dis } \\
\text { Desi }\end{array}$ & $\begin{array}{l}\text { coverer } \\
\text { gnation }\end{array}$ & JY & $\begin{array}{c}\theta \\
(0)\end{array}$ & $\begin{array}{l}\sigma \theta \\
\text { (०) }\end{array}$ & $\begin{array}{c}\rho \\
\left({ }^{\prime \prime}\right)\end{array}$ & $\begin{array}{l}\sigma \rho \\
\left({ }^{\prime \prime}\right)\end{array}$ & $\mathrm{n}$ & Note \\
\hline $16145+0531$ & STF & 2023 & 2016.544 & 225.6 & 0.2 & 1.949 & 0.007 & 1 & \\
\hline $16153+0416$ & STF & 2027 & 2016.544 & 83.3 & 0.2 & 1.891 & 0.006 & 1 & \\
\hline $16188+1724$ & STF & 2037 & 2016.544 & 257.1 & 0.2 & 1.139 & 0.004 & 1 & \\
\hline $16188+3154$ & $\mathrm{HU}$ & 1172 & 2016.544 & 329.0 & 0.2 & 1.768 & 0.006 & 1 & \\
\hline $16209+6422$ & STF & $2046 \mathrm{AB}$ & 2016.451 & 222.1 & 0.2 & 8.048 & 0.027 & 1 & \\
\hline $16222+6421$ & FOX & 17 & 2016.451 & 174.3 & 0.2 & 3.884 & 0.013 & 1 & \\
\hline $16222+6427$ & DAM & 349 & 2016.451 & 174.9 & 0.2 & 10.586 & 0.036 & 1 & \\
\hline $16233+3251$ & $\mathrm{COU}$ & 619 & 2016.544 & 201.6 & 0.2 & 0.552 & 0.002 & 1 & \\
\hline $16234+4251$ & ARG & 76 & 2016.494 & 252.6 & 0.2 & 58.057 & 0.197 & 1 & \\
\hline $16239+6020$ & ES & 1828 & 2016.451 & 248.1 & 0.2 & 3.995 & 0.014 & 1 & \\
\hline $16246+3632$ & $\mathrm{HO}$ & $405 \mathrm{AB}$ & 2016.495 & 345.6 & 0.2 & 4.402 & 0.015 & 1 & \\
\hline $16259+4820$ & $\mathrm{CBL}$ & 64 & 2016.494 & 152.7 & 0.2 & 31.143 & 0.106 & 1 & \\
\hline $16269+3801$ & ALI & 864 & 2016.494 & 59.4 & 0.2 & 8.414 & 0.029 & 1 & \\
\hline $16278+2506$ & $\mathrm{COU}$ & 413 & 2016.544 & 323.1 & 0.2 & 4.674 & 0.016 & 1 & \\
\hline $16279+2559$ & STF & 2049 & 2016.544 & 196.7 & 0.2 & 1.136 & 0.004 & 1 & \\
\hline $16286+4819$ & BEM & 9014 & 2016.451 & 109.9 & 0.2 & 14.410 & 0.049 & 1 & \\
\hline $16308+2744$ & $\mathrm{HO}$ & 64 & 2016.544 & 110.7 & 0.2 & 4.317 & 0.015 & 1 & \\
\hline $16326+0024$ & TOB & 260 & 2016.527 & 268.3 & 0.2 & 27.401 & 0.093 & 1 & \\
\hline $16348+0430$ & SKF & 13 & 2016.552 & 89.8 & 0.2 & 8.125 & 0.028 & 1 & \\
\hline $16352+3859$ & LDS & 985 & 2016.494 & 86.3 & 0.2 & 56.592 & 0.192 & 1 & \\
\hline $16381+0139$ & $\mathrm{AG}$ & 204 & 2016.552 & 16.3 & 0.2 & 4.314 & 0.015 & 1 & \\
\hline $16383+0753$ & $\mathrm{ROE}$ & 118 & 2016.552 & 38.7 & 0.2 & 6.187 & 0.021 & 1 & \\
\hline $16391+6042$ & STF & 2092 & 2016.555 & 7.0 & 0.2 & 8.363 & 0.028 & 1 & \\
\hline $16397+6738$ & $\mathrm{UC}$ & 3201 & 2016.451 & 151.0 & 0.2 & 15.905 & 0.054 & 1 & \\
\hline $16405-0404$ & $\mathrm{~J}$ & 2110 & 2016.527 & 173.8 & 0.2 & 5.156 & 0.018 & 1 & \\
\hline 16407-0009 & STF & $2076 \mathrm{AB}$ & 2016.527 & 324.6 & 0.2 & 9.517 & 0.032 & 1 & \\
\hline $16407+0547$ & $\mathrm{~J}$ & $447 \mathrm{AB}$ & 2016.552 & 241.1 & 0.2 & 5.506 & 0.019 & 1 & \\
\hline $16415+2147$ & $\mathrm{~J}$ & 738 & 2005.354 & 247.4 & 0.2 & 2.085 & 0.007 & 1 & $\mathrm{~A}$ \\
\hline $16420+4031$ & BRT & 339 & 2016.494 & 281.4 & 0.2 & 4.795 & 0.016 & 1 & \\
\hline $16443-0407$ & HJ & 4886 & 2016.527 & 98.7 & 0.2 & 7.958 & 0.027 & 1 & \\
\hline $16444+4346$ & TOB & 135 & 2016.467 & 255.9 & 0.2 & 20.816 & 0.071 & 1 & \\
\hline $16445-0205$ & BAL & 569 & 2016.527 & 119.6 & 0.2 & 4.421 & 0.015 & 1 & \\
\hline
\end{tabular}


Table 4-Continued

\begin{tabular}{|c|c|c|c|c|c|c|c|c|c|}
\hline $\begin{array}{l}\text { WDS Desig. } \\
\alpha, \delta(2000)\end{array}$ & $\begin{array}{l}\text { Di } \\
\text { Des }\end{array}$ & $\begin{array}{l}\text { coverer } \\
\text { ignation }\end{array}$ & JY & $\begin{array}{c}\theta \\
(0)\end{array}$ & $\begin{array}{l}\sigma \theta \\
(0)\end{array}$ & $\begin{array}{c}\rho \\
\left({ }^{\prime \prime}\right)\end{array}$ & $\begin{array}{l}\sigma \rho \\
\left({ }^{\prime \prime}\right)\end{array}$ & $\mathrm{n}$ & Note \\
\hline $16456+0304$ & BAL & 2423 & 2016.552 & 293.9 & 0.2 & 4.630 & 0.016 & 1 & \\
\hline $16462-1721$ & SKI & 10 & 2016.527 & 87.1 & 0.2 & 3.348 & 0.011 & 1 & \\
\hline $16481+0500$ & BRT & 2167 & 2016.552 & 228.1 & 0.2 & 3.129 & 0.011 & 1 & \\
\hline $16483+0244$ & $\mathrm{BU}$ & 43 & 2016.552 & 56.9 & 0.2 & 1.414 & 0.005 & 1 & \\
\hline $16486+0545$ & $\mathrm{UC}$ & 3237 & 2016.552 & 86.5 & 0.2 & 23.579 & 0.080 & 1 & \\
\hline $16490+0315$ & $\mathrm{KU}$ & 114 & 2016.552 & 113.4 & 0.2 & 60.608 & 0.206 & 1 & \\
\hline $16491+6203$ & STI & $807 \mathrm{AB}$ & 2016.555 & 249.1 & 0.2 & 9.052 & 0.031 & 1 & \\
\hline $16497+6844$ & WFC & 180 & 2016.467 & 123.6 & 0.2 & 4.703 & 0.016 & 1 & \\
\hline $16536+0254$ & BAL & 2428 & 2016.434 & 279.1 & 0.2 & 11.023 & 0.037 & 1 & \\
\hline $16541+0826$ & HEI & 857 & 2016.552 & 138.4 & 0.2 & 0.489 & 0.002 & 1 & \\
\hline $16548-1133$ & HU & 159 & 2016.527 & 153.2 & 0.2 & 4.195 & 0.014 & 1 & \\
\hline $16552+6332$ & STF & 2116 & 2016.555 & 6.0 & 0.2 & 19.243 & 0.065 & 1 & \\
\hline $16556-0510$ & STF & 3106 & 2016.527 & 81.1 & 0.2 & 1.878 & 0.006 & 1 & \\
\hline $16556+6102$ & STI & 810 & 2005.245 & 121.3 & 0.2 & 12.953 & 0.044 & 1 & A \\
\hline $16561+0241$ & BAL & 1929 & 2016.552 & 323.4 & 0.2 & 12.932 & 0.044 & 1 & \\
\hline $16568+2734$ & BRT & 172 & 2016.434 & 217.3 & 0.2 & 4.820 & 0.016 & 1 & \\
\hline $16571-1932$ & SHJ & 240 & 2016.527 & 235.2 & 0.2 & 4.624 & 0.016 & 1 & \\
\hline $16575+6349$ & $\mathrm{UC}$ & 3263 & 2016.467 & 181.8 & 0.2 & 13.705 & 0.047 & 1 & \\
\hline $16580+0139$ & BAL & 1480 & 2016.527 & 285.7 & 0.2 & 16.472 & 0.056 & 1 & \\
\hline $16588+0358$ & STF & $3107 \mathrm{AB}$ & 2016.552 & 72.6 & 0.2 & 1.362 & 0.005 & 1 & \\
\hline $16593-1312$ & HLD & 27 & 2016.527 & 137.0 & 0.2 & 4.888 & 0.017 & 1 & \\
\hline $16593+6513$ & STF & 2124 & 2016.555 & 88.9 & 0.2 & 14.884 & 0.051 & 1 & \\
\hline $17010+6807$ & WSI & $35 \mathrm{AD}$ & 2016.495 & 84.6 & 0.2 & 7.002 & 0.024 & 1 & \\
\hline $17017+0701$ & DAM & 699 & 2016.552 & 278.6 & 0.2 & 21.911 & 0.074 & 1 & \\
\hline $17020+0827$ & STF & 2114 & 2016.552 & 198.9 & 0.2 & 1.365 & 0.005 & 1 & \\
\hline $17021+0713$ & STF & 2113 & 2016.552 & 118.6 & 0.2 & 5.120 & 0.017 & 1 & \\
\hline $17022+3807$ & ALI & 865 & 2016.467 & 134.4 & 0.2 & 11.456 & 0.039 & 1 & \\
\hline $17055+6316$ & STI & 815 & 2016.467 & 81.9 & 0.2 & 7.175 & 0.024 & 1 & \\
\hline $17056+0530$ & SKF & 14 & 2016.659 & 261.7 & 0.2 & 39.625 & 0.135 & 1 & \\
\hline $17065-1356$ & STF & 2119 & 2016.656 & 184.6 & 0.2 & 2.432 & 0.008 & 1 & \\
\hline $17071+6915$ & WFC & 187 & 2016.495 & 225.9 & 0.2 & 3.641 & 0.012 & 1 & \\
\hline $17076+0332$ & BAL & 2434 & 2016.659 & 316.9 & 0.2 & 15.539 & 0.053 & 1 & \\
\hline
\end{tabular}


Table 4-Continued

\begin{tabular}{|c|c|c|c|c|c|c|c|c|c|}
\hline $\begin{array}{l}\text { WDS Desig. } \\
\alpha, \delta(2000)\end{array}$ & $\begin{array}{l}\text { Dis } \\
\text { Desi }\end{array}$ & $\begin{array}{l}\text { coverer } \\
\text { ignation }\end{array}$ & JY & $\begin{array}{c}\theta \\
(0)\end{array}$ & $\begin{array}{l}\sigma \theta \\
(0)\end{array}$ & $\begin{array}{c}\rho \\
\left({ }^{\prime \prime}\right)\end{array}$ & $\begin{array}{l}\sigma \rho \\
\left({ }^{\prime \prime}\right)\end{array}$ & $\mathrm{n}$ & Note \\
\hline $17091+8456$ & WFC & 188 & 2016.495 & 174.5 & 0.2 & 11.296 & 0.038 & 1 & \\
\hline $17100-1604$ & FOX & 197 & 2016.656 & 112.3 & 0.2 & 14.542 & 0.049 & 1 & \\
\hline $17109+4357$ & ES & 1412 & 2016.495 & 134.1 & 0.2 & 3.720 & 0.013 & 1 & \\
\hline $17113+6930$ & STF & 2151 & 2016.495 & 350.3 & 0.2 & 2.467 & 0.008 & 1 & \\
\hline $17126+7108$ & PRZ & 13 & 2016.555 & 138.7 & 0.2 & 25.122 & 0.085 & 1 & \\
\hline $17128-0403$ & STF & 2132 & 2016.656 & 113.6 & 0.2 & 1.993 & 0.007 & 1 & \\
\hline $17131+5408$ & STF & $2146 \mathrm{AC}$ & 2016.495 & 234.8 & 0.2 & 89.258 & 0.303 & 1 & \\
\hline $17134-1839$ & ARA & 438 & 2016.656 & 262.3 & 0.2 & 13.336 & 0.045 & 1 & \\
\hline $17139+1557$ & STF & 2137 & 2016.661 & 144.3 & 0.2 & 4.119 & 0.014 & 1 & \\
\hline $17142+6904$ & $\mathrm{UC}$ & 225 & 2016.495 & 341.3 & 0.2 & 51.401 & 0.175 & 1 & \\
\hline $17153+7239$ & HJ & 3346 & 2016.495 & 29.6 & 0.2 & 13.351 & 0.045 & 1 & \\
\hline $17157-1811$ & HJ & $4932 \mathrm{AC}$ & 2016.656 & 226.5 & 0.2 & 10.468 & 0.036 & 1 & \\
\hline $17157-1811$ & WHC & $14 \mathrm{AB}$ & 2016.656 & 291.0 & 0.2 & 1.203 & 0.004 & 1 & \\
\hline $17160+2437$ & $\mathrm{POU}$ & 3273 & 2016.437 & 253.1 & 0.2 & 8.553 & 0.029 & 1 & \\
\hline $17165-1920$ & $\mathrm{BU}$ & 958 & 2016.656 & 211.0 & 0.2 & 1.936 & 0.007 & 1 & \\
\hline $17170+3000$ & GRV & 949 & 2016.437 & 277.9 & 0.2 & 10.563 & 0.036 & 1 & \\
\hline $17174+0358$ & DOO & 65 & 2016.437 & 28.7 & 0.2 & 3.048 & 0.010 & 1 & \\
\hline $17178+0733$ & $\mathrm{~J}$ & 450 & 2016.659 & 60.6 & 0.2 & 4.665 & 0.016 & 1 & \\
\hline $17196+3252$ & SEI & 542 & 2016.437 & 154.6 & 0.2 & 21.052 & 0.072 & 1 & \\
\hline $17200-0626$ & STF & 2149 & 2016.656 & 23.7 & 0.2 & 7.359 & 0.025 & 1 & \\
\hline $17205+6315$ & ES & 1909 & 2016.555 & 255.3 & 0.2 & 6.239 & 0.021 & 1 & \\
\hline $17208-0902$ & A & 28 & 2016.656 & 36.5 & 0.2 & 2.043 & 0.007 & 1 & \\
\hline $17220+5638$ & ES & $2657 \mathrm{AB}$ & 2016.495 & 97.5 & 0.2 & 28.396 & 0.097 & 1 & \\
\hline $17220+5638$ & ES & $2657 \mathrm{AC}$ & 2016.495 & 90.8 & 0.2 & 39.179 & 0.133 & 1 & \\
\hline $17220+5638$ & $\mathrm{ES}$ & $2657 \mathrm{BC}$ & 2016.495 & 74.1 & 0.2 & 11.736 & 0.040 & 1 & \\
\hline $17237+4717$ & STF & $2164 \mathrm{AB}$ & 2016.495 & 13.9 & 0.2 & 9.367 & 0.032 & 1 & \\
\hline $17240-0050$ & STF & 2156 & 2016.658 & 35.8 & 0.2 & 3.844 & 0.013 & 1 & \\
\hline $17240-1142$ & $\mathrm{BU}$ & $242 \mathrm{AB}$ & 2016.656 & 78.8 & 0.2 & 1.156 & 0.004 & 1 & \\
\hline $17240-1142$ & $\mathrm{BU}$ & $242 \mathrm{AD}$ & 2016.656 & 61.6 & 0.2 & 48.511 & 0.165 & 1 & \\
\hline $17267+4727$ & BEM & $9011 \mathrm{AB}$ & 2016.495 & 210.2 & 0.2 & 10.441 & 0.035 & 1 & \\
\hline $17278+6757$ & TDT & 366 & 2016.495 & 6.2 & 0.2 & 4.329 & 0.015 & 1 & \\
\hline $17291+1727$ & DAL & $14 \mathrm{AC}$ & 2016.437 & 185.0 & 0.2 & 18.404 & 0.063 & 1 & \\
\hline
\end{tabular}


Table 4-Continued

\begin{tabular}{|c|c|c|c|c|c|c|c|c|c|}
\hline $\begin{array}{l}\text { WDS Desig. } \\
\alpha, \delta(2000)\end{array}$ & $\begin{array}{l}\text { Dis } \\
\text { Desi }\end{array}$ & $\begin{array}{l}\text { coverer } \\
\text { gnation }\end{array}$ & JY & $\begin{array}{l}\theta \\
(0)\end{array}$ & $\begin{array}{l}\sigma \theta \\
(0)\end{array}$ & $\begin{array}{c}\rho \\
\left({ }^{\prime \prime}\right)\end{array}$ & $\begin{array}{l}\sigma \rho \\
\left({ }^{\prime \prime}\right)\end{array}$ & $\mathrm{n}$ & Note \\
\hline 17293-0959 & STF & 2171 & 2016.656 & 59.8 & 0.2 & 1.475 & 0.005 & 1 & \\
\hline $17309+0226$ & BAL & 1938 & 2016.658 & 117.8 & 0.2 & 11.929 & 0.041 & 1 & \\
\hline $17317+1111$ & $\mathrm{AG}$ & 354 & 2016.642 & 51.7 & 0.2 & 2.483 & 0.008 & 1 & \\
\hline $17323-0828$ & HJ & 4960 & 2016.656 & 93.0 & 0.2 & 6.144 & 0.021 & 1 & \\
\hline $17334+7414$ & LDS & 1871 & 2016.555 & 232.3 & 0.2 & 33.272 & 0.113 & 1 & \\
\hline $17342+0235$ & BAL & 1940 & 2016.659 & 138.1 & 0.2 & 15.812 & 0.054 & 1 & \\
\hline $17346+0935$ & STFA & $34 \mathrm{AB}$ & 2016.642 & 190.2 & 0.2 & 41.416 & 0.141 & 1 & \\
\hline $17358+0100$ & STF & 2186 & 2016.658 & 77.7 & 0.2 & 3.045 & 0.010 & 1 & \\
\hline $17387+0349$ & BAL & 2445 & 2016.544 & 142.2 & 0.2 & 5.094 & 0.017 & 1 & \\
\hline $17406+2017$ & $\mathrm{AG}$ & 211 & 2016.544 & 131.1 & 0.2 & 2.718 & 0.009 & 1 & \\
\hline $17411+2431$ & STF & $2194 \mathrm{AB}$ & 2016.544 & 9.3 & 0.2 & 16.489 & 0.056 & 1 & \\
\hline $17420+0814$ & STF & 2193 & 2016.544 & 67.6 & 0.2 & 5.899 & 0.020 & 1 & \\
\hline $17420+2127$ & STF & 2197 & 2016.544 & 358.5 & 0.2 & 8.077 & 0.027 & 1 & \\
\hline $17439+0551$ & STF & $2200 \mathrm{AB}$ & 2016.544 & 165.3 & 0.2 & 1.599 & 0.005 & 1 & \\
\hline $17439+1455$ & $\mathrm{~J}$ & 2115 & 2016.544 & 225.6 & 0.2 & 6.642 & 0.023 & 1 & \\
\hline $17444+0258$ & STF & 2201 & 2016.544 & 304.5 & 0.2 & 7.821 & 0.027 & 1 & \\
\hline $17446+0235$ & STF & $2202 \mathrm{AB}$ & 2016.544 & 95.3 & 0.2 & 20.949 & 0.071 & 1 & \\
\hline $17464+0542$ & STF & 2212 & 2016.544 & 343.1 & 0.2 & 3.313 & 0.011 & 1 & \\
\hline $17487+0110$ & STF & 2221 & 2016.642 & 76.3 & 0.2 & 19.516 & 0.066 & 1 & \\
\hline $17504+0433$ & BAL & $2902 \mathrm{AB}$ & 2016.544 & 50.6 & 0.2 & 3.278 & 0.011 & 1 & \\
\hline $17504+0433$ & BAL & $2902 \mathrm{AC}$ & 2016.544 & 50.2 & 0.2 & 21.552 & 0.073 & 1 & \\
\hline $17504+0433$ & BAL & $2902 \mathrm{BC}$ & 2016.544 & 50.2 & 0.2 & 18.465 & 0.063 & 1 & \\
\hline $17523+6414$ & $\mathrm{KR}$ & 47 & 2016.555 & 204.6 & 0.2 & 7.602 & 0.026 & 1 & \\
\hline $17527+1940$ & BRT & 2439 & 2016.544 & 182.9 & 0.2 & 4.781 & 0.016 & 1 & \\
\hline $17549+2347$ & $\mathrm{POU}$ & 3323 & 2016.544 & 191.5 & 0.2 & 8.970 & 0.030 & 1 & \\
\hline $17555+2250$ & GRV & 970 & 2016.642 & 153.7 & 0.2 & 44.379 & 0.151 & 1 & \\
\hline $17573+1531$ & $\mathrm{ROE}$ & 120 & 2016.552 & 237.9 & 0.2 & 6.571 & 0.022 & 1 & \\
\hline $17590+0202$ & STF & $2252 \mathrm{AB}$ & 2016.552 & 25.8 & 0.2 & 4.027 & 0.014 & 1 & \\
\hline $17590+1226$ & STF & $2254 \mathrm{AB}$ & 2016.552 & 267.9 & 0.2 & 3.555 & 0.012 & 1 & \\
\hline $18000+2449$ & $\mathrm{COU}$ & 115 & 2016.645 & 112.8 & 0.2 & 0.333 & 0.001 & 1 & \\
\hline $18012+0324$ & BAL & 2465 & 2016.552 & 289.2 & 0.2 & 19.262 & 0.065 & 1 & \\
\hline $18015+2136$ & STF & 2264 & 2016.552 & 258.9 & 0.2 & 6.427 & 0.022 & 1 & \\
\hline
\end{tabular}


Table 4-Continued

\begin{tabular}{|c|c|c|c|c|c|c|c|c|c|}
\hline \multirow{2}{*}{$\begin{array}{c}\text { WDS Desig. } \\
\alpha, \delta(2000) \\
18032+2522\end{array}$} & \multicolumn{2}{|c|}{$\begin{array}{l}\text { Discoverer } \\
\text { Designation }\end{array}$} & \multirow{2}{*}{$\begin{array}{c}\text { JY } \\
2016.645\end{array}$} & \multirow{2}{*}{$\begin{array}{c}\theta \\
(0) \\
185.5\end{array}$} & \multirow{2}{*}{$\begin{array}{l}\sigma \theta \\
(\circ) \\
0.2\end{array}$} & \multirow{2}{*}{$\begin{array}{c}\rho \\
\left({ }^{\prime \prime}\right) \\
16.734\end{array}$} & \multirow{2}{*}{$\begin{array}{l}\sigma \rho \\
\left({ }^{\prime \prime}\right) \\
0.057\end{array}$} & \multirow{2}{*}{$\begin{array}{l}\mathrm{n} \\
\mathrm{T} \\
1\end{array}$} & \multirow{2}{*}{$\begin{array}{l}\text { Note } \\
\end{array}$} \\
\hline & $\mathrm{BU}$ & $825 \mathrm{AB}$ & & & & & & & \\
\hline $18032+2522$ & $\mathrm{BU}$ & $825 \mathrm{BC}$ & 2016.645 & 231.1 & 0.2 & 9.757 & 0.033 & 1 & \\
\hline $18044+0329$ & STF & $2266 \mathrm{AB}$ & 2016.552 & 187.5 & 0.2 & 8.673 & 0.029 & 1 & \\
\hline $18048+2353$ & STF & 2274 & 2016.642 & 293.5 & 0.2 & 18.586 & 0.063 & 1 & \\
\hline $18056+0051$ & $\mathrm{~J}$ & 518 & 2016.552 & 142.4 & 0.2 & 5.076 & 0.017 & 1 & \\
\hline $18061-0022$ & A & 1167 & 2016.552 & 149.7 & 0.2 & 5.111 & 0.017 & 1 & \\
\hline $18065+0331$ & SLE & 84 & 2016.552 & 179.6 & 0.2 & 20.324 & 0.069 & 1 & \\
\hline $18065+0920$ & ARN & $9001 \mathrm{AB}$ & 2016.552 & 114.4 & 0.2 & 38.235 & 0.130 & 1 & \\
\hline $18078+2606$ & STF & $2280 \mathrm{AB}$ & 2016.645 & 183.0 & 0.2 & 14.376 & 0.049 & 1 & \\
\hline $18095+6414$ & $\mathrm{HU}$ & 926 & 2016.653 & 264.9 & 0.2 & 3.890 & 0.013 & 1 & \\
\hline $18103+0209$ & DAL & $18 \mathrm{AB}$ & 2016.552 & 274.4 & 0.2 & 12.535 & 0.043 & 1 & \\
\hline $18103+0209$ & DAL & $18 \mathrm{AC}$ & 2016.552 & 314.3 & 0.2 & 45.047 & 0.153 & 1 & \\
\hline $18106+0349$ & FOX & 220 & 2016.552 & 77.0 & 0.2 & 5.995 & 0.020 & 1 & \\
\hline $18107+0417$ & BAL & 2917 & 2016.552 & 258.6 & 0.2 & 19.698 & 0.067 & 1 & \\
\hline $18122+1935$ & BRT & 2443 & 2016.645 & 117.6 & 0.2 & 6.293 & 0.021 & 1 & \\
\hline $18130+0243$ & BAL & $1959 \mathrm{AB}$ & 2016.642 & 31.5 & 0.2 & 23.067 & 0.078 & 1 & \\
\hline $18130+0243$ & BAL & $1959 \mathrm{AC}$ & 2016.642 & 66.5 & 0.2 & 15.437 & 0.052 & 1 & \\
\hline $18130+0243$ & BAL & $1959 \mathrm{BC}$ & 2016.642 & 171.5 & 0.2 & 13.750 & 0.047 & 1 & \\
\hline $18142+2127$ & BIG & 2 & 2016.645 & 141.1 & 0.2 & 34.484 & 0.117 & 1 & \\
\hline $18144+0415$ & SLE & 143 & 2016.645 & 41.8 & 0.2 & 15.208 & 0.052 & 1 & \\
\hline $18144+2127$ & HJ & 2828 & 2016.645 & 109.8 & 0.2 & 16.438 & 0.056 & 1 & \\
\hline $18146-1113$ & HLD & 142 & 2016.642 & 254.1 & 0.2 & 1.192 & 0.004 & 1 & \\
\hline $18148-0441$ & HJ & 856 & 2016.642 & 235.4 & 0.2 & 22.200 & 0.075 & 1 & \\
\hline $18151+0448$ & BAL & 2923 & 2016.645 & 17.6 & 0.2 & 16.146 & 0.055 & 1 & \\
\hline $18152+0229$ & BAL & 1961 & 2016.642 & 359.1 & 0.2 & 17.436 & 0.059 & 1 & \\
\hline $18153+0305$ & SLE & $146 \mathrm{AB}$ & 2016.645 & 151.5 & 0.2 & 18.410 & 0.063 & 1 & \\
\hline $18153+0305$ & SLE & $146 \mathrm{AC}$ & 2016.645 & 157.8 & 0.2 & 49.779 & 0.169 & 1 & \\
\hline $18154+1946$ & STT & 346 & 2016.645 & 329.0 & 0.2 & 5.231 & 0.018 & 1 & \\
\hline $18157+0400$ & BAL & $2484 \mathrm{AB}$ & 2016.645 & 357.2 & 0.2 & 8.000 & 0.027 & 1 & \\
\hline $18157+0400$ & SLE & $147 \mathrm{AC}$ & 2016.645 & 326.0 & 0.2 & 30.541 & 0.104 & 1 & \\
\hline $18162+0434$ & STT & A167 & 2016.645 & 74.0 & 0.2 & 53.031 & 0.180 & 1 & \\
\hline $18177-1940$ & $\mathrm{BU}$ & $246 \mathrm{AB}$ & 2016.653 & 114.2 & 0.2 & 0.487 & 0.002 & 1 & \\
\hline
\end{tabular}


Table 4-Continued

\begin{tabular}{|c|c|c|c|c|c|c|c|c|c|}
\hline $\begin{array}{l}\text { WDS Desig. } \\
\alpha, \delta(2000)\end{array}$ & \multicolumn{2}{|c|}{$\begin{array}{l}\text { Discoverer } \\
\text { Designation }\end{array}$} & JY & $\begin{array}{c}\theta \\
(0)\end{array}$ & $\begin{array}{l}\sigma \theta \\
(0)\end{array}$ & $\begin{array}{c}\rho \\
\left({ }^{\prime \prime}\right)\end{array}$ & $\begin{array}{l}\sigma \rho \\
(")\end{array}$ & $\mathrm{n}$ & Note \\
\hline $18179-1848$ & SHJ & $263 \mathrm{AB}$ & 2016.653 & 11.3 & 0.2 & 53.879 & 0.183 & 1 & \\
\hline $18180-1842$ & HWE & 42 & 2016.653 & 191.6 & 0.2 & 21.199 & 0.072 & 1 & \\
\hline $18187-1837$ & SHJ & $264 \mathrm{AB}, \mathrm{C}$ & 2016.653 & 51.2 & 0.2 & 17.342 & 0.059 & 1 & \\
\hline $18192-1757$ & ARA & 276 & 2016.653 & 40.8 & 0.2 & 12.621 & 0.043 & 1 & \\
\hline $18195-1754$ & ARA & 277 & 2016.653 & 117.9 & 0.2 & 15.075 & 0.051 & 1 & \\
\hline $18210-1939$ & $\mathrm{BU}$ & $48 \mathrm{CD}$ & 2016.653 & 58.0 & 0.2 & 16.375 & 0.056 & 1 & \\
\hline $18232-1823$ & TOB & 288 & 2016.653 & 185.0 & 0.2 & 12.616 & 0.043 & 1 & \\
\hline $18237-1936$ & ARA & 746 & 2016.653 & 281.1 & 0.2 & 12.911 & 0.044 & 1 & \\
\hline $18260+0803$ & SLE & $175 \mathrm{AB}$ & 2016.664 & 105.2 & 0.2 & 15.511 & 0.053 & 1 & \\
\hline $18260+0803$ & SLE & $175 \mathrm{AC}$ & 2016.664 & 48.0 & 0.2 & 19.699 & 0.067 & 1 & \\
\hline $18262+0653$ & $\mathrm{~J}$ & 1830 & 2016.664 & 270.8 & 0.2 & 7.382 & 0.025 & 1 & \\
\hline $18265+0741$ & A & 580 & 2016.664 & 323.0 & 0.2 & 4.568 & 0.016 & 1 & \\
\hline $18266+0627$ & GCB & 31 & 2016.664 & 76.2 & 0.2 & 4.507 & 0.015 & 1 & \\
\hline $18276+0633$ & $\mathrm{BU}$ & $464 \mathrm{AB}$ & 2016.664 & 105.2 & 0.2 & 1.055 & 0.004 & 1 & \\
\hline $18281+0709$ & $\mathrm{~J}$ & $95 \mathrm{AB}$ & 2016.664 & 123.4 & 0.2 & 2.151 & 0.007 & 1 & \\
\hline $18281+0709$ & $\mathrm{~J}$ & $95 \mathrm{AC}$ & 2016.664 & 32.9 & 0.2 & 31.967 & 0.109 & 1 & \\
\hline $18284+0407$ & $\mathrm{~J}$ & 521 & 2016.664 & 304.5 & 0.2 & 5.262 & 0.018 & 1 & \\
\hline $18286+0451$ & STT & A168 AB & 2016.664 & 161.6 & 0.2 & 47.190 & 0.160 & 1 & \\
\hline $18314+0628$ & STF & 2329 & 2016.664 & 42.9 & 0.2 & 4.286 & 0.015 & 1 & \\
\hline $18316+0732$ & $\mathrm{~J}$ & 1149 & 2016.664 & 201.3 & 0.2 & 2.674 & 0.009 & 1 & \\
\hline $18318+0729$ & SLE & 350 & 2016.664 & 144.7 & 0.2 & 16.821 & 0.057 & 1 & \\
\hline $18328+1349$ & STF & 2336 & 2016.664 & 8.6 & 0.2 & 6.774 & 0.023 & 1 & \\
\hline $18329+0539$ & SLE & 495 & 2016.664 & 99.5 & 0.2 & 8.525 & 0.029 & 1 & \\
\hline $18329+0917$ & WFC & 209 & 2016.664 & 337.4 & 0.2 & 7.181 & 0.024 & 1 & \\
\hline $18335+0726$ & SCJ & $18 \mathrm{AB}$ & 2016.664 & 197.0 & 0.2 & 45.979 & 0.156 & 1 & \\
\hline $18370+3452$ & STF & $2352 \mathrm{AB}$ & 2016.689 & 287.3 & 0.2 & 15.915 & 0.054 & 1 & \\
\hline $18421+3445$ & STF & $2372 \mathrm{AB}$ & 2016.689 & 82.4 & 0.2 & 25.161 & 0.086 & 1 & \\
\hline $18427+1152$ & $\mathrm{AG}$ & $364 \mathrm{BC}$ & 2004.644 & 143.8 & 0.2 & 8.405 & 0.029 & 1 & $\mathrm{~A}$ \\
\hline $18448+3736$ & STFA & $38 \mathrm{AD}$ & 2016.699 & 150.0 & 0.2 & 43.900 & 0.149 & 1 & \\
\hline $18453+1140$ & $\mathrm{~J}$ & 2145 & 2016.648 & 117.1 & 0.2 & 5.376 & 0.018 & 1 & \\
\hline $18455+0530$ & STF & $2375 \mathrm{AB}$ & 2016.648 & 120.5 & 0.2 & 2.612 & 0.009 & 1 & \\
\hline $18457-0107$ & BAL & 582 & 2016.648 & 321.5 & 0.2 & 12.364 & 0.042 & 1 & \\
\hline
\end{tabular}


Table 4-Continued

\begin{tabular}{|c|c|c|c|c|c|c|c|c|c|}
\hline $\begin{array}{l}\text { WDS Desig. } \\
\alpha, \delta(2000)\end{array}$ & & $\begin{array}{l}\text { iscoverer } \\
\text { signation }\end{array}$ & JY & $\begin{array}{c}\theta \\
(0)\end{array}$ & $\begin{array}{l}\sigma \theta \\
(0)\end{array}$ & $\begin{array}{c}\rho \\
\left({ }^{\prime \prime}\right)\end{array}$ & $\begin{array}{l}\sigma \rho \\
\left({ }^{\prime \prime}\right)\end{array}$ & $\mathrm{n}$ & Note \\
\hline $18458+3431$ & STF & 2390 & 2016.683 & 155.2 & 0.2 & 4.253 & 0.014 & 1 & \\
\hline $18459-1030$ & STF & 2373 & 2016.648 & 337.4 & 0.2 & 4.172 & 0.014 & 1 & \\
\hline $18465-0058$ & STF & $2379 \mathrm{AB}$ & 2016.648 & 120.9 & 0.2 & 12.690 & 0.043 & 1 & \\
\hline $18473-0346$ & $\mathrm{~J}$ & 2232 & 2016.648 & 87.4 & 0.2 & 5.436 & 0.018 & 1 & \\
\hline $18477+3600$ & ALI & 142 & 2016.683 & 126.7 & 0.2 & 12.741 & 0.043 & 1 & \\
\hline $18487+3401$ & $\mathrm{HU}$ & 936 & 2016.683 & 98.8 & 0.2 & 1.873 & 0.006 & 1 & \\
\hline $18488-0120$ & BAL & 585 & 2016.648 & 148.8 & 0.2 & 4.648 & 0.016 & 1 & \\
\hline $18489+1615$ & STF & $2400 \mathrm{BC}$ & 2016.648 & 201.3 & 0.2 & 0.995 & 0.003 & 1 & \\
\hline $18495+3316$ & STF & 2407 & 2016.683 & 207.6 & 0.2 & 29.978 & 0.102 & 1 & \\
\hline $18508+1059$ & $\mathrm{STF}$ & 2404 & 2016.648 & 181.4 & 0.2 & 3.603 & 0.012 & 1 & \\
\hline $18518-0505$ & WFC & 213 & 2016.648 & 308.3 & 0.2 & 8.917 & 0.030 & 1 & \\
\hline $18520+1047$ & STF & 2408 & 2016.648 & 91.1 & 0.2 & 2.333 & 0.008 & 1 & \\
\hline $18523+1432$ & STF & $2411 \mathrm{AB}$ & 2016.648 & 95.4 & 0.2 & 13.534 & 0.046 & 1 & \\
\hline $18523+1432$ & WAL & $97 \mathrm{AC}$ & 2016.648 & 87.5 & 0.2 & 60.446 & 0.206 & 1 & \\
\hline $18523+1432$ & WAL & $97 \mathrm{AD}$ & 2016.648 & 118.3 & 0.2 & 84.148 & 0.286 & 1 & \\
\hline $18535+0912$ & ROE & $112 \mathrm{AB}$ & 2016.648 & 239.7 & 0.2 & 7.293 & 0.025 & 1 & \\
\hline $18535+0912$ & $\mathrm{ROE}$ & $112 \mathrm{AC}$ & 2016.648 & 226.2 & 0.2 & 43.327 & 0.147 & 1 & \\
\hline $18535+0912$ & $\mathrm{ROE}$ & $112 \mathrm{BC}$ & 2016.648 & 223.5 & 0.2 & 36.291 & 0.123 & 1 & \\
\hline $18535+7547$ & STF & 2452 & 2016.699 & 218.1 & 0.2 & 5.671 & 0.019 & 1 & \\
\hline $18544-0352$ & $\mathrm{~J}$ & 1374 & 2016.648 & 177.3 & 0.2 & 5.388 & 0.018 & 1 & \\
\hline $18549+3358$ & SHJ & $282 \mathrm{AC}$ & 2016.683 & 349.4 & 0.2 & 45.513 & 0.155 & 1 & \\
\hline $18551+1644$ & WFC & 216 & 2016.648 & 88.7 & 0.2 & 12.031 & 0.041 & 1 & \\
\hline $18554+1331$ & $\mathrm{BU}$ & $647 \mathrm{AB}$ & 2016.648 & 5.9 & 0.2 & 1.312 & 0.004 & 1 & \\
\hline $18554+1331$ & $\mathrm{BU}$ & $647 \mathrm{AC}$ & 2016.648 & 218.4 & 0.2 & 18.546 & 0.063 & 1 & \\
\hline $18558+1935$ & $\mathrm{KU}$ & 122 & 2016.648 & 151.2 & 0.2 & 22.249 & 0.076 & 1 & \\
\hline $18562+0412$ & STF & $2417 \mathrm{AB}$ & 2016.648 & 103.9 & 0.2 & 22.641 & 0.077 & 1 & \\
\hline $18581+0559$ & $\mathrm{OL}$ & 215 & 2016.648 & 104.4 & 0.2 & 5.723 & 0.019 & 1 & \\
\hline $18584+3625$ & STF & 2429 & 2016.683 & 287.3 & 0.2 & 5.509 & 0.019 & 1 & \\
\hline $18586+1242$ & $\mathrm{AG}$ & 369 & 2016.544 & 229.7 & 0.2 & 4.705 & 0.016 & 1 & \\
\hline $18590-0139$ & BAL & 588 & 2016.642 & 317.2 & 0.2 & 9.869 & 0.034 & 1 & \\
\hline $18590+0330$ & ALL & $3 \mathrm{~A}, \mathrm{BC}$ & 2016.642 & 48.1 & 0.2 & 34.679 & 0.118 & 1 & \\
\hline $19008+2318$ & HJ & 2850 & 2016.544 & 275.8 & 0.2 & 2.698 & 0.009 & 1 & \\
\hline
\end{tabular}


Table 4-Continued

\begin{tabular}{|c|c|c|c|c|c|c|c|c|c|}
\hline $\begin{array}{l}\text { WDS Desig. } \\
\alpha, \delta(2000)\end{array}$ & \multicolumn{2}{|c|}{$\begin{array}{l}\text { Discoverer } \\
\text { Designation }\end{array}$} & JY & $\begin{array}{c}\theta \\
(0)\end{array}$ & $\begin{array}{l}\sigma \theta \\
(0)\end{array}$ & $\begin{array}{c}\rho \\
\left({ }^{\prime \prime}\right)\end{array}$ & $\begin{array}{l}\sigma \rho \\
\left({ }^{\prime \prime}\right)\end{array}$ & $\mathrm{n}$ & Note \\
\hline $19031+0725$ & HJ & 2852 & 2016.642 & 128.3 & 0.2 & 22.035 & 0.075 & 1 & \\
\hline $19041+1106$ & $\mathrm{AG}$ & 371 & 2016.642 & 159.6 & 0.2 & 5.519 & 0.019 & 1 & \\
\hline $19041+1447$ & STF & 2443 & 2016.544 & 313.3 & 0.2 & 6.918 & 0.024 & 1 & \\
\hline $19043+1130$ & $\mathrm{~J}$ & $2163 \mathrm{AB}$ & 2016.544 & 25.3 & 0.2 & 52.141 & 0.177 & 1 & \\
\hline $19050-0402$ & SHJ & 286 & 2016.642 & 210.0 & 0.2 & 39.663 & 0.135 & 1 & \\
\hline $19052+1050$ & $\mathrm{BU}$ & 466 & 2016.642 & 164.5 & 0.2 & 1.959 & 0.007 & 1 & \\
\hline $19052+2326$ & $\mathrm{BU}$ & $359 \mathrm{AB}$ & 2016.544 & 83.8 & 0.2 & 4.645 & 0.016 & 1 & \\
\hline $19058+0633$ & STF & $2446 \mathrm{AB}$ & 2016.642 & 152.9 & 0.2 & 9.449 & 0.032 & 1 & \\
\hline $19061+3335$ & TDT & 1223 & 2005.684 & 40.1 & 0.2 & 2.528 & 0.009 & 1 & A \\
\hline $19064-1043$ & HJ & 5090 & 2016.642 & 247.6 & 0.2 & 12.607 & 0.043 & 1 & \\
\hline $19066-0121$ & STF & $2447 \mathrm{AB}$ & 2016.642 & 343.1 & 0.2 & 14.000 & 0.048 & 1 & \\
\hline $19071+7204$ & STT & 369 & 2016.700 & 7.5 & 0.2 & 0.688 & 0.002 & 1 & \\
\hline $19080+1945$ & $\mathrm{STF}$ & 2460 & 2016.544 & 200.1 & 0.2 & 9.333 & 0.032 & 1 & \\
\hline $19082+1448$ & $\mathrm{ROE}$ & $125 \mathrm{AB}$ & 2016.544 & 268.0 & 0.2 & 7.862 & 0.027 & 1 & \\
\hline $19082+1448$ & $\mathrm{ROE}$ & $125 \mathrm{AC}$ & 2016.544 & 38.0 & 0.2 & 41.246 & 0.140 & 1 & \\
\hline $19086-1445$ & $\mathrm{~J}$ & 2687 & 2016.642 & 245.4 & 0.2 & 5.803 & 0.020 & 1 & \\
\hline 19087-0918 & A & 94 & 2016.642 & 317.1 & 0.2 & 2.361 & 0.008 & 1 & \\
\hline $19089+0104$ & $\mathrm{~J}$ & 2688 & 2016.642 & 129.9 & 0.2 & 4.283 & 0.015 & 1 & \\
\hline $19092+0325$ & STF & $2462 \mathrm{AB}$ & 2016.642 & 171.1 & 0.2 & 8.492 & 0.029 & 1 & \\
\hline $19092+0325$ & STF & $2462 \mathrm{AC}$ & 2016.642 & 234.9 & 0.2 & 11.447 & 0.039 & 1 & \\
\hline $19092+0325$ & STF & $2462 \mathrm{AD}$ & 2016.642 & 132.0 & 0.2 & 9.508 & 0.032 & 1 & \\
\hline $19092+0325$ & STF & $2462 \mathrm{BC}$ & 2016.642 & 279.4 & 0.2 & 10.965 & 0.037 & 1 & \\
\hline $19092+0325$ & STF & 2462 BD & 2016.642 & 70.3 & 0.2 & 6.158 & 0.021 & 1 & \\
\hline $19097+2112$ & $\mathrm{~J}$ & 2946 & 2016.544 & 57.6 & 0.2 & 4.198 & 0.014 & 1 & \\
\hline $19102+0841$ & STF & 2468 & 2016.642 & 257.7 & 0.2 & 7.591 & 0.026 & 1 & \\
\hline $19103+0647$ & GRV & 248 & 2016.642 & 299.5 & 0.2 & 16.819 & 0.057 & 1 & \\
\hline $19125+4447$ & WFC & 218 & 2016.702 & 352.1 & 0.2 & 9.502 & 0.032 & 1 & \\
\hline $19127+4746$ & HJ & 1380 & 2016.702 & 225.6 & 0.2 & 5.297 & 0.018 & 1 & \\
\hline $19127+4945$ & SMA & 85 & 2016.702 & 211.1 & 0.2 & 30.729 & 0.104 & 1 & \\
\hline $19132+5507$ & FOX & $26 \mathrm{AC}$ & 2016.702 & 344.8 & 0.2 & 17.385 & 0.059 & 1 & \\
\hline $19146+6733$ & MLB & 356 & 2016.700 & 20.2 & 0.2 & 4.064 & 0.014 & 1 & \\
\hline $19148+4756$ & A & 706 & 2016.702 & 74.2 & 0.2 & 1.602 & 0.005 & 1 & \\
\hline
\end{tabular}


Table 4-Continued

\begin{tabular}{|c|c|c|c|c|c|c|c|c|c|}
\hline $\begin{array}{l}\text { WDS Desig. } \\
\alpha, \delta(2000)\end{array}$ & & $\begin{array}{l}\text { iscoverer } \\
\text { signation }\end{array}$ & JY & $\begin{array}{c}\theta \\
(0)\end{array}$ & $\begin{array}{l}\sigma \theta \\
(0)\end{array}$ & $\begin{array}{c}\rho \\
\left({ }^{\prime \prime}\right)\end{array}$ & $\begin{array}{l}\sigma \rho \\
\left({ }^{\prime \prime}\right)\end{array}$ & $\mathrm{n}$ & Note \\
\hline $19148+6220$ & STI & 883 & 2016.700 & 87.0 & 0.2 & 14.333 & 0.049 & 1 & \\
\hline $19191+4801$ & HJ & 1386 & 2016.702 & 146.1 & 0.2 & 10.714 & 0.036 & 1 & \\
\hline $19193+4217$ & ES & $1562 \mathrm{AB}$ & 2016.702 & 324.9 & 0.2 & 6.159 & 0.021 & 1 & \\
\hline $19212+6433$ & $\mathrm{ES}$ & 194 & 2016.700 & 217.2 & 0.2 & 4.640 & 0.016 & 1 & \\
\hline $19213+5549$ & STF & 2516 & 2016.702 & 234.3 & 0.2 & 4.148 & 0.014 & 1 & \\
\hline $19218+1422$ & STF & 2506 & 2016.645 & 349.4 & 0.2 & 17.692 & 0.060 & 1 & \\
\hline $19221-0037$ & BVD & 130 & 2016.653 & 296.1 & 0.2 & 30.320 & 0.103 & 1 & \\
\hline $19224+1459$ & $\mathrm{~J}$ & 822 & 2016.645 & 350.5 & 0.2 & 3.143 & 0.011 & 1 & \\
\hline $19227-0708$ & STF & 2503 & 2016.653 & 284.8 & 0.2 & 2.490 & 0.008 & 1 & \\
\hline $19231-0013$ & $\mathrm{~J}$ & 1285 & 2016.653 & 330.6 & 0.2 & 3.833 & 0.013 & 1 & \\
\hline $19233+0931$ & STF & $2510 \mathrm{~A}, \mathrm{BC}$ & 2016.647 & 181.1 & 0.1 & 8.707 & 0.030 & 2 & \\
\hline $19237+2746$ & $A G$ & 378 & 2016.645 & 45.4 & 0.2 & 8.559 & 0.029 & 1 & \\
\hline $19249-1150$ & $\mathrm{HU}$ & $74 \mathrm{AC}$ & 2016.613 & 209.5 & 0.2 & 20.594 & 0.070 & 1 & \\
\hline $19259+2324$ & $\mathrm{POU}$ & 3823 & 2016.645 & 148.1 & 0.2 & 18.179 & 0.062 & 1 & \\
\hline $19265-1308$ & HDS & 2761 & 2016.653 & 123.2 & 0.2 & 12.358 & 0.042 & 1 & \\
\hline $19268+2110$ & STF & $2523 \mathrm{AB}$ & 2016.645 & 148.1 & 0.2 & 6.392 & 0.022 & 1 & \\
\hline $19270+7322$ & STF & $2550 \mathrm{AB}$ & 2016.700 & 252.1 & 0.2 & 1.929 & 0.007 & 1 & \\
\hline $19273-0044$ & SKF & 922 & 2016.653 & 270.3 & 0.2 & 32.107 & 0.109 & 1 & \\
\hline $19273+2040$ & STF & $2527 \mathrm{AC}$ & 2016.645 & 16.5 & 0.2 & 4.336 & 0.015 & 1 & \\
\hline $19279+0402$ & BAL & 2512 & 2016.653 & 334.7 & 0.2 & 18.272 & 0.062 & 1 & \\
\hline $19281+1619$ & $\mathrm{~J}$ & $771 \mathrm{AB}$ & 2016.645 & 93.0 & 0.2 & 3.587 & 0.012 & 1 & \\
\hline $19282-0932$ & STF & 2519 & 2016.653 & 123.9 & 0.2 & 11.737 & 0.040 & 1 & \\
\hline $19284+1204$ & $\mathrm{AG}$ & 382 & 2016.645 & 189.5 & 0.2 & 10.981 & 0.037 & 1 & \\
\hline $19284+2019$ & STF & 2530 & 2016.645 & 155.4 & 0.2 & 5.579 & 0.019 & 1 & \\
\hline $19290+0343$ & HJ & $2872 \mathrm{AB}$ & 2016.653 & 112.5 & 0.2 & 9.604 & 0.033 & 1 & \\
\hline $19290+0343$ & HJ & $2872 \mathrm{AC}$ & 2016.653 & 233.1 & 0.2 & 12.467 & 0.042 & 1 & \\
\hline $19291+0239$ & SRW & $9 \mathrm{AB}$ & 2016.653 & 259.5 & 0.2 & 29.638 & 0.101 & 1 & \\
\hline $19291+0239$ & SRW & $9 \mathrm{AC}$ & 2016.653 & 319.4 & 0.2 & 25.221 & 0.086 & 1 & \\
\hline $19293-1742$ & HJ & 5124 & 2016.656 & 274.0 & 0.2 & 4.829 & 0.016 & 1 & \\
\hline $19295+7816$ & GUI & $23 \mathrm{DE}$ & 2016.700 & 32.7 & 0.2 & 35.485 & 0.121 & 1 & \\
\hline $19295+7816$ & STF & $2571 \mathrm{AB}$ & 2016.700 & 18.9 & 0.2 & 11.190 & 0.038 & 1 & \\
\hline $19296+1800$ & $A G$ & 231 & 2016.645 & 240.8 & 0.2 & 4.415 & 0.015 & 1 & \\
\hline
\end{tabular}


Table 4-Continued

\begin{tabular}{|c|c|c|c|c|c|c|c|c|c|}
\hline $\begin{array}{l}\text { WDS Desig. } \\
\alpha, \delta(2000)\end{array}$ & $\begin{array}{l}\text { Dis } \\
\text { Desi }\end{array}$ & $\begin{array}{l}\text { scoverer } \\
\text { ignation }\end{array}$ & JY & $\begin{array}{c}\theta \\
(0)\end{array}$ & $\begin{array}{l}\sigma \theta \\
(0)\end{array}$ & $\begin{array}{c}\rho \\
\left({ }^{\prime \prime}\right)\end{array}$ & $\begin{array}{l}\sigma \rho \\
\left({ }^{\prime \prime}\right)\end{array}$ & $\mathrm{n}$ & Note \\
\hline $19299+1241$ & BRT & 1318 & 2016.645 & 83.8 & 0.2 & 5.886 & 0.020 & 1 & \\
\hline $19300+0916$ & HJ & 888 & 2016.645 & 230.6 & 0.2 & 10.946 & 0.037 & 1 & \\
\hline $19303+0419$ & BAL & 2943 & 2016.653 & 288.6 & 0.2 & 12.252 & 0.042 & 1 & \\
\hline $19307+2758$ & STFA & $43 \mathrm{AB}$ & 2016.645 & 54.1 & 0.2 & 34.719 & 0.118 & 1 & \\
\hline 19336-1837 & HJ & $5128 \mathrm{AB}$ & 2016.656 & 111.1 & 0.2 & 21.311 & 0.072 & 1 & \\
\hline $19336-1837$ & HJ & $5128 \mathrm{BC}$ & 2016.656 & 127.9 & 0.2 & 4.273 & 0.015 & 1 & \\
\hline $19340-1616$ & HJ & 2880 & 2016.656 & 150.2 & 0.2 & 4.119 & 0.014 & 1 & \\
\hline $19340+6017$ & STF & 2554 & 2016.700 & 194.6 & 0.2 & 18.637 & 0.063 & 1 & \\
\hline $19342-1245$ & $\mathrm{~J}$ & 1762 & 2016.656 & 171.3 & 0.2 & 5.434 & 0.018 & 1 & \\
\hline $19345-0129$ & HJ & 2882 & 2016.656 & 131.7 & 0.2 & 9.973 & 0.034 & 1 & \\
\hline $19354-0249$ & BRT & 493 & 2016.656 & 112.9 & 0.2 & 4.533 & 0.015 & 1 & \\
\hline $19371-1607$ & BRT & 635 & 2016.656 & 245.0 & 0.2 & 5.000 & 0.017 & 1 & \\
\hline $19374-0032$ & $\mathrm{~J}$ & 1681 & 2016.656 & 4.2 & 0.2 & 6.250 & 0.021 & 1 & \\
\hline $19380+3354$ & STT & $379 \mathrm{AB}$ & 2016.727 & 83.9 & 0.2 & 25.120 & 0.085 & 1 & \\
\hline $19397-1115$ & WFC & 255 & 2016.730 & 176.8 & 0.2 & 12.833 & 0.044 & 1 & \\
\hline $19397-1827$ & HJ & 2884 & 2016.730 & 118.8 & 0.2 & 18.816 & 0.064 & 1 & \\
\hline $19397+3315$ & GYL & 19 & 2016.727 & 11.6 & 0.2 & 22.610 & 0.077 & 1 & \\
\hline $19399-1703$ & ARN & 53 & 2016.730 & 253.9 & 0.2 & 26.755 & 0.091 & 1 & \\
\hline $19407-1618$ & HJ & $599 \mathrm{AC}$ & 2016.730 & 41.8 & 0.2 & 45.611 & 0.155 & 1 & \\
\hline $19409+3559$ & ALI & 157 & 2016.659 & 198.1 & 0.2 & 11.408 & 0.039 & 1 & \\
\hline $19412+7208$ & FOX & 245 & 2016.700 & 68.5 & 0.2 & 5.466 & 0.019 & 1 & \\
\hline $19413-1325$ & HJ & 2887 & 2016.730 & 236.4 & 0.2 & 11.599 & 0.039 & 1 & \\
\hline $19414+3313$ & HJ & $1430 \mathrm{AB}$ & 2016.727 & 154.0 & 0.2 & 17.402 & 0.059 & 1 & \\
\hline $19418+3611$ & ALI & 158 & 2016.659 & 178.6 & 0.2 & 15.224 & 0.052 & 1 & \\
\hline $19422+0919$ & $\mathrm{~J}$ & $1183 \mathrm{AC}$ & 2016.784 & 58.7 & 0.2 & 17.513 & 0.060 & 1 & \\
\hline $19428+0823$ & STF & $2562 \mathrm{AB}$ & 2016.730 & 251.6 & 0.2 & 27.330 & 0.093 & 1 & \\
\hline $19430+3433$ & SEI & 663 & 2016.659 & 296.6 & 0.2 & 28.156 & 0.096 & 1 & \\
\hline $19435+3433$ & SEI & 669 & 2016.659 & 47.4 & 0.2 & 17.338 & 0.059 & 1 & \\
\hline $19435+3450$ & $\mathrm{AG}$ & $236 \mathrm{AB}$ & 2016.659 & 148.1 & 0.2 & 4.244 & 0.014 & 1 & \\
\hline $19437+3225$ & HJ & 1433 & 2016.727 & 299.1 & 0.2 & 16.950 & 0.058 & 1 & \\
\hline $19443+3401$ & GYL & $20 \mathrm{AB}$ & 2016.659 & 111.6 & 0.2 & 25.260 & 0.086 & 1 & \\
\hline $19443+3401$ & TOB & $164 \mathrm{AC}$ & 2016.659 & 315.8 & 0.2 & 21.272 & 0.072 & 1 & \\
\hline
\end{tabular}


Table 4-Continued

\begin{tabular}{|c|c|c|c|c|c|c|c|c|c|}
\hline $\begin{array}{l}\text { WDS Desig. } \\
\alpha, \delta(2000)\end{array}$ & $\begin{array}{l}\text { Dis } \\
\text { Desi }\end{array}$ & $\begin{array}{l}\text { coverer } \\
\text { ignation }\end{array}$ & JY & $\begin{array}{c}\theta \\
(0)\end{array}$ & $\begin{array}{l}\sigma \theta \\
(0)\end{array}$ & $\begin{array}{c}\rho \\
\left({ }^{\prime \prime}\right)\end{array}$ & $\begin{array}{l}\sigma \rho \\
(")\end{array}$ & $\mathrm{n}$ & Note \\
\hline 19444-1102 & CLS & 1 & 2016.730 & 256.4 & 0.2 & 1.787 & 0.006 & 1 & \\
\hline $19448-2029$ & DON & $965 \mathrm{AC}$ & 2016.757 & 208.3 & 0.2 & 4.811 & 0.016 & 1 & \\
\hline $19448-2029$ & HJ & $2890 \mathrm{AD}$ & 2016.757 & 280.2 & 0.2 & 30.687 & 0.104 & 1 & \\
\hline $19453+0523$ & $\mathrm{~J}$ & $2998 \mathrm{AC}$ & 2016.730 & 192.5 & 0.2 & 6.286 & 0.021 & 1 & \\
\hline 19453-1314 & STF & 2565 & 2016.730 & 40.5 & 0.2 & 5.503 & 0.019 & 1 & \\
\hline $19457+3605$ & STF & $2578 \mathrm{AB}$ & 2016.659 & 124.7 & 0.2 & 14.941 & 0.051 & 1 & \\
\hline $19457+3612$ & ALI & $161 \mathrm{AB}$ & 2016.659 & 16.7 & 0.2 & 8.547 & 0.029 & 1 & \\
\hline $19458+3223$ & HDS & 2807 & 2016.727 & 287.1 & 0.2 & 22.143 & 0.075 & 1 & \\
\hline $19458+3341$ & SMA & 91 & 2016.659 & 323.3 & 0.2 & 6.400 & 0.022 & 1 & \\
\hline $19459+3501$ & H 5 & $137 \mathrm{AB}$ & 2016.659 & 25.0 & 0.2 & 38.713 & 0.132 & 1 & \\
\hline $19467-1129$ & HDS & 2811 & 2016.757 & 201.0 & 0.2 & 16.245 & 0.055 & 1 & \\
\hline $19468+1839$ & $\mathrm{OL}$ & 72 & 2016.648 & 9.2 & 0.2 & 7.118 & 0.024 & 1 & \\
\hline $19471+1509$ & HJ & 1436 & 2016.648 & 305.5 & 0.2 & 8.430 & 0.029 & 1 & \\
\hline $19474+3926$ & MLB & 980 & 2016.683 & 31.2 & 0.2 & 6.394 & 0.022 & 1 & \\
\hline $19475+0615$ & $\mathrm{~J}$ & $2186 \mathrm{AC}$ & 2005.687 & 334.0 & 0.2 & 6.963 & 0.024 & 1 & A \\
\hline $19475+0615$ & $\mathrm{~J}$ & $2186 \mathrm{CB}$ & 2005.687 & 155.3 & 0.2 & 11.028 & 0.037 & 1 & $\mathrm{~A}$ \\
\hline $19480-1434$ & HDS & 2814 & 2016.757 & 138.7 & 0.2 & 18.311 & 0.062 & 1 & \\
\hline $19480+3959$ & ALI & 1120 & 2016.683 & 172.8 & 0.2 & 13.392 & 0.046 & 1 & \\
\hline $19483+3710$ & STT & 386 & 2016.683 & 69.8 & 0.2 & 0.921 & 0.003 & 1 & \\
\hline $19484+2212$ & STF & 2584 & 2016.760 & 293.3 & 0.2 & 1.943 & 0.007 & 1 & \\
\hline $19485+1958$ & $\mathrm{~J}$ & 1865 & 2016.648 & 22.0 & 0.2 & 6.112 & 0.021 & 1 & \\
\hline $19487-0137$ & $\mathrm{~J}$ & 1687 & 2016.648 & 176.6 & 0.2 & 4.935 & 0.017 & 1 & \\
\hline $19487+1149$ & STF & $2583 \mathrm{AB}$ & 2016.648 & 104.9 & 0.2 & 1.453 & 0.005 & 1 & \\
\hline $19490+1711$ & BRT & 1328 & 2016.648 & 130.1 & 0.2 & 4.599 & 0.016 & 1 & \\
\hline $19492+1428$ & $\mathrm{AG}$ & 392 & 2016.648 & 152.6 & 0.2 & 3.568 & 0.012 & 1 & \\
\hline $19493+2202$ & CHE & 152 & 2016.760 & 253.4 & 0.2 & 27.469 & 0.093 & 1 & \\
\hline $19495+4123$ & ES & 1671 & 2016.683 & 62.4 & 0.2 & 4.209 & 0.014 & 1 & \\
\hline $19498+1844$ & $\mathrm{~J}$ & 3014 & 2016.648 & 346.6 & 0.2 & 6.443 & 0.022 & 1 & \\
\hline $19504-1114$ & $\mathrm{UC}$ & 4035 & 2016.757 & 322.5 & 0.2 & 14.192 & 0.048 & 1 & \\
\hline $19504-1456$ & HDS & 2824 & 2016.757 & 332.8 & 0.2 & 14.004 & 0.048 & 1 & \\
\hline $19505+2030$ & $\mathrm{~J}$ & 3018 & 2016.760 & 164.7 & 0.2 & 6.889 & 0.023 & 1 & \\
\hline 19506-1917 & HJ & 2900 & 2016.757 & 63.9 & 0.2 & 19.389 & 0.066 & 1 & \\
\hline
\end{tabular}


Table 4-Continued

\begin{tabular}{|c|c|c|c|c|c|c|c|c|c|}
\hline $\begin{array}{l}\text { WDS Desig. } \\
\alpha, \delta(2000)\end{array}$ & & $\begin{array}{l}\text { iscoverer } \\
\text { signation }\end{array}$ & JY & $\begin{array}{c}\theta \\
(0)\end{array}$ & $\begin{array}{l}\sigma \theta \\
(0)\end{array}$ & $\begin{array}{c}\rho \\
\left({ }^{\prime \prime}\right)\end{array}$ & $\begin{array}{l}\sigma \rho \\
\left({ }^{\prime \prime}\right)\end{array}$ & $\mathrm{n}$ & Note \\
\hline $19507+2018$ & TVB & 5 & 2016.760 & 239.6 & 0.2 & 9.268 & 0.032 & 1 & \\
\hline $19510+3650$ & SEI & 698 & 2016.683 & 20.1 & 0.2 & 17.275 & 0.059 & 1 & \\
\hline $19513+1430$ & HJ & 1442 & 2016.648 & 275.9 & 0.2 & 7.629 & 0.026 & 1 & \\
\hline $19514-0215$ & BAL & 254 & 2016.648 & 5.6 & 0.2 & 5.060 & 0.017 & 1 & \\
\hline $19515+2332$ & $\mathrm{BU}$ & 978 & 2016.760 & 58.0 & 0.2 & 1.022 & 0.003 & 1 & \\
\hline $19516+2009$ & $\mathrm{HU}$ & 350 & 2016.760 & 45.4 & 0.2 & 3.400 & 0.012 & 1 & \\
\hline $19517+5339$ & ES & 2683 & 2016.683 & 318.7 & 0.2 & 9.988 & 0.034 & 1 & \\
\hline $19519-0122$ & $\mathrm{~J}$ & 1868 & 2016.648 & 293.5 & 0.2 & 6.785 & 0.023 & 1 & \\
\hline $19519+2032$ & $\mathrm{COU}$ & $824 \mathrm{AB}$ & 2016.760 & 81.6 & 0.2 & 4.210 & 0.014 & 1 & \\
\hline $19519+2032$ & $\mathrm{COU}$ & $824 \mathrm{AC}$ & 2016.760 & 291.7 & 0.2 & 20.605 & 0.070 & 1 & \\
\hline $19521+0041$ & $\mathrm{~J}$ & 828 & 2016.648 & 194.8 & 0.2 & 2.192 & 0.007 & 1 & \\
\hline $19521+3600$ & ALI & 164 & 2016.683 & 276.3 & 0.2 & 10.510 & 0.036 & 1 & \\
\hline $19522+3139$ & ES & 356 & 2016.727 & 343.7 & 0.2 & 6.154 & 0.021 & 1 & \\
\hline $19523+4545$ & $\mathrm{KU}$ & 125 & 2016.683 & 265.0 & 0.2 & 54.982 & 0.187 & 1 & \\
\hline $19525+0039$ & STF & 2589 & 2016.648 & 294.1 & 0.2 & 4.992 & 0.017 & 1 & \\
\hline $19531+5430$ & STI & $2471 \mathrm{AB}$ & 2016.683 & 113.2 & 0.2 & 11.708 & 0.040 & 1 & \\
\hline $19531+5430$ & STI & $2471 \mathrm{CD}$ & 2016.683 & 299.5 & 0.2 & 10.493 & 0.036 & 1 & \\
\hline $19536+4915$ & TOB & 161 & 2016.683 & 220.6 & 0.2 & 22.315 & 0.076 & 1 & \\
\hline $19541+4236$ & $\mathrm{ROE}$ & 145 & 2016.683 & 253.7 & 0.2 & 11.723 & 0.040 & 1 & \\
\hline $19546-0814$ & STF & 2594 & 2016.648 & 170.0 & 0.2 & 35.881 & 0.122 & 1 & \\
\hline $19547+3453$ & SEI & $723 \mathrm{AB}$ & 2005.745 & 40.8 & 0.2 & 11.794 & 0.040 & 1 & A \\
\hline $19548-1223$ & HJ & 602 & 2016.757 & 309.3 & 0.2 & 6.714 & 0.023 & 1 & \\
\hline $19563-1321$ & STF & 2602 & 2016.656 & 147.6 & 0.2 & 12.320 & 0.042 & 1 & \\
\hline $19565+1434$ & $\mathrm{~J}$ & 2564 & 2016.664 & 22.1 & 0.2 & 4.827 & 0.016 & 1 & \\
\hline $19567-0113$ & BAL & $606 \mathrm{AB}$ & 2016.656 & 146.8 & 0.2 & 10.314 & 0.035 & 1 & \\
\hline $19567-0113$ & BAL & $606 \mathrm{AC}$ & 2016.656 & 139.6 & 0.2 & 26.647 & 0.091 & 1 & \\
\hline $19567-0113$ & BAL & $606 \mathrm{BC}$ & 2016.656 & 134.6 & 0.2 & 16.344 & 0.056 & 1 & \\
\hline $19567-0737$ & RST & 4646 & 2016.656 & 195.8 & 0.2 & 4.528 & 0.015 & 1 & \\
\hline $19568+0155$ & STF & $2601 \mathrm{AB}, \mathrm{C}$ & 2016.656 & 162.0 & 0.2 & 6.969 & 0.024 & 1 & \\
\hline $19568+1935$ & $\mathrm{~J}$ & $3036 \mathrm{AB}$ & 2016.664 & 256.8 & 0.2 & 6.977 & 0.024 & 1 & \\
\hline $19568+1935$ & TOB & $304 \mathrm{AC}$ & 2016.664 & 324.6 & 0.2 & 19.354 & 0.066 & 1 & \\
\hline $19573+0256$ & $\mathrm{~J}$ & 2600 & 2016.656 & 56.5 & 0.2 & 1.529 & 0.005 & 1 & \\
\hline
\end{tabular}


Table 4-Continued

\begin{tabular}{|c|c|c|c|c|c|c|c|c|c|}
\hline $\begin{array}{l}\text { WDS Desig. } \\
\alpha, \delta(2000)\end{array}$ & $\begin{array}{l}\text { Dis } \\
\text { Desi }\end{array}$ & $\begin{array}{l}\text { scoverer } \\
\text { ignation }\end{array}$ & JY & $\begin{array}{c}\theta \\
(0)\end{array}$ & $\begin{array}{l}\sigma \theta \\
(\circ)\end{array}$ & $\begin{array}{c}\rho \\
\left({ }^{\prime \prime}\right)\end{array}$ & $\begin{array}{l}\sigma \rho \\
\left({ }^{\prime \prime}\right)\end{array}$ & $\mathrm{n}$ & Note \\
\hline $19575+1408$ & $\mathrm{~A}$ & 1662 & 2016.664 & 194.1 & 0.2 & 0.396 & 0.001 & 1 & \\
\hline $19575+2018$ & $\mathrm{BU}$ & $425 \mathrm{AB}$ & 2016.664 & 239.7 & 0.2 & 1.375 & 0.005 & 1 & \\
\hline $19576+0425$ & BAL & 2957 & 2016.664 & 191.4 & 0.2 & 13.408 & 0.046 & 1 & \\
\hline $19576+1524$ & $\mathrm{~A}$ & $1663 \mathrm{AB}$ & 2016.664 & 237.4 & 0.2 & 1.298 & 0.004 & 1 & \\
\hline $19584-0214$ & $\mathrm{AC}$ & 12 & 2016.656 & 298.5 & 0.2 & 1.552 & 0.005 & 1 & \\
\hline $19585+1627$ & ARY & 60 & 2016.664 & 123.7 & 0.2 & 42.149 & 0.143 & 1 & \\
\hline $19591+1437$ & $\mathrm{~J}$ & $3218 \mathrm{AB}$ & 2016.664 & 46.8 & 0.2 & 4.234 & 0.014 & 1 & \\
\hline $19593+2215$ & WFC & 262 & 2016.664 & 31.2 & 0.2 & 13.239 & 0.045 & 1 & \\
\hline $19596-1737$ & HJ & 2912 & 2016.735 & 134.5 & 0.2 & 16.678 & 0.057 & 1 & \\
\hline $19597-0358$ & $\mathrm{~J}$ & 2565 & 2016.656 & 298.7 & 0.2 & 4.387 & 0.015 & 1 & \\
\hline $20017-0012$ & H 1 & $93 \mathrm{AB}$ & 2016.656 & 298.3 & 0.2 & 1.824 & 0.006 & 1 & \\
\hline $20017+5806$ & $\mathrm{AG}$ & 395 & 2016.702 & 357.0 & 0.2 & 4.319 & 0.015 & 1 & \\
\hline $20018+0311$ & HJ & 2920 & 2016.735 & 165.3 & 0.2 & 7.413 & 0.025 & 1 & \\
\hline $20018-0354$ & $\mathrm{~J}$ & 154 & 2016.735 & 59.2 & 0.2 & 4.262 & 0.014 & 1 & \\
\hline $20021+5439$ & $\mathrm{BU}$ & $426 \mathrm{AB}$ & 2016.702 & 306.6 & 0.2 & 5.858 & 0.020 & 1 & \\
\hline $20022+0049$ & BAL & 1211 & 2016.735 & 80.5 & 0.2 & 12.090 & 0.041 & 1 & \\
\hline $20023+0512$ & $\mathrm{~J}$ & $1292 \mathrm{AB}$ & 2016.735 & 93.1 & 0.2 & 4.438 & 0.015 & 1 & \\
\hline $20033+3807$ & SEI & 825 & 2016.702 & 155.4 & 0.2 & 7.191 & 0.024 & 1 & \\
\hline $20035+3924$ & SEI & $828 \mathrm{AB}$ & 2016.702 & 85.3 & 0.2 & 19.830 & 0.067 & 1 & \\
\hline $20035+3924$ & SEI & $829 \mathrm{AC}$ & 2016.702 & 262.5 & 0.2 & 22.711 & 0.077 & 1 & \\
\hline $20037+3820$ & HJ & 1470 & 2016.702 & 340.6 & 0.2 & 29.261 & 0.099 & 1 & \\
\hline $20041+0404$ & $\mathrm{~J}$ & 2296 & 2016.735 & 284.1 & 0.2 & 8.772 & 0.030 & 1 & \\
\hline $20044+3820$ & TOR & 20 & 2016.702 & 158.7 & 0.2 & 17.304 & 0.059 & 1 & \\
\hline $20056+6342$ & STF & $2642 \mathrm{AB}$ & 2016.702 & 191.1 & 0.2 & 1.781 & 0.006 & 1 & \\
\hline $20057+5925$ & HJ & $2934 \mathrm{AB}$ & 2016.702 & 310.9 & 0.2 & 9.746 & 0.033 & 1 & \\
\hline $20057+5925$ & HJ & $2934 \mathrm{CA}$ & 2016.702 & 263.8 & 0.2 & 36.568 & 0.124 & 1 & \\
\hline $20058+3520$ & SEI & 851 & 2016.659 & 161.3 & 0.2 & 27.249 & 0.093 & 1 & \\
\hline $20059+3542$ & SEI & 854 & 2016.659 & 108.4 & 0.2 & 13.041 & 0.044 & 1 & \\
\hline $20060+3547$ & SHJ & $314 \mathrm{AD}$ & 2016.659 & 299.3 & 0.2 & 11.306 & 0.038 & 1 & \\
\hline $20060+3547$ & SHJ & $314 \mathrm{AF}$ & 2016.659 & 28.0 & 0.2 & 36.038 & 0.123 & 1 & \\
\hline $20063+3538$ & SEI & $871 \mathrm{AB}$ & 2016.659 & 172.5 & 0.2 & 11.531 & 0.039 & 1 & \\
\hline $20063+3538$ & SEI & $872 \mathrm{AC}$ & 2016.659 & 56.4 & 0.0 & 29.058 & 0.039 & 2 & \\
\hline
\end{tabular}


Table 4-Continued

\begin{tabular}{|c|c|c|c|c|c|c|c|c|c|}
\hline $\begin{array}{l}\text { WDS Desig. } \\
\alpha, \delta(2000)\end{array}$ & $\begin{array}{l}\text { Dis } \\
\text { Desi }\end{array}$ & $\begin{array}{l}\text { coverer } \\
\text { ignation }\end{array}$ & JY & $\begin{array}{l}\theta \\
(0)\end{array}$ & $\begin{array}{l}\sigma \theta \\
(0)\end{array}$ & $\begin{array}{c}\rho \\
\left({ }^{\prime \prime}\right)\end{array}$ & $\begin{array}{l}\sigma \rho \\
\left({ }^{\prime \prime}\right)\end{array}$ & $\mathrm{n}$ & Note \\
\hline 20064-1322 & HLD & $155 \mathrm{AB}$ & 2016.735 & 276.0 & 0.2 & 1.830 & 0.006 & 1 & \\
\hline 20064-1322 & HLD & $155 \mathrm{AC}$ & 2016.735 & 240.1 & 0.2 & 29.854 & 0.102 & 1 & \\
\hline $20066+0207$ & HJ & 902 & 2016.735 & 17.6 & 0.2 & 6.952 & 0.024 & 1 & \\
\hline $20068-1256$ & STF & 2625 & 2016.735 & 4.5 & 0.2 & 12.125 & 0.041 & 1 & \\
\hline $20069+3546$ & SEI & 885 & 2016.659 & 285.3 & 0.2 & 14.076 & 0.048 & 1 & \\
\hline $20073+6143$ & $\mathrm{ES}$ & $1852 \mathrm{AB}$ & 2016.702 & 156.9 & 0.2 & 6.400 & 0.022 & 1 & \\
\hline $20077+5908$ & HJ & 2936 & 2016.702 & 251.2 & 0.2 & 12.724 & 0.043 & 1 & \\
\hline $20084+3808$ & HJ & 606 & 2016.646 & 228.2 & 0.2 & 44.307 & 0.151 & 1 & \\
\hline $20090+1808$ & HJ & $2932 \mathrm{AB}$ & 2016.814 & 165.4 & 0.2 & 13.491 & 0.046 & 1 & \\
\hline $20099+2055$ & STF & $2637 \mathrm{AB}$ & 2016.814 & 331.0 & 0.2 & 11.646 & 0.040 & 1 & \\
\hline $20102+0204$ & HJ & 2933 & 2016.814 & 27.0 & 0.2 & 21.714 & 0.074 & 1 & \\
\hline $20119+0348$ & STF & 2641 & 2016.814 & 168.4 & 0.2 & 20.719 & 0.070 & 1 & \\
\hline $20123+1856$ & $\mathrm{~J}$ & 3056 & 2016.814 & 256.2 & 0.2 & 6.262 & 0.021 & 1 & \\
\hline $20123+2215$ & $\mathrm{~J}$ & 3057 & 2016.814 & 104.2 & 0.2 & 6.703 & 0.023 & 1 & \\
\hline $20125+1122$ & $\mathrm{AG}$ & 402 & 2016.814 & 261.2 & 0.2 & 20.008 & 0.068 & 1 & \\
\hline $20126+0052$ & STF & 2644 & 2016.757 & 206.0 & 0.2 & 2.619 & 0.009 & 1 & \\
\hline $20128+2312$ & $\mathrm{POU}$ & 4276 & 2016.814 & 96.5 & 0.2 & 9.948 & 0.034 & 1 & \\
\hline $20132+0256$ & CRI & 26 & 2016.757 & 123.9 & 0.2 & 32.620 & 0.111 & 1 & \\
\hline $20134+0201$ & BAL & 1549 & 2016.757 & 228.3 & 0.2 & 17.168 & 0.058 & 1 & \\
\hline $20149+0315$ & BAL & 2021 & 2016.757 & 343.9 & 0.2 & 4.458 & 0.015 & 1 & \\
\hline $20152-0330$ & $\mathrm{STF}$ & 2654 & 2016.757 & 233.3 & 0.2 & 14.434 & 0.049 & 1 & \\
\hline 20157-1849 & HJ & $2940 \mathrm{AC}$ & 2016.757 & 76.9 & 0.2 & 20.767 & 0.071 & 1 & \\
\hline $20166+0405$ & $\mathrm{~J}$ & 1641 & 2016.757 & 215.7 & 0.2 & 7.224 & 0.025 & 1 & \\
\hline $20166+0507$ & $\mathrm{BU}$ & 59 & 2016.757 & 101.0 & 0.2 & 8.361 & 0.028 & 1 & \\
\hline $20169+1303$ & $\mathrm{~J}$ & $1879 \mathrm{AB}$ & 2016.757 & 233.4 & 0.2 & 11.369 & 0.039 & 1 & \\
\hline $20169+1303$ & J & $1879 \mathrm{AC}$ & 2016.757 & 272.2 & 0.2 & 13.737 & 0.047 & 1 & \\
\hline $20170-1228$ & DOO & $84 \mathrm{AC}$ & 2016.757 & 10.6 & 0.2 & 25.891 & 0.088 & 1 & \\
\hline $20170-1228$ & HJ & $2943 \mathrm{AB}$ & 2016.757 & 113.0 & 0.2 & 9.913 & 0.034 & 1 & \\
\hline $20187+1551$ & CHE & $297 \mathrm{AB}$ & 2016.850 & 6.4 & 0.2 & 35.050 & 0.119 & 1 & \\
\hline $20187+1551$ & CHE & $298 \mathrm{AC}$ & 2016.850 & 318.1 & 0.2 & 16.763 & 0.057 & 1 & \\
\hline $20187+1551$ & CHE & $298 \mathrm{BC}$ & 2016.850 & 214.0 & 0.2 & 26.963 & 0.092 & 1 & \\
\hline $20194+1534$ & TOB & 310 & 2016.850 & 239.5 & 0.2 & 16.338 & 0.056 & 1 & \\
\hline
\end{tabular}


Table 4-Continued

\begin{tabular}{|c|c|c|c|c|c|c|c|c|c|}
\hline $\begin{array}{l}\text { WDS Desig. } \\
\alpha, \delta(2000)\end{array}$ & $\begin{array}{l}\text { Dis } \\
\text { Desi }\end{array}$ & $\begin{array}{l}\text { coverer } \\
\text { ignation }\end{array}$ & JY & $\begin{array}{c}\theta \\
(\circ)\end{array}$ & $\begin{array}{l}\sigma \theta \\
(0)\end{array}$ & $\begin{array}{c}\rho \\
\left({ }^{\prime \prime}\right)\end{array}$ & $\begin{array}{l}\sigma \rho \\
\left({ }^{\prime \prime}\right)\end{array}$ & $\mathrm{n}$ & Note \\
\hline $20208-0745$ & SCJ & 25 & 2016.875 & 215.9 & 0.2 & 2.763 & 0.009 & 1 & \\
\hline $20211+0837$ & HJ & 2953 & 2016.850 & 260.8 & 0.2 & 26.321 & 0.089 & 1 & \\
\hline $20220-1854$ & DON & 1119 & 2016.653 & 196.5 & 0.2 & 5.294 & 0.018 & 1 & \\
\hline $20222-1647$ & $\mathrm{H} \mathrm{N}$ & 138 & 2016.875 & 327.5 & 0.2 & 3.162 & 0.011 & 1 & \\
\hline $20224-1841$ & B & 479 & 2016.875 & 116.9 & 0.2 & 2.303 & 0.008 & 1 & \\
\hline $20247+1438$ & HJ & 1507 & 2016.850 & 64.4 & 0.2 & 10.384 & 0.035 & 1 & \\
\hline $20247+1443$ & HJ & $1508 \mathrm{AB}$ & 2016.875 & 59.0 & 0.2 & 13.756 & 0.047 & 1 & \\
\hline $20247+1443$ & VLM & $4 \mathrm{AC}$ & 2016.875 & 153.1 & 0.2 & 17.426 & 0.059 & 1 & \\
\hline $20250+1016$ & HJ & 1509 & 2016.850 & 180.7 & 0.2 & 19.021 & 0.065 & 1 & \\
\hline $20260+1212$ & $\mathrm{~J}$ & 1704 & 2016.850 & 202.9 & 0.2 & 6.025 & 0.020 & 1 & \\
\hline $20263+1445$ & $\mathrm{~J}$ & 3085 & 2016.850 & 206.8 & 0.2 & 6.029 & 0.020 & 1 & \\
\hline $20275-0206$ & S & $749 \mathrm{AB}$ & 2016.645 & 188.4 & 0.2 & 60.028 & 0.204 & 1 & \\
\hline $20279+0958$ & AG & $256 \mathrm{AB}$ & 2016.645 & 351.7 & 0.2 & 5.099 & 0.017 & 1 & \\
\hline $20283-1348$ & $\mathrm{UC}$ & 4194 & 2016.653 & 337.5 & 0.2 & 31.030 & 0.106 & 1 & \\
\hline 20284-1309 & STF & 2683 & 2016.653 & 66.8 & 0.2 & 22.935 & 0.078 & 1 & \\
\hline $20284+1713$ & HJ & 2969 & 2016.645 & 169.7 & 0.2 & 5.881 & 0.020 & 1 & \\
\hline $20286+2404$ & $\mathrm{KU}$ & $59 \mathrm{AB}$ & 2016.825 & 141.9 & 0.2 & 32.933 & 0.112 & 1 & \\
\hline $20286+2404$ & $\mathrm{KU}$ & $59 \mathrm{BC}$ & 2016.825 & 316.7 & 0.2 & 4.307 & 0.015 & 1 & \\
\hline $20299-1835$ & SHJ & 324 & 2016.653 & 238.6 & 0.2 & 22.078 & 0.075 & 1 & \\
\hline $20306+1404$ & $\mathrm{AG}$ & 408 & 2016.645 & 285.1 & 0.2 & 4.952 & 0.017 & 1 & \\
\hline $20308+1347$ & $\mathrm{STF}$ & 2688 & 2016.645 & 175.2 & 0.2 & 7.862 & 0.027 & 1 & \\
\hline $20309+1126$ & LAU & 4 & 2016.645 & 272.1 & 0.2 & 28.109 & 0.096 & 1 & \\
\hline $20310+2007$ & HJ & 2974 & 2016.825 & 297.2 & 0.2 & 14.414 & 0.049 & 1 & \\
\hline $20315+0444$ & BAL & 2966 & 2016.645 & 117.9 & 0.2 & 10.449 & 0.036 & 1 & \\
\hline $20315+1448$ & $\mathrm{~J}$ & 1884 & 2016.645 & 132.5 & 0.2 & 8.751 & 0.030 & 1 & \\
\hline $20320+2056$ & DAM & 216 & 2016.825 & 76.2 & 0.2 & 11.842 & 0.040 & 1 & \\
\hline $20328-1333$ & $\mathrm{HO}$ & $133 \mathrm{AB}$ & 2016.653 & 173.7 & 0.2 & 1.295 & 0.004 & 1 & \\
\hline $20328-1343$ & $\mathrm{HO}$ & 132 & 2016.653 & 206.8 & 0.2 & 7.007 & 0.024 & 1 & \\
\hline $20329+1803$ & HJ & 2977 & 2016.645 & 316.7 & 0.2 & 19.684 & 0.067 & 1 & \\
\hline $20339+2227$ & GRV & 353 & 2016.825 & 82.9 & 0.2 & 19.735 & 0.067 & 1 & \\
\hline $20347+3230$ & $\mathrm{STF}$ & 2700 & 2016.825 & 284.7 & 0.2 & 24.163 & 0.082 & 1 & \\
\hline $20354+2413$ & $\mathrm{POU}$ & 4704 & 2016.817 & 260.0 & 0.2 & 14.479 & 0.049 & 1 & \\
\hline
\end{tabular}


Table 4-Continued

\begin{tabular}{|c|c|c|c|c|c|c|c|c|c|}
\hline $\begin{array}{l}\text { WDS Desig. } \\
\alpha, \delta(2000)\end{array}$ & & $\begin{array}{l}\text { iscoverer } \\
\text { signation }\end{array}$ & JY & $\begin{array}{c}\theta \\
(0)\end{array}$ & $\begin{array}{l}\sigma \theta \\
(0)\end{array}$ & $\begin{array}{c}\rho \\
\left({ }^{\prime \prime}\right)\end{array}$ & $\begin{array}{l}\sigma \rho \\
\left({ }^{\prime \prime}\right)\end{array}$ & $\mathrm{n}$ & Note \\
\hline 20363-1519 & HJ & 1537 & 2016.875 & 22.9 & 0.2 & 3.477 & 0.012 & 1 & \\
\hline $20366+1027$ & $\mathrm{AG}$ & 258 & 2016.817 & 9.6 & 0.2 & 4.171 & 0.014 & 1 & \\
\hline $20370+0452$ & SMA & 118 & 2016.817 & 125.9 & 0.2 & 19.070 & 0.065 & 1 & \\
\hline $20370+1203$ & STF & 2701 & 2016.817 & 221.4 & 0.2 & 2.084 & 0.007 & 1 & \\
\hline $20371+6324$ & DOB & 14 & 2016.700 & 239.2 & 0.2 & 4.824 & 0.016 & 1 & \\
\hline $20378+3224$ & ARY & 48 & 2016.817 & 41.2 & 0.2 & 53.284 & 0.181 & 1 & \\
\hline $20382+2511$ & $\mathrm{AG}$ & 260 & 2016.817 & 219.4 & 0.2 & 9.673 & 0.033 & 1 & \\
\hline $20382+3109$ & $\mathrm{AG}$ & 261 & 2016.817 & 155.0 & 0.2 & 4.314 & 0.015 & 1 & \\
\hline 20390-0035 & BVD & 131 & 2016.817 & 133.9 & 0.2 & 30.299 & 0.103 & 1 & \\
\hline $20392+1059$ & SCJ & $27 \mathrm{AB}$ & 2016.817 & 262.8 & 0.2 & 6.226 & 0.021 & 1 & \\
\hline $20396+2018$ & WFC & 257 & 2016.817 & 317.3 & 0.2 & 6.764 & 0.023 & 1 & \\
\hline $20397+6325$ & DOB & 15 & 2016.700 & 90.2 & 0.2 & 3.118 & 0.011 & 1 & \\
\hline $20398-1827$ & HJ & $2983 \mathrm{AB}, \mathrm{C}$ & 2016.875 & 175.1 & 0.2 & 15.486 & 0.053 & 1 & \\
\hline $20404+2425$ & $\mathrm{POU}$ & 4847 & 2016.817 & 55.7 & 0.2 & 21.531 & 0.073 & 1 & \\
\hline 20406-1319 & HJ & 611 & 2016.875 & 338.9 & 0.2 & 12.136 & 0.041 & 1 & \\
\hline $20409+1035$ & STF & 2713 & 2016.817 & 62.5 & 0.2 & 5.104 & 0.017 & 1 & \\
\hline $20409+1738$ & $\mathrm{~J}$ & 191 & 2016.817 & 166.5 & 0.2 & 1.321 & 0.004 & 1 & \\
\hline $20411+2133$ & HJ & 922 & 2016.817 & 313.3 & 0.2 & 7.593 & 0.026 & 1 & \\
\hline $20412-1320$ & WSI & 163 & 2016.875 & 46.9 & 0.2 & 10.302 & 0.035 & 1 & B \\
\hline $20414-0835$ & $\mathrm{HDO}$ & 159 & 2016.875 & 236.8 & 0.2 & 8.238 & 0.028 & 1 & \\
\hline $20418-0430$ & HJ & 921 & 2016.817 & 219.6 & 0.2 & 9.412 & 0.032 & 1 & \\
\hline $20431+0253$ & $\mathrm{AG}$ & 262 & 2016.817 & 93.9 & 0.2 & 5.557 & 0.019 & 1 & \\
\hline 20434-1929 & HLD & $40 \mathrm{AB}$ & 2016.730 & 356.5 & 0.2 & 5.228 & 0.018 & 1 & \\
\hline $20435+1657$ & STF & 2720 & 2016.817 & 177.8 & 0.2 & 3.952 & 0.013 & 1 & \\
\hline $20445+2356$ & STF & 2724 & 2016.727 & 149.8 & 0.2 & 2.545 & 0.009 & 1 & \\
\hline $20448+2500$ & BRT & 3361 & 2016.727 & 140.4 & 0.2 & 4.937 & 0.017 & 1 & \\
\hline $20453+6332$ & ES & 134 & 2016.700 & 84.6 & 0.2 & 11.101 & 0.038 & 1 & \\
\hline $20454+3651$ & SEI & 1238 & 2016.683 & 123.7 & 0.2 & 8.130 & 0.028 & 1 & \\
\hline $20456-0853$ & HDO & 160 & 2016.825 & 201.4 & 0.2 & 5.672 & 0.019 & 1 & \\
\hline $20457+3647$ & $\mathrm{AG}$ & 265 & 2016.683 & 207.1 & 0.2 & 6.682 & 0.023 & 1 & \\
\hline $20460+3601$ & SEI & 1242 & 2016.683 & 214.4 & 0.2 & 23.611 & 0.080 & 1 & \\
\hline $20461+2638$ & BRT & 217 & 2016.727 & 80.3 & 0.2 & 4.233 & 0.014 & 1 & \\
\hline
\end{tabular}


Table 4-Continued

\begin{tabular}{|c|c|c|c|c|c|c|c|c|c|}
\hline $\begin{array}{l}\text { WDS Desig. } \\
\alpha, \delta(2000)\end{array}$ & $\begin{array}{l}\text { Dis } \\
\text { Desi }\end{array}$ & $\begin{array}{l}\text { coverer } \\
\text { ignation }\end{array}$ & JY & $\begin{array}{c}\theta \\
(0)\end{array}$ & $\begin{array}{l}\sigma \theta \\
(0)\end{array}$ & $\begin{array}{c}\rho \\
\left({ }^{\prime \prime}\right)\end{array}$ & $\begin{array}{l}\sigma \rho \\
\left({ }^{\prime \prime}\right)\end{array}$ & $\mathrm{n}$ & Note \\
\hline $20464+3747$ & SEI & 1244 & 2016.683 & 149.9 & 0.2 & 22.483 & 0.076 & 1 & \\
\hline $20465-1642$ & SKI & 11 & 2016.730 & 290.3 & 0.2 & 3.961 & 0.013 & 1 & \\
\hline $20466+0344$ & BAL & 2545 & 2016.825 & 281.5 & 0.2 & 11.813 & 0.040 & 1 & \\
\hline $20471+3850$ & HJ & 1575 & 2016.683 & 52.0 & 0.2 & 14.939 & 0.051 & 1 & \\
\hline $20476+3812$ & SEI & 1250 & 2016.683 & 18.9 & 0.2 & 17.339 & 0.059 & 1 & \\
\hline $20484+0426$ & $\mathrm{AG}$ & 267 & 2016.727 & 261.2 & 0.2 & 5.896 & 0.020 & 1 & \\
\hline $20485+3608$ & SEI & 1253 & 2016.683 & 83.4 & 0.2 & 24.075 & 0.082 & 1 & \\
\hline $20489-0214$ & $\mathrm{HO}$ & $142 \mathrm{AB}$ & 2016.825 & 8.9 & 0.2 & 1.008 & 0.003 & 1 & \\
\hline $20489-2037$ & HJ & $2998 \mathrm{AB}$ & 2016.730 & 140.5 & 0.2 & 5.993 & 0.020 & 1 & \\
\hline $20493+2026$ & HJ & 926 & 2016.727 & 189.8 & 0.2 & 5.833 & 0.020 & 1 & \\
\hline $20497+2526$ & $\mathrm{POU}$ & 5028 & 2016.727 & 336.6 & 0.2 & 14.822 & 0.050 & 1 & \\
\hline $20499+1255$ & HJ & 1577 & 2016.727 & 246.0 & 0.2 & 9.382 & 0.032 & 1 & \\
\hline $20505+3635$ & SEI & $1271 \mathrm{AB}$ & 2016.683 & 27.1 & 0.2 & 14.572 & 0.050 & 1 & \\
\hline $20505+3635$ & SEI & $1272 \mathrm{AC}$ & 2016.683 & 184.9 & 0.2 & 19.912 & 0.068 & 1 & \\
\hline $20507-0929$ & HDS & 2970 & 2016.825 & 114.4 & 0.2 & 15.152 & 0.052 & 1 & \\
\hline $20508-1135$ & $\mathrm{~J}$ & $2324 \mathrm{AB}$ & 2016.825 & 192.2 & 0.2 & 27.182 & 0.092 & 1 & \\
\hline $20508-1135$ & $\mathrm{~J}$ & $2324 \mathrm{AC}$ & 2016.825 & 183.8 & 0.2 & 25.795 & 0.088 & 1 & \\
\hline $20508-1135$ & $\mathrm{~J}$ & $2324 \mathrm{BC}$ & 2016.825 & 78.8 & 0.2 & 4.031 & 0.014 & 1 & \\
\hline $20510-0038$ & $\mathrm{~J}$ & 1711 & 2016.825 & 222.7 & 0.2 & 8.442 & 0.029 & 1 & \\
\hline $20510+3717$ & SEI & 1277 & 2016.683 & 32.6 & 0.2 & 8.088 & 0.027 & 1 & \\
\hline $20510+3915$ & SEI & $1279 \mathrm{AB}$ & 2016.683 & 147.4 & 0.2 & 12.935 & 0.044 & 1 & \\
\hline $20510+3915$ & SEI & $1279 \mathrm{BC}$ & 2016.683 & 189.8 & 0.2 & 7.342 & 0.025 & 1 & B \\
\hline $20511+0623$ & STF & 2730 & 2016.727 & 333.6 & 0.2 & 3.409 & 0.012 & 1 & \\
\hline $20517+6701$ & MLB & 302 & 2016.700 & 324.5 & 0.2 & 4.939 & 0.017 & 1 & \\
\hline $20522+2218$ & $\mathrm{~J}$ & 1327 & 2016.875 & 58.6 & 0.2 & 2.738 & 0.009 & 1 & \\
\hline $20537+0336$ & BAL & 2548 & 2016.784 & 257.9 & 0.2 & 7.535 & 0.026 & 1 & \\
\hline $20554+0653$ & BRT & 2188 & 2016.825 & 46.2 & 0.2 & 4.188 & 0.014 & 1 & \\
\hline $20562-0459$ & $\mathrm{BRT}$ & 510 & 2016.784 & 314.8 & 0.2 & 4.796 & 0.016 & 1 & \\
\hline $20567+1300$ & $\mathrm{STF}$ & 2736 & 2016.825 & 219.4 & 0.2 & 5.122 & 0.017 & 1 & \\
\hline $20568+6134$ & STF & 2740 & 2016.853 & 331.3 & 0.2 & 4.110 & 0.014 & 1 & \\
\hline $20575+0036$ & HWE & $101 \mathrm{AB}$ & 2016.784 & 139.6 & 0.2 & 40.658 & 0.138 & 1 & \\
\hline $20575+0036$ & HWE & $101 \mathrm{AC}$ & 2016.784 & 313.3 & 0.2 & 11.714 & 0.040 & 1 & \\
\hline
\end{tabular}


Table 4-Continued

\begin{tabular}{|c|c|c|c|c|c|c|c|c|c|}
\hline $\begin{array}{l}\text { WDS Desig. } \\
\alpha, \delta(2000)\end{array}$ & & $\begin{array}{l}\text { iscoverer } \\
\text { signation }\end{array}$ & JY & $\begin{array}{c}\theta \\
(0)\end{array}$ & $\begin{array}{l}\sigma \theta \\
(0)\end{array}$ & $\begin{array}{c}\rho \\
\left({ }^{\prime \prime}\right)\end{array}$ & $\begin{array}{l}\sigma \rho \\
\left({ }^{\prime \prime}\right)\end{array}$ & $\mathrm{n}$ & Note \\
\hline $20581-1237$ & $\mathrm{HU}$ & 82 & 2016.648 & 2.7 & 0.2 & 2.737 & 0.009 & 1 & \\
\hline $20591+0418$ & STF & $2737 \mathrm{AB}, \mathrm{C}$ & 2016.784 & 66.7 & 0.2 & 10.624 & 0.036 & 1 & \\
\hline $20597+2418$ & BRT & 220 & 2016.825 & 131.8 & 0.2 & 4.922 & 0.017 & 1 & \\
\hline $20598+2004$ & STF & 2739 & 2016.825 & 253.0 & 0.2 & 3.304 & 0.011 & 1 & \\
\hline $20598+6152$ & $\mathrm{BU}$ & 472 & 2016.700 & 14.2 & 0.2 & 0.770 & 0.003 & 1 & \\
\hline $21006+5432$ & HJ & $1606 \mathrm{AB}$ & 2016.853 & 184.9 & 0.2 & 18.740 & 0.064 & 1 & \\
\hline $21009+5931$ & ES & 2703 & 2016.853 & 231.0 & 0.2 & 10.165 & 0.035 & 1 & \\
\hline $21015+6643$ & $\mathrm{HU}$ & 959 & 2016.700 & 162.1 & 0.2 & 1.299 & 0.004 & 1 & \\
\hline $21031+5519$ & ES & 1001 & 2016.784 & 34.2 & 0.2 & 4.068 & 0.014 & 1 & \\
\hline $21037+3842$ & SEI & 1384 & 2016.664 & 95.8 & 0.2 & 26.368 & 0.090 & 1 & \\
\hline $21039+4138$ & STT & $\mathrm{A} 214 \mathrm{AB}$ & 2016.703 & 184.7 & 0.2 & 57.725 & 0.196 & 1 & \\
\hline $21043+4806$ & ARN & 51 & 2016.703 & 340.4 & 0.2 & 47.933 & 0.163 & 1 & \\
\hline $21044+4057$ & $\mathrm{ROE}$ & 44 & 2016.664 & 43.0 & 0.2 & 7.025 & 0.024 & 1 & \\
\hline $21044+4626$ & DAL & 49 & 2016.703 & 147.6 & 0.2 & 12.135 & 0.041 & 1 & \\
\hline $21045+2730$ & $\mathrm{~J}$ & 1180 & 2016.760 & 276.0 & 0.2 & 4.040 & 0.014 & 1 & \\
\hline $21045+7046$ & STF & 2771 & 2016.656 & 34.0 & 0.2 & 2.731 & 0.009 & 1 & \\
\hline $21046+2053$ & $\mathrm{AG}$ & 269 & 2016.760 & 173.1 & 0.2 & 8.437 & 0.029 & 1 & \\
\hline $21046+3345$ & CXT & 1 & 2016.760 & 178.6 & 0.2 & 7.672 & 0.026 & 1 & \\
\hline $21047+3908$ & SEI & 1393 & 2016.664 & 131.7 & 0.2 & 26.204 & 0.089 & 1 & \\
\hline $21052-1004$ & $\mathrm{AOT}$ & 104 & 2016.648 & 174.1 & 0.2 & 7.420 & 0.025 & 1 & \\
\hline $21055+6210$ & STF & $2764 \mathrm{~A}, \mathrm{BC}$ & 2016.700 & 298.0 & 0.2 & 7.061 & 0.024 & 1 & \\
\hline $21057+3215$ & $\mathrm{KU}$ & 130 & 2016.760 & 57.8 & 0.2 & 19.446 & 0.066 & 1 & \\
\hline $21058+3929$ & A & $2691 \mathrm{AC}$ & 2016.664 & 314.1 & 0.2 & 27.441 & 0.093 & 1 & \\
\hline $21059+3232$ & GYL & 48 & 2016.760 & 180.7 & 0.2 & 32.253 & 0.110 & 1 & \\
\hline $21061+4448$ & $\mathrm{ROE}$ & $45 \mathrm{BF}$ & 2016.703 & 313.0 & 0.2 & 10.819 & 0.037 & 1 & \\
\hline $21061+4448$ & $\mathrm{ROE}$ & $45 \mathrm{DE}$ & 2016.703 & 6.5 & 0.2 & 27.540 & 0.094 & 1 & \\
\hline $21063+5308$ & ES & 2705 & 2016.784 & 18.5 & 0.2 & 9.006 & 0.031 & 1 & \\
\hline $21065+4823$ & $\mathrm{BU}$ & $836 \mathrm{AB}$ & 2016.703 & 174.1 & 0.2 & 0.775 & 0.003 & 1 & \\
\hline $21065+4823$ & $\mathrm{BU}$ & $836 \mathrm{AB}, \mathrm{C}$ & 2016.703 & 218.7 & 0.2 & 27.495 & 0.093 & 1 & \\
\hline $21066-0534$ & BRT & 557 & 2016.648 & 281.1 & 0.2 & 4.271 & 0.015 & 1 & \\
\hline $21067+4631$ & SMA & $125 \mathrm{AB}$ & 2016.703 & 87.8 & 0.2 & 22.228 & 0.076 & 1 & \\
\hline $21067+4631$ & SMA & $125 \mathrm{AC}$ & 2016.703 & 198.6 & 0.2 & 14.966 & 0.051 & 1 & \\
\hline
\end{tabular}


Table 4-Continued

\begin{tabular}{|c|c|c|c|c|c|c|c|c|c|}
\hline $\begin{array}{l}\text { WDS Desig. } \\
\alpha, \delta(2000)\end{array}$ & $\begin{array}{l}\text { Dis } \\
\text { Desi }\end{array}$ & $\begin{array}{l}\text { coverer } \\
\text { ignation }\end{array}$ & JY & $\begin{array}{c}\theta \\
(0)\end{array}$ & $\begin{array}{l}\sigma \theta \\
(\mathrm{o})\end{array}$ & $\begin{array}{c}\rho \\
\left({ }^{\prime \prime}\right)\end{array}$ & $\begin{array}{l}\sigma \rho \\
\left({ }^{\prime \prime}\right)\end{array}$ & $\mathrm{n}$ & Note \\
\hline $21070-0435$ & BRT & 512 & 2016.648 & 63.9 & 0.2 & 5.229 & 0.018 & 1 & \\
\hline $21070+5900$ & $\mathrm{STF}$ & 2766 & 2016.784 & 248.6 & 0.2 & 4.537 & 0.015 & 1 & \\
\hline $21074+2429$ & $\mathrm{STF}$ & 2761 & 2016.760 & 111.6 & 0.2 & 5.622 & 0.019 & 1 & \\
\hline $21075-0010$ & $\mathrm{STF}$ & 2755 & 2016.648 & 79.5 & 0.2 & 25.390 & 0.086 & 1 & \\
\hline $21082+4055$ & $\mathrm{AG}$ & 414 & 2016.664 & 107.5 & 0.2 & 5.174 & 0.018 & 1 & \\
\hline $21086+3942$ & ALI & 1154 & 2016.664 & 251.7 & 0.2 & 12.530 & 0.043 & 1 & \\
\hline $21092+2820$ & MLB & 537 & 2016.760 & 99.3 & 0.2 & 7.001 & 0.024 & 1 & \\
\hline $21096+5502$ & HJ & 1621 & 2016.784 & 148.8 & 0.2 & 19.410 & 0.066 & 1 & \\
\hline $21098+2915$ & $\mathrm{DU}$ & 3 & 2016.760 & 15.6 & 0.2 & 6.123 & 0.021 & 1 & \\
\hline $21099+4422$ & $\mathrm{STF}$ & 2772 & 2016.703 & 224.9 & 0.2 & 12.437 & 0.042 & 1 & \\
\hline $21100+4326$ & $\mathrm{ES}$ & 1453 & 2016.703 & 68.1 & 0.2 & 5.368 & 0.018 & 1 & \\
\hline $21101+0118$ & $\mathrm{HDO}$ & 318 & 2016.648 & 314.2 & 0.2 & 1.081 & 0.004 & 1 & \\
\hline $21103-1304$ & $\mathrm{CBL}$ & 181 & 2016.648 & 246.8 & 0.2 & 17.145 & 0.058 & 1 & \\
\hline $21103+4359$ & $\mathrm{STF}$ & $2773 \mathrm{AB}$ & 2016.703 & 113.3 & 0.2 & 3.285 & 0.011 & 1 & \\
\hline $21105+1958$ & $\mathrm{STF}$ & 2767 & 2016.648 & 28.5 & 0.2 & 2.488 & 0.008 & 1 & \\
\hline $21105+2227$ & $\mathrm{STF}$ & $2769 \mathrm{AB}$ & 2016.760 & 299.5 & 0.2 & 18.276 & 0.062 & 1 & \\
\hline $21110+0933$ & $\mathrm{STF}$ & $2765 \mathrm{AB}$ & 2016.648 & 79.3 & 0.2 & 2.780 & 0.009 & 1 & \\
\hline $21112+5620$ & $\mathrm{DOO}$ & 16 & 2016.784 & 29.2 & 0.2 & 1.130 & 0.004 & 1 & \\
\hline $21113+3419$ & $\mathrm{ES}$ & 2314 & 2016.760 & 189.1 & 0.2 & 4.476 & 0.015 & 1 & \\
\hline $21115+4115$ & $\mathrm{STT}$ & $431 \mathrm{AB}$ & 2016.664 & 126.0 & 0.2 & 2.636 & 0.009 & 1 & \\
\hline $21116+1251$ & $\mathrm{ROE}$ & 102 & 2016.648 & 128.2 & 0.2 & 6.540 & 0.022 & 1 & \\
\hline $21118+5959$ & $\mathrm{STF}$ & $2780 \mathrm{AB}$ & 2016.784 & 212.9 & 0.2 & 1.016 & 0.003 & 1 & \\
\hline $21119+4838$ & HJ & 1624 & 2016.703 & 189.8 & 0.2 & 6.073 & 0.021 & 1 & \\
\hline $21123+3453$ & SLE & $370 \mathrm{AC}$ & 2016.760 & 199.8 & 0.2 & 16.237 & 0.055 & 1 & \\
\hline $21125+3217$ & SEI & 1443 & 2016.760 & 139.9 & 0.2 & 25.586 & 0.087 & 1 & \\
\hline $21129+4820$ & HJ & 1625 & 2016.839 & 71.4 & 0.2 & 11.831 & 0.040 & 1 & \\
\hline $21131+3249$ & SEI & 1446 & 2016.659 & 155.4 & 0.2 & 8.681 & 0.030 & 1 & \\
\hline $21133+2041$ & $\mathrm{BRT}$ & 2493 & 2016.659 & 316.8 & 0.2 & 4.342 & 0.015 & 1 & \\
\hline $21135-0905$ & $\mathrm{BRT}$ & 515 & 2016.727 & 68.0 & 0.2 & 4.119 & 0.014 & 1 & \\
\hline $21144+5015$ & $\mathrm{SKF}$ & 393 & 2016.661 & 206.1 & 0.2 & 44.564 & 0.152 & 1 & \\
\hline $21147+5702$ & HJ & 1630 & 2016.661 & 133.9 & 0.2 & 17.439 & 0.059 & 1 & \\
\hline $21152+5351$ & BVD & 134 & 2016.661 & 57.5 & 0.2 & 22.856 & 0.078 & 1 & \\
\hline
\end{tabular}


Table 4-Continued

\begin{tabular}{|c|c|c|c|c|c|c|c|c|c|}
\hline $\begin{array}{l}\text { WDS Desig. } \\
\alpha, \delta(2000)\end{array}$ & $\begin{array}{r}\text { Dis } \\
\text { Des }\end{array}$ & $\begin{array}{l}\text { coverer } \\
\text { ignation }\end{array}$ & JY & $\begin{array}{c}\theta \\
(\circ)\end{array}$ & $\begin{array}{l}\sigma \theta \\
(\circ)\end{array}$ & $\begin{array}{c}\rho \\
\left({ }^{\prime \prime}\right)\end{array}$ & $\begin{array}{l}\sigma \rho \\
\left({ }^{\prime \prime}\right)\end{array}$ & $\mathrm{n}$ & Note \\
\hline $21154-1021$ & STF & $2776 \mathrm{AB}$ & 2016.727 & 52.9 & 0.2 & 83.486 & 0.284 & 1 & \\
\hline $21154-1021$ & $\mathrm{STF}$ & $2776 \mathrm{BC}$ & 2016.727 & 338.2 & 0.2 & 5.917 & 0.020 & 1 & \\
\hline $21157+3235$ & HJ & 1628 & 2016.659 & 251.8 & 0.2 & 15.288 & 0.052 & 1 & \\
\hline $21158+6721$ & $\mathrm{STF}$ & 2788 & 2016.659 & 353.1 & 0.2 & 8.073 & 0.027 & 1 & \\
\hline $21167-0739$ & $\mathrm{STF}$ & 2781 & 2016.727 & 172.0 & 0.2 & 2.720 & 0.009 & 1 & \\
\hline $21175+7658$ & LDS & 1948 & 2016.659 & 305.4 & 0.2 & 8.677 & 0.030 & 1 & \\
\hline $21176+8231$ & $\mathrm{STF}$ & 2807 & 2016.656 & 310.6 & 0.2 & 1.834 & 0.006 & 1 & \\
\hline $21177+1636$ & $\mathrm{~J}$ & $850 \mathrm{AC}$ & 2016.659 & 331.2 & 0.2 & 12.438 & 0.042 & 1 & \\
\hline $21185+8021$ & $\mathrm{STF}$ & 2801 & 2016.659 & 271.7 & 0.2 & 2.128 & 0.007 & 1 & \\
\hline $21189+5938$ & MLB & 1092 & 2016.661 & 106.2 & 0.2 & 10.477 & 0.036 & 1 & \\
\hline $21194+5219$ & ES & $98 \mathrm{AB}$ & 2016.661 & 309.1 & 0.2 & 25.936 & 0.088 & 1 & \\
\hline $21194+5219$ & ES & $98 \mathrm{AC}$ & 2016.661 & 87.6 & 0.2 & 29.847 & 0.101 & 1 & \\
\hline $21197+0931$ & $\mathrm{STF}$ & 2786 & 2016.727 & 188.7 & 0.2 & 2.817 & 0.010 & 1 & \\
\hline $21197+5303$ & $\mathrm{~S}$ & 786 & 2016.661 & 299.4 & 0.2 & 48.002 & 0.163 & 1 & \\
\hline $21200+5436$ & SKF & $1978 \mathrm{AB}$ & 2016.661 & 298.7 & 0.2 & 31.758 & 0.108 & 1 & \\
\hline $21203+4921$ & $\mathrm{BU}$ & $839 \mathrm{AB}$ & 2016.839 & 207.9 & 0.2 & 14.520 & 0.049 & 1 & \\
\hline $21203+4921$ & $\mathrm{BU}$ & $839 \mathrm{AC}$ & 2016.803 & 200.7 & 0.1 & 22.495 & 0.047 & 2 & \\
\hline $21203+4921$ & $\mathrm{BU}$ & $839 \mathrm{CB}$ & 2016.697 & 7.9 & 0.2 & 8.129 & 0.002 & 2 & \\
\hline $21206+1537$ & $\mathrm{AG}$ & 416 & 2016.659 & 351.3 & 0.2 & 23.825 & 0.081 & 1 & \\
\hline $21212+1841$ & $\mathrm{~J}$ & 160 & 2016.659 & 290.2 & 0.2 & 3.237 & 0.011 & 1 & \\
\hline $21213+6042$ & $\mathrm{STF}$ & 2795 & 2016.661 & 301.3 & 0.2 & 1.778 & 0.006 & 1 & \\
\hline $21214+0253$ & STT & 435 & 2016.653 & 238.5 & 0.2 & 0.691 & 0.002 & 1 & \\
\hline $21214-1219$ & HJ & 280 & 2016.653 & 212.1 & 0.2 & 47.794 & 0.163 & 1 & \\
\hline $21225+5504$ & HJ & 1642 & 2016.814 & 210.5 & 0.2 & 35.949 & 0.122 & 1 & \\
\hline $21230+2858$ & $\mathrm{STF}$ & $2792 \mathrm{AB}$ & 2016.730 & 330.5 & 0.2 & 7.231 & 0.025 & 1 & \\
\hline $21230+3202$ & $\mathrm{HO}$ & 157 & 2016.779 & 26.4 & 0.2 & 3.606 & 0.012 & 1 & \\
\hline $21234+5923$ & $\mathrm{ES}$ & 2709 & 2016.785 & 275.4 & 0.2 & 12.191 & 0.041 & 1 & \\
\hline $21236+6456$ & $\mathrm{STF}$ & 2798 & 2016.700 & 143.4 & 0.2 & 6.387 & 0.022 & 1 & \\
\hline $21237+0422$ & STF & 2791 & 2016.653 & 103.7 & 0.2 & 2.864 & 0.010 & 1 & \\
\hline $21240+2236$ & $\mathrm{KU}$ & 60 & 2016.730 & 228.1 & 0.2 & 7.175 & 0.024 & 1 & \\
\hline $21249-1435$ & FOX & 260 & 2016.727 & 30.3 & 0.2 & 11.030 & 0.038 & 1 & \\
\hline $21252+3129$ & $\mathrm{~A}$ & 1220 & 2016.730 & 159.3 & 0.2 & 1.698 & 0.006 & 1 & \\
\hline
\end{tabular}


Table 4-Continued

\begin{tabular}{|c|c|c|c|c|c|c|c|c|c|}
\hline \multirow{2}{*}{$\begin{array}{c}\text { WDS Desig. } \\
\alpha, \delta(2000) \\
21257+7233\end{array}$} & \multicolumn{2}{|c|}{$\begin{array}{c}\text { Discoverer } \\
\text { Designation }\end{array}$} & \multirow{2}{*}{$\begin{array}{c}\text { JY } \\
2016.700\end{array}$} & \multirow{2}{*}{$\begin{array}{c}\theta \\
(0) \\
127.5\end{array}$} & \multirow{2}{*}{$\begin{array}{l}\sigma \theta \\
(0) \\
0.2\end{array}$} & \multirow{2}{*}{$\begin{array}{c}\rho \\
\left({ }^{\prime \prime}\right) \\
23.226\end{array}$} & \multirow{2}{*}{$\begin{array}{c}\begin{array}{c}\sigma \rho \\
\left({ }^{\prime \prime}\right)\end{array} \\
0.079\end{array}$} & \multirow{2}{*}{$\begin{array}{l}\mathrm{n} \\
\\
1\end{array}$} & \multirow{2}{*}{$\begin{array}{l}\text { Note } \\
\end{array}$} \\
\hline & HJ & $3035 \mathrm{AB}$ & & & & & & & \\
\hline $21257+7233$ & HJ & $3035 \mathrm{AC}$ & 2016.700 & 202.8 & 0.2 & 24.180 & 0.082 & 1 & \\
\hline $21267+3413$ & $\mathrm{ES}$ & 2261 & 2016.779 & 155.8 & 0.2 & 4.124 & 0.014 & 1 & \\
\hline $21268+0642$ & HJ & 3028 & 2016.653 & 242.3 & 0.2 & 20.961 & 0.071 & 1 & \\
\hline $21279-1326$ & SCJ & 28 & 2016.653 & 134.8 & 0.2 & 3.047 & 0.010 & 1 & \\
\hline 21282-1906 & HJ & 3029 & 2016.727 & 352.6 & 0.2 & 19.001 & 0.065 & 1 & \\
\hline $21287+6137$ & HJ & 1654 & 2016.700 & 35.6 & 0.2 & 4.887 & 0.017 & 1 & \\
\hline $21301+1514$ & $\mathrm{~J}$ & 198 & 2016.653 & 38.5 & 0.2 & 4.171 & 0.014 & 1 & \\
\hline $21304+3504$ & HLD & 45 & 2016.779 & 198.0 & 0.2 & 1.295 & 0.004 & 1 & \\
\hline $21306+5954$ & HJ & 3038 & 2016.785 & 113.1 & 0.2 & 17.166 & 0.058 & 1 & \\
\hline $21318+3349$ & STF & 2802 & 2016.779 & 9.6 & 0.2 & 3.866 & 0.013 & 1 & \\
\hline $21326+7134$ & HJ & 3044 & 2016.700 & 75.4 & 0.2 & 7.624 & 0.026 & 1 & \\
\hline $21327+0751$ & HJ & 937 & 2016.653 & 341.9 & 0.2 & 10.611 & 0.036 & 1 & \\
\hline $21336+3325$ & TOB & $222 \mathrm{AB}$ & 2016.779 & 119.0 & 0.2 & 21.019 & 0.071 & 1 & \\
\hline $21336+3325$ & TOB & $222 \mathrm{AC}$ & 2016.779 & 335.2 & 0.2 & 22.254 & 0.076 & 1 & \\
\hline $21336+3325$ & TOB & $222 \mathrm{AD}$ & 2016.779 & 67.9 & 0.2 & 26.449 & 0.090 & 1 & \\
\hline $21344+1552$ & $\mathrm{~J}$ & 285 & 2016.653 & 72.2 & 0.2 & 2.479 & 0.008 & 1 & \\
\hline $21348+5941$ & STF & 2812 & 2016.785 & 133.1 & 0.2 & 2.409 & 0.008 & 1 & \\
\hline $21353+3020$ & MLB & 718 & 2016.730 & 307.8 & 0.2 & 8.101 & 0.028 & 1 & \\
\hline $21355-1846$ & I & $380 \mathrm{AB}$ & 2016.727 & 354.8 & 0.2 & 1.158 & 0.004 & 1 & \\
\hline $21360+5728$ & STF & 2813 & 2016.661 & 272.7 & 0.2 & 10.234 & 0.035 & 1 & \\
\hline $21361-1023$ & HJ & 5518 & 2016.730 & 159.2 & 0.2 & 53.569 & 0.182 & 1 & \\
\hline $21361+2716$ & MLB & 620 & 2016.880 & 243.1 & 0.2 & 7.450 & 0.025 & 1 & \\
\hline $21363+7247$ & HJ & 3051 & 2016.700 & 183.4 & 0.2 & 13.526 & 0.046 & 1 & \\
\hline $21368+4332$ & HJ & 1666 & 2016.891 & 239.1 & 0.2 & 8.087 & 0.027 & 1 & \\
\hline $21373+5050$ & HJ & 1671 & 2016.760 & 326.2 & 0.2 & 9.658 & 0.033 & 1 & \\
\hline $21373+5244$ & $\mathrm{~A}$ & $1700 \mathrm{AB}$ & 2016.662 & 296.5 & 0.2 & 4.999 & 0.017 & 1 & \\
\hline $21376+5546$ & STT & $\mathrm{A} 220 \mathrm{AC}$ & 2016.662 & 10.2 & 0.2 & 41.428 & 0.141 & 1 & \\
\hline $21376+5546$ & STT & A220 AD & 2016.661 & 285.6 & 0.2 & 40.947 & 0.139 & 1 & \\
\hline $21377+0637$ & STFA & $56 \mathrm{AB}$ & 2016.730 & 348.3 & 0.2 & 39.307 & 0.134 & 1 & \\
\hline $21377+5734$ & STF & $2815 \mathrm{AB}, \mathrm{C}$ & 2016.661 & 81.7 & 0.2 & 7.501 & 0.026 & 1 & \\
\hline $21380-1913$ & LDS & 751 & 2016.656 & 356.1 & 0.2 & 18.924 & 0.064 & 1 & \\
\hline
\end{tabular}


Table 4-Continued

\begin{tabular}{|c|c|c|c|c|c|c|c|c|c|}
\hline $\begin{array}{l}\text { WDS Desig. } \\
\alpha, \delta(2000)\end{array}$ & & $\begin{array}{l}\text { iscoverer } \\
\text { signation }\end{array}$ & JY & $\begin{array}{c}\theta \\
(0)\end{array}$ & $\begin{array}{l}\sigma \theta \\
(0)\end{array}$ & $\begin{array}{c}\rho \\
\left({ }^{\prime \prime}\right)\end{array}$ & $\begin{array}{l}\sigma \rho \\
\left({ }^{\prime \prime}\right)\end{array}$ & $\mathrm{n}$ & Note \\
\hline $21380+4829$ & ES & 102 & 2016.853 & 36.3 & 0.2 & 11.418 & 0.039 & 1 & \\
\hline $21385+3942$ & MLB & 786 & 2016.891 & 210.1 & 0.2 & 5.277 & 0.018 & 1 & \\
\hline $21389+3623$ & STF & 2814 & 2016.880 & 160.3 & 0.2 & 7.939 & 0.027 & 1 & \\
\hline $21390+4940$ & HJ & 1674 & 2016.853 & 328.3 & 0.2 & 11.086 & 0.038 & 1 & \\
\hline $21390+5729$ & GUI & $36 \mathrm{CD}$ & 2016.661 & 324.6 & 0.2 & 30.312 & 0.103 & 1 & \\
\hline $21390+5729$ & STF & $2816 \mathrm{AC}$ & 2016.661 & 120.2 & 0.2 & 11.913 & 0.041 & 1 & \\
\hline $21390+5729$ & STF & $2816 \mathrm{AD}$ & 2016.661 & 338.8 & 0.2 & 19.996 & 0.068 & 1 & \\
\hline $21391+4421$ & HJ & 1673 & 2016.891 & 81.0 & 0.2 & 5.360 & 0.018 & 1 & \\
\hline $21393+2043$ & STT & 445 & 2016.826 & 120.7 & 0.2 & 1.028 & 0.003 & 1 & \\
\hline $21394+5255$ & SKF & 349 & 2016.662 & 266.3 & 0.2 & 14.005 & 0.048 & 1 & \\
\hline $21397+5815$ & MLB & $90 \mathrm{AB}$ & 2016.760 & 36.6 & 0.2 & 8.173 & 0.028 & 1 & \\
\hline $21397+5815$ & MLB & $90 \mathrm{AC}$ & 2016.760 & 136.4 & 0.2 & 29.744 & 0.101 & 1 & \\
\hline $21399+4908$ & ES & $825 \mathrm{AC}$ & 2016.853 & 285.0 & 0.2 & 57.715 & 0.196 & 1 & \\
\hline $21401+4824$ & HJ & 1681 & 2016.853 & 107.9 & 0.2 & 7.530 & 0.026 & 1 & \\
\hline $21402-1826$ & $\mathrm{COO}$ & 243 & 2016.730 & 65.3 & 0.2 & 4.919 & 0.017 & 1 & \\
\hline $21403+0344$ & STT & 446 & 2016.730 & 173.6 & 0.2 & 6.798 & 0.023 & 1 & \\
\hline $21420+0408$ & $\mathrm{~J}$ & 1790 & 2016.826 & 96.1 & 0.2 & 2.288 & 0.008 & 1 & \\
\hline $21422+4434$ & $\mathrm{ES}$ & $1341 \mathrm{AB}$ & 2016.891 & 280.8 & 0.2 & 5.890 & 0.020 & 1 & \\
\hline $21422+4434$ & ES & $1341 \mathrm{AC}$ & 2016.891 & 327.6 & 0.2 & 33.489 & 0.114 & 1 & \\
\hline $21429+3904$ & GRV & 496 & 2016.883 & 72.9 & 0.2 & 29.894 & 0.102 & 1 & \\
\hline $21431+2157$ & BRT & 2499 & 2016.826 & 206.7 & 0.2 & 4.844 & 0.016 & 1 & \\
\hline $21433+2046$ & $\mathrm{~J}$ & 852 & 2016.826 & 93.0 & 0.2 & 4.217 & 0.014 & 1 & \\
\hline $21434+4417$ & HJ & 1685 & 2016.853 & 240.0 & 0.2 & 18.613 & 0.063 & 1 & \\
\hline $21436+0700$ & HJ & 3053 & 2016.730 & 191.1 & 0.2 & 24.278 & 0.083 & 1 & \\
\hline $21436+5827$ & LDS & 4905 & 2016.760 & 227.3 & 0.2 & 41.401 & 0.141 & 1 & \\
\hline $21439+5034$ & $\mathrm{HU}$ & 693 & 2016.760 & 233.9 & 0.2 & 1.127 & 0.004 & 1 & \\
\hline $21439+6336$ & STF & $2827 \mathrm{~A}, \mathrm{BC}$ & 2016.700 & 199.0 & 0.2 & 5.871 & 0.020 & 1 & \\
\hline $21444+0008$ & CHE & 316 & 2016.730 & 297.9 & 0.2 & 38.413 & 0.131 & 1 & \\
\hline $21448+4931$ & $\mathrm{ARG}$ & $43 \mathrm{AB}$ & 2016.760 & 19.9 & 0.2 & 29.283 & 0.100 & 1 & \\
\hline $21449+3322$ & $\mathrm{ES}$ & $2385 \mathrm{AC}$ & 2016.880 & 358.7 & 0.2 & 26.685 & 0.091 & 1 & \\
\hline $21451+2512$ & $\mathrm{POU}$ & 5482 & 2016.825 & 94.5 & 0.2 & 17.368 & 0.059 & 1 & \\
\hline $21452+5431$ & HDS & 3098 & 2016.662 & 61.6 & 0.2 & 19.940 & 0.068 & 1 & \\
\hline
\end{tabular}


Table 4-Continued

\begin{tabular}{|c|c|c|c|c|c|c|c|c|c|}
\hline $\begin{array}{l}\text { WDS Desig. } \\
\alpha, \delta(2000)\end{array}$ & \multicolumn{2}{|c|}{$\begin{array}{l}\text { Discoverer } \\
\text { Designation }\end{array}$} & JY & $\begin{array}{c}\theta \\
(0)\end{array}$ & $\begin{array}{l}\sigma \theta \\
(0)\end{array}$ & $\begin{array}{c}\rho \\
\left({ }^{\prime \prime}\right)\end{array}$ & $\begin{array}{l}\sigma \rho \\
\left({ }^{\prime \prime}\right)\end{array}$ & $\mathrm{n}$ & Note \\
\hline $21454+4258$ & $\mathrm{ES}$ & 1459 & 2016.883 & 283.1 & 0.2 & 2.333 & 0.008 & 1 & \\
\hline $21455+2156$ & $\mathrm{AG}$ & 276 & 2016.825 & 2.0 & 0.2 & 1.970 & 0.007 & 1 & \\
\hline $21456+0251$ & BAL & 2056 & 2016.730 & 269.2 & 0.2 & 7.922 & 0.027 & 1 & \\
\hline $21458+1545$ & STT & A 224 & 2016.826 & 359.7 & 0.2 & 59.880 & 0.204 & 1 & \\
\hline $21461+2111$ & $\mathrm{AG}$ & 277 & 2016.825 & 60.8 & 0.2 & 2.681 & 0.009 & 1 & \\
\hline $21462+5748$ & ES & $36 \mathrm{BC}$ & 2016.661 & 138.6 & 0.2 & 7.109 & 0.024 & 1 & \\
\hline $21462+5748$ & HJ & $1694 \mathrm{AB}$ & 2016.661 & 13.6 & 0.2 & 19.852 & 0.067 & 1 & \\
\hline $21467+5523$ & ES & 1012 & 2016.661 & 6.8 & 0.2 & 4.369 & 0.015 & 1 & \\
\hline $21468+1030$ & $\mathrm{~J}$ & 201 & 2016.826 & 224.9 & 0.2 & 2.748 & 0.009 & 1 & \\
\hline $21473+4644$ & SRW & $11 \mathrm{AB}$ & 2016.853 & 28.2 & 0.2 & 28.920 & 0.098 & 1 & \\
\hline $21473+4644$ & SRW & $11 \mathrm{AC}$ & 2016.853 & 341.4 & 0.2 & 26.251 & 0.089 & 1 & $\mathrm{C}$ \\
\hline $21474-1307$ & STF & $2826 \mathrm{AC}$ & 2016.730 & 81.5 & 0.2 & 3.987 & 0.014 & 1 & \\
\hline $21480-2022$ & ARA & 1229 & 2016.656 & 64.1 & 0.2 & 13.373 & 0.045 & 1 & \\
\hline $21480+6920$ & STF & 2835 & 2016.700 & 271.5 & 0.2 & 1.879 & 0.006 & 1 & \\
\hline $21485+6137$ & $\mathrm{D}$ & $33 \mathrm{AB}$ & 2016.700 & 131.6 & 0.2 & 3.509 & 0.012 & 1 & \\
\hline $21485+6137$ & MLB & $176 \mathrm{AC}$ & 2016.700 & 277.9 & 0.2 & 10.559 & 0.036 & 1 & \\
\hline $21486+5136$ & HLD & 48 & 2016.760 & 23.0 & 0.2 & 4.630 & 0.016 & 1 & \\
\hline $21490+4316$ & BRT & 1152 & 2016.891 & 231.8 & 0.2 & 6.664 & 0.023 & 1 & \\
\hline $21492+5031$ & STF & $2832 \mathrm{AB}$ & 2016.760 & 212.4 & 0.2 & 13.132 & 0.045 & 1 & \\
\hline $21492+5031$ & STF & $2832 \mathrm{AC}$ & 2016.760 & 320.1 & 0.2 & 46.137 & 0.157 & 1 & \\
\hline $21492+5031$ & STF & $2832 \mathrm{BC}$ & 2016.760 & 334.1 & 0.2 & 51.661 & 0.176 & 1 & \\
\hline $21493+3937$ & SEI & 1536 & 2016.664 & 147.2 & 0.2 & 28.122 & 0.096 & 1 & \\
\hline $21494+5830$ & HJ & 3063 & 2016.703 & 65.1 & 0.2 & 11.474 & 0.039 & 1 & \\
\hline $21495+5349$ & HJ & $3062 \mathrm{AB}$ & 2016.683 & 124.1 & 0.2 & 21.423 & 0.073 & 1 & \\
\hline $21495+5349$ & HJ & $3066 \mathrm{AC}$ & 2016.683 & 202.3 & 0.2 & 30.825 & 0.105 & 1 & \\
\hline $21495+5349$ & HJ & $3066 \mathrm{AD}$ & 2016.683 & 181.5 & 0.2 & 36.306 & 0.123 & 1 & \\
\hline $21495+5834$ & STI & 2588 & 2016.703 & 112.8 & 0.2 & 4.886 & 0.017 & 1 & \\
\hline $21509+3918$ & MLB & 788 & 2016.757 & 52.3 & 0.2 & 4.907 & 0.017 & 1 & \\
\hline $21510+5621$ & $\mathrm{KR}$ & $55 \mathrm{AB}$ & 2016.703 & 355.3 & 0.2 & 4.683 & 0.016 & 1 & \\
\hline $21510+5621$ & $\mathrm{KR}$ & $55 \mathrm{AC}$ & 2016.703 & 20.6 & 0.2 & 16.052 & 0.055 & 1 & \\
\hline $21510+6137$ & STT & 451 & 2016.700 & 218.5 & 0.2 & 4.170 & 0.014 & 1 & \\
\hline $21514+3730$ & SEI & 1540 & 2016.757 & 207.8 & 0.2 & 23.598 & 0.080 & 1 & \\
\hline
\end{tabular}


Table 4-Continued

\begin{tabular}{|c|c|c|c|c|c|c|c|c|c|}
\hline $\begin{array}{l}\text { WDS Desig. } \\
\alpha, \delta(2000)\end{array}$ & & $\begin{array}{l}\text { iscoverer } \\
\text { signation }\end{array}$ & JY & $\begin{array}{c}\theta \\
(0)\end{array}$ & $\begin{array}{l}\sigma \theta \\
(0)\end{array}$ & $\begin{array}{c}\rho \\
(")\end{array}$ & $\begin{array}{l}\sigma \rho \\
\left({ }^{\prime \prime}\right)\end{array}$ & $\mathrm{n}$ & Note \\
\hline $21515+4706$ & HJ & 1701 & 2016.683 & 210.3 & 0.2 & 14.540 & 0.049 & 1 & \\
\hline $21516+6545$ & STF & $2843 \mathrm{AB}$ & 2016.703 & 151.0 & 0.2 & 1.332 & 0.005 & 1 & \\
\hline $21517+2134$ & BRT & 2501 & 2016.757 & 324.2 & 0.2 & 5.162 & 0.018 & 1 & \\
\hline $21521+6206$ & $\mathrm{KR}$ & 56 & 2016.700 & 299.7 & 0.2 & 4.723 & 0.016 & 1 & \\
\hline $21523+6306$ & STF & $2845 \mathrm{AB}$ & 2016.700 & 173.2 & 0.2 & 1.985 & 0.007 & 1 & \\
\hline $21530+4121$ & GRV & 516 & 2016.664 & 151.6 & 0.2 & 14.346 & 0.049 & 1 & \\
\hline $21534+4921$ & ES & $524 \mathrm{AB}$ & 2016.683 & 188.5 & 0.2 & 20.891 & 0.071 & 1 & \\
\hline $21534+4921$ & ES & $524 \mathrm{BC}$ & 2016.683 & 3.6 & 0.2 & 4.722 & 0.016 & 1 & \\
\hline $21537-1645$ & HJ & $615 \mathrm{AB}$ & 2016.656 & 66.8 & 0.2 & 12.459 & 0.042 & 1 & \\
\hline $21538+2856$ & MLB & 495 & 2016.760 & 134.4 & 0.2 & 4.798 & 0.016 & 1 & \\
\hline $21541+4307$ & $\mathrm{POP}$ & 184 & 2016.664 & 241.6 & 0.2 & 11.612 & 0.039 & 1 & \\
\hline $21543+1943$ & STF & $2841 \mathrm{~A}, \mathrm{BC}$ & 2016.662 & 109.8 & 0.2 & 22.519 & 0.077 & 1 & \\
\hline $21543-2020$ & BVD & 140 & 2016.656 & 256.2 & 0.2 & 35.912 & 0.122 & 1 & \\
\hline $21547+4916$ & ES & 525 & 2016.683 & 122.8 & 0.2 & 5.252 & 0.018 & 1 & \\
\hline $21548+4548$ & STF & $2846 \mathrm{AB}$ & 2016.683 & 265.2 & 0.2 & 3.523 & 0.012 & 1 & \\
\hline $21548+4548$ & STF & $2846 \mathrm{AC}$ & 2016.683 & 155.9 & 0.2 & 33.193 & 0.113 & 1 & \\
\hline $21550-1158$ & HJ & 616 & 2016.656 & 275.0 & 0.2 & 30.499 & 0.104 & 1 & \\
\hline $21551+2523$ & $\mathrm{AG}$ & 421 & 2016.757 & 199.3 & 0.2 & 6.617 & 0.022 & 1 & \\
\hline $21552-1012$ & HJ & 949 & 2016.656 & 300.9 & 0.2 & 12.161 & 0.041 & 1 & \\
\hline $21554+3217$ & $\mathrm{~J}$ & 203 & 2016.757 & 68.0 & 0.2 & 4.401 & 0.015 & 1 & \\
\hline $21555+5232$ & STT & $456 \mathrm{AB}$ & 2016.683 & 37.4 & 0.2 & 1.622 & 0.006 & 1 & \\
\hline $21555+5232$ & STT & $456 \mathrm{AD}$ & 2016.683 & 186.0 & 0.2 & 25.153 & 0.086 & 1 & \\
\hline $21564+2335$ & HJ & 1708 & 2016.757 & 236.7 & 0.2 & 7.239 & 0.025 & 1 & \\
\hline $21568+5558$ & $\mathrm{~A}$ & 1897 & 2016.683 & 73.6 & 0.2 & 0.956 & 0.003 & 1 & \\
\hline $21573+0825$ & HJ & 5523 & 2016.659 & 205.1 & 0.2 & 20.438 & 0.069 & 1 & \\
\hline $21576+3433$ & $\mathrm{~J}$ & 3153 & 2016.757 & 231.6 & 0.2 & 8.332 & 0.028 & 1 & \\
\hline $21576+4004$ & SEI & 1546 & 2016.664 & 212.9 & 0.2 & 29.006 & 0.099 & 1 & \\
\hline $21576+4452$ & SMA & 153 & 2016.664 & 26.6 & 0.2 & 10.214 & 0.035 & 1 & \\
\hline $21582+4428$ & SMA & 154 & 2016.664 & 162.1 & 0.2 & 11.081 & 0.038 & 1 & \\
\hline $21583-0150$ & HJ & 3074 & 2016.656 & 297.8 & 0.2 & 2.178 & 0.007 & 1 & \\
\hline $21584+4423$ & LYS & 11 & 2016.664 & 56.0 & 0.2 & 12.070 & 0.041 & 1 & \\
\hline $21586+4442$ & ES & 683 & 2016.664 & 249.2 & 0.2 & 9.267 & 0.032 & 1 & \\
\hline
\end{tabular}


Table 4-Continued

\begin{tabular}{|c|c|c|c|c|c|c|c|c|c|}
\hline $\begin{array}{l}\text { WDS Desig. } \\
\alpha, \delta(2000)\end{array}$ & & $\begin{array}{l}\text { iscoverer } \\
\text { signation }\end{array}$ & JY & $\begin{array}{c}\theta \\
(0)\end{array}$ & $\begin{array}{l}\sigma \theta \\
(0)\end{array}$ & $\begin{array}{c}\rho \\
\left({ }^{\prime \prime}\right)\end{array}$ & $\begin{array}{l}\sigma \rho \\
\left({ }^{\prime \prime}\right)\end{array}$ & $\mathrm{n}$ & Note \\
\hline $21587+6434$ & HJ & 1713 & 2016.703 & 128.0 & 0.2 & 18.790 & 0.064 & 1 & \\
\hline $21588+4619$ & $\mathrm{UC}$ & 267 & 2016.683 & 246.7 & 0.2 & 17.191 & 0.058 & 1 & \\
\hline $21590+5511$ & STI & 2604 & 2016.683 & 7.1 & 0.2 & 11.596 & 0.039 & 1 & \\
\hline $21591+0116$ & HJ & 3078 & 2016.656 & 180.9 & 0.2 & 4.437 & 0.015 & 1 & \\
\hline $21595+0617$ & HJ & 3079 & 2016.659 & 74.4 & 0.2 & 9.858 & 0.034 & 1 & \\
\hline $21595+5037$ & HJ & 1710 & 2016.683 & 285.1 & 0.2 & 12.207 & 0.042 & 1 & \\
\hline $22003+3228$ & ES & 2387 & 2016.757 & 325.4 & 0.2 & 5.946 & 0.020 & 1 & \\
\hline $22006+5411$ & STF & 2852 & 2016.683 & 172.3 & 0.2 & 7.670 & 0.026 & 1 & \\
\hline $22009-2021$ & I & 675 & 2016.656 & 63.4 & 0.2 & 5.702 & 0.019 & 1 & \\
\hline $22009+6250$ & $\mathrm{HU}$ & 976 & 2016.700 & 57.3 & 0.2 & 1.631 & 0.006 & 1 & \\
\hline $22013+4515$ & $\mathrm{~A}$ & $780 \mathrm{AB}$ & 2016.664 & 148.4 & 0.2 & 1.541 & 0.005 & 1 & \\
\hline $22013+4515$ & $\mathrm{~A}$ & $780 \mathrm{AC}$ & 2016.664 & 94.6 & 0.2 & 66.968 & 0.228 & 1 & \\
\hline $22013+4515$ & $\mathrm{~A}$ & $780 \mathrm{CR}$ & 2016.664 & 27.4 & 0.2 & 16.842 & 0.057 & 1 & \\
\hline $22013+4621$ & HJ & 1714 & 2016.683 & 251.6 & 0.2 & 17.183 & 0.058 & 1 & \\
\hline $22024-1658$ & $\mathrm{~S}$ & $802 \mathrm{AB}$ & 2016.656 & 247.3 & 0.2 & 3.839 & 0.013 & 1 & \\
\hline $22024-1930$ & ARA & 812 & 2016.656 & 288.6 & 0.2 & 14.768 & 0.050 & 1 & \\
\hline $22028+0345$ & KU & 134 & 2016.659 & 260.5 & 0.2 & 55.622 & 0.189 & 1 & \\
\hline $22029+3934$ & STT & 459 & 2016.757 & 195.7 & 0.2 & 10.712 & 0.036 & 1 & \\
\hline $22048+5125$ & HJ & 1724 & 2016.785 & 225.8 & 0.2 & 13.426 & 0.046 & 1 & \\
\hline $22049+1107$ & DOO & 89 & 2016.662 & 327.4 & 0.2 & 8.772 & 0.030 & 1 & \\
\hline $22053+4629$ & HJ & 1725 & 2016.839 & 78.6 & 0.2 & 29.851 & 0.101 & 1 & \\
\hline $22055+4007$ & MLB & 31 & 2016.853 & 306.0 & 0.2 & 9.986 & 0.034 & 1 & \\
\hline $22057+5819$ & HJ & 1729 & 2016.703 & 98.6 & 0.2 & 7.683 & 0.026 & 1 & \\
\hline $22058+0452$ & STF & 2856 & 2016.662 & 194.1 & 0.2 & 1.252 & 0.004 & 1 & \\
\hline $22060+2048$ & STF & 2861 & 2016.815 & 220.6 & 0.2 & 7.141 & 0.024 & 1 & \\
\hline $22064+4716$ & $\mathrm{ES}$ & 532 & 2016.839 & 242.2 & 0.2 & 9.875 & 0.034 & 1 & \\
\hline $22066+5346$ & HJ & 3093 & 2016.703 & 13.0 & 0.2 & 10.352 & 0.035 & 1 & \\
\hline $22069+4752$ & $\mathrm{ES}$ & 2715 & 2016.839 & 279.8 & 0.2 & 36.833 & 0.125 & 1 & \\
\hline $22071+0034$ & STF & 2862 & 2016.662 & 95.5 & 0.2 & 2.520 & 0.009 & 1 & \\
\hline $22075+5024$ & HJ & 1732 & 2016.785 & 247.8 & 0.2 & 9.382 & 0.032 & 1 & \\
\hline $22081+3954$ & MLB & 903 & 2016.853 & 197.3 & 0.2 & 4.983 & 0.017 & 1 & \\
\hline $22086+5917$ & STF & $2872 \mathrm{~A}, \mathrm{BC}$ & 2016.703 & 315.8 & 0.2 & 21.715 & 0.074 & 1 & \\
\hline
\end{tabular}


Table 4-Continued

\begin{tabular}{|c|c|c|c|c|c|c|c|c|c|}
\hline $\begin{array}{l}\text { WDS Desig. } \\
\alpha, \delta(2000)\end{array}$ & & $\begin{array}{l}\text { iscoverer } \\
\text { signation }\end{array}$ & JY & $\begin{array}{c}\theta \\
(0)\end{array}$ & $\begin{array}{l}\sigma \theta \\
(0)\end{array}$ & $\begin{array}{c}\rho \\
\left({ }^{\prime \prime}\right)\end{array}$ & $\begin{array}{l}\sigma \rho \\
\left({ }^{\prime \prime}\right)\end{array}$ & $\mathrm{n}$ & Note \\
\hline $22093-1859$ & $\mathrm{HU}$ & 284 & 2016.727 & 114.2 & 0.2 & 3.457 & 0.012 & 1 & \\
\hline $22093+4451$ & HJ & $1735 \mathrm{AB}$ & 2016.839 & 110.3 & 0.2 & 27.210 & 0.093 & 1 & \\
\hline $22094+2233$ & STF & 2868 & 2016.815 & 352.2 & 0.2 & 1.081 & 0.004 & 1 & \\
\hline $22095-1827$ & HJ & 3092 & 2016.727 & 342.3 & 0.2 & 28.563 & 0.097 & 1 & \\
\hline $22100+2203$ & $\mathrm{COU}$ & $137 \mathrm{AC}$ & 2016.815 & 231.5 & 0.2 & 24.612 & 0.084 & 1 & \\
\hline $22104+1925$ & BRT & 2527 & 2016.815 & 10.9 & 0.2 & 5.661 & 0.019 & 1 & \\
\hline $22115+4232$ & GRV & 545 & 2016.839 & 254.1 & 0.2 & 22.320 & 0.076 & 1 & \\
\hline $22115+6703$ & MLB & 1099 & 2016.703 & 213.4 & 0.2 & 11.264 & 0.038 & 1 & \\
\hline $22116+5425$ & MCK & 6 & 2016.703 & 136.9 & 0.2 & 12.208 & 0.042 & 1 & \\
\hline $22117+5743$ & A & $625 \mathrm{AB}$ & 2016.703 & 79.0 & 0.2 & 0.507 & 0.002 & 1 & \\
\hline $22117+5743$ & A & $625 \mathrm{AB}, \mathrm{C}$ & 2016.703 & 13.7 & 0.2 & 30.455 & 0.104 & 1 & \\
\hline $22118+4711$ & BRT & 1158 & 2016.839 & 147.9 & 0.2 & 5.325 & 0.018 & 1 & \\
\hline $22127-2006$ & STN & 57 & 2016.727 & 97.0 & 0.2 & 10.039 & 0.034 & 1 & \\
\hline $22128+1355$ & $\mathrm{HU}$ & 978 & 2016.662 & 205.5 & 0.2 & 1.202 & 0.004 & 1 & \\
\hline $22129+7318$ & STF & 2893 & 2016.700 & 347.1 & 0.2 & 29.001 & 0.099 & 1 & \\
\hline $22132+2148$ & HJ & 958 & 2016.815 & 232.2 & 0.2 & 5.862 & 0.020 & 1 & \\
\hline $22133+3207$ & ES & 388 & 2016.891 & 264.1 & 0.2 & 7.690 & 0.026 & 1 & \\
\hline $22138+4520$ & BRT & 1159 & 2016.839 & 234.3 & 0.2 & 4.455 & 0.015 & 1 & \\
\hline $22141+3123$ & $\mathrm{BU}$ & 476 & 2016.815 & 92.8 & 0.2 & 2.847 & 0.010 & 1 & \\
\hline $22142+6128$ & ES & 1924 & 2016.703 & 144.7 & 0.2 & 5.894 & 0.020 & 1 & \\
\hline $22143+3745$ & STF & 2882 & 2016.853 & 148.6 & 0.2 & 3.444 & 0.012 & 1 & \\
\hline $22143+6155$ & FOX & 265 & 2016.703 & 175.8 & 0.2 & 5.527 & 0.019 & 1 & \\
\hline $22146+2934$ & STF & 2881 & 2016.815 & 75.6 & 0.2 & 1.298 & 0.004 & 1 & \\
\hline $22147+4921$ & STF & $2886 \mathrm{AB}$ & 2016.839 & 108.4 & 0.2 & 20.822 & 0.071 & 1 & \\
\hline $22152+4953$ & STF & $2890 \mathrm{AB}$ & 2016.839 & 11.4 & 0.2 & 9.454 & 0.032 & 1 & \\
\hline $22154+5104$ & $\mathrm{ACA}$ & $14 \mathrm{AC}$ & 2016.703 & 328.7 & 0.2 & 16.505 & 0.056 & 1 & \\
\hline $22154+5104$ & $\mathrm{ACA}$ & $14 \mathrm{AD}$ & 2016.703 & 26.6 & 0.2 & 22.914 & 0.078 & 1 & \\
\hline $22154+5104$ & ES & $1113 \mathrm{AB}$ & 2016.703 & 220.5 & 0.2 & 7.107 & 0.024 & 1 & \\
\hline $22159+3125$ & $\mathrm{BU}$ & 477 & 2016.815 & 41.6 & 0.2 & 6.427 & 0.022 & 1 & \\
\hline $22165+0841$ & $\mathrm{~J}$ & 2403 & 2016.826 & 93.4 & 0.2 & 5.435 & 0.018 & 1 & \\
\hline $22166+3939$ & ALI & 1171 & 2016.839 & 215.8 & 0.2 & 13.969 & 0.047 & 1 & \\
\hline $22167-1112$ & HJ & $3100 \mathrm{AB}$ & 2016.727 & 75.3 & 0.2 & 39.473 & 0.134 & 1 & \\
\hline
\end{tabular}


Table 4-Continued

\begin{tabular}{|c|c|c|c|c|c|c|c|c|c|}
\hline $\begin{array}{l}\text { WDS Desig. } \\
\alpha, \delta(2000)\end{array}$ & $\begin{array}{l}\text { Dis } \\
\text { Des }\end{array}$ & $\begin{array}{l}\text { scoverer } \\
\text { ignation }\end{array}$ & JY & $\begin{array}{c}\theta \\
(0)\end{array}$ & $\begin{array}{l}\sigma \theta \\
(0)\end{array}$ & $\begin{array}{c}\rho \\
\left({ }^{\prime \prime}\right)\end{array}$ & $\begin{array}{l}\sigma \rho \\
\left({ }^{\prime \prime}\right)\end{array}$ & $\mathrm{n}$ & Note \\
\hline $22173-0042$ & STF & 2887 & 2016.662 & 30.4 & 0.2 & 7.835 & 0.027 & 1 & \\
\hline $22184+4940$ & ES & $534 \mathrm{AC}$ & 2016.839 & 69.3 & 0.2 & 47.520 & 0.162 & 1 & \\
\hline $22184+4940$ & ES & $534 \mathrm{CD}$ & 2016.839 & 103.5 & 0.2 & 7.167 & 0.024 & 1 & \\
\hline $22184+5201$ & ES & 1114 & 2016.703 & 256.6 & 0.2 & 6.282 & 0.021 & 1 & \\
\hline $22189+3746$ & STF & $2894 \mathrm{AB}$ & 2016.839 & 194.3 & 0.2 & 15.983 & 0.054 & 1 & \\
\hline 22191-1706 & HJ & 3104 & 2016.727 & 69.8 & 0.2 & 10.909 & 0.037 & 1 & \\
\hline $22193-1047$ & STF & $2892 \mathrm{AB}$ & 2016.662 & 56.6 & 0.2 & 11.595 & 0.039 & 1 & \\
\hline $22193-1047$ & STF & $2892 \mathrm{AC}$ & 2016.662 & 258.4 & 0.2 & 38.682 & 0.132 & 1 & \\
\hline $22193+7450$ & HJ & 1761 & 2016.700 & 34.8 & 0.2 & 4.807 & 0.016 & 1 & \\
\hline $22204+4514$ & HJ & $1753 \mathrm{AB}$ & 2016.839 & 182.7 & 0.2 & 5.013 & 0.017 & 1 & \\
\hline $22204+4514$ & HJ & $1753 \mathrm{AC}$ & 2016.839 & 175.6 & 0.2 & 42.191 & 0.143 & 1 & \\
\hline $22206+5349$ & $\mathrm{BU}$ & 379 & 2016.785 & 334.0 & 0.2 & 1.131 & 0.004 & 1 & \\
\hline $22210-0334$ & HJ & 5329 & 2016.727 & 98.3 & 0.2 & 8.131 & 0.028 & 1 & \\
\hline $22211+5428$ & ES & 2718 & 2016.785 & 84.1 & 0.2 & 20.320 & 0.069 & 1 & \\
\hline $22218+1515$ & $\mathrm{STF}$ & 2897 & 2016.757 & 98.6 & 0.2 & 16.949 & 0.058 & 1 & \\
\hline $22220-2050$ & HDS & 3169 & 2016.727 & 202.5 & 0.2 & 15.191 & 0.052 & 1 & \\
\hline $22232+7054$ & A & 783 & 2016.700 & 181.2 & 0.2 & 4.523 & 0.015 & 1 & \\
\hline $22234+4824$ & HJ & $1762 \mathrm{AB}$ & 2016.779 & 355.0 & 0.2 & 18.422 & 0.063 & 1 & \\
\hline $22236+4521$ & STF & $2902 \mathrm{AB}$ & 2016.779 & 89.1 & 0.2 & 6.517 & 0.022 & 1 & \\
\hline $22236+5431$ & ES & 1021 & 2016.785 & 106.3 & 0.2 & 4.713 & 0.016 & 1 & \\
\hline $22242+2712$ & HJ & 1760 & 2016.760 & 335.9 & 0.2 & 6.973 & 0.024 & 1 & \\
\hline $22244+3648$ & $\mathrm{~J}$ & $3166 \mathrm{AB}$ & 2016.891 & 177.1 & 0.2 & 4.055 & 0.014 & 1 & \\
\hline $22244+3648$ & $\mathrm{~J}$ & $3166 \mathrm{AC}$ & 2016.891 & 145.5 & 0.2 & 23.522 & 0.080 & 1 & \\
\hline $22245+4720$ & ES & 1281 & 2016.779 & 170.2 & 0.2 & 5.808 & 0.020 & 1 & \\
\hline $22245+5452$ & ES & $147 \mathrm{AB}$ & 2016.785 & 24.9 & 0.2 & 2.614 & 0.009 & 1 & \\
\hline $22245+5452$ & ES & $147 \mathrm{AC}$ & 2016.785 & 204.3 & 0.2 & 31.190 & 0.106 & 1 & \\
\hline $22254+1842$ & HJ & 963 & 2016.757 & 59.7 & 0.2 & 7.154 & 0.024 & 1 & \\
\hline $22256+4807$ & ES & 687 & 2016.779 & 266.6 & 0.2 & 4.370 & 0.015 & 1 & \\
\hline $22261-1225$ & HJ & $3113 \mathrm{AB}$ & 2016.727 & 182.1 & 0.2 & 13.402 & 0.046 & 1 & \\
\hline $22261-1225$ & HJ & $3113 \mathrm{AC}$ & 2016.727 & 315.1 & 0.2 & 24.769 & 0.084 & 1 & \\
\hline $22265+4332$ & $\mathrm{HO}$ & $184 \mathrm{AB}$ & 2016.779 & 292.5 & 0.2 & 2.634 & 0.009 & 1 & \\
\hline $22265+4332$ & $\mathrm{HO}$ & $184 \mathrm{AC}$ & 2016.779 & 315.6 & 0.2 & 44.264 & 0.150 & 1 & \\
\hline
\end{tabular}


Table 4-Continued

\begin{tabular}{|c|c|c|c|c|c|c|c|c|c|}
\hline \multirow{2}{*}{$\begin{array}{c}\text { WDS Desig. } \\
\alpha, \delta(2000) \\
22266+5019\end{array}$} & \multicolumn{2}{|c|}{$\begin{array}{c}\text { Discoverer } \\
\text { Designation }\end{array}$} & \multirow{2}{*}{$\begin{array}{c}\text { JY } \\
2016.779\end{array}$} & \multirow{2}{*}{$\begin{array}{c}\theta \\
(\circ) \\
270.1\end{array}$} & \multirow{2}{*}{$\begin{array}{l}\sigma \theta \\
(\circ) \\
0.2\end{array}$} & \multirow{2}{*}{$\begin{array}{c}\rho \\
\left({ }^{\prime \prime}\right) \\
14.495\end{array}$} & \multirow{2}{*}{$\begin{array}{c}\sigma \rho \\
\left({ }^{\prime \prime}\right) \\
0.049\end{array}$} & \multirow{2}{*}{$\begin{array}{l}\mathrm{n} \\
\mathrm{1} \\
1\end{array}$} & \multirow{2}{*}{$\begin{array}{l}\text { Note } \\
\end{array}$} \\
\hline & HJ & $1766 \mathrm{AB}$ & & & & & & & \\
\hline $22269+6343$ & KR & 59 & 2016.785 & 345.1 & 0.2 & 1.814 & 0.006 & 1 & \\
\hline $22272-0146$ & STF & 2904 & 2016.727 & 311.4 & 0.2 & 8.304 & 0.028 & 1 & \\
\hline $22272+1509$ & STF & 2905 & 2016.757 & 284.8 & 0.2 & 3.328 & 0.011 & 1 & \\
\hline $22274+4749$ & HJ & 1768 & 2016.779 & 8.3 & 0.2 & 24.635 & 0.084 & 1 & \\
\hline $22292+5825$ & STFA & $58 \mathrm{AC}$ & 2016.785 & 191.5 & 0.2 & 40.883 & 0.139 & 1 & \\
\hline $22294-0720$ & $\mathrm{BU}$ & $478 \mathrm{AB}$ & 2016.727 & 40.7 & 0.2 & 1.689 & 0.006 & 1 & \\
\hline $22294-0720$ & $\mathrm{BU}$ & $478 \mathrm{AC}$ & 2016.727 & 238.5 & 0.2 & 29.092 & 0.099 & 1 & \\
\hline $22294+3916$ & MLB & 796 & 2016.703 & 317.5 & 0.2 & 5.809 & 0.020 & 1 & \\
\hline $22295+8050$ & $\mathrm{STF}$ & 2927 & 2016.700 & 314.8 & 0.2 & 15.680 & 0.053 & 1 & \\
\hline $22306+5332$ & $\mathrm{STF}$ & $2917 \mathrm{AB}$ & 2016.779 & 70.4 & 0.2 & 4.756 & 0.016 & 1 & \\
\hline $22307+3729$ & ES & $2072 \mathrm{AB}$ & 2016.891 & 326.3 & 0.2 & 13.424 & 0.046 & 1 & \\
\hline $22309+4826$ & HJ & 1777 & 2016.779 & 315.1 & 0.2 & 12.059 & 0.041 & 1 & \\
\hline $22318+5004$ & ES & $837 \mathrm{AD}$ & 2016.779 & 171.2 & 0.2 & 44.669 & 0.152 & 1 & \\
\hline $22323+5156$ & ES & 1116 & 2016.779 & 133.7 & 0.2 & 7.196 & 0.024 & 1 & \\
\hline $22323+5512$ & AG & 283 & 2016.785 & 332.0 & 0.2 & 2.666 & 0.009 & 1 & \\
\hline $22324+6015$ & HJ & $1783 \mathrm{CD}$ & 2016.785 & 274.7 & 0.2 & 6.305 & 0.021 & 1 & \\
\hline $22328+2625$ & $\mathrm{HO}$ & $475 \mathrm{AB}$ & 2016.757 & 306.2 & 0.2 & 1.107 & 0.004 & 1 & \\
\hline $22328+2625$ & $\mathrm{HO}$ & $475 \mathrm{AC}$ & 2016.757 & 223.5 & 0.2 & 8.388 & 0.029 & 1 & \\
\hline $22339+2635$ & $\mathrm{HO}$ & 476 & 2016.757 & 207.2 & 0.2 & 6.919 & 0.024 & 1 & \\
\hline $22340-0147$ & $\mathrm{BU}$ & $77 \mathrm{AB}$ & 2016.727 & 214.8 & 0.2 & 2.875 & 0.010 & 1 & \\
\hline $22342+4341$ & ES & 1468 & 2016.760 & 326.7 & 0.2 & 5.992 & 0.020 & 1 & \\
\hline $22343+5023$ & ARG & 44 & 2016.779 & 167.7 & 0.2 & 7.413 & 0.025 & 1 & \\
\hline $22345+4046$ & HJ & $1786 \mathrm{AB}$ & 2016.703 & 225.8 & 0.2 & 44.198 & 0.150 & 1 & \\
\hline $22349+4134$ & HJ & 1788 & 2016.760 & 299.5 & 0.2 & 3.702 & 0.013 & 1 & \\
\hline $22352+5502$ & HJ & $1789 \mathrm{AB}$ & 2016.785 & 105.8 & 0.2 & 16.616 & 0.056 & 1 & \\
\hline $22352+5502$ & HJ & $1789 \mathrm{AC}$ & 2016.785 & 189.0 & 0.2 & 18.145 & 0.062 & 1 & \\
\hline $22355+0234$ & HLD & $51 \mathrm{AB}$ & 2016.727 & 188.1 & 0.2 & 1.161 & 0.004 & 1 & \\
\hline $22357+5652$ & HJ & $1791 \mathrm{AB}$ & 2016.785 & 59.4 & 0.2 & 17.120 & 0.058 & 1 & \\
\hline $22359+3938$ & $\mathrm{~A}$ & $1469 \mathrm{AC}$ & 2016.703 & 168.1 & 0.2 & 48.813 & 0.166 & 1 & \\
\hline $22359+3938$ & A & $1469 \mathrm{AD}$ & 2016.703 & 143.9 & 0.2 & 81.888 & 0.278 & 1 & \\
\hline $22359+3938$ & $\mathrm{~A}$ & $1469 \mathrm{BD}$ & 2016.703 & 131.1 & 0.2 & 66.863 & 0.227 & 1 & \\
\hline
\end{tabular}


Table 4-Continued

\begin{tabular}{|c|c|c|c|c|c|c|c|c|c|}
\hline $\begin{array}{l}\text { WDS Desig. } \\
\alpha, \delta(2000)\end{array}$ & & $\begin{array}{l}\text { iscoverer } \\
\text { esignation }\end{array}$ & JY & $\begin{array}{c}\theta \\
(0)\end{array}$ & $\begin{array}{l}\sigma \theta \\
(\circ)\end{array}$ & $\begin{array}{c}\rho \\
\left({ }^{\prime \prime}\right)\end{array}$ & $\begin{array}{l}\sigma \rho \\
\left({ }^{\prime \prime}\right)\end{array}$ & $\mathrm{n}$ & Note \\
\hline $22359+3938$ & $\mathrm{~A}$ & 1469 CD & 2016.703 & 115.9 & 0.2 & 42.365 & 0.144 & 1 & \\
\hline $22359+3938$ & STF & $2922 \mathrm{AB}$ & 2016.703 & 185.5 & 0.2 & 22.416 & 0.076 & 1 & \\
\hline $22359+3938$ & STF & $2922 \mathrm{BC}$ & 2016.703 & 154.3 & 0.2 & 28.312 & 0.096 & 1 & \\
\hline $22361+7253$ & STT & $\mathrm{A} 236 \mathrm{AB}, \mathrm{D}$ & 2016.700 & 136.9 & 0.2 & 42.376 & 0.144 & 1 & \\
\hline $22368+2732$ & MLB & $584 \mathrm{AB}$ & 2016.918 & 311.9 & 0.2 & 5.086 & 0.017 & 1 & \\
\hline $22372+5414$ & HJ & 3127 & 2016.779 & 297.7 & 0.2 & 7.565 & 0.026 & 1 & \\
\hline $22379+0554$ & STF & 2925 & 2016.727 & 3.7 & 0.2 & 7.213 & 0.025 & 1 & \\
\hline $22384+5223$ & BAR & $61 \mathrm{AB}$ & 2016.779 & 166.2 & 0.2 & 29.170 & 0.099 & 1 & \\
\hline $22384+5223$ & BAR & $61 \mathrm{BC}$ & 2016.779 & 181.6 & 0.2 & 2.703 & 0.009 & 1 & $\mathrm{C}$ \\
\hline $22384+5223$ & ES & $2720 \mathrm{BD}$ & 2016.779 & 244.7 & 0.2 & 13.338 & 0.045 & 1 & \\
\hline $22395+3653$ & HJ & 968 & 2016.760 & 110.5 & 0.2 & 4.361 & 0.015 & 1 & \\
\hline $22396+5007$ & HJ & 1797 & 2016.850 & 125.3 & 0.2 & 15.144 & 0.051 & 1 & \\
\hline $22398+4843$ & $\mathrm{ES}$ & 843 & 2016.853 & 207.5 & 0.2 & 8.214 & 0.028 & 1 & \\
\hline $22413+1311$ & STF & $2931 \mathrm{AB}$ & 2016.827 & 149.2 & 0.1 & 4.463 & 0.010 & 3 & \\
\hline $22413+7244$ & STF & 2940 & 2016.700 & 138.4 & 0.2 & 2.715 & 0.009 & 1 & \\
\hline $22415+3256$ & $\mathrm{~J}$ & $2376 \mathrm{AB}$ & 2016.883 & 195.6 & 0.2 & 7.214 & 0.025 & 1 & \\
\hline $22425+3917$ & $\mathrm{BU}$ & 176 & 2016.883 & 55.1 & 0.2 & 2.573 & 0.009 & 1 & \\
\hline $22426+4401$ & A & $414 \mathrm{AB}$ & 2016.880 & 14.5 & 0.2 & 1.739 & 0.006 & 1 & \\
\hline $22426+4401$ & A & $414 \mathrm{AC}$ & 2016.880 & 84.2 & 0.2 & 25.529 & 0.087 & 1 & \\
\hline $22435-1608$ & $\mathrm{HU}$ & 289 & 2016.826 & 280.5 & 0.2 & 1.327 & 0.005 & 1 & \\
\hline $22435+3813$ & $\mathrm{ACA}$ & $13 \mathrm{AC}$ & 2016.883 & 107.6 & 0.2 & 53.999 & 0.184 & 1 & \\
\hline $22435+3813$ & DOB & $16 \mathrm{AB}$ & 2016.883 & 79.1 & 0.2 & 23.321 & 0.079 & 1 & \\
\hline $22437+4015$ & MLB & 995 & 2016.880 & 303.7 & 0.2 & 4.719 & 0.016 & 1 & \\
\hline $22439+3337$ & $\mathrm{ES}$ & 265 & 2016.909 & 5.7 & 0.0 & 8.647 & 0.016 & 2 & \\
\hline $22442+4105$ & $\mathrm{~J}$ & 857 & 2016.880 & 321.7 & 0.2 & 3.209 & 0.011 & 1 & \\
\hline $22443+3442$ & $\mathrm{ES}$ & 2203 & 2016.883 & 97.1 & 0.2 & 2.115 & 0.007 & 1 & \\
\hline $22445+3717$ & ALI & 461 & 2016.883 & 1.3 & 0.2 & 10.543 & 0.036 & 1 & \\
\hline $22448-0359$ & HJ & $5359 \mathrm{AB}$ & 2016.807 & 64.9 & 0.2 & 20.484 & 0.035 & 2 & \\
\hline $22448-0359$ & HJ & $5359 \mathrm{AC}$ & 2016.793 & 356.7 & 0.3 & 15.561 & 0.006 & 2 & \\
\hline $22448+4700$ & HJ & 1805 & 2016.853 & 157.6 & 0.2 & 7.344 & 0.025 & 1 & \\
\hline $22450+4928$ & SAN & 3 & 2016.853 & 321.4 & 0.2 & 6.237 & 0.021 & 1 & \\
\hline $22450+6808$ & STT & $529 \mathrm{AB}$ & 2016.850 & 201.5 & 0.2 & 3.668 & 0.012 & 1 & \\
\hline
\end{tabular}


Table 4-Continued

\begin{tabular}{|c|c|c|c|c|c|c|c|c|c|}
\hline $\begin{array}{l}\text { WDS Desig. } \\
\alpha, \delta(2000)\end{array}$ & $\begin{array}{l}\text { Dis } \\
\text { Des }\end{array}$ & $\begin{array}{l}\text { scoverer } \\
\text { ignation }\end{array}$ & JY & $\begin{array}{c}\theta \\
(0)\end{array}$ & $\begin{array}{l}\sigma \theta \\
(\circ)\end{array}$ & $\begin{array}{c}\rho \\
\left({ }^{\prime \prime}\right)\end{array}$ & $\begin{array}{l}\sigma \rho \\
\left({ }^{\prime \prime}\right)\end{array}$ & $\mathrm{n}$ & Note \\
\hline $22450+6808$ & STT & $529 \mathrm{AC}$ & 2016.850 & 223.5 & 0.2 & 17.173 & 0.058 & 1 & \\
\hline $22451+4449$ & HJ & 1806 & 2016.880 & 334.8 & 0.2 & 7.127 & 0.024 & 1 & \\
\hline $22453+4002$ & $\mathrm{AG}$ & 287 & 2016.880 & 194.2 & 0.2 & 14.469 & 0.049 & 1 & \\
\hline $22455+3359$ & $\mathrm{HU}$ & 782 & 2016.935 & 322.6 & 0.2 & 1.990 & 0.007 & 1 & \\
\hline $22459+3358$ & HJ & 969 & 2016.886 & 26.4 & 0.1 & 5.938 & 0.034 & 2 & \\
\hline $22460-1536$ & $\mathrm{HU}$ & 290 & 2016.757 & 353.5 & 0.2 & 17.529 & 0.060 & 1 & \\
\hline $22470+4446$ & A & $189 \mathrm{AB}$ & 2016.880 & 26.6 & 0.2 & 1.004 & 0.003 & 1 & \\
\hline $22485+5409$ & $A G$ & 424 & 2016.850 & 136.7 & 0.2 & 2.265 & 0.008 & 1 & \\
\hline $22486+4136$ & HJ & 1813 & 2016.880 & 61.8 & 0.2 & 9.438 & 0.032 & 1 & \\
\hline $22487+3818$ & $\mathrm{AG}$ & 288 & 2016.883 & 187.0 & 0.2 & 18.436 & 0.063 & 1 & \\
\hline $22494+4528$ & HJ & 1815 & 2016.853 & 31.6 & 0.2 & 10.051 & 0.034 & 1 & \\
\hline $22496-1059$ & $\mathrm{BU}$ & 1518 & 2016.757 & 204.2 & 0.2 & 6.378 & 0.022 & 1 & \\
\hline $22497+3119$ & STF & 2945 & 2016.883 & 300.4 & 0.2 & 4.226 & 0.014 & 1 & \\
\hline $22497+4031$ & STF & 2946 & 2016.880 & 262.6 & 0.2 & 5.482 & 0.019 & 1 & \\
\hline $22499+4620$ & HJ & 1816 & 2016.853 & 134.8 & 0.2 & 6.962 & 0.024 & 1 & \\
\hline $22500+6018$ & HJ & 1821 & 2016.850 & 103.4 & 0.2 & 11.584 & 0.039 & 1 & \\
\hline $22505+3258$ & $\mathrm{~J}$ & 3182 & 2016.884 & 158.6 & 0.1 & 4.083 & 0.036 & 2 & \\
\hline $22506+5306$ & $\mathrm{BU}$ & $1519 \mathrm{AB}$ & 2016.850 & 176.7 & 0.2 & 4.467 & 0.015 & 1 & \\
\hline $22506+5306$ & HJ & $5548 \mathrm{AC}$ & 2016.850 & 226.2 & 0.2 & 27.577 & 0.094 & 1 & \\
\hline $22507+5107$ & HLD & 54 & 2016.850 & 17.9 & 0.2 & 1.884 & 0.006 & 1 & \\
\hline $22509+5303$ & $\mathrm{BU}$ & $1332 \mathrm{AB}$ & 2016.850 & 129.7 & 0.2 & 1.494 & 0.005 & 1 & \\
\hline $22512+5712$ & HJ & 1824 & 2016.850 & 44.9 & 0.2 & 22.508 & 0.077 & 1 & \\
\hline $22518+4804$ & $\mathrm{ES}$ & 851 & 2016.853 & 264.4 & 0.2 & 8.699 & 0.030 & 1 & \\
\hline $22519+5304$ & HJ & 3150 & 2016.850 & 291.2 & 0.2 & 20.273 & 0.069 & 1 & \\
\hline $22522+4734$ & $\mathrm{ES}$ & 852 & 2016.853 & 327.8 & 0.2 & 7.273 & 0.025 & 1 & \\
\hline $22525+4828$ & HEI & 87 & 2016.850 & 280.5 & 0.2 & 5.226 & 0.018 & 1 & \\
\hline $22540+4000$ & $\mathrm{ES}$ & 1701 & 2016.880 & 65.9 & 0.2 & 8.255 & 0.028 & 1 & \\
\hline $22542+7620$ & STF & 2963 & 2016.700 & 3.0 & 0.2 & 1.903 & 0.006 & 1 & \\
\hline $22544+5538$ & HJ & $1830 \mathrm{AC}$ & 2016.850 & 83.3 & 0.2 & 13.559 & 0.046 & 1 & \\
\hline $22546+2020$ & $\mathrm{BU}$ & 847 & 2016.900 & 36.1 & 0.1 & 6.876 & 0.005 & 3 & \\
\hline $22548+5914$ & $\mathrm{BU}$ & $712 \mathrm{AC}$ & 2016.850 & 112.5 & 0.2 & 45.478 & 0.155 & 1 & \\
\hline $22555-0535$ & $\mathrm{BU}$ & 1010 & 2016.826 & 313.0 & 0.2 & 1.104 & 0.004 & 1 & \\
\hline
\end{tabular}


Table 4-Continued

\begin{tabular}{|c|c|c|c|c|c|c|c|c|c|}
\hline $\begin{array}{l}\text { WDS Desig. } \\
\alpha, \delta(2000)\end{array}$ & & $\begin{array}{l}\text { liscoverer } \\
\text { esignation }\end{array}$ & JY & $\begin{array}{c}\theta \\
(0)\end{array}$ & $\begin{array}{l}\sigma \theta \\
(0)\end{array}$ & $\begin{array}{c}\rho \\
\left({ }^{\prime \prime}\right)\end{array}$ & $\begin{array}{l}\sigma \rho \\
\left({ }^{\prime \prime}\right)\end{array}$ & $\mathrm{n}$ & Note \\
\hline $22555+3640$ & ES & 2721 & 2016.883 & 107.0 & 0.2 & 9.224 & 0.031 & 1 & \\
\hline $22557+4303$ & HJ & 1831 & 2016.880 & 92.7 & 0.2 & 14.496 & 0.049 & 1 & \\
\hline $22558-2018$ & ARA & 1243 & 2016.757 & 271.9 & 0.2 & 10.099 & 0.034 & 1 & \\
\hline $22558+4508$ & SMA & A 181 & 2016.850 & 281.1 & 0.2 & 9.730 & 0.033 & 1 & \\
\hline $22562+7250$ & STF & $2965 \mathrm{DE}$ & 2016.728 & 220.5 & 0.1 & 2.891 & 0.006 & 2 & \\
\hline $22562+7250$ & STT & $484 \mathrm{AB}, \mathrm{C}$ & 2016.839 & 255.8 & 0.2 & 30.753 & 0.105 & 1 & \\
\hline $22564+1727$ & STF & $2957 \mathrm{AB}$ & 2016.760 & 224.2 & 0.2 & 4.587 & 0.016 & 1 & \\
\hline $22565+3150$ & HJ & 976 & 2016.815 & 72.4 & 0.2 & 8.488 & 0.029 & 1 & \\
\hline $22565+6252$ & STF & 2961 & 2016.839 & 348.4 & 0.2 & 1.902 & 0.006 & 1 & \\
\hline $22567+7830$ & $\mathrm{STF}$ & 2971 & 2016.700 & 4.1 & 0.2 & 5.505 & 0.019 & 1 & \\
\hline $22569+1225$ & BRT & 1961 & 2016.967 & 302.5 & 0.7 & 4.753 & 0.003 & 2 & \\
\hline $22569+2352$ & $\mathrm{POU}$ & J 5763 & 2016.760 & 250.0 & 0.2 & 7.531 & 0.026 & 1 & \\
\hline $22578+3840$ & HJ & 1832 & 2016.817 & 73.7 & 0.2 & 11.354 & 0.039 & 1 & \\
\hline $22581+5647$ & MLE & 376 & 2016.839 & 241.3 & 0.2 & 7.662 & 0.026 & 1 & \\
\hline $22581+6322$ & BHJ & 44 & 2016.839 & 70.8 & 0.2 & 17.584 & 0.060 & 1 & \\
\hline $22582+1306$ & HJ & 3156 & 2016.928 & 295.0 & 0.3 & 23.056 & 0.005 & 2 & \\
\hline $22582+5133$ & ES & 1120 & 2016.817 & 27.6 & 0.2 & 8.947 & 0.030 & 1 & \\
\hline $22585+6705$ & HJ & 1838 & 2016.839 & 255.2 & 0.2 & 1.463 & 0.005 & 1 & \\
\hline $22590+2745$ & STF & 2967 & 2016.815 & 5.2 & 0.2 & 6.763 & 0.023 & 1 & \\
\hline $22590+7046$ & HJ & 3158 & 2016.700 & 211.9 & 0.2 & 1.009 & 0.003 & 1 & \\
\hline $22597+4149$ & HLD & 56 & 2016.817 & 91.9 & 0.2 & 1.194 & 0.004 & 1 & \\
\hline $23003+3714$ & $\mathrm{ES}$ & 2076 & 2016.817 & 161.8 & 0.2 & 1.794 & 0.006 & 1 & \\
\hline $23004+0353$ & $\mathrm{~J}$ & 1797 & 2016.730 & 144.5 & 0.2 & 4.201 & 0.014 & 1 & \\
\hline $23010+2646$ & STF & 2969 & 2016.815 & 36.0 & 0.2 & 3.989 & 0.014 & 1 & \\
\hline $23014+4638$ & $\mathrm{~A}$ & 193 & 2016.817 & 175.2 & 0.2 & 1.569 & 0.005 & 1 & \\
\hline $23017+2821$ & MLE & 3547 & 2016.815 & 268.9 & 0.2 & 4.693 & 0.016 & 1 & \\
\hline $23021+5455$ & ES & 2723 & 2016.839 & 252.7 & 0.2 & 31.113 & 0.106 & 1 & \\
\hline $23024-1119$ & STF & 2970 & 2016.730 & 38.5 & 0.2 & 8.517 & 0.029 & 1 & \\
\hline $23029+4610$ & HJ & $1841 \mathrm{AB}$ & 2016.817 & 344.2 & 0.2 & 18.308 & 0.062 & 1 & \\
\hline $23029+4610$ & HJ & $1841 \mathrm{AC}$ & 2016.817 & 285.7 & 0.2 & 33.821 & 0.115 & 1 & \\
\hline $23034+6027$ & STT & 486 & 2016.839 & 276.4 & 0.2 & 33.984 & 0.116 & 1 & \\
\hline $23042+4636$ & $\mathrm{ARC}$ & G 45 & 2016.817 & 16.1 & 0.2 & 3.774 & 0.013 & 1 & \\
\hline
\end{tabular}


Table 4-Continued

\begin{tabular}{|c|c|c|c|c|c|c|c|c|c|}
\hline $\begin{array}{l}\text { WDS Desig. } \\
\alpha, \delta(2000)\end{array}$ & $\begin{array}{l}\text { Dis } \\
\text { Des }\end{array}$ & $\begin{array}{l}\text { scoverer } \\
\text { ignation }\end{array}$ & JY & $\begin{array}{c}\theta \\
(0)\end{array}$ & $\begin{array}{l}\sigma \theta \\
(0)\end{array}$ & $\begin{array}{c}\rho \\
\left({ }^{\prime \prime}\right)\end{array}$ & $\begin{array}{l}\sigma \rho \\
\left({ }^{\prime \prime}\right)\end{array}$ & $\mathrm{n}$ & Note \\
\hline $23049+0122$ & $\mathrm{~J}$ & 622 & 2016.730 & 320.1 & 0.2 & 4.210 & 0.014 & 1 & \\
\hline $23050+3322$ & $\mathrm{STF}$ & 2974 & 2016.815 & 166.2 & 0.2 & 2.705 & 0.009 & 1 & \\
\hline $23052+6323$ & $\mathrm{KR}$ & 62 & 2016.839 & 324.5 & 0.2 & 5.458 & 0.019 & 1 & \\
\hline $23067-1452$ & HJ & 5384 & 2016.730 & 195.5 & 0.2 & 29.806 & 0.101 & 1 & \\
\hline $23071+6321$ & BHJ & $77 \mathrm{AB}$ & 2016.839 & 319.6 & 0.2 & 25.869 & 0.088 & 1 & \\
\hline $23075+3250$ & $\mathrm{STF}$ & 2978 & 2016.815 & 144.7 & 0.2 & 8.364 & 0.028 & 1 & \\
\hline $23077+0636$ & $\mathrm{STF}$ & $2976 \mathrm{AB}$ & 2016.850 & 261.7 & 0.3 & 7.888 & 0.071 & 2 & \\
\hline $23077+0636$ & $\mathrm{STF}$ & $2976 \mathrm{CB}$ & 2016.850 & 8.7 & 0.2 & 17.830 & 0.055 & 2 & \\
\hline $23080-1304$ & HJ & 3171 & 2016.730 & 40.0 & 0.2 & 23.481 & 0.080 & 1 & \\
\hline $23099-1951$ & HJ & 3173 & 2016.730 & 232.8 & 0.2 & 13.130 & 0.045 & 1 & \\
\hline $23100+1426$ & $\mathrm{STF}$ & 2986 & 2016.865 & 269.9 & 0.1 & 31.485 & 0.047 & 2 & \\
\hline $23101-0804$ & HJ & 3174 & 2016.730 & 19.9 & 0.2 & 5.924 & 0.020 & 1 & \\
\hline $23109+8149$ & $\mathrm{STF}$ & 2996 & 2016.700 & 105.9 & 0.2 & 4.938 & 0.017 & 1 & \\
\hline $23112+2759$ & MLB & 503 & 2016.815 & 157.1 & 0.2 & 7.883 & 0.027 & 1 & \\
\hline $23112+2919$ & $\mathrm{KU}$ & 137 & 2016.815 & 89.0 & 0.2 & 33.761 & 0.115 & 1 & \\
\hline $23120-1156$ & $\mathrm{STF}$ & 2988 & 2016.730 & 97.7 & 0.2 & 3.533 & 0.012 & 1 & \\
\hline $23121+4517$ & $\mathrm{BU}$ & 1528 & 2016.817 & 191.5 & 0.2 & 34.132 & 0.116 & 1 & \\
\hline $23129+1233$ & HJ & 3176 & 2016.849 & 344.4 & 0.1 & 26.891 & 0.018 & 3 & \\
\hline $23154+5937$ & STI & 2964 & 2016.817 & 335.5 & 0.2 & 13.178 & 0.045 & 1 & \\
\hline $23162+5424$ & $\mathrm{~A}$ & 1482 & 2016.817 & 86.8 & 0.2 & 1.299 & 0.004 & 1 & \\
\hline $23169+4238$ & HJ & 1864 & 2016.817 & 205.8 & 0.2 & 22.919 & 0.078 & 1 & \\
\hline $23170+2125$ & $\mathrm{STF}$ & 2997 & 2016.760 & 222.4 & 0.2 & 24.799 & 0.084 & 1 & \\
\hline $23180+1032$ & FOX & 47 & 2016.929 & 280.3 & 0.1 & 4.952 & 0.029 & 3 & \\
\hline $23180+4952$ & $\mathrm{ES}$ & 925 & 2016.817 & 64.0 & 0.2 & 6.218 & 0.021 & 1 & \\
\hline $23185+5409$ & $\mathrm{ES}$ & $695 \mathrm{AB}$ & 2016.817 & 313.1 & 0.2 & 4.072 & 0.014 & 1 & \\
\hline $23185+5409$ & ES & $695 \mathrm{AC}$ & 2016.817 & 301.1 & 0.2 & 24.938 & 0.085 & 1 & \\
\hline $23188+2513$ & $\mathrm{STF}$ & 3000 & 2016.760 & 50.0 & 0.2 & 3.435 & 0.012 & 1 & \\
\hline $23191-1328$ & $\mathrm{STF}$ & $2998 \mathrm{AB}$ & 2016.730 & 352.5 & 0.2 & 12.343 & 0.042 & 1 & \\
\hline $23208+2158$ & $\mathrm{STT}$ & 494 & 2016.826 & 81.5 & 0.2 & 3.308 & 0.011 & 1 & \\
\hline $23239+7704$ & $\mathrm{STF}$ & 3011 & 2016.700 & 332.0 & 0.2 & 6.962 & 0.024 & 1 & \\
\hline $23243+0343$ & $\mathrm{STF}$ & $3009 \mathrm{AB}$ & 2016.826 & 229.6 & 0.2 & 7.132 & 0.024 & 1 & \\
\hline $23244+6917$ & $\mathrm{~A}$ & 789 & 2016.779 & 84.9 & 0.2 & 1.936 & 0.007 & 1 & \\
\hline
\end{tabular}


Table 4-Continued

\begin{tabular}{|c|c|c|c|c|c|c|c|c|c|}
\hline $\begin{array}{l}\text { WDS Desig. } \\
\alpha, \delta(2000)\end{array}$ & & $\begin{array}{l}\text { iscoverer } \\
\text { signation }\end{array}$ & JY & $\begin{array}{c}\theta \\
(0)\end{array}$ & $\begin{array}{l}\sigma \theta \\
(0)\end{array}$ & $\begin{array}{c}\rho \\
\left({ }^{\prime \prime}\right)\end{array}$ & $\begin{array}{l}\sigma \rho \\
\left({ }^{\prime \prime}\right)\end{array}$ & $\mathrm{n}$ & Note \\
\hline $23254-1717$ & HJ & 5398 & 2016.815 & 3.0 & 0.2 & 29.331 & 0.100 & 1 & \\
\hline $23260+2742$ & $\mathrm{HO}$ & $489 \mathrm{AB}$ & 2016.875 & 221.3 & 0.2 & 0.526 & 0.002 & 1 & \\
\hline $23270+5026$ & HJ & 1878 & 2016.779 & 268.4 & 0.2 & 8.921 & 0.030 & 1 & \\
\hline $23276+1638$ & STF & $3012 \mathrm{AB}$ & 2016.826 & 190.2 & 0.2 & 2.857 & 0.010 & 1 & \\
\hline $23276+1638$ & STF & $3013 \mathrm{CD}$ & 2016.826 & 277.2 & 0.2 & 3.239 & 0.011 & 1 & \\
\hline $23277+7406$ & STF & $3017 \mathrm{AB}$ & 2016.700 & 19.1 & 0.2 & 1.208 & 0.004 & 1 & \\
\hline $23280+1024$ & $\mathrm{ROE}$ & 137 & 2016.826 & 61.3 & 0.2 & 8.417 & 0.029 & 1 & \\
\hline $23280+3333$ & STF & 3015 & 2016.839 & 190.0 & 0.2 & 2.955 & 0.010 & 1 & \\
\hline $23292+4042$ & $\mathrm{~A}$ & 1487 & 2016.839 & 157.0 & 0.2 & 1.104 & 0.004 & 1 & \\
\hline $23299-2035$ & HJ & $3196 \mathrm{AB}, \mathrm{C}$ & 2016.818 & 19.4 & 0.2 & 20.449 & 0.070 & 1 & \\
\hline $23309+0929$ & STT & 497 & 2016.826 & 216.4 & 0.2 & 1.405 & 0.005 & 1 & \\
\hline $23314+0317$ & BAL & 2068 & 2016.815 & 74.5 & 0.2 & 7.167 & 0.024 & 1 & \\
\hline $23320+4349$ & STF & 3024 & 2016.839 & 309.1 & 0.2 & 4.839 & 0.016 & 1 & \\
\hline $23341+5947$ & HJ & 1892 & 2016.779 & 94.1 & 0.2 & 6.861 & 0.023 & 1 & \\
\hline $23343-0943$ & $\mathrm{BU}$ & 387 & 2016.815 & 71.5 & 0.2 & 5.877 & 0.020 & 1 & \\
\hline $23350+4731$ & HJ & 1893 & 2016.779 & 248.4 & 0.2 & 5.110 & 0.017 & 1 & \\
\hline $23352+1133$ & HJ & 3203 & 2016.826 & 211.6 & 0.2 & 6.586 & 0.022 & 1 & \\
\hline $23355+0850$ & $\mathrm{BRT}$ & 3372 & 2016.826 & 119.4 & 0.2 & 5.531 & 0.019 & 1 & \\
\hline $23358-1347$ & HJ & 3205 & 2016.815 & 65.0 & 0.2 & 21.261 & 0.072 & 1 & \\
\hline $23362+4944$ & $\mathrm{HU}$ & 791 & 2016.779 & 305.8 & 0.2 & 2.776 & 0.009 & 1 & \\
\hline $23363-0707$ & $\mathrm{BU}$ & $721 \mathrm{AB}$ & 2016.875 & 134.1 & 0.2 & 0.452 & 0.002 & 1 & \\
\hline $23363+2854$ & STF & 3026 & 2016.839 & 274.8 & 0.2 & 3.287 & 0.011 & 1 & \\
\hline $23370+0630$ & $\mathrm{BRT}$ & 2195 & 2016.826 & 170.2 & 0.2 & 4.228 & 0.014 & 1 & \\
\hline $23376+1236$ & HJ & 315 & 2016.826 & 247.6 & 0.2 & 20.551 & 0.070 & 1 & \\
\hline $23388+5637$ & SRW & $12 \mathrm{AB}$ & 2016.779 & 212.2 & 0.2 & 27.645 & 0.094 & 1 & \\
\hline $23388+5637$ & SRW & $12 \mathrm{AC}$ & 2016.779 & 288.1 & 0.2 & 26.035 & 0.089 & 1 & \\
\hline $23402+4949$ & $\mathrm{AG}$ & 427 & 2016.779 & 242.6 & 0.2 & 17.343 & 0.059 & 1 & \\
\hline $23402+7843$ & WFC & 243 & 2016.700 & 228.7 & 0.2 & 5.235 & 0.018 & 1 & \\
\hline $23405+6733$ & $\mathrm{BU}$ & 857 & 2016.779 & 299.4 & 0.2 & 1.207 & 0.004 & 1 & \\
\hline $23407-0023$ & STF & 3030 & 2016.815 & 221.9 & 0.2 & 2.467 & 0.008 & 1 & \\
\hline $23407+4508$ & $\mathrm{BRT}$ & 1162 & 2016.839 & 177.3 & 0.2 & 5.517 & 0.019 & 1 & \\
\hline $23407+6257$ & CLL & $21 \mathrm{AC}$ & 2016.779 & 53.4 & 0.2 & 40.504 & 0.138 & 1 & \\
\hline
\end{tabular}


Table 4-Continued

\begin{tabular}{|c|c|c|c|c|c|c|c|c|c|}
\hline $\begin{array}{l}\text { WDS Desig. } \\
\alpha, \delta(2000)\end{array}$ & & $\begin{array}{l}\text { iscoverer } \\
\text { signation }\end{array}$ & JY & $\begin{array}{c}\theta \\
(0)\end{array}$ & $\begin{array}{l}\sigma \theta \\
(0)\end{array}$ & $\begin{array}{c}\rho \\
\left({ }^{\prime \prime}\right)\end{array}$ & $\begin{array}{l}\sigma \rho \\
\left({ }^{\prime \prime}\right)\end{array}$ & $\mathrm{n}$ & Note \\
\hline $23407+6257$ & SKF & $179 \mathrm{BC}$ & 2016.779 & 43.2 & 0.2 & 32.750 & 0.111 & 1 & \\
\hline $23407+6257$ & STI & $1195 \mathrm{AB}$ & 2016.779 & 88.3 & 0.2 & 10.118 & 0.034 & 1 & \\
\hline $23413+1446$ & STF & 3032 & 2016.826 & 338.8 & 0.2 & 19.440 & 0.066 & 1 & \\
\hline $23420+1523$ & $\mathrm{KU}$ & 140 & 2016.826 & 99.8 & 0.2 & 27.067 & 0.092 & 1 & \\
\hline $23420+2018$ & STT & $503 \mathrm{AB}$ & 2016.826 & 134.1 & 0.2 & 1.033 & 0.004 & 1 & \\
\hline $23428+4727$ & $\mathrm{ES}$ & 548 & 2016.779 & 316.6 & 0.2 & 14.489 & 0.049 & 1 & \\
\hline $23430+4907$ & $\mathrm{BU}$ & 1531 & 2016.779 & 236.4 & 0.2 & 12.844 & 0.044 & 1 & \\
\hline $23435+5805$ & ENG & $88 \mathrm{CE}$ & 2016.779 & 91.1 & 0.2 & 55.655 & 0.189 & 1 & \\
\hline $23438+6904$ & $\mathrm{AG}$ & 294 & 2016.779 & 122.6 & 0.2 & 17.034 & 0.058 & 1 & \\
\hline $23439+0715$ & STF & 3033 & 2016.826 & 182.8 & 0.2 & 3.058 & 0.010 & 1 & \\
\hline $23439+6633$ & HDS & 3370 & 2016.779 & 1.5 & 0.2 & 15.155 & 0.052 & 1 & \\
\hline $23455+1223$ & $\mathrm{AG}$ & 428 & 2016.826 & 86.3 & 0.2 & 4.660 & 0.016 & 1 & \\
\hline $23459+3039$ & MLB & 673 & 2016.839 & 291.4 & 0.2 & 6.571 & 0.022 & 1 & \\
\hline $23460-1841$ & H 2 & 24 & 2016.815 & 135.2 & 0.2 & 7.011 & 0.024 & 1 & \\
\hline $23461+6028$ & STF & $3037 \mathrm{AB}$ & 2016.779 & 212.4 & 0.2 & 2.545 & 0.009 & 1 & \\
\hline $23461+6028$ & STF & $3037 \mathrm{AC}$ & 2016.779 & 189.6 & 0.2 & 29.521 & 0.100 & 1 & \\
\hline $23461+6028$ & STF & $3037 \mathrm{AD}$ & 2016.779 & 233.4 & 0.2 & 52.902 & 0.180 & 1 & \\
\hline $23461+6028$ & STF & $3037 \mathrm{BC}$ & 2016.779 & 187.6 & 0.2 & 27.204 & 0.092 & 1 & \\
\hline $23465+1705$ & EGB & 8 & 2016.826 & 86.0 & 0.2 & 1.053 & 0.004 & 1 & \\
\hline $23468+4640$ & ES & 550 & 2016.779 & 167.7 & 0.2 & 16.876 & 0.057 & 1 & \\
\hline $23469+4340$ & $\mathrm{ES}$ & 1475 & 2016.839 & 303.7 & 0.2 & 5.593 & 0.019 & 1 & \\
\hline $23470+0515$ & BAR & 19 & 2016.826 & 2.8 & 0.2 & 1.113 & 0.004 & 1 & \\
\hline $23471-1921$ & DON & 1053 & 2016.815 & 275.6 & 0.2 & 7.108 & 0.024 & 1 & \\
\hline $23479+1703$ & STF & $3041 \mathrm{AB}$ & 2016.826 & 358.1 & 0.2 & 56.362 & 0.192 & 1 & \\
\hline $23479+1703$ & STF & $3041 \mathrm{~A}, \mathrm{BC}$ & 2016.826 & 358.2 & 0.2 & 58.297 & 0.198 & 1 & \\
\hline $23479+1703$ & $\mathrm{STF}$ & $3041 \mathrm{AC}$ & 2016.826 & 358.1 & 0.2 & 59.954 & 0.204 & 1 & \\
\hline $23479+1703$ & STF & $3041 \mathrm{BC}$ & 2016.826 & 356.5 & 0.2 & 3.504 & 0.012 & 1 & \\
\hline $23483+8532$ & WFC & $244 \mathrm{AB}$ & 2016.700 & 6.4 & 0.2 & 4.730 & 0.016 & 1 & \\
\hline $23487+6453$ & STT & $507 \mathrm{AC}$ & 2016.785 & 349.9 & 0.2 & 50.754 & 0.173 & 1 & \\
\hline $23505+4325$ & STT & 509 & 2016.779 & 105.3 & 0.2 & 5.498 & 0.019 & 1 & \\
\hline $23509-1249$ & JKS & 25 & 2016.818 & 23.6 & 0.2 & 28.193 & 0.096 & 1 & \\
\hline $23512-1903$ & HJ & 3219 & 2016.818 & 344.5 & 0.2 & 10.599 & 0.036 & 1 & \\
\hline
\end{tabular}


Table 4-Continued

\begin{tabular}{|c|c|c|c|c|c|c|c|c|c|}
\hline $\begin{array}{l}\text { WDS Desig. } \\
\alpha, \delta(2000)\end{array}$ & & $\begin{array}{l}\text { iscoverer } \\
\text { signation }\end{array}$ & JY & $\begin{array}{l}\theta \\
(0)\end{array}$ & $\begin{array}{l}\sigma \theta \\
(0)\end{array}$ & $\begin{array}{c}\rho \\
\left({ }^{\prime \prime}\right)\end{array}$ & $\begin{array}{l}\sigma \rho \\
\left({ }^{\prime \prime}\right)\end{array}$ & $\mathrm{n}$ & Note \\
\hline $23516+0841$ & CHE & 506 & 2016.818 & 79.3 & 0.2 & 8.954 & 0.030 & 1 & \\
\hline $23522+4331$ & $\mathrm{BU}$ & $728 \mathrm{AB}$ & 2016.779 & 10.0 & 0.2 & 1.250 & 0.004 & 1 & \\
\hline $23525+2853$ & $\mathrm{~J}$ & 213 & 2016.818 & 166.7 & 0.2 & 5.421 & 0.018 & 1 & \\
\hline $23527+2920$ & $\mathrm{AG}$ & 429 & 2016.818 & 268.8 & 0.2 & 6.362 & 0.022 & 1 & \\
\hline $23528+3841$ & STF & $3043 \mathrm{AB}, \mathrm{C}$ & 2016.817 & 250.0 & 0.2 & 15.765 & 0.054 & 1 & \\
\hline $23531-0319$ & $\mathrm{~J}$ & 1429 & 2016.818 & 304.4 & 0.2 & 9.164 & 0.031 & 1 & \\
\hline $23532+6157$ & ES & 1932 & 2016.785 & 340.6 & 0.2 & 6.642 & 0.023 & 1 & \\
\hline $23535+5136$ & $\mathrm{ACA}$ & 17 & 2016.785 & 87.5 & 0.2 & 56.759 & 0.193 & 1 & \\
\hline $23555+5605$ & STI & 3056 & 2016.785 & 158.2 & 0.2 & 9.414 & 0.032 & 1 & \\
\hline $23557+3830$ & $\mathrm{AG}$ & 296 & 2016.818 & 53.8 & 0.2 & 5.632 & 0.019 & 1 & \\
\hline $23566-1445$ & HJ & $3227 \mathrm{AB}$ & 2016.818 & 300.6 & 0.2 & 19.296 & 0.066 & 1 & \\
\hline $23573+6102$ & ARG & $99 \mathrm{CD}$ & 2016.785 & 317.4 & 0.2 & 4.647 & 0.016 & 1 & \\
\hline $23576+4804$ & A & 799 & 2016.779 & 191.4 & 0.2 & 2.092 & 0.007 & 1 & \\
\hline $23579+5723$ & STF & $3047 \mathrm{AB}$ & 2016.785 & 72.0 & 0.2 & 1.167 & 0.004 & 1 & \\
\hline $23581+5539$ & STI & 3070 & 2016.785 & 211.2 & 0.2 & 4.573 & 0.016 & 1 & \\
\hline $23590+5315$ & HLD & $59 \mathrm{AB}$ & 2016.785 & 11.3 & 0.2 & 1.206 & 0.004 & 1 & \\
\hline $23591+5658$ & ES & 38 & 2016.785 & 237.0 & 0.2 & 24.528 & 0.083 & 1 & \\
\hline $23592+7448$ & $\mathrm{BU}$ & 1154 & 2016.700 & 332.7 & 0.2 & 1.278 & 0.004 & 1 & \\
\hline $23593+5352$ & ES & 2736 & 2016.785 & 107.0 & 0.2 & 19.348 & 0.066 & 1 & \\
\hline $23596-0748$ & $\mathrm{BU}$ & 731 & 2016.818 & 297.4 & 0.2 & 1.692 & 0.006 & 1 & \\
\hline
\end{tabular}

A : This would have been published in an earlier paper in this series, but given the large change in position this measure was not published until its confirmation.

B : First measure of this pair. See Tables $2 \& 3$.

C : Confirming observation. 
Table 5. ICCD Measurements of Double Stars with Orbit \& Linear Residuals

\begin{tabular}{|c|c|c|c|c|c|c|c|c|c|c|c|c|}
\hline \multirow{2}{*}{$\begin{array}{c}\text { WDS Desig. } \\
\alpha, \delta(2000) \\
00014+3937\end{array}$} & \multicolumn{2}{|c|}{$\begin{array}{c}\text { Discoverer } \\
\text { Designation }\end{array}$} & \multirow{2}{*}{$\begin{array}{c}\text { JY } \\
2016.850\end{array}$} & \multirow{2}{*}{$\begin{array}{c}\begin{array}{c}\theta \\
(\circ)\end{array} \\
166.6\end{array}$} & \multirow{2}{*}{$\begin{array}{l}\sigma \theta \\
(0) \\
0.2\end{array}$} & \multirow{2}{*}{$\begin{array}{c}\begin{array}{c}\rho \\
\left({ }^{\prime \prime}\right)\end{array} \\
1.340\end{array}$} & \multirow{2}{*}{$\begin{array}{l}\begin{array}{c}\sigma \rho \\
\left({ }^{\prime \prime}\right)\end{array} \\
0.005\end{array}$} & \multirow[t]{2}{*}{$\mathrm{n}$} & \multirow{2}{*}{$\begin{array}{r}\begin{array}{c}\mathrm{O}-\mathrm{C}_{\theta} \\
(0)\end{array} \\
1.1\end{array}$} & \multirow{2}{*}{$\begin{array}{l}\begin{array}{c}\mathrm{O}-\mathrm{C}_{\rho} \\
\left({ }^{\prime \prime}\right)\end{array} \\
0.032\end{array}$} & Reference & Note \\
\hline & HLD & 60 & & & & & & & & & Hartkopf \& Mason 2011a & A \\
\hline $00032+4508$ & HJ & 1927 & 2016.853 & 73.1 & 0.2 & 9.590 & 0.033 & 1 & 0.4 & -0.028 & Hartkopf \& Mason 2011b & \\
\hline $00057+4549$ & STT & $547 \mathrm{AB}$ & 2016.853 & 189.2 & 0.2 & 5.984 & 0.020 & 1 & $\begin{array}{r}-0.2 \\
0.3\end{array}$ & $\begin{array}{r}0.084 \\
-0.006\end{array}$ & $\begin{array}{l}\text { Popovic \& Pavlovic } 1996 \\
\text { Kiyaeva et al. } 2001\end{array}$ & A \\
\hline $00162+7657$ & STF & 13 & 2016.785 & 48.2 & 0.2 & 0.958 & 0.003 & 1 & $\begin{array}{r}0.3 \\
-0.4\end{array}$ & $\begin{array}{l}0.050 \\
0.002\end{array}$ & $\begin{array}{l}\text { Olevic \& Jovanovic } 2001 \\
\text { Table } 6\end{array}$ & \\
\hline $00209+3259$ & $\mathrm{AC}$ & 1 & 2016.850 & 289.9 & 0.2 & 1.846 & 0.006 & 1 & 1.0 & 0.004 & Zirm 2015 & \\
\hline $00272+4959$ & STF & $30 \mathrm{AB}$ & 2016.850 & 315.8 & 0.2 & 13.249 & 0.045 & 1 & 0.9 & -0.054 & Hartkopf \& Mason 2011b & \\
\hline $00287+3718$ & A & $1504 \mathrm{AB}$ & 2016.850 & 49.3 & 0.2 & 0.581 & 0.002 & 1 & 4.0 & -0.015 & Zirm 2014 & A \\
\hline $00360+2959$ & $\mathrm{STF}$ & $42 \mathrm{AB}$ & 2016.815 & 20.8 & 0.2 & 6.265 & 0.021 & 1 & 0.5 & -0.050 & Kiselev et al. 2009 & \\
\hline $00360+2959$ & STF & $42 \mathrm{AC}$ & 2016.815 & 289.6 & 0.2 & 36.681 & 0.125 & 1 & 0.6 & 0.039 & Hartkopf \& Mason 2011b & \\
\hline $00384+4059$ & $\mathrm{STF}$ & 44 & 2016.779 & 274.2 & 0.2 & 12.967 & 0.044 & 1 & 0.2 & 0.199 & Hartkopf \& Mason 2011b & B \\
\hline $00464+3057$ & STFA & $1 \mathrm{AB}$ & 2016.815 & 46.5 & 0.2 & 47.569 & 0.162 & 1 & 0.3 & 0.244 & Hartkopf \& Mason 2011b & \\
\hline $00521+1036$ & STF & 67 & 2016.839 & 349.2 & 0.2 & 2.313 & 0.008 & 1 & -0.1 & 0.026 & Hartkopf \& Mason 2011a & A \\
\hline $00594+0047$ & STF & $80 \mathrm{AB}$ & 2016.849 & 338.9 & 0.2 & 29.801 & 0.025 & 2 & 0.3 & 0.145 & Hartkopf \& Mason 2011b & \\
\hline $01032+2006$ & LDS & 873 & 2004.852 & 51.2 & 0.2 & 2.556 & 0.009 & 2 & 0.1 & -0.033 & Rica et al. 2012 & $\mathrm{~A}, \mathrm{C}$ \\
\hline $01048-0528$ & STF & $86 \mathrm{AB}$ & 2016.776 & 137.3 & 0.3 & 16.847 & 0.014 & 2 & 0.2 & 0.061 & Hartkopf \& Mason 2011b & \\
\hline $01198-0031$ & $\mathrm{STF}$ & $113 \mathrm{~A}, \mathrm{BC}$ & 2016.849 & 21.4 & 0.2 & 1.623 & 0.008 & 2 & 0.3 & -0.015 & Zirm 2015 & \\
\hline $01200-1549$ & HJ & 2036 & 2016.818 & 338.1 & 0.2 & 2.398 & 0.008 & 1 & 0.4 & 0.028 & Olevic et al. 2003 & A \\
\hline $01207+4620$ & STF & $112 \mathrm{AB}$ & 2016.850 & 337.4 & 0.2 & 19.042 & 0.065 & 1 & 0.8 & 0.101 & Hartkopf \& Mason 2011b & \\
\hline $01211+6439$ & $\mathrm{~S}$ & 397 & 2016.850 & 342.1 & 0.2 & 57.396 & 0.195 & 1 & 0.7 & 0.098 & Hartkopf \& Mason 2011b & \\
\hline $01399+1515$ & STF & $142 \mathrm{AB}$ & 2016.818 & 68.1 & 0.2 & 23.311 & 0.079 & 1 & 0.2 & 0.249 & Hartkopf \& Mason 2011b & B \\
\hline $01456-2503$ & HJ & $3461 \mathrm{AB}$ & 2002.900 & 23.1 & 0.2 & 4.898 & 0.017 & 1 & -1.5 & -0.050 & Mason \& Hartkopf 2014 & $\mathrm{~A}, \mathrm{C}$ \\
\hline $01467+3310$ & STF & $158 \mathrm{AB}$ & 2016.883 & 271.5 & 0.2 & 2.230 & 0.008 & 1 & -1.5 & 0.213 & Hartkopf \& Mason 2011a & A \\
\hline $01488-0125$ & STF & $171 \mathrm{AB}$ & 2016.891 & 165.4 & 0.2 & 34.513 & 0.117 & 1 & 0.7 & 0.197 & Hartkopf \& Mason 2011b & \\
\hline $01493+4754$ & $\mathrm{STF}$ & $162 \mathrm{AB}$ & 2016.967 & 199.0 & 0.0 & 1.898 & 0.034 & 2 & $\begin{array}{l}0.3 \\
0.3\end{array}$ & $\begin{array}{l}-0.059 \\
-0.059\end{array}$ & $\begin{array}{l}\text { Zirm \& Rica } 2014 \\
\text { Genet et al. } 2015\end{array}$ & B \\
\hline $01510+2107$ & STF & $175 \mathrm{AB}$ & 2016.891 & 0.2 & 0.2 & 28.381 & 0.096 & 1 & 0.8 & 0.056 & Hartkopf \& Mason 2011b & \\
\hline $02216+2338$ & STF & 254 & 2016.926 & 16.5 & 0.1 & 12.111 & 0.013 & 2 & 0.4 & 0.003 & Hartkopf \& Mason 2011b & \\
\hline $02231+7021$ & MLR & $377 \mathrm{AB}$ & 2004.885 & 144.4 & 0.2 & 0.655 & 0.002 & 2 & -1.5 & 0.084 & Pavlovic \& Todorovic 2005 & $\mathrm{C}$ \\
\hline $02407+6117$ & STF & $284 \mathrm{AB}$ & 2016.836 & 191.2 & 0.3 & 6.906 & 0.013 & 2 & 1.0 & -0.023 & Hartkopf \& Mason 2011b & \\
\hline $02475+1922$ & STF & $305 \mathrm{AB}$ & 2016.856 & 307.6 & 0.0 & 3.645 & 0.004 & 3 & 0.6 & 0.043 & Mason \& Hartkopf 2014 & A \\
\hline $02558+3429$ & STF & $325 \mathrm{AB}$ & 2016.846 & 147.4 & 0.0 & 23.261 & 0.014 & 2 & 1.1 & 0.094 & Hartkopf \& Mason 2011b & \\
\hline $02563+7253$ & STF & $312 \mathrm{AB}$ & 2016.875 & 48.8 & 0.2 & 1.777 & 0.006 & 1 & 2.5 & 0.017 & Cvetkovic \& Novakovic 2006 & \\
\hline $02592+2120$ & STF & $333 \mathrm{AB}$ & 2016.888 & 210.6 & 0.0 & 1.371 & 0.002 & 3 & 0.8 & 0.038 & Rica 2012 & A \\
\hline $03121-2859$ & $\mathrm{HJ}$ & 3555 & 2002.900 & 299.1 & 0.2 & 4.880 & 0.017 & 1 & -0.1 & -0.127 & Söderhjelm 1999 & $\mathrm{~A}, \mathrm{C}$ \\
\hline $03127+7133$ & STT & $50 \mathrm{AB}$ & 2016.875 & 146.2 & 0.2 & 1.026 & 0.003 & 1 & 3.0 & 0.132 & Scardia et al. 2012 & $\mathrm{~A}$ \\
\hline $03140+0044$ & STF & 367 & 2016.935 & 129.8 & 0.2 & 1.232 & 0.004 & 1 & -0.2 & -0.013 & Riddle et al. 2015 & A \\
\hline $03196+6714$ & $\mathrm{HU}$ & 1056 & 2016.881 & 80.6 & 0.2 & 1.035 & 0.004 & 1 & 0.8 & -0.027 & Zirm 2015 & A \\
\hline $03207+4641$ & BU & 1294 & 2016.990 & 240.1 & 0.2 & 8.714 & 0.030 & 1 & 1.1 & 0.033 & Hartkopf \& Mason 2011b & \\
\hline $03217+0845$ & STF & 380 & 2016.935 & 6.6 & 0.2 & 0.923 & 0.003 & 1 & 4.2 & 0.041 & Popovic \& Pavlovic 1996 & A \\
\hline $03480+6840$ & WNO & $16 \mathrm{AD}$ & 2016.051 & 352.4 & 0.2 & 30.446 & 0.104 & 1 & 1.1 & 0.093 & Hartkopf \& Mason 2011b & \\
\hline $04422+3731$ & STF & 577 & 2016.990 & 331.8 & 0.2 & 0.620 & 0.002 & 1 & 4.2 & -0.062 & Riddle et al. 2015 & A \\
\hline $04465+7128$ & HJ & 2235 & 2016.853 & 156.0 & 0.2 & 40.587 & 0.138 & 1 & 0.9 & 0.204 & Hartkopf \& Mason 2011b & \\
\hline $05013+5015$ & STF & 619 & 2016.037 & 161.2 & 0.2 & 4.126 & 0.014 & 1 & 1.9 & 0.034 & Kiselev et al. 2009 & $\mathrm{D}$ \\
\hline $05047+7404$ & STT & 89 & 2002.996 & 304.0 & 0.2 & 0.329 & 0.001 & 1 & 0.6 & -0.018 & Alzner 1998 & $\mathrm{~A}, \mathrm{C}$ \\
\hline $05364+2200$ & STF & 742 & 2016.990 & 275.4 & 0.2 & 4.152 & 0.014 & 1 & -0.3 & 0.003 & Hopmann 1973 & \\
\hline $05446+6320$ & STI & 579 & 2016.051 & 121.2 & 0.2 & 8.389 & 0.029 & 1 & -1.6 & 0.455 & Hurowitz et al. 2013 & B \\
\hline $06482+5542$ & STF & $958 \mathrm{AB}$ & 2016.212 & 76.2 & 0.2 & 4.159 & 0.014 & 1 & -0.7 & -0.367 & Kiselev et al. 2009 & \\
\hline $06487+0737$ & A & $2731 \mathrm{AB}$ & 2005.170 & 64.4 & 0.2 & 1.195 & 0.004 & 1 & $\begin{array}{r}-0.3 \\
0.3\end{array}$ & $\begin{array}{l}-0.297 \\
-0.088\end{array}$ & $\begin{array}{l}\text { Kiselev et al. } 2009 \\
\text { Prieur et al. } 2012\end{array}$ & $\mathrm{C}$ \\
\hline $07201+2159$ & STF & 1066 & 2002.172 & 224.1 & 0.2 & 5.889 & 0.020 & 2 & -1.4 & 0.160 & Hopmann 1960 & $\mathrm{~A}, \mathrm{C}$ \\
\hline $08122+1739$ & STF & $1196 \mathrm{AB}, \mathrm{C}$ & 2004.906 & 72.6 & 0.2 & 6.013 & 0.020 & 2 & 1.9 & 0.105 & Heintz 1996 & A,C \\
\hline $08122+1739$ & $\mathrm{STF}$ & $1196 \mathrm{AB}, \mathrm{C}$ & 2005.287 & 71.8 & 0.2 & 6.262 & 0.021 & 1 & 1.4 & 0.354 & Heintz 1996 & $\mathrm{C}$ \\
\hline $08122+1739$ & $\mathrm{STF}$ & $1196 \mathrm{AB}, \mathrm{C}$ & 2016.832 & 64.2 & 0.2 & 5.959 & 0.020 & 1 & -1.2 & 0.030 & Heintz 1996 & \\
\hline $08269+3212$ & $\mathrm{HU}$ & $714 \mathrm{Ba}, \mathrm{Bb}$ & 2004.160 & 309.4 & 0.2 & 0.723 & 0.002 & 1 & $\begin{array}{l}-6.3 \\
-0.0\end{array}$ & $\begin{array}{l}0.146 \\
0.003\end{array}$ & $\begin{array}{l}\text { Baize } 1993 \\
\text { Table } 6\end{array}$ & $\mathrm{C}$ \\
\hline $08369+2315$ & $\mathrm{AG}$ & 154 & 2016.832 & 1.3 & 0.2 & 2.678 & 0.009 & 1 & 0.4 & 0.002 & Hartkopf \& Mason 2011c & \\
\hline $08568-1726$ & ARG & $72 \mathrm{AB}$ & 2016.996 & 182.6 & 0.2 & 4.053 & 0.014 & 1 & 0.6 & 0.029 & Hartkopf \& Mason 2011b & \\
\hline $09013+1516$ & STF & $1300 \mathrm{AB}$ & 2016.163 & 177.5 & 0.2 & 5.029 & 0.017 & 1 & -1.4 & -0.005 & Zirm 2008 & \\
\hline $09079-0708$ & $\mathrm{STF}$ & $1316 \mathrm{AC}$ & 2016.172 & 279.5 & 0.3 & 7.949 & 0.010 & 2 & -0.1 & 0.031 & Hartkopf \& Mason 2011b & \\
\hline $09079-0708$ & STF & $1316 \mathrm{BC}$ & 2016.204 & 297.1 & 0.2 & 14.218 & 0.048 & 1 & 2.2 & 0.104 & Friedman et al. 2012 & \\
\hline $09144+5241$ & $\mathrm{STF}$ & $1321 \mathrm{AB}$ & 2016.213 & 98.1 & 0.2 & 17.102 & 0.058 & 1 & -0.1 & 0.255 & Chang 1972 & A \\
\hline $09157-0114$ & STF & $1329 \mathrm{AB}$ & 2016.196 & 265.4 & 0.1 & 8.301 & 0.036 & 2 & -0.0 & 0.046 & Hartkopf \& Mason 2011b & \\
\hline $10160+1200$ & HJ & $156 \mathrm{BC}$ & 2016.163 & 355.3 & 0.2 & 29.664 & 0.101 & 1 & 0.2 & -0.162 & Hurowitz et al. 2014 & \\
\hline $10200+1950$ & STF & $1424 \mathrm{AB}$ & 2016.213 & 125.8 & 0.2 & 4.898 & 0.017 & 1 & -1.4 & 0.163 & Romanenko \& Kiselev 2014 & A \\
\hline & & & & & & & & & -0.6 & 0.183 & Romanenko \& Kiselev 2014 & \\
\hline $11268+0301$ & STF & $1540 \mathrm{AB}$ & 2016.163 & 149.6 & 0.2 & 28.086 & 0.095 & 1 & 2.8 & -0.532 & Hopmann 1960 & \\
\hline
\end{tabular}


Table 5-Continued

\begin{tabular}{|c|c|c|c|c|c|c|c|c|c|c|c|c|}
\hline \multirow{2}{*}{$\begin{array}{c}\text { WDS Desig. } \\
\alpha, \delta(2000) \\
11279+0251\end{array}$} & \multicolumn{2}{|c|}{$\begin{array}{c}\text { Discoverer } \\
\text { Designation }\end{array}$} & \multirow{2}{*}{$\begin{array}{c}\text { JY } \\
2016.169\end{array}$} & \multirow{2}{*}{$\begin{array}{c}\theta \\
(\circ) \\
1816\end{array}$} & \multirow{2}{*}{$\begin{array}{l}\sigma \theta \\
(0) \\
0.2\end{array}$} & \multirow{2}{*}{$\begin{array}{c}\begin{array}{c}\rho \\
\left({ }^{\prime \prime}\right)\end{array} \\
88.411\end{array}$} & \multirow{2}{*}{$\begin{array}{l}\begin{array}{l}\sigma \rho \\
\left({ }^{\prime \prime}\right)\end{array} \\
0.301\end{array}$} & \multirow[t]{2}{*}{$\mathrm{n}$} & \multirow{2}{*}{$\begin{array}{c}\mathrm{O}-\mathrm{C}_{\theta} \\
(0) \\
-0.2\end{array}$} & $\underset{\left({ }^{\prime \prime}\right)}{\mathrm{O}-\mathrm{C}_{\rho}}$ & Reference & Note \\
\hline & STFA & $19 \mathrm{AB}$ & & & & & & & & -0.339 & Hartkopf \& Mason 2011b & \\
\hline $12078+3110$ & SEI & 527 & 2016.185 & 6.7 & 0.2 & 42.253 & 0.144 & 1 & 0.2 & -0.098 & Hartkopf \& Mason 2011b & \\
\hline $13379+4808$ & $\mathrm{ES}$ & $608 \mathrm{AB}$ & 2002.383 & 323.5 & 0.2 & 1.942 & 0.007 & 2 & 3.8 & 0.009 & Seymour et al. 2002 & $\mathrm{~A}, \mathrm{C}$ \\
\hline $13379+4808$ & $\mathrm{ES}$ & $608 \mathrm{AB}$ & 2005.403 & 322.1 & 0.2 & 1.752 & 0.006 & 1 & -0.2 & -0.115 & Seymour et al. 2002 & $\mathrm{C}$ \\
\hline $13550-0804$ & $\mathrm{STF}$ & $1788 \mathrm{AB}$ & 2005.356 & 98.7 & 0.2 & 3.635 & 0.012 & 1 & 0.1 & 0.104 & Hopmann 1970 & $\mathrm{~A}, \mathrm{C}$ \\
\hline $14203+4830$ & STF & 1834 & 2016.412 & 105.4 & 0.2 & 1.656 & 0.006 & 1 & 3.0 & 0.050 & Hartkopf \& Mason 2015 & $\mathrm{~A}$ \\
\hline $14410+5757$ & $\mathrm{STF}$ & $1872 \mathrm{AB}$ & 2016.467 & 50.8 & 0.2 & 7.602 & 0.026 & 1 & 0.6 & 0.033 & Kiyaeva et al. 2010 & \\
\hline $14463+0939$ & STF & $1879 \mathrm{AB}$ & 2016.412 & 82.6 & 0.2 & 1.720 & 0.006 & 1 & -0.2 & 0.000 & Mason et al. 1999 & \\
\hline $14464-0723$ & STF & $1876 \mathrm{AB}$ & 2016.412 & 114.9 & 0.2 & 1.237 & 0.004 & 1 & 1.5 & -0.006 & Seymour et al. 2002 & \\
\hline $14497+4843$ & STF & 1890 & 2016.467 & 46.8 & 0.2 & 2.659 & 0.009 & 1 & 1.0 & 0.077 & Hartkopf \& Mason 2011b & B \\
\hline $14534+1542$ & STT & 288 & 2016.412 & 160.5 & 0.2 & 1.006 & 0.003 & 1 & 2.8 & 0.013 & Heintz 1998 & $\mathrm{~A}$ \\
\hline $15038+4739$ & STF & 1909 & 2016.467 & 71.4 & 0.2 & 0.762 & 0.003 & 1 & -0.2 & -0.003 & Zirm 2011 & \\
\hline $15382+3615$ & $\mathrm{STF}$ & $1964 \mathrm{CD}$ & 2016.451 & 21.8 & 0.2 & 1.546 & 0.005 & 1 & 2.0 & 0.038 & Drummond et al. 1995 & \\
\hline $16060+1319$ & STF & $2007 \mathrm{AB}$ & 2016.544 & 323.7 & 0.2 & 38.992 & 0.133 & 1 & 1.8 & 0.615 & Hartkopf \& Mason 2011b & $\mathrm{B}, \mathrm{C}$ \\
\hline $16081+1703$ & STF & $2010 \mathrm{AB}$ & 2016.544 & 15.4 & 0.2 & 27.280 & 0.093 & 1 & 1.8 & 0.244 & Hartkopf \& Mason 2011b & \\
\hline $16147+3352$ & STF & $2032 \mathrm{AB}$ & 2016.544 & 240.8 & 0.2 & 7.369 & 0.025 & 1 & 2.3 & 0.161 & Raghavan et al. 2009 & A \\
\hline $16160+0721$ & STF & $2026 \mathrm{AB}$ & 2016.544 & $\begin{array}{r}240.9 \\
18.9\end{array}$ & 0.2 & 3.573 & 0.012 & 1 & 2.0 & 0.049 & Scardia et al. 2011 & $\mathrm{~A}$ \\
\hline $16289+1825$ & STF & $2052 \mathrm{AB}$ & 2016.544 & 121.0 & 0.2 & 2.422 & 0.008 & 1 & 2.2 & 0.024 & Scardia et al. 2015c & \\
\hline $16439+4329$ & $\mathrm{D}$ & 15 & 2002.467 & 92.9 & 0.2 & 0.425 & 0.001 & 4 & -3.5 & -0.014 & Alzner 2007 & $\mathrm{~A}, \mathrm{C}$ \\
\hline $17010+6807$ & MLR & $199 \mathrm{AB}$ & 2016.481 & 76.5 & 0.1 & 19.245 & 0.014 & 2 & 1.7 & -0.061 & Hartkopf \& Mason 2011b & \\
\hline $17053+5428$ & STF & $2130 \mathrm{AB}$ & 2016.467 & 3.2 & 0.2 & 2.550 & 0.009 & 1 & 1.7 & 0.026 & Prieur et al. 2012 & A \\
\hline $17146+1423$ & STF & $2140 \mathrm{AB}$ & 2005.663 & 104.2 & 0.2 & 4.806 & 0.016 & 4 & 0.3 & 0.160 & Baize 1978 & $\mathrm{~A}, \mathrm{C}$ \\
\hline $17146+1423$ & $\mathrm{STF}$ & $2140 \mathrm{AB}$ & 2016.642 & 103.5 & 0.2 & 4.832 & 0.016 & 1 & 0.7 & 0.190 & Baize 1978 & \\
\hline $17419+7209$ & STF & $2241 \mathrm{AB}$ & 2016.653 & 16.2 & 0.2 & 30.122 & 0.102 & 1 & -0.8 & 0.544 & Kiselev et al. 2009 & A \\
\hline $17520+1520$ & STT & $338 \mathrm{AB}$ & 2016.544 & 167.7 & 0.2 & 0.816 & 0.003 & 1 & 4.1 & -0.013 & Prieur et al. 2012 & $\mathrm{~A}$ \\
\hline $18002+8000$ & STF & $2308 \mathrm{AB}$ & 2016.653 & 231.8 & 0.2 & 18.736 & 0.064 & 1 & $\begin{array}{l}-0.1 \\
-0.1\end{array}$ & $\begin{array}{r}-0.071 \\
0.006\end{array}$ & $\begin{array}{l}\text { Kiselev \& Romanenko } 1996 \\
\text { Hartkopf \& Mason 2011b }\end{array}$ & \\
\hline $18032+2522$ & STF & $2268 \mathrm{AC}$ & 2016.645 & 201.9 & 0.2 & 24.521 & 0.083 & 1 & -0.5 & 0.440 & Hartkopf \& Mason 2011b & B \\
\hline $18044+0329$ & STF & $2266 \mathrm{AC}$ & 2016.552 & 203.0 & 0.2 & 50.802 & 0.173 & 1 & 2.0 & 0.088 & Hartkopf \& Mason 2011b & \\
\hline $18055+0230$ & STF & $2272 \mathrm{AB}$ & 2016.552 & 127.2 & 0.2 & 6.448 & 0.022 & 1 & 2.6 & 0.048 & Eggenberger et al. 2008 & \\
\hline $18101+1629$ & STF & 2289 & 2016.552 & 220.6 & 0.2 & 1.215 & 0.004 & 1 & 5.0 & -0.026 & Hopmann 1964 & A \\
\hline $18146+0011$ & STF & 2294 & 2016.642 & 93.3 & 0.2 & 1.362 & 0.005 & 1 & 0.1 & 0.034 & Scardia et al. 2015b & $\mathrm{A}$ \\
\hline $18312+1311$ & STF & 2330 & 2016.664 & 165.4 & 0.2 & 16.386 & 0.056 & 1 & -0.1 & 0.123 & Hartkopf \& Mason 2011b & \\
\hline $18443+3940$ & STF & $2382 \mathrm{AB}$ & 2016.689 & 345.9 & 0.2 & 2.297 & 0.008 & 1 & 0.3 & -0.035 & Mason et al. 2004 & A \\
\hline & & & & & & & & & 0.4 & 0.047 & Novakovic \& Todorovic 2006 & \\
\hline $18443+3940$ & STF & $2383 \mathrm{CD}$ & 2016.689 & 76.3 & 0.2 & 2.404 & 0.008 & 1 & 0.8 & 0.014 & Docobo \& Costa 1984 & \\
\hline $18489+1615$ & STF & $2400 \mathrm{~A}, \mathrm{BC}$ & 2016.648 & 160.8 & 0.2 & 11.034 & 0.038 & 1 & -0.2 & 0.229 & Cvetkovic et al. 2016 & B \\
\hline $19022+0845$ & STF & $2436 \mathrm{AB}$ & 2016.642 & 314.9 & 0.2 & 30.296 & 0.103 & 1 & -0.1 & 0.212 & Hartkopf \& Mason 2011b & \\
\hline $19027-0043$ & STF & $2434 \mathrm{~A}, \mathrm{BC}$ & 2016.642 & 88.8 & 0.2 & 27.726 & 0.094 & 1 & -0.3 & 0.112 & Hartkopf \& Mason 2011b & \\
\hline $19121+4951$ & $\mathrm{STF}$ & $2486 \mathrm{AB}$ & 2005.720 & 205.7 & 0.2 & 7.300 & 0.025 & 1 & 0.0 & -0.109 & Hale 1994 & A.C \\
\hline $19121+4951$ & STF & $2486 \mathrm{AB}$ & 2016.702 & 204.2 & 0.2 & 7.216 & 0.025 & 1 & 0.2 & 0.004 & Hale 1994 & \\
\hline $19169+6312$ & STF & 2509 & 2016.700 & 328.2 & 0.2 & 1.869 & 0.006 & 1 & -0.3 & 0.028 & Zirm 2014 & A \\
\hline $19266+2530$ & STF & $2524 \mathrm{AB}$ & 2016.645 & 81.9 & 0.2 & 5.427 & 0.018 & 1 & -0.4 & 0.014 & Hartkopf \& Mason 2011b & \\
\hline $19266+2719$ & STF & $2525 \mathrm{AB}$ & 2016.645 & 289.2 & 0.2 & 2.223 & 0.008 & 1 & -0.2 & 0.045 & Scardia et al. 2015a & A \\
\hline $19282-1209$ & SCJ & 22 & 2016.653 & 288.9 & 0.2 & 1.104 & 0.004 & 1 & -3.1 & 0.016 & Docobo \& Ling 2009 & \\
\hline & & & & & & & & & -0.1 & -0.002 & Table 6 & \\
\hline $19368-1027$ & STF & 2541 & 2016.656 & 326.1 & 0.2 & 5.500 & 0.019 & 1 & -0.3 & -0.197 & Hartkopf \& Mason 2011b & B \\
\hline $20014+1045$ & $\mathrm{STF}$ & $2613 \mathrm{AB}$ & 2016.664 & 355.1 & 0.2 & 3.581 & 0.012 & 1 & 3.0 & -0.564 & Hopmann 1973 & $\mathrm{~A}$ \\
\hline $20099+2055$ & STF & $2637 \mathrm{AC}$ & 2016.814 & 221.5 & 0.2 & 91.482 & 0.311 & 1 & 0.2 & 0.456 & Hartkopf \& Mason 2011b & \\
\hline $20099+2055$ & STF & $2637 \mathrm{BC}$ & 2016.814 & 215.0 & 0.2 & 95.916 & 0.326 & 1 & -0.3 & 0.439 & Hartkopf \& Mason 2011b & \\
\hline $20129+0959$ & HJ & 908 & 2016.814 & 350.3 & 0.2 & 32.528 & 0.111 & 1 & 0.4 & -0.461 & Hartkopf \& Mason 2011b & B \\
\hline $20213+0250$ & HLD & 158 & 2016.875 & 42.3 & 0.2 & 1.171 & 0.004 & 1 & -4.1 & -0.068 & Heintz 1998 & \\
\hline & & & & & & & & & 0.3 & -0.026 & Table 6 & \\
\hline $20312+0513$ & $\mathrm{AG}$ & 257 & 2016.645 & 74.9 & 0.2 & 1.659 & 0.006 & 1 & 0.3 & 0.046 & Zasche et al. 2009 & A \\
\hline $20352+0608$ & HWE & 98 & 2016.645 & 2.1 & 0.2 & 58.516 & 0.199 & 1 & -0.3 & 0.397 & Hartkopf \& Mason 2011b & \\
\hline $20387+3838$ & STF & $2708 \mathrm{AB}$ & 2016.853 & 323.3 & 0.2 & 57.444 & 0.195 & 1 & 0.5 & $\begin{array}{l}0.591 \\
0.131\end{array}$ & Hartkopf \& Mason 2011b & \\
\hline $20450+1244$ & $\mathrm{BU}$ & $64 \mathrm{AB}$ & 2002.602 & 350.0 & 0.2 & 0.678 & 0.002 & 3 & -1.0 & 0.018 & Brendley \& Hartkopf 2007 & $\mathrm{~A}, \mathrm{C}$ \\
\hline $20462+1554$ & STF & $2725 \mathrm{AB}$ & 2016.727 & 11.4 & 0.2 & 6.189 & 0.021 & 1 & -0.3 & 0.042 & Mason \& Hartkopf 2014 & \\
\hline $20467+1607$ & $\mathrm{STF}$ & $2727 \mathrm{AB}$ & 2005.485 & 265.9 & 0.2 & 9.061 & 0.031 & 1 & 0.3 & -0.100 & Hale 1994 & $\mathrm{~A}, \mathrm{C}$ \\
\hline $20467+1607$ & STF & $2727 \mathrm{AB}$ & 2016.727 & 265.6 & 0.2 & 8.966 & 0.030 & 1 & 0.6 & 0.025 & Hale 1994 & \\
\hline $20520+4346$ & STT & $416 \mathrm{AB}$ & 2016.853 & 118.1 & 0.2 & 9.606 & 0.033 & 1 & 0.5 & 0.112 & Hartkopf \& Mason 2011b & B \\
\hline $21003+6130$ & HJ & $1607 \mathrm{AB}$ & 2016.700 & 72.8 & 0.2 & 15.729 & 0.053 & 1 & -0.3 & 0.073 & Hartkopf \& Mason 2011b & \\
\hline $21031+0132$ & STF & $2744 \mathrm{AB}$ & 2016.716 & 109. & 0.2 & 1.227 & 0.009 & 2 & 6.6 & 0.034 & Popovic 1969 & A \\
\hline $21069+3845$ & STF & $2758 \mathrm{AB}$ & 2016.664 & 152.7 & 0.2 & 31.855 & 0.108 & 1 & 0.7 & 0.205 & Gorshanov et al. 2006 & \\
\hline $21144+2905$ & STF & $2779 \mathrm{AB}$ & 2016.659 & 164.1 & 0.2 & 15.212 & 0.052 & 1 & 0.0 & 0.064 & Hartkopf \& Mason 2011b & \\
\hline $21199+5841$ & HLM & 39 & 2016.661 & 63.3 & 0.2 & 18.771 & 0.064 & 1 & 0.5 & -0.014 & Hartkopf \& Mason 2011b & \\
\hline $21200+5259$ & STF & $2789 \mathrm{AB}$ & 2016.661 & 113.4 & 0.2 & 6.910 & 0.023 & 1 & -0.5 & 0.012 & Kiselev et al. 2009 & $\mathrm{E}$ \\
\hline $21208+3227$ & STT & $437 \mathrm{AB}$ & 2016.659 & 19.3 & 0.2 & 2.481 & 0.008 & 1 & 0.8 & 0.042 & Hartkopf \& Mason 2011a & $\mathrm{A}$ \\
\hline
\end{tabular}


Table 5-Continued

\begin{tabular}{|c|c|c|c|c|c|c|c|c|c|c|c|c|}
\hline $\begin{array}{l}\text { WDS Desig. } \\
\alpha, \delta(2000)\end{array}$ & $\begin{array}{l}\text { Dis } \\
\text { Des }\end{array}$ & $\begin{array}{l}\text { iscoverer } \\
\text { signation }\end{array}$ & JY & $\begin{array}{c}\theta \\
(0)\end{array}$ & $\begin{array}{l}\sigma \theta \\
(0)\end{array}$ & $\begin{array}{c}\rho \\
\left({ }^{\prime \prime}\right)\end{array}$ & $\begin{array}{l}\sigma \rho \\
\left({ }^{\prime \prime}\right)\end{array}$ & $\mathrm{n}$ & $\begin{array}{l}\mathrm{O}-\mathrm{C}_{\theta} \\
(0)\end{array}$ & $\begin{array}{c}\mathrm{O}-\mathrm{C}_{\rho} \\
\left({ }^{\prime \prime}\right)\end{array}$ & Reference & Note \\
\hline $21264-2025$ & HWE & $56 \mathrm{AC}$ & 2016.727 & 160.6 & 0.2 & 5.074 & 0.017 & 1 & 0.4 & 0.004 & Hartkopf \& Mason 2011b & \\
\hline $21289+1105$ & STF & $2799 \mathrm{AB}$ & 2016.653 & 259.4 & 0.2 & 1.911 & 0.006 & 1 & 1.1 & 0.033 & Hartkopf \& Mason 2011a & A \\
\hline $21328+5839$ & $\mathrm{HJ}$ & $1659 \mathrm{AB}$ & 2016.785 & 287.3 & 0.2 & 7.808 & 0.027 & 1 & -0.1 & -0.016 & Hartkopf \& Mason 2011b & \\
\hline $21330+2043$ & $\mathrm{STF}$ & $2804 \mathrm{AB}$ & 2016.653 & 358.5 & 0.2 & 3.375 & 0.011 & 1 & 0.0 & 0.034 & Hartkopf \& Mason 2011b & B \\
\hline $21370+8255$ & $\mathrm{STF}$ & 2837 & 2016.700 & 269.2 & 0.2 & 3.205 & 0.011 & 1 & 0.9 & 0.119 & Kiselev et al. 2009 & A \\
\hline $21520+5548$ & STF & $2840 \mathrm{AB}$ & 2016.683 & 196.1 & 0.2 & 17.789 & 0.060 & 1 & 0.3 & 0.079 & Hartkopf \& Mason 2011b & \\
\hline $21555+5232$ & $\mathrm{STT}$ & $456 \mathrm{AC}$ & 2016.683 & 292.1 & 0.2 & 26.140 & 0.089 & 1 & 1.1 & -0.171 & Hartkopf \& Mason 2011b & \\
\hline $21582+8252$ & STF & $2873 \mathrm{AB}$ & 2016.700 & 65.9 & 0.2 & 13.960 & 0.047 & 1 & $\begin{array}{r}-0.1 \\
0.1\end{array}$ & $\begin{array}{l}0.218 \\
0.221\end{array}$ & $\begin{array}{l}\text { Grosheva } 2006 \\
\text { Grosheva } 2006\end{array}$ & \\
\hline $21584+5245$ & ES & $1015 \mathrm{AB}$ & 2016.683 & 239.4 & 0.2 & 8.197 & 0.028 & 1 & 0.5 & 0.051 & Hartkopf \& Mason 2011b & \\
\hline $22033+6051$ & $\mathrm{STF}$ & $2860 \mathrm{AB}$ & 2016.700 & 256.9 & 0.2 & 13.420 & 0.046 & 1 & 0.1 & 0.234 & Hartkopf \& Mason 2011b & B \\
\hline $22038+6438$ & STF & $2863 \mathrm{AB}$ & 2005.720 & 274.9 & 0.2 & 8.005 & 0.027 & 1 & 0.6 & -0.284 & Zeller 1965 & $\mathrm{~A}, \mathrm{C}$ \\
\hline $22038+6438$ & $\mathrm{STF}$ & $2863 \mathrm{AB}$ & 2016.703 & 274.8 & 0.2 & 8.120 & 0.028 & 1 & 1.1 & -0.297 & Zeller 1965 & \\
\hline $22057+2954$ & HJ & 1721 & 2016.815 & 264.7 & 0.2 & 12.759 & 0.043 & 1 & -0.2 & -0.007 & Hartkopf \& Mason 2011b & \\
\hline $22086+5917$ & $\mathrm{STF}$ & $2872 \mathrm{BC}$ & 2016.703 & 296.2 & 0.2 & 0.777 & 0.003 & 1 & -0.6 & -0.025 & Seymour et al. 2002 & A \\
\hline $22143+1711$ & $\mathrm{STF}$ & $2877 \mathrm{AB}$ & 2016.662 & 24.2 & 0.2 & 23.935 & 0.081 & 1 & 0.5 & 0.372 & Hartkopf \& Mason 2011b & B \\
\hline $22207+2457$ & STF & $2895 \mathrm{AB}$ & 2016.757 & 48.3 & 0.2 & 14.001 & 0.048 & 1 & 0.3 & 0.094 & Hartkopf \& Mason 2011b & \\
\hline $22226+3328$ & $\mathrm{ES}$ & 390 & 2016.760 & 268.1 & 0.2 & 8.409 & 0.029 & 1 & -0.4 & 0.145 & Hartkopf \& Mason 2011b & B \\
\hline $22241-0450$ & $\mathrm{BU}$ & $172 \mathrm{AB}$ & 2016.727 & 30.9 & 0.2 & 0.461 & 0.002 & 1 & 1.2 & -0.011 & Tokovinin et al. 2015 & A \\
\hline $22266-1645$ & SHJ & $345 \mathrm{AB}$ & 2016.727 & 71.9 & 0.2 & 1.206 & 0.004 & 1 & 2.6 & -0.078 & Hale 1994 & A \\
\hline $22288-0001$ & STF & 2909 АВ & 2016.727 & 162.6 & 0.2 & 2.343 & 0.008 & 1 & 3.8 & 0.136 & Tokovinin 2016 & A \\
\hline $22326+0725$ & $\mathrm{STF}$ & 2915 AB & 2016.739 & 125.5 & 0.6 & 15.261 & 0.023 & 2 & 0.6 & 0.079 & Hartkopf \& Mason 2011b & \\
\hline $22361+7253$ & $\mathrm{BU}$ & 1092 АВ & 2016.700 & 222.6 & 0.2 & 0.358 & 0.001 & 1 & 2.9 & -0.005 & Docobo \& Campo 2016 & A \\
\hline $22396-1237$ & $\mathrm{STF}$ & 2928 AB & 2016.757 & 280.6 & 0.2 & 3.076 & 0.010 & 1 & -0.6 & -0.118 & Hartkopf \& Mason 2011b & B \\
\hline $22419+2126$ & $\mathrm{STF}$ & 2934 & 2016.910 & 56.5 & 0.2 & 1.401 & 0.005 & 3 & 2.8 & 0.040 & Zirm 2013 & A \\
\hline $22460+1915$ & $\mathrm{STF}$ & 2941 & 2016.880 & 258.2 & 0.3 & 14.765 & 0.009 & 2 & 0.5 & 0.039 & Hartkopf \& Mason 2011b & \\
\hline $22478-0414$ & STF & $2944 \mathrm{AB}$ & 2016.757 & 305.2 & 0.2 & 1.833 & 0.006 & 1 & 0.2 & 0.014 & Zirm 2007 & \\
\hline $22478-0414$ & STF & $2944 \mathrm{AC}$ & 2016.757 & 85.8 & 0.2 & 62.183 & 0.211 & 1 & 0.6 & 0.928 & Hartkopf \& Mason 2011b & B \\
\hline $22490+6834$ & STF & 2947 AB & 2016.850 & 55.7 & 0.2 & 4.681 & 0.016 & 1 & 0.6 & -0.013 & Hartkopf \& Mason 2011b & \\
\hline $22557+1547$ & $\mathrm{HU}$ & 987 & 2016.911 & 76.2 & 0.1 & 1.196 & 0.004 & 3 & -0.5 & 0.046 & Brendley \& Hartkopf 2007 & A \\
\hline $23077+0636$ & $\mathrm{STF}$ & $2976 \mathrm{AC}$ & 2016.956 & 209.4 & 0.3 & 21.373 & 0.033 & 2 & 1.0 & 0.148 & Hartkopf \& Mason 2011b & \\
\hline $23114+3813$ & $\mathrm{HO}$ & $197 \mathrm{AB}, \mathrm{C}$ & 2016.817 & 319.4 & 0.2 & 36.007 & 0.122 & 1 & 0.1 & 0.230 & Hartkopf \& Mason 2011b & B \\
\hline $23114+3813$ & $\mathrm{HO}$ & $197 \mathrm{AB}, \mathrm{D}$ & 2016.817 & 278.9 & 0.2 & 57.025 & 0.194 & 1 & 0.2 & 0.330 & Hartkopf \& Mason 2011b & B \\
\hline $23133+2205$ & STF & $2990 \mathrm{AB}$ & 2016.760 & 56.0 & 0.2 & 2.598 & 0.009 & 1 & 0.6 & 0.042 & Hartkopf \& Mason 2011b & B \\
\hline $23186+6807$ & $\mathrm{STF}$ & $3001 \mathrm{AB}$ & 2002.559 & 220.7 & 0.2 & 3.489 & 0.012 & 1 & 0.3 & 0.187 & Docobo et al. 2003 & A \\
\hline $23212+3526$ & $\mathrm{STF}$ & $3006 \mathrm{AB}$ & 2016.839 & 151.9 & 0.2 & 7.341 & 0.025 & 1 & 0.7 & 0.011 & Hartkopf \& Mason 2011b & \\
\hline $23238-0828$ & $\mathrm{STF}$ & 3008 & 2016.815 & 147.7 & 0.2 & 6.984 & 0.024 & 1 & 0.2 & 0.092 & Hartkopf \& Mason 2011b & B \\
\hline $23317+1956$ & WIR & $1 \mathrm{AB}$ & 2004.923 & 86.9 & 0.2 & 5.285 & 0.018 & 2 & -1.3 & -0.148 & Heintz 1984 & A \\
\hline $23431+1150$ & A & 1242 & 2016.826 & 339.6 & 0.2 & 1.056 & 0.004 & 1 & -0.1 & 0.069 & Ling 2004 & \\
\hline $23487+6453$ & $\mathrm{STT}$ & $507 \mathrm{AB}$ & 2016.785 & 322.3 & 0.2 & 0.731 & 0.002 & 1 & 3.7 & 0.023 & Zulevic 1977 & A \\
\hline $23536+5131$ & $\mathrm{STT}$ & A251 AB & 2016.785 & 208.3 & 0.2 & 48.242 & 0.164 & 1 & 0.5 & 0.319 & Hartkopf \& Mason 2011b & \\
\hline $23564-0930$ & $\mathrm{STF}$ & $3046 \mathrm{AB}$ & 2016.818 & 269.1 & 0.2 & 3.953 & 0.013 & 1 & 0.8 & -0.038 & Hartkopf \& Mason 2011b & \\
\hline $23568+0444$ & A & 2100 & 2002.854 & 277.3 & 0.2 & 0.328 & 0.001 & 1 & 1.1 & 0.051 & Mason \& Hartkopf 2012 & \\
\hline $23595+3343$ & $\mathrm{STF}$ & $3050 \mathrm{AB}$ & 2016.818 & 340.6 & 0.2 & 2.465 & 0.008 & 1 & -0.0 & 0.055 & Hartkopf \& Mason 2011a & A \\
\hline
\end{tabular}

A : Residuals indicate this system should be monitored for possible orbit improvement.

B : Residuals indicate this linear solution may be ready for update soon.

C : This would have been published in an earlier paper in this series, but given the large change in position this measure was not published until its confirmation.

D : Two solutions in Kiselev et al. (2009). The one whose residuals are given here $\left(\Omega=242^{\circ}\right)$ is definitely better.

E : Two solutions in Kiselev et al. (2009). The one whose residuals are given here $\left(\Omega=290^{\circ}\right)$ is definitely better. 
Table 6. Improved Orbital Elements

\begin{tabular}{|c|c|c|c|c|c|c|c|c|c|c|c|}
\hline $\begin{array}{c}\text { WDS } \\
\text { Designation }\end{array}$ & $\begin{array}{l}\mathrm{Di} \\
\mathrm{De}\end{array}$ & $\begin{array}{l}\text { iscoverer } \\
\text { signation }\end{array}$ & $\begin{array}{c}\mathrm{P} \\
(\mathrm{yr})\end{array}$ & $\begin{array}{c}\mathrm{a} \\
\left({ }^{\prime \prime}\right)\end{array}$ & $\begin{array}{l}\mathrm{i} \\
\left({ }^{\circ}\right)\end{array}$ & $\begin{array}{l}\Omega \\
\left({ }^{\circ}\right)\end{array}$ & $\begin{array}{l}\mathrm{T}_{0} \\
(\mathrm{yr})\end{array}$ & e & $\begin{array}{l}\omega \\
\left({ }^{\circ}\right)\end{array}$ & Reference & $\mathrm{Gr}$ \\
\hline \multicolumn{12}{|c|}{ Improved but still Provisional Orbital Elements } \\
\hline $00162+7657$ & $\mathrm{STF}$ & 13 & 1245.6 & 1.078 & 140.7 & 86.1 & 3048.6 & 0.472 & 287.6 & Olevic \& Jovanovic 2001 & 4 \\
\hline \multicolumn{12}{|c|}{ Reliable Orbital Elements } \\
\hline $08269+3212$ & $\mathrm{HU}$ & $714 \mathrm{Ba}, \mathrm{Bb}$ & $\begin{array}{r}195.5 \\
\pm 13.2\end{array}$ & $\begin{array}{r}0.535 \\
\pm 0.015\end{array}$ & $\begin{array}{r}72.4 \\
\pm 3.3\end{array}$ & $\begin{array}{r}135.9 \\
\pm 1.6\end{array}$ & $\begin{array}{r}1943.2 \\
\pm 2.5\end{array}$ & $\begin{array}{r}0.567 \\
\pm 0.040\end{array}$ & $\begin{array}{r}3.7 \\
\pm 6.7\end{array}$ & Baize 1993 & 4 \\
\hline $19282-1209$ & SCJ & 22 & $\begin{array}{r}170.2 \\
\pm 1.3\end{array}$ & $\begin{array}{r}1.0031 \\
\pm 0.0059\end{array}$ & $\begin{array}{r}10.4 \\
\pm 3.0\end{array}$ & $\begin{array}{c}206 \\
\pm 18\end{array}$ & $\begin{array}{r}1983.91 \\
\pm 0.15\end{array}$ & $\begin{array}{r}0.5837 \\
\pm 0.0038\end{array}$ & $\begin{array}{c}308 \\
\pm 17\end{array}$ & Docobo \& Ling 2009 & 3 \\
\hline $20213+0250$ & HLD & 158 & $\begin{array}{r}232.4 \\
\pm 7.8\end{array}$ & $\begin{array}{r}0.858 \\
\pm 0.017\end{array}$ & $\begin{array}{r}128.6 \\
\pm 2.1\end{array}$ & $\begin{array}{r}218.3 \\
\pm 1.9\end{array}$ & $\begin{array}{r}1961.16 \\
\pm 0.52\end{array}$ & $\begin{array}{r}0.746 \\
\pm 0.012\end{array}$ & $\begin{array}{r}17.7 \\
\pm 2.6\end{array}$ & Heintz 1998 & 4 \\
\hline
\end{tabular}

Table 7. Ephemerides for New Orbit Solutions

\begin{tabular}{|c|c|c|c|c|c|c|c|c|c|c|c|}
\hline \multirow{2}{*}{$\begin{array}{c}\text { WDS } \\
\text { Designation }\end{array}$} & \multirow{2}{*}{$\begin{array}{l}\text { Discoverer } \\
\text { Designation }\end{array}$} & \multicolumn{2}{|c|}{2018.0} & \multicolumn{2}{|c|}{2020.0} & \multicolumn{2}{|c|}{2022.0} & \multicolumn{2}{|c|}{2024.0} & \multicolumn{2}{|c|}{2026.0} \\
\hline & & $\begin{array}{c}\theta \\
(0)\end{array}$ & $\begin{array}{c}\rho \\
\left({ }^{\prime \prime}\right)\end{array}$ & $\begin{array}{c}\theta \\
(\circ)\end{array}$ & $\begin{array}{c}\rho \\
\left({ }^{\prime \prime}\right)\end{array}$ & $\begin{array}{c}\theta \\
(\circ)\end{array}$ & $\begin{array}{c}\rho \\
\left({ }^{\prime \prime}\right)\end{array}$ & $\begin{array}{c}\theta \\
(\circ)\end{array}$ & $\begin{array}{c}\rho \\
\left({ }^{\prime \prime}\right)\end{array}$ & $\begin{array}{c}\theta \\
(0)\end{array}$ & $\begin{array}{c}\rho \\
\left({ }^{\prime \prime}\right)\end{array}$ \\
\hline $00162+7657$ & STF 13 & 48.3 & 0.957 & 47.8 & 0.960 & 47.3 & 0.962 & 46.8 & 0.964 & 46.3 & 0.967 \\
\hline $08269+3212$ & $\mathrm{HU} \quad 714 \mathrm{Ba}, \mathrm{Bb}$ & 312.6 & 0.792 & 313.0 & 0.801 & 313.4 & 0.808 & 313.8 & 0.815 & 314.2 & 0.821 \\
\hline $19282-1209$ & SCJ $\quad 22$ & 290.9 & 1.131 & 293.5 & 1.166 & 296.0 & 1.199 & 298.3 & 1.231 & 300.4 & 1.261 \\
\hline $20213+0250$ & HLD 158 & 41.7 & 1.209 & 41.1 & 1.230 & 40.4 & 1.250 & 39.8 & 1.269 & 39.3 & 1.287 \\
\hline
\end{tabular}

Table 8. Double Stars Not Found

\begin{tabular}{|c|c|c|c|c|c|c|c|c|c|}
\hline \multirow{2}{*}{$\begin{array}{c}\text { WDS } \\
\text { Designation }\end{array}$} & \multirow{2}{*}{\multicolumn{2}{|c|}{$\begin{array}{l}\text { Discoverer } \\
\text { Designation }\end{array}$}} & \multirow{2}{*}{$\begin{array}{l}\text { Most } \\
\text { Date }\end{array}$} & \multirow{2}{*}{$\begin{array}{c}\text { Recent } \\
\theta \\
(\mathrm{o})\end{array}$} & \multicolumn{2}{|c|}{ Published Observation } & \multicolumn{2}{|c|}{ Published Magnitudes } & \multirow[t]{2}{*}{ Notes } \\
\hline & & & & & $\begin{array}{c}\rho \\
\left({ }^{\prime \prime}\right)\end{array}$ & Reference & Primary & Secondary & \\
\hline $03472+2522$ & POU & 310 & 1894 & 100 & 16.0 & Pourteau 1933 & 11.4 & 11.4 & \\
\hline $07078-0822$ & OL & 168 & 1935 & 157 & 4.09 & Olivier 1939 & 10.0 & 10.4 & \\
\hline $08419+3546$ & SEI & 508 & 1895 & 61 & 25.70 & Scheiner 1908 & 11.0 & 11.0 & 1 \\
\hline $23194+2417$ & $\mathrm{POU}$ & 5798 & 1894 & 90 & 19.1 & Pourteau 1933 & 11.8 & 11.8 & \\
\hline
\end{tabular}

1: Neither component seen on POSS plate; may be flaws on AC Potsdam plate. Also unresolved in Berko (2009). 